Mahidol Oxford Tropical Medicine Research Unit, Affiliated Research Centre of The Open University

\title{
The Economics of Vivax Malaria Treatment
}

\author{
Angela Devine BSc, MSc
}

Thesis offered for the degree of Doctor of Philosophy

The Open University, UK

23 December 2017

Health Economics 



\begin{abstract}
The control and eventual elimination of malaria will require widescale adoption of strategies to ensure early diagnosis and highly effective treatment of infected individuals. In Plasmodium vivax, multiple relapse episodes can only be averted by treating the dormant liver stage of the parasite life cycle - a strategy known as radical cure. This thesis aims to investigate key factors determining treatment-seeking behaviour and costs associated with vivax malaria, how these influence healthcare decisions, the cost-effectiveness of screening tests and treatments for radical cure, and the cost-benefit implications of their global implementation.

Treatment-seeking behaviour, assessed in Papua, Indonesia, demonstrated that household costs per person seeking treatment for vivax malaria were similar to those for falciparum malaria. Switching from ineffective to effective malaria treatment in the public sector improved the behaviour of patients and both public and private healthcare providers. Diagnostic strategies were investigated on the Thai-Myanmar border, where screening for glucose-6phosphate-dehydrogenase (G6PD) deficiency prior to radical cure reduced the disease burden while being a cost-effective option for healthcare providers that do not use radical cure and a potentially cost-saving alternative where primaquine is prescribed without screening. The costs of implementing G6PD screening were collected in three countries, and highlighted that RDTs consistently reduced costs as compared to the widely-used fluorescent spot test. Across four countries, the indirect cost due to lost productivity was the largest cost component for households of patients with vivax malaria. After combining provider and patient costs with case estimates, the global economic burden of vivax malaria was estimated to be US $\$ 330$ million per year. Adopting a policy of screening for G6PD deficiency with delivery of highly effective radical cure was projected to save US $\$ 45$ million.
\end{abstract}

P. vivax malaria causes a large economic burden that can be reduced substantially by delivering safe and effective radical cure. 


\section{The Candidate's Contribution \& Associated Publications}

The candidate was the primary contributor to all material presented in this thesis as outlined below alongside the contributions by supervisors and co-authors.

- Chapter 1: The candidate was the primary author with review from $\mathrm{PhD}$ supervisors. The candidate conducted the literature review.

- Chapter 2: The candidate was the primary author with review from PhD supervisors and co-authors. The candidate was responsible for merging and cleaning the data, creating a data dictionary and analysing the data. The analysis plan was developed with assistance from the PhD supervisors. The candidate was not involved in the survey design or execution. This has been published as Karyana M, Devine A (joint first author), Kenangalem E, Burdarm L, Poespoprodjo JR, Vemuri R, Anstey NM, Tjitra E, Price RN, Yeung S. Treatment-seeking behaviour and associated costs for malaria in Papua, Indonesia. Malar J. 2016;15(1):536. https://doi.org/10.1186/s12936-016-1588-8. PMID: 27821127.

- Chapter 3: The candidate was the primary author with review from PhD supervisors and co-authors. The candidate was responsible for merging and cleaning the data, creating a data dictionary and analysing the data. The candidate was not involved in the survey design or execution. This has been published as Devine A, Kenangalem E, Burdarm FH, Anstey NM, Poespoprodjo JR, Price RN, Yeung S. Treatment-seeking behavior after the implementation of a unified policy of dihydroartemisin-piperaquine for the treatment of uncomplicated malaria in Papua, Indonesia. Am J Trop Med Hyg. 2018;98(2):543550. https: //doi.org/10.4269/ajtmh.17-0680. PMID: 29280424. 
- Chapter 4: The candidate was the primary author with review from PhD supervisors and co-authors. The candidate wrote the model code, selected the model parameters, designed and developed an interactive web-based model application (see https://malaria.shinyapps.io/g6pd_screening/) and analysed the model results. This has been published as Devine A, Parmiter M, Chu CS, Bancone G, Nosten F, Price RN, Lubell Y, Yeung S. Using G6PD tests to enable the safe treatment of Plasmodium vivax infections with primaquine on the Thailand-Myanmar border: A cost-effectiveness analysis. PLoS Negl Trop Dis. 2017;11(5):e0005602. https://doi.org/10.1371/journal.pntd.0005602 PMID: 28542194.

- Chapter 5: The candidate was the primary author with review from PhD supervisors. The candidate designed the facility surveys, oversaw the data collection, and analysed the data.

- Chapter 6: The candidate was the primary author with review from PhD supervisors. The candidate reviewed the literature for data sources, designed the model structure and analysed the data.

- Chapter 7: Primary author with review from PhD supervisors. 


\section{Acknowledgements}

I could probably write another thesis about the individuals who helped me to write this one. Instead, I have opted for the short version. The first big thank you goes to my supervisors for giving me this opportunity, providing me with guidance, and instilling a sense of enthusiasm for a disease that I once knew nothing about. I am also deeply thankful to the colleagues and friends who cheered me on and kept me sane. To Oh, for being my $\mathrm{PhD}$ comrade. To the MAEMOD team, for being such fun and thoughtful workmates. And to Kate and Bethany, for walking with me through the most difficult times.

This thesis is dedicated to my mother, whose untold strength has always been an inspiration. 


\section{Contents}

Abstract

Authorship $\quad$ v

Acknowledgements $\quad$ vii

1 Introduction 1

1.1 Epidemiology . . . . . . . . . . . . . . . . . . 3

1.2 Vivax malaria treatment . . . . . . . . . . . . . . . 5

1.3 The challenge of G6PD deficiency . . . . . . . . . . . . . 6

1.4 Screening for G6PD deficiency and future options for radical cure . . . 8

1.5 Prospects and challenges for inclusion of $P$. vivax in malaria elimination campaigns . . . . . . . . . . . . . . . . . . . . 9 9

1.6 The intersection of $P$. vivax with health economics . . . . . . . . . . 10

1.7 The economics of $P$. vivax treatment . . . . . . . . . . . . . . . 13

1.8 Aims and objectives . . . . . . . . . . . . . . . . . . 14

References for Chapter $1 \ldots \ldots \ldots \ldots$

2 Treatment-seeking behaviour and associated costs for malaria in Papua, Indonesia $\quad 27$

2.1 Introduction . . . . . . . . . . . . . . . . . . . 27

2.2 Methods . . . . . . . . . . . . . . . . . . . . 29

2.2.1 Site details . . . . . . . . . . . . . . . . . . . . . . . 29

2.2.2 Household sampling strategy . . . . . . . . . . . . . . . 30

2.2 .3 Data collection . . . . . . . . . . . . . . . . . 31

2.2.4 Data analysis . . . . . . . . . . . . . . . . . 32

2.3 Results . . . . . . . . . . . . . . . . . . . . . . . . 34

2.3.1 Household demographics, SES and expenditure . . . . . . . 34

2.3.2 History of fevers and parasite positivity of household members . 38

2.3.3 Treatment-seeking behaviour of household members . . . . . . . 40

2.3.4 Diagnosis of malaria at healthcare providers . . . . . . . . . . . 40

2.3.5 Antimalarial treatment at healthcare providers . . . . . . . . . . 41

2.3.6 Direct costs of treatment seeking . . . . . . . . . . . . . . 43

2.3.7 Indirect costs per fever episode . . . . . . . . . . . . . . . 43

2.3.8 Malaria costs from healthcare facility exit survey . . . . . . . . 45

2.4 Discussion . . . . . . . . . . . . . . . . . . 47

2.4 Conclusions . . . . . . . . . . . . . . . . . 51

2.5 Declarations . . . . . . . . . . . . . . . . 52

2.5.1 Acknowledgements . . . . . . . . . . . . . 52 
2.5.2 Ethics approval . . . . . . . . . . . . . . . . . 52

References for Chapter $2 \ldots \ldots \ldots$. . . . . . . . . . . . . 53

3 Treatment seeking after switch to unified malaria treatment in Papua 57

3.1 Introduction . . . . . . . . . . . . . . . . 58

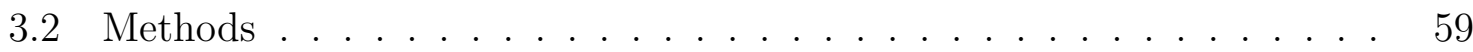

3.2.1 Data collection . . . . . . . . . . . . . . 61

3.2 .2 Data analysis . . . . . . . . . . . . . . . 6 61

3.3 Results . . . . . . . . . . . . . . . . . . 62

3.3.1 Household characteristics . . . . . . . . . . . . . . 62

3.3.2 History of fever and parasite positivity of household members . 65

3.3.3 Treatment-seeking behaviour . . . . . . . . . . . . . . 66

3.3.4 Diagnosis of malaria at healthcare providers . . . . . . . . . . 66

3.3.5 Antimalarial treatment at healthcare providers . . . . . . . . . 70

3.3.6 Cost burden of fever . . . . . . . . . . . . . . . . . 72

3.4 Discussion . . . . . . . . . . . . . . . . . . . . . . . 73

3.5 Declarations . . . . . . . . . . . . . . . . . 77

3.5.1 Acknowledgments . . . . . . . . . . . . . . 77

3.5.2 Ethics approval . . . . . . . . . . . . . . . . . . . . . . 77

References for Chapter $3 \ldots \ldots \ldots$. . . . . . . . . . . . 78

4 Cost-effectiveness of using G6PD tests before primaquine treatment $\begin{array}{ll}\text { for } P \text {. vivax } & 81\end{array}$

4.1 Introduction . . . . . . . . . . . . . . . . . . . 81

4.2 Methods . . . . . . . . . . . . . . . . . . . . 83

4.2.1 Base case analysis . . . . . . . . . . . . . . . . 83

4.2 .2 Sensitivity analyses . . . . . . . . . . . . . . . . . . . . . . . . . . . . . . 90

4.3 Results . . . . . . . . . . . . . . . . . . . . . 91

4.3.1 Comparison with the chloroquine strategy . . . . . . . . . . 91

4.3.2 Comparison with the primaquine strategy . . . . . . . . . . 93

4.3.3 Web-based application . . . . . . . . . . . . . . . 97

4.4 Discussion . . . . . . . . . . . . . . . . . . . . . . . 97

4.5 Appendix: Study description for determining G6PD activity in females 104

4.5.1 Study population and samples . . . . . . . . . . . . . . . . . 104

4.5.2 Laboratory methods . . . . . . . . . . . . . . . . . . . . . . . 104

4.5.3 Statistical analysis . . . . . . . . . . . . . . . . . . 104

4.5.4 Results . . . . . . . . . . . . . . . . . . . . 104

4.6 Declarations . . . . . . . . . . . . . . . . . . . . 105

4.6.1 Acknowledgments . . . . . . . . . . . . . . . . 105

4.6 .2 Funding . . . . . . . . . . . . . . . . . . . . . . . . . . . . . . . . . . . . . .

References for Chapter $4 \ldots \ldots \ldots \ldots$

5 Economic costs of $P$. vivax episodes: A multi-country comparative $\begin{array}{lr}\text { analysis using primary trial data } & 113\end{array}$

5.1 Introduction . . . . . . . . . . . . . . . . . . 113

5.2 Methods . . . . . . . . . . . . . . . . . . . 115

5.2 .1 Clinical trial details . . . . . . . . . . . . . . . . 115

5.2 .2 Household cost collection . . . . . . . . . . . . . . . 116

5.2.3 Provider cost data collection . . . . . . . . . . . . . . 116 
5.2 .4 Analysis . . . . . . . . . . . . . . . . . . 118

5.3 Results . . . . . . . . . . . . . . . . . . . . . . . . . . . . 118

5.3.1 Patient characteristics . . . . . . . . . . . . . . . 118

5.3.2 Indirect household costs and usual activities . . . . . . . . . . . 119

5.3.3 Treatment-seeking behaviour and associated costs . . . . . . . 120

5.3.4 Direct and indirect costs to patients from the enrolment up to the follow up visit . . . . . . . . . . . . . . . . 122

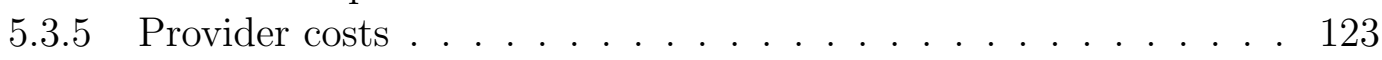

5.4 Discussion . . . . . . . . . . . . . . . . . . . . 126

5.5 Appendix: Tables in international dollars . . . . . . . . . . . . . 131

5.6 Declarations . . . . . . . . . . . . . . . . . 135

5.6 .1 Acknowledgments . . . . . . . . . . . . . . . 135

5.6 .2 Ethics approval . . . . . . . . . . . . . . . . . 135

References for Chapter $5 \ldots \ldots \ldots \ldots$. . . . . . . . . . 136

6 Global economic costs due to vivax malaria treatment and the potential cost-benefit of radical cure 141

6.1 Introduction . . . . . . . . . . . . . . . . . . . . . . . 141

6.2 Methods . . . . . . . . . . . . . . . . . . . 143

6.2 .1 Summary . . . . . . . . . . . . . . . . . . 143

6.2 .2 Cases of vivax malaria . . . . . . . . . . . . . . . . . 145

6.2 .3 Usual care for vivax malaria . . . . . . . . . . . . . . . . . . 145

6.2 .4 Cost data . . . . . . . . . . . . . . . . . . 146

6.2.5 Costs of productivity losses . . . . . . . . . . . . . . . . . 148

6.2.6 Low and high cost bounds . . . . . . . . . . . . . . . . . 149

6.2.7 Global cost-benefit of implementing G6PD testing with highly effective radical cure . . . . . . . . . . . . . . . . . 150

6.2.8 Global cost-benefit of radical cure with low adherence . . . . . . 152

6.3 Results . . . . . . . . . . . . . . . . . . . . 153

6.3.1 Global cost burden estimates . . . . . . . . . . . . 153

6.3.2 Global cost-benefit of implementing effective G6PD testing and radical cure . . . . . . . . . . . . . . . . 153

6.3.3 Global cost-benefit of radical cure with low adherence . . . . . . 156

6.4 Discussion . . . . . . . . . . . . . . . . . 156

6.5 Declarations . . . . . . . . . . . . . . . . 166

6.5.1 Acknowledgments . . . . . . . . . . . . . 166

References for Chapter $6 \ldots \ldots \ldots$. . . . . . . . . . . 167

7 Discussion $\quad \mathbf{1 7 3}$

7.1 Summary . . . . . . . . . . . . . . . . . . . . 173

7.2 Empirical findings . . . . . . . . . . . . . . . . . . . . . . . 174

7.3 Methodological approaches . . . . . . . . . . . . . . . . 177

7.4 Policy implications . . . . . . . . . . . . . . . . . . . . . . . . . . . . . . . . . . . . . . . . . . . . . . . .

7.5 Limitations . . . . . . . . . . . . . . . . . . . . . . . . . 179

7.6 Future research directions . . . . . . . . . . . . . . . 183

7.6.1 Utilising the cost-effectiveness model . . . . . . . . . . . . . . 183

7.6.2 Targeting vulnerable risk groups . . . . . . . . . . . . . . . . . 184

7.6.3 Unified drug treatment . . . . . . . . . . . . . . . . . . . 185

7.7 Conclusion . . . . . . . . . . . . . . . . . . . . . . 187 
References for Chapter $7 \ldots \ldots \ldots \ldots$. . . . . . . . . . 188 


\section{List of Tables}

2.1 Surveys, questionnaires included in the surveys, sample sizes and dates 35

2.2 Demographic characteristics of individuals in surveyed households . . . 38

2.3 Logistic regression analysis of risk factors for reporting a fever in the past month . . . . . . . . . . . . . . . . . 39

2.4 Sociodemographic characteristics associated with whether ever sought treatment at a public provider . . . . . . . . . . . . . . 41

2.5 Percentage of respondents who reported receiving blood tests, antimalarials and associated costs . . . . . . . . . . . . . .

2.6 Antimalarial treatment received according to blood test results as reported in the household survey . . . . . . . . . . . . . . . 44

2.7 Reported direct, indirect and total costs per individual taking treatment for fever over the entire fever episode . . . . . . . . . . . . .

2.8 Mean, SD, median and IQR of patient costs per visit to a public provider by malaria diagnosis from facility exit surveys . . . . . . . . . . . 47

3.1 Policy recommendations for malaria . . . . . . . . . . . . . . . . 59

3.2 Demographic characteristics of all household members and individuals present during surveys in 2005 and 2013 . . . . . . . . . . . . . . 63

3.3 Fever reporting in those who were present during the survey in 2005 and

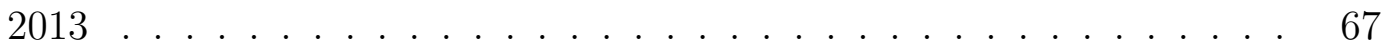

3.4 Multivariable analysis of risk factors for febrile illness in the past month 68

3.5 Changes in parasite prevalence by location . . . . . . . . . . . . 69

3.6 Percentage of respondents who reported receiving blood tests and antimalarials by survey . . . . . . . . . . . . . . . 71

3.7 Reported antimalarial treatment for individuals by reported diagnosis . $\quad 72$

3.8 Reported direct, indirect and total costs per individual taking treatment for fever over the entire fever episode . . . . . . . . . . . 73

4.1 Summary of key components of each strategy . . . . . . . . . . . . . 85

4.2 Model parameters and sources . . . . . . . . . . . . . . . . . 89

4.3 Costs and DALY results over one year per primary episode treated in the base case analysis and PSA . . . . . . . . . . . . . .

4.4 Simulated outcomes for 1,000 P. vivax malaria patients of undetermined G6PD status at attendance, if they were managed according to each of three strategies (chloroquine, primaquine and screening) . . . . . . .

5.1 Days unable to do usual activities and days a caretaker is required by country and associated indirect costs . . . . . . . . . . . . . . . . 120

5.2 Usual activities for patients and their carers . . . . . . . . . . . 120 
5.3 Direct, indirect and total household costs per patient with vivax malaria 122

5.4 Median costs and IQR compared by age and gender . . . . . . . . . . . 123

5.5 Cost of usual care for P. vivax infections . . . . . . . . . . . . . . . . 123

5.6 Breakdown of reported monthly overhead costs . . . . . . . . . . . . . 124

5.7 Cost per patient and time per test required for laboratory tests . . . . 125

5.8 Cost per FST and G6PD RDT administered . . . . . . . . . . . . . . 126

5.9 Direct, indirect and total household costs per patient with vivax malaria in I\$ . . . . . . . . . . . . . . . . . . . . . . . . 131

5.10 Median costs and IQR compared by age and gender in I\$ . . . . . . . 132

5.11 Cost of usual care for P. vivax infections in $I \$ \ldots . . . . . .132$

5.12 Breakdown of reported monthly overhead costs in I\$ . . . . . . . . . . 133

5.13 Cost per FST and G6PD RDT administered in I\$ . . . . . . . . . . . 133

5.14 Cost per patient and time per test required for laboratory tests in $\mathrm{I} \$$. 134

6.1 Key assumptions used in the base case global cost estimates . . . . . . 144

6.2 Case estimates, mean number of productive days lost by patients and their carers, diagnostic tests, treatment, ineligibility for primaquine and G6PD deficiency in those who are eligible for primaquine . . . . . . . . 147

6.3 Unit costs and assumptions by WHO region . . . . . . . . . . . . . . . 148

6.4 How household cost categories were applied by treatment-seeking behaviour 149

6.5 Countries and relapse incidence among cases per person year observed by zoogeographical zone . . . . . . . . . . . . . . . . . . 152

6.6 Base case global cost estimates by country . . . . . . . . . . . . . . 154

6.7 Results of the Highly effective radical cure scenario as compared to the base case estimates . . . . . . . . . . . . . . . . . . . . . 157

6.8 Results of the Low adherence to radical cure scenario as compared to the base case estimates . . . . . . . . . . . . . . . . . . . . 159 


\section{List of Figures}

1.1 The spatial distribution of $P$. vivax endemicity . . . . . . . . . . . . 2

1.2 The $P$. vivax life cycle . . . . . . . . . . . . . . . . . . . . 4

2.1 Flow diagram of household members, interviews, initial location of treatment taking, and whether took a second treatment in $2005 \ldots 36$

2.2 Results from the Discriminant Analysis of Principal Components to construct SES groupings for $2005 \ldots$. . . . . . . . . . . . 37

2.3 Mean household expenditure on health by SES groups . . . . . . . . . . 37

2.4 Histogram of the reported number of days of primaquine treatment for P. falciparum and P. vivax / mixed infections . . . . . . . . . . .

3.1 Flow diagram of household members, interviews, initial location of treatment taking, and whether took a second treatment in 2013. . . . . . .

3.2 Results from the Discriminant Analysis of Principal Components to construct SES groupings for 2013 . . . . . . . . . . . . 65

4.1 Model diagram of strategies for P. vivax treatment in males . . . . . . 86

4.2 Model diagram of strategies for $P$. vivax treatment in females . . . . . 87

4.3 One-way sensitivity analysis results on costs and DALYs for the comparison between the screening strategy and the chloroquine strategy. . . . 92

4.4 Cost-effectiveness plane showing the incremental costs and DALYs averted from the PSA . . . . . . . . . . . . . . . . 94

4.5 Cost-effectiveness acceptability curves . . . . . . . . . . . . . . . . . 94

4.6 One-way sensitivity analysis results on costs and DALYs . . . . . . . . 96

4.7 Two-way sensitivity analysis results showing the impact of changes in the level of adherence to primaquine regimens . . . . . . . . . . . . 98

5.1 Flow diagram of patient recruitment to the trials and inclusion in the household cost study . . . . . . . . . . . . . . . . . . . . 119

5.2 Patterns of treatment seeking among trial participants prior to enrolment in the study . . . . . . . . . . . . . . . . . .

6.1 Flow diagram of $P$. vivax patient case management in the scenario analyses 150

6.2 Global map of P. vivax economic cost burden in the base case . . . . . 155

6.3 Provider, patient and overall cost comparison between the global base case estimate and the scenario analyses . . . . . . . . . . . 156

6.4 Global map of changes in the $P$. vivax cost burden in the scenario analyses as compared to the base case . . . . . . . . . . . . . . 158 


\section{List of Abbreviations}

$\mathrm{ACT}$

AOR

CI

CPI

CrI

DALY

DHP

I\$

ICER

IDR

IMPROV

IQR

FST

G6PD

GDP

MAP

MMV

NMB

OR

PPP

PSA

RDT

SES

SD

SMRU

SP

QALY

US\$

WHO

WHO-CHOICE

WTP artemisinin combination therapy

adjusted odds ratio

confidence interval

consumer price index

credible interval

disability-adjusted life-years

dihydroartemisinin-piperaquine

international dollars

incremental cost-effectiveness ratio

Indonesian rupiah

Improving the radical cure of vivax malaria

interquartile range

fluorescent spot test

glucose-6-phosphate dehydrogenase

gross domestic product

Malaria Atlas Project

Medicines for Malaria Venture

net monetary benefit

odds ratio

purchasing power parity

probabilistic sensitivity analysis

rapid diagnostic test

socio-economic status

standard deviation

Shoklo Malaria Research Unit

sulfadoxine-pyrimethamine

quality-adjusted life-year

United States dollars

World Health Organisation

WHO-CHOosing Interventions that are Cost-Effective

willingness to pay 


\section{Chapter 1}

\section{Introduction}

Malaria is a parasitic disease that has afflicted mankind for more than 100,000 years. Nearly every country in the world was once endemic for malaria, but its range has been reduced significantly since malaria control efforts began in the early 20 th century [1]. Of the five species of malaria that infect humans, the majority of clinical cases are caused by Plasmodium falciparum and Plasmodium vivax. The predominant burden of disease is attributable to P. falciparum, particularly in Sub-Saharan Africa. More recently, however, $P$. vivax has been recognised as an increasingly important cause of morbidity and mortality $[2,3,4,5]$. While $P$. falciparum is more likely to result in direct mortality, multiple relapsing episodes of $P$. vivax can lead to long-term morbidity and indirect mortality $[6,7]$. Despite the increased interest in the clinical and epidemiological consequences of $P$. vivax infections, the associated costs and cost-effectiveness of its diagnosis and treatment has been understudied [8]. Understanding the costs and effects of the diagnosis and treatment of vivax infections is critical to policymakers and healthcare providers with limited resources. This thesis focuses on the costs of $P$. vivax malaria, cost-effectiveness of different treatment options and the global economic burden attributable to the parasite. 


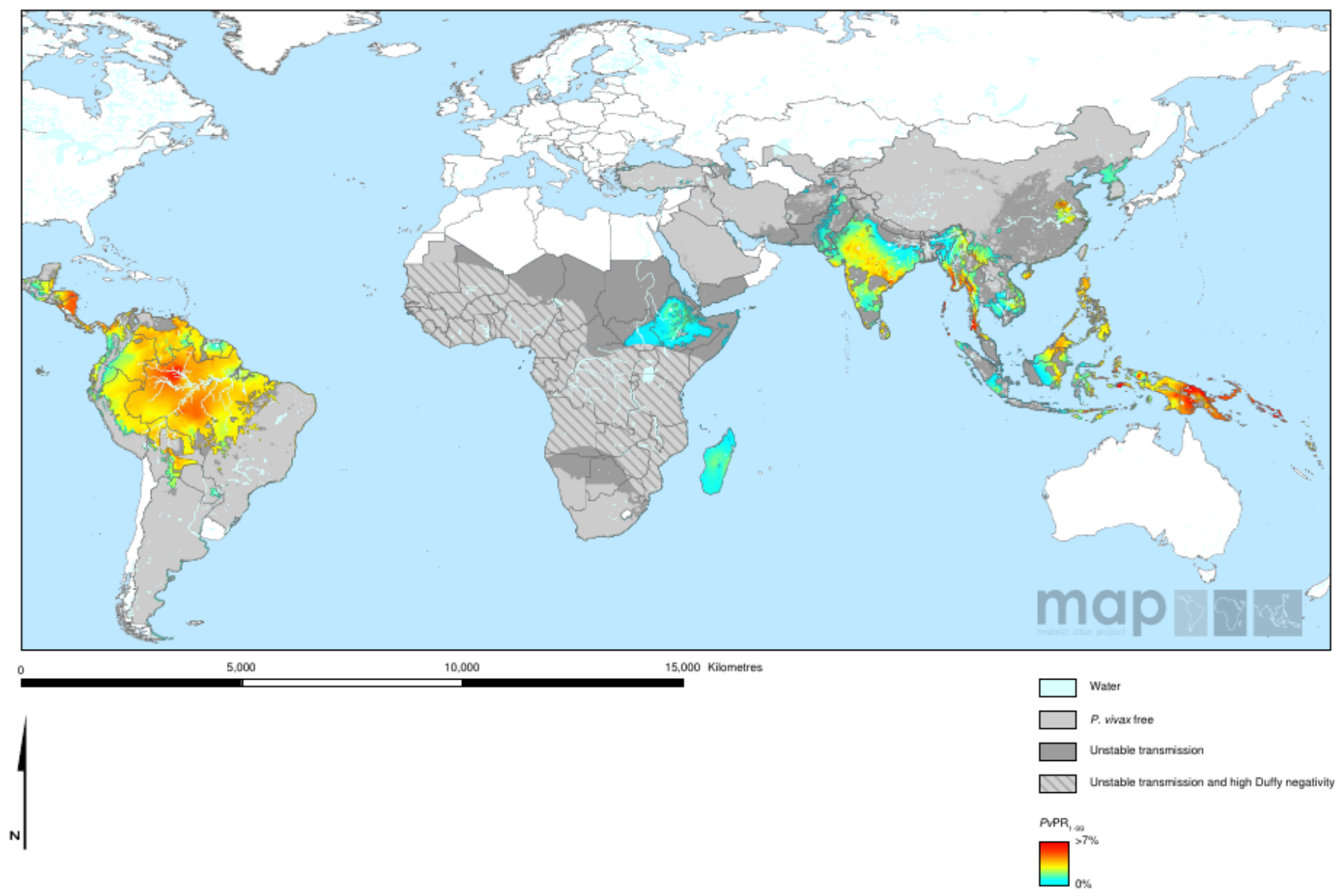

Figure 1.1: The spatial distribution of P. vivax endemicity in 2010. Coloured areas represent the average proportion of the population estimated to be infected with $P$. vivax at any one time over a one year time period. The dark grey areas display where unstable transmission takes place. Downloaded from: http://www.map.ox.ac.uk/browse-resources/endemicity/Pv_mean/world/ [9]. 


\section{$1.1 \quad$ Epidemiology}

While P. vivax rarely occurs in Africa (where the main burden of malaria lies), P. vivax is the predominant cause of malaria in Asia and Central and South America, accounting for more than $50 \%$ and $71-81 \%$ of cases, respectively [2]. The global spatial distribution of vivax malaria is shown in Figure 1.1. A number of important biological differences between $P$. vivax and P. falciparum underpin the variations in their epidemiology and response to malaria control interventions. Perhaps the most important is P. vivax's ability to form dormant liver stages that can recur weeks to months after the primary infection. Furthermore, P. vivax can survive in geographical locations unsuitable to sustaining $P$. falciparum infection. It is spread by a wide range of mosquito vectors, some of which live in temperate climates and bite humans outdoors and during the daytime [4], and transmission occurs early in the parasite's life cycle before the individual becomes symptomatic [10]. These factors make P. vivax much harder to control and eliminate than P. falciparum; hence, in countries reporting fewer than 5,000 cases of malaria per year over $70 \%$ of reported episodes are due to $P$. vivax [11].

An infectious bite by a mosquito injects sporozoites into the host's peripheral blood stream, which then travel to the liver where they invade liver cells (hepatocytes); in turn the cells release thousands of merozoites that initiate the blood stage infection. The blood stage infection results in asexual multiplication until the rising parasitaemia causes a host immune response that causes a cycle of chills and fever that usually repeats every 24 or 48 hours. Other clinical symptoms include headache, anorexia, myalgia, abdominal pain, cough, diarrhoea and anaemia [11]. A key difference from $P$. falciparum, is that once the sporozoites of $P$. vivax and $P$. ovale invade the hepatocytes a proportion of infected cells differentiate to form dormant stages (hypnozoites), that can reactivate weeks or months later to initiate another blood stage infection, which are known as relapses (Figure 1.2) [12, 13].

The frequency and periodicity of $P$. vivax relapses varies significantly between geographical regions. In equatorial locations up to $80 \%$ of patients will experience 


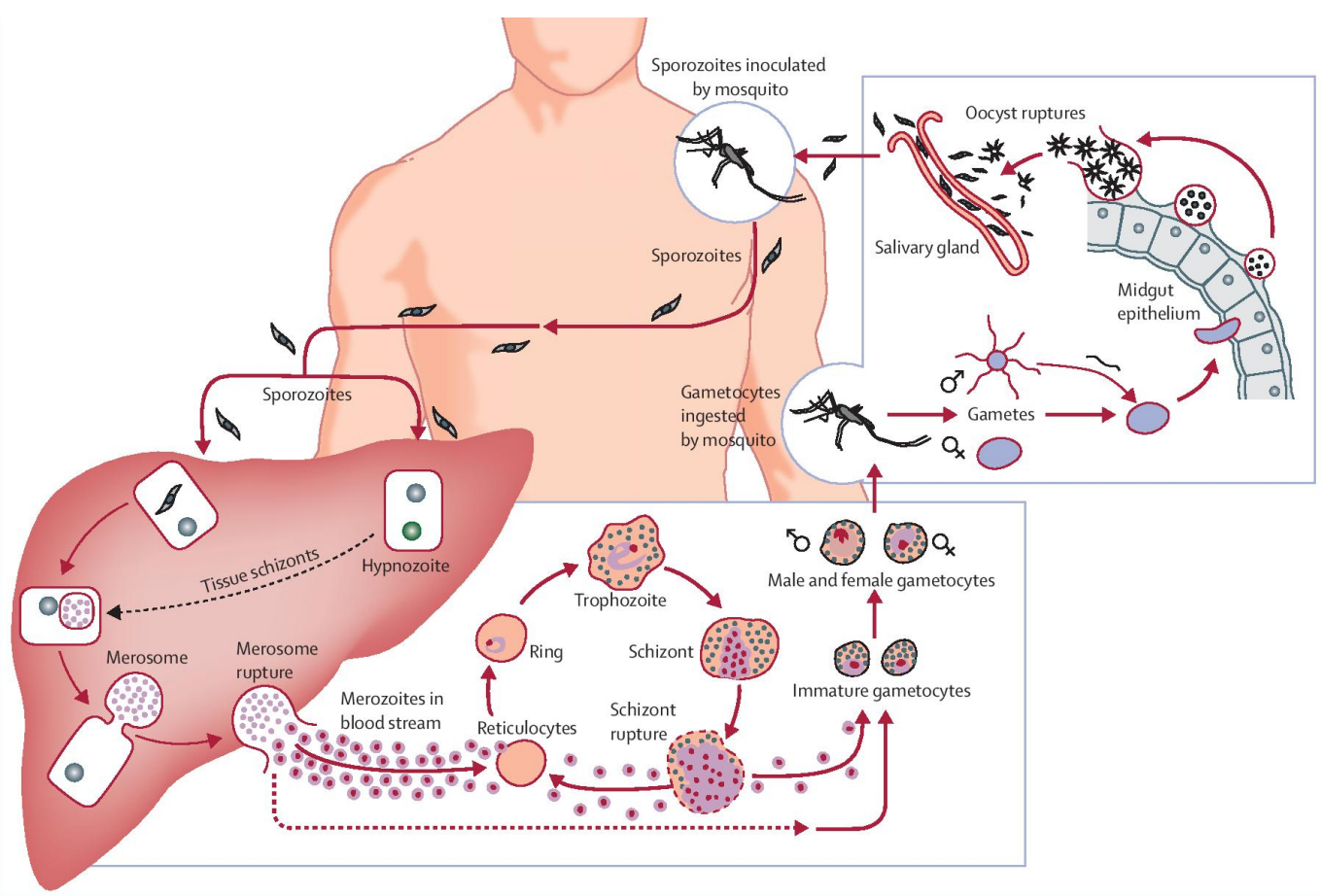

Figure 1.2: The P. vivax life cycle differs from $P$. falciparum in that hypnozoites may be reactivated after the initial infection, causing relapses. Figure from [13].

a relapse and often these occur within six to eight weeks of the primary infection. Whereas in more temperate regions the risk of relapse is much lower and the relapses that do occur, do so much later, often many months after the primary attack [12]. The number of relapses also varies considerably. In military patients returning from high burden areas, soldiers experienced up to 20 relapses over two years [14]. The periodicity of $P$. vivax strains have been categorised in a variety ways $[15,16]$.

In addition to relapsing infections, recurrent parasitaemia following a primary episode of malaria can be due to either reinfection or recrudescence. Reinfection refers to a new episode of malaria originating from another infectious mosquito bite, whereas a recrudescence refers to a parasite surviving in the blood after treatment, and subsequently multiplying after the concentrations of the drug drop below therapeutic levels. While the incidence of any recurrence can be quantified, no reliable method is currently available for discriminating whether the recurrence was due to reinfection, relapse or recrudescences. All recurrences can occur with or without symptoms. A mathematical modelling study in Papua New Guinea estimated that up to $70 \%$ of recurrences were due to relapse, and this figure rose to $96 \%$ in Thailand [17]. A study 
using more recent data from Thailand used a Cox model to estimate that $79 \%$ of recurrences were relapses [18]. This lower percentage is likely due to the longer follow up time and a lower load of hypnozoites associated with a decrease in malaria transmission in the study area.

Where vivax is endemic, immunity develops rapidly, with the peak prevalence occurring in young children and infants $[19,20]$. Young children are at particular risk of anaemia and malnutrition due to repeated episodes caused by relapses [6, 21]. The early ring forms of $P$. vivax and $P$. falciparum are almost identical by microscopy; hence, where $P$. vivax and P. falciparum are co-endemic, it can be difficult to distinguish between the species, particularly as vivax malaria has a lower parasitaemia [5]. These challenges mean that vivax malaria is less likely to be diagnosed and adequately treated.

\subsection{Vivax malaria treatment}

Chloroquine has been the traditional treatment for the blood stages of $P$. vivax and is the usual treatment for acute infections in most vivax endemic countries. High-grade chloroquine resistance in P. falciparum has resulted in a switch in antimalarial policy to artemisinin combination therapy (ACT), which is increasingly used to treat vivax malaria. The difficulties in distinguishing between true parasite recrudescence and other recurrences, have resulted in great difficulty in interpreting $P$. vivax drug efficacy trials, often causing chloroquine resistance to go unnoticed. Using conservative definitions, a meta-analysis suggests that chloroquine-resistant $P$. vivax is widespread and rising $[22,23]$. Several countries have switched to ACT for both species of malaria, including The Solomon Islands, Vanuatu, Papua New Guinea and Indonesia [22, 24].

While ACTs are highly effective at clearing the blood stages of $P$. vivax, they have no efficacy against hypnozoites. An 8-aminoquinoline called primaquine is currently the only licensed drug that can be prescribed for radical cure. Again, because of difficulties distinguishing relapses from reinfections, it is difficult to tell whether resistance to primaquine exists [25]; but a high-dose regimen can prevent over $85 \%$ of recurrent infections when supervised $[26,27]$. The standard treatment regimen for primaquine 
requires pills to be taken over 14 days. While this treatment is generally regarded to be efficacious if all the pills are taken, patient adherence to primaquine is often low due to the lengthy regimen $[28,29]$. While interventions such as directly observed therapy and patient-centred communication training for healthcare providers, have been successful in improving adherence to treatment regimens for diseases like tuberculosis and HIV, fewer interventions have been evaluated for malaria. Two studies in Thailand found directly observed therapy to improve malaria outcomes [30, 31]; however, in Pakistan no difference was seen [32]. Accordingly, interventions to improve adherence will need to be adapted to the setting [33]; designing and evaluating these interventions will be an important objective for $P$. vivax research in the coming years.

Shorter treatment regimens over seven days could improve adherence [34, 35], and a large study is currently underway to assess the safety and non-inferiority of a seven day regimen [36]. Seven day regimens require a higher daily dose which is associated with more gastrointestinal side-effects and, importantly, an increased potential to induce haemolytic anaemia particularly in individuals with glucose-6-phosphate dehydrogenase (G6PD) deficiency.

\subsection{The challenge of G6PD deficiency}

G6PD deficiency is the most common human enzyme defect, ranging in prevalence from less than $1 \%$ to over $30 \%$ of some populations. The prevalence of G6PD deficiency is particularly high in malaria endemic areas [37, 38]. Around 140 variants of G6PD deficiency exist, and the haemolytic risks associated with each variant varies [39]. G6PD is an enzyme that turns carbohydrates into energy, protecting red blood cells from oxidative stress. Individuals with G6PD deficiency either do not have enough of the enzyme or the enzyme does not properly function, and both of these factors result in more rapid death of red blood cells. Nearly 400 million individuals worldwide have G6PD deficiency; these individuals are vulnerable to primaquine-induced haemolysis that gives rise to a range of clinical manifestations from mild to severe and even fatal [39]. 
G6PD deficiency is an x-linked disorder; hence, males have only one copy of the gene and can be hemizygous normal or deficient, whereas females carry two copies of the gene and therefore can be either homozygous normal or deficient, or heterozygous. Due to X-chromosome inactivation, heterozygous females are genetic mosaics with cells randomly inactivating one chromosome or the other, a process known as lyonisation. This process results in a broad spectrum of enzyme activity in females, whilst in males individuals have either normal or very low enzyme activity [39]. The genotype refers to the genetic mutation that causes deficiency, whereas the phenotype, refers to the degree of enzymatic activity that determines the clinical outcome. The classification of enzymatic activity as normal or deficient is determined by population median and varies by location. The risk of haemolysis worsens as the enzyme activity falls, with standard 14 day primaquine regimen often contraindicated in patients with an activity less than 30\%. One recent study, however, demonstrated that female heterozygotes with activity less than 70\% may also have significant haemolysis [40].

Several studies have shown that the prevalence of G6PD deficiency in patients presenting with $P$. vivax malaria is lower than that in the general population; suggesting that G6PD deficiency may confer a degree of protection against malaria [41, 42, 43]. This protection is likely to vary by G6PD variant and parasite strain. In individuals with severe G6PD deficiency, radical cure can be achieved with a weekly dose of primaquine administered for eight weeks [44], although little clinical evidence has been provided regarding the safety and efficacy of such a regimen. Primaquine is also contraindicated in pregnant women due to the unknown G6PD status of the foetus. It is also contraindicated in breastfeeding women due to concerns of excretion of the drug in breast milk; however, recent evidence suggests that the amount of primaquine excreted in the milk is low and thus dangers to the infant may have been exaggerated $[45]$. 


\subsection{Screening for G6PD deficiency and future options for radical cure}

The World Health Organisation (WHO) recommends that primaquine should be used for the radical cure of $P$. vivax patients, but where possible all patients should be tested for G6PD deficiency prior to administration [44], in many malaria endemic communities routine G6PD screening is not undertaken prior to primaquine administration, either due to the lack of suitable point-of-care diagnostics or the cost and logistical difficulties of delivering these. In such areas patients with G6PD deficiency may be put at risk of severe drug-induced haemolysis. While the degree of risk is not well-defined and possibly very low [46], healthcare providers are often reluctant to prescribe primaquine for fear of these immediate adverse effects. In areas where primaquine is not used, patients are at high risk of relapse and a cumulative risk of severe anaemia due to parasite-induced haemolysis. For these reasons, it is difficult to weigh up the risks and benefits of primaquine use.

Other barriers to routine G6PD testing include the additional costs of implementation, the clinical perception that the risk of drug-induced haemolysis is low or that radical cure is not a priority due to the perception that vivax malaria is benign [47]. Spectrophotometry is the gold standard test of G6PD activity, but requires specialised staff and laboratory facilities [48, 49]. Until recently, the only point-of-care test for G6PD deficiency was the fluorescent spot test (FST). The FST requires basic laboratory equipment, rendering it difficult to implement in many field settings. Recently developed qualitative point-of-care G6PD RDTs have a high sensitivity for detecting severe G6PD deficiency, but do not identify those individuals with moderate G6PD deficiency, such as heterozygous females $[50,51,52]$. These currently available G6PD RDTs can be difficult to interpret, potentially lowering their diagnostic accuracy due to misreading the result [53]. Ultimately, the implementation of G6PD RDTs is undermined by the lack of licenses that would allow national malaria control programs to purchase them with global fund subsidy. Until screening tests for G6PD deficiency are widely available 
and affordable, policymakers and health workers in many areas will be reluctant to use primaquine for the treatment of $P$. vivax.

Tafenoquine is another 8-aminoquinoline drug, currently in phase III clinical trials with registration anticipated in 2018. It can achieve radical cure with a single dose, eliminating the issues associated with poor patient adherence to a 14 day primaquine

regimen $[54,55,56]$. While one study showed its haemolytic potential to be similar to 14 day primaquine in heterozygous females, the drug has an increased risk of a prolonged haemolysis due to its longer half-life. Accordingly, the prescription of tafenoquine will demand a quantitative test to screen for G6PD deficiency to rule out patients with an enzyme activity less than $70 \%$ [54].

\subsection{Prospects and challenges for inclusion of $P$. vivax in malaria elimination campaigns}

As discussed above, P. falciparum is more susceptible to traditional control measures, such as bednets (which target mosquitos that bite at night), screening (which is more likely to detect falciparum infections with their higher parastaemia) and treatment for blood stage infections (which ignores the hypnozoite reservoir). These factors result in a greater decrease in P. falciparum than P. vivax in co-endemic areas. Even in areas where the burden of disease is largely due to $P$. vivax, the focus of malaria elimination efforts is usually on P. falciparum. One example is the malaria elimination efforts in the Greater Mekong Subregion. These focus on P. falciparum, due to the urgency engendered by the emergence of artemisinin resistance [57], but had no impact on vivax malaria since radical cure was not given to patients who presented with it [58].

The elimination of vivax malaria therefore presents daunting challenges and any prospects of success will require frequent use of radical treatment for infected individuals, many of whom are asymptomatic. Excluding vivax malaria as a target for elimination could in fact compromise P. falciparum elimination campaigns and efforts to maintain malaria free status. If communities continue to experience cases of malaria, even if due 
to another type of parasite, then it could result in reduced willingness to participate in future elimination activities for the remaining species of malaria and to prevent re-introduction of $P$. falciparum. In an effort to accelerate malaria elimination in the Greater Mekong Subregion, the use of mass drug administration or mass screening and treatment is now being investigated. Alongside these programs, intense community engagement activities are required to communicate the importance of taking antimalarials

to eliminate $P$. falciparum from the community. While campaigns can explain that they are targeting only one type of malaria, it will be challenging to re-engage with these communities after $P$. falciparum elimination if they do not understand the distinction between species. Finally, any delay in the elimination of $P$. vivax will likely require financial and human resources to be sustained over a much longer period of time than if both species was addressed jointly.

\subsection{The intersection of $P$. vivax with health}

\section{economics}

Health economics has great potential to improve the health of populations by optimising the amount of health gained for money spent. While the bulk of research in health economics focuses on the cost of illness and economic evaluation, or the micro-economic impact of alternative strategies for delivering health care, the field of health economics encompasses a much broader remit, most of which is beyond the scope of this thesis but touched upon briefly here. As described by Williams, a number of factors influence health and are influenced by health, including education, wealth, life style and employment conditions [59]. An example of this in vivax malaria is that a single episode can impair educational attainment [60] while a person's education can influence their knowledge about when they should seek treatment for febrile illness because it could be malaria. Poorer individuals are more likely to be at risk of vivax malaria due to occupational hazards (i.e. more time outside due to farming and hunting) while those who have malaria are likely to incur financial and productivity losses from their illness. 
Demand is a particularly thorny issue in that health care is typically needed, rather than wanted, and because of the agency relationship between patients and their healthcare providers. This is a potential conflict of interest in that those who give the expert opinion on what care is needed are also responsible for supplying that care. For P. vivax, this is most obvious in the use of radical cure. In some settings healthcare providers don't prescribe primaquine due to fear of haemolysis in individuals with G6PD deficiency, but in other settings patients are given primaquine without screening for G6PD deficiency because healthcare providers do not see it as enough of a risk. Vivax malaria patients are reliant on their healthcare providers to tell them whether radical cure should be taken and also to provide it for them. Further information assymmetries include the lack of perfect information about the risk of primaquine-induced haemolysis and the risk of recurrence due to $P$. vivax.

The supply of health care involves the cost of delivering care and how different systems of financing health care impact the behaviour of those who supply it. Most of the financing for malaria is vertical and focused on $P$. falciparum instead of $P$. vivax, which can influence how malaria is diagnosed and treated in a community. Countries with $P$. vivax are less likely to receive international support for malaria control than $P$. falciparum [61]. Ideally, supply and demand should balance out through market-clearing prices where the quantity supplied is equal to the quantity demanded, but again, health economics is different. Instead, the rationing of care often occurs through mechanisms such as waiting times and priority-setting criteria. Another potential market failure for vivax malaria is common amongst the infectious diseases: effective treatment and control will have a positive externality in terms of the prevention of onward transmission. Moreover, the planning, budgeting and monitoring mechanisms implemented in a health system will influence the incentives of the stakeholders to discover and implementation of improvements to its efficiency. Evaluations at the whole system level can explore the overall performance, including equity and allocative efficiency (whether the resources spent on health care are producing the most possible health).

Moving to economic evaluation, cost-effectiveness analysis is an approach that 
enables the comparison of different healthcare options by using a common outcome unit. Often, a specific type of cost-effectiveness analysis called a cost-utility analysis is used, enabling comparisons between diseases with a common measure of outcome encompassing both mortality and morbidity, and its implications for quality of life. The two most frequently used outcomes are quality-adjusted life-years (QALYs) and disability-adjust life-years (DALYs). Studies using QALYs seek to maximise them. These are more frequently used in developed countries and involve a tariff that reflects the preferences of the community where the decision is taking place. In contrast to QALYs, the aim is to minimise DALYs. These are applicable across populations and are more commonly used in developing countries.

Regardless of the metric used, the use of cost-effectiveness analyses to evaluate alternative courses of actions is essential to making the most efficient use of scarce resources $[62,63]$. When an intervention saves money while improving outcomes, it "dominates" the alternative; and the decision to adopt should be straightforward. When comparing interventions where one option improves outcomes but costs more, the alternatives can be compared using the incremental cost-effectiveness ratio (ICER), which involves the increase in costs divided by the increase in QALYs or reduction in DALYs. If the ICER is less than the willingness to pay (WTP) threshold, then the intervention is considered to be cost-effective.

The WHO initially recommended that the WTP threshold per DALY averted be set at one to three times the gross domestic product (GDP) per capita [64], but recently re-emphasised that decisions need to be context-specific and that other factors, such as feasibility and budget impact, should also be considered [65]. This guidance is in line with recent studies that have suggested that the WTP threshold should be much lower $[66,67]$. Since the country-level financing for malaria is often a ring-fenced amount of money, one alternative is to use resource allocation based on cost-effectiveness [68]. Another option is to use a form of economic evaluation called cost-benefit analysis where the effects are valuated in monetary terms. When the monetised benefits exceed the costs, then a policy should be implemented. In this way, changes in global health 
expenditures can be compared to options in other sectors.

\subsection{The economics of $P$. vivax treatment}

Although great progress has been made in describing disease outcomes due to P. vivax, many questions remain about the cost-effectiveness of its diagnosis, treatment and management. As previously discussed, the supply of funding for malaria is often focused on $P$. falciparum due to the urgency of the elimination campaigns. A recent literature review by White et al. found only 16 studies that have addressed the costs and cost-effectiveness of vivax interventions [8]. To contrast those numbers, a systematic review by the same author published five years prior found 55 studies on the costs and cost-effectiveness of malaria control, most of which were focused on P. falciparum [69]. When limiting to the diagnosis and treatment of vivax, only nine previous studies were included in White et al.'s review. Three of those studies focused only on diagnostic testing while the remaining included treatment options. The nine studies included a range of percentages of P. vivax cases from $30 \%$ in Papua New Guinea [70] to over 90\% in Afghanistan [71]. The study in Papua New Guinea was the only one to examine a unified treatment of ACT for both P. vivax and P. falciparum, finding it to be a cost-effective option using a 42 day follow up period [70]. To update White et al.'s review, a search using the terms "vivax" and "cost" or "economic": no further relevant studies were found.

The economic burden of $P$. vivax is difficult to determine for a variety of reasons. Cases often occur in rural areas where there are poor healthcare systems and it is often difficult to access care, which can influence treatment-seeking behaviour and the demand for care. Deaths often occur away from facilities, resulting in them not being diagnosed or reported [5]. While it is still commonly believed that these symptoms are only caused by $P$. falciparum, a meta-analysis of $P$. vivax clinical trials listed an extensive number of associated severe outcomes including severe anaemia, shock, multiple convulsions, renal dysfunction, respiratory dysfunction and cerebral malaria [72]. Furthermore, repeated vivax episodes can increase vulnerability to other diseases, such as malnutrition and 
anaemia, and it can be difficult to disentangle these factors $[6,21,73]$.

In addition to morbidity and mortality, the costs associated with treating the disease need to be considered. Costs to the healthcare provider comprise of the direct cost to diagnose and treat a clinical case. When expanding to the societal perspective, the direct costs of seeking treatment for the patient and any companions, which may occur multiple times per episode, and the cost of transport should be included. The indirect costs of disease, including lost productivity, are rarely documented but can be substantial, particularly as children who bear the highest burden of disease will require a caretaker. Finally, the impact of malaria episodes on educational and developmental attainment are increasingly recognized, but again the evidence base for vivax is less established than that for P. falciparum [60,74]. For example, a number of studies have shown the impact of $P$. falciparum on education $[75,76]$, but evidence on the impact of P. vivax on education are scarce.

\subsection{Aims and objectives}

The aim of this thesis is to describe the costs and cost-effectiveness of treatment options for $P$. vivax malaria and the burden of disease associated with it. The specific objectives are the following:

1. Describe the costs due to vivax malaria episodes from the societal and healthcare provider perspectives (Chapters $2 \& 5$ ).

2. Describe the treatment-seeking behaviour and provider compliance following implementation of a universal policy of ACT for all species of malaria in Timika, Indonesia, where a large proportion of cases are due to vivax malaria (Chapter $3)$.

3. Evaluate the cost-effectiveness of screening for G6PD deficiency before treatment with supervised primaquine therapy (Chapter 4).

4. Describe what is currently known about the global economic cost burden due 
to $P$. vivax malaria, the potential costs associated with a worldwide policy of screening before giving radical cure, and the potential cost-benefit associated with a reduction in vivax episodes (Chapter 6).

The thesis is divided into five main chapters (Chapters 2-6) and an overall discussion (Chapter 7 ) that combines the economic analyses undertaken in the preceding chapters and discusses the implications for the allocation of resources required for the diagnosis, treatment, control and ultimately, the elimination of vivax malaria. 


\section{References for Chapter 1}

[1] Bhatt S, Weiss DJ, Cameron E, Bisanzio D, Mappin B, Dalrymple U, et al. The effect of malaria control on Plasmodium falciparum in Africa between 2000 and 2015. Nature. 2015;526(7572):207-211. doi:10.1038/nature15535.

[2] Price RN, Tjitra E, Guerra CA, Yeung S, White NJ, Anstey NM. Vivax malaria: neglected and not benign. Am J Trop Med Hyg. 2007;77(6 Suppl):79-87.

[3] Tjitra E, Anstey NM, Sugiarto P, Warikar N, Kenangalem E, Karyana M, et al. Multidrug-Resistant Plasmodium vivax Associated with Severe and Fatal Malaria: A Prospective Study in Papua, Indonesia. PLoS Med. 2008;5(6):e128.

[4] Battle KE, Gething PW, Elyazar IR, Moyes CL, Sinka ME, Howes RE, et al. The global public health significance of Plasmodium vivax. Adv Parasitol. 2012;80:1-111. doi:10.1016/b978-0-12-397900-1.00001-3.

[5] Baird JK. Evidence and implications of mortality associated with acute Plasmodium vivax malaria. Clin Microbiol Rev. 2013;26(1):36-57. doi:10.1128/cmr.00074-12.

[6] Douglas NM, Pontororing GJ, Lampah DA, Yeo TW, Kenangalem E, Poespoprodjo JR, et al. Mortality attributable to Plasmodium vivax malaria: a clinical audit from Papua, Indonesia. BMC Med. 2014;12:217. doi:10.1186/s12916-014-0217-z.

[7] Moore KA, Simpson JA, Wiladphaingern J, Min AM, Pimanpanarak M, Paw MK, et al. Influence of the number and timing of malaria episodes during pregnancy on prematurity and small-for-gestational-age in an area of low transmission. BMC Med. 2017;15(1):117. doi:10.1186/s12916-017-0877-6.

[8] White MT, Yeung S, Patouillard E, Cibulskis R. Costs and Cost-Effectiveness of Plasmodium vivax Control. Am J Trop Med Hyg. 2016;95(6 Suppl):52-61. doi:10.4269/ajtmh.16-0182. 
[9] Gething PW, Elyazar IR, Moyes CL, Smith DL, Battle KE, Guerra CA, et al. A Long Neglected World Malaria Map: Plasmodium vivax Endemicity in 2010. PLoS Negl Trop Dis. 2012;6(9):e1814. doi:10.1371/journal.pntd.0001814 PNTD-D-12-00489 [pii].

[10] McCarthy JS, Griffin PM, Sekuloski S, Bright AT, Rockett R, Looke D, et al. Experimentally induced blood-stage Plasmodium vivax infection in healthy volunteers. J Infect Dis. 2013;208(10):1688-94. doi:10.1093/infdis/jit394.

[11] World Health Organization. Control and elimination of plasmodium vivax malaria: a technical brief; 2015.

[12] White NJ. Determinants of relapse periodicity in Plasmodium vivax malaria. Malar J. 2011;10:297. doi:1475-2875-10-297 [pii] 10.1186/1475-2875-10-297.

[13] Mueller I, Galinski MR, Baird JK, Carlton JM, Kochar DK, Alonso PL, et al. Key gaps in the knowledge of Plasmodium vivax, a neglected human malaria parasite. Lancet Infect Dis. 2009;9(9):555-66. doi:10.1016/s1473-3099(09)70177-x.

[14] Eyles DE, Young MD. Studies on imported malarias; the parasitological pattern of relapsing Plasmodium vivax in military patients. J Natl Malar Soc. $1948 ; 7(1): 23-37$.

[15] Lover AA, Coker RJ. Quantifying effect of geographic location on epidemiology of Plasmodium vivax malaria. Emerg Infect Dis. 2013;19(7):1058-65. doi:10.3201/eid1907.121674.

[16] Battle KE, Karhunen MS, Bhatt S, Gething PW, Howes RE, Golding N, et al. Geographical variation in Plasmodium vivax relapse. Malar J. 2014;13:144. doi:10.1186/1475-2875-13-144.

[17] Adekunle AI, Pinkevych M, McGready R, Luxemburger C, White LJ, Nosten F, et al. Modeling the dynamics of Plasmodium vivax infection and hypnozoite reactivation in vivo. PLoS Negl Trop Dis. 2015;9(3):e0003595. doi:10.1371/journal.pntd.0003595. 
[18] Chu CS. Factors affecting Plasmodium vivax recurrence. PhD Thesis, The Open University. 2015;.

[19] Maitland K, Williams TN, Bennett S, Newbold CI, Peto TE, Viji J, et al. The interaction between Plasmodium falciparum and P. vivax in children on Espiritu Santo island, Vanuatu. Trans R Soc Trop Med Hyg. 1996;90(6):614-20.

[20] Stanisic DI, Javati S, Kiniboro B, Lin E, Jiang J, Singh B, et al. Naturally acquired immune responses to P. vivax merozoite surface protein 3alpha and merozoite surface protein 9 are associated with reduced risk of P. vivax malaria in young Papua New Guinean children. PLoS Negl Trop Dis. 2013;7(11):e2498. doi:10.1371/journal.pntd.0002498.

[21] Poespoprodjo JR, Fobia W, Kenangalem E, Lampah DA, Hasanuddin A, Warikar N, et al. Vivax Malaria: A Major Cause of Morbidity in Early Infancy. Clin Infect Dis. 2009;48(12):1704-1712.

[22] Douglas NM, Anstey NM, Angus BJ, Nosten F, Price RN. Artemisinin combination therapy for vivax malaria. Lancet Infect Dis. 2010;10(6):405-16. doi:S1473-3099(10)70079-7 [pii] 10.1016/S1473-3099(10)70079-7.

[23] Price RN, von Seidlein L, Valecha N, Nosten F, Baird JK, White NJ. Global extent of chloroquine-resistant Plasmodium vivax: a systematic review and meta-analysis. Lancet Infect Dis. 2014;14(10):982-91. doi:10.1016/s1473-3099(14)70855-2.

[24] Baird KJ, Maguire JD, Price RN. Diagnosis and treatment of Plasmodium vivax malaria. Adv Parasitol. 2012;80:203-70. doi:10.1016/b978-0-12-397900-1.00004-9.

[25] Thomas D, Tazerouni H, Sundararaj KG, Cooper JC. Therapeutic failure of primaquine and need for new medicines in radical cure of Plasmodium vivax. Acta Trop. 2016;160:35-8. doi:10.1016/j.actatropica.2016.04.009.

[26] Nelwan EJ, Ekawati LL, Tjahjono B, Setiabudy R, Sutanto I, Chand K, et al. Randomized trial of primaquine hypnozoitocidal efficacy when administered with 
artemisinin-combined blood schizontocides for radical cure of Plasmodium vivax in Indonesia. BMC Med. 2015;13:294. doi:10.1186/s12916-015-0535-9.

[27] Abreha T, Hwang J, Thriemer K, Tadesse Y, Girma S, Melaku Z, et al. Comparison of artemether-lumefantrine and chloroquine with and without primaquine for the treatment of Plasmodium vivax infection in Ethiopia: A randomized controlled trial. PLoS Med. 2017;14(5):e1002299. doi:10.1371/journal.pmed.1002299.

[28] Bruxvoort K, Goodman C, Kachur SP, Schellenberg D. How patients take malaria treatment: a systematic review of the literature on adherence to antimalarial drugs. PLoS One. 2014;9(1):e84555. doi:10.1371/journal.pone.0084555.

[29] Douglas NM, Poespoprodjo JR, Patriani D, Malloy MJ, Kenangalem E, Sugiarto P, et al. Unsupervised primaquine for the treatment of Plasmodium vivax malaria relapses in southern Papua: A hospital-based cohort study. PLoS Med. 2017;14(8):e1002379. doi:10.1371/journal.pmed.1002379.

[30] Maneeboonyang W, Lawpoolsri S, Puangsa-Art S, Yimsamran S, Thanyavanich N, Wuthisen P, et al. Directly observed therapy with primaquine to reduce the recurrence rate of plasmodium vivax infection along the Thai-Myanmar border. Southeast Asian J Trop Med Public Health. 2011;42(1):9-18.

[31] Takeuchi R, Lawpoolsri S, Imwong M, Kobayashi J, Kaewkungwal J, Pukrittayakamee S, et al. Directly-observed therapy (DOT) for the radical 14-day primaquine treatment of Plasmodium vivax malaria on the Thai-Myanmar border. Malar J. 2010;9:308. doi:10.1186/1475-2875-9-308.

[32] Leslie T, Rab MA, Ahmadzai H, Durrani N, Fayaz M, Kolaczinski J, et al. Compliance with 14-day primaquine therapy for radical cure of vivax malaria-a randomized placebo-controlled trial comparing unsupervised with supervised treatment. Trans R Soc Trop Med Hyg. 2004;98(3):168-73. 
[33] Thriemer K, Ley B, Bobogare A, Dysoley L, Alam MS, Pasaribu AP, et al. Challenges for achieving safe and effective radical cure of Plasmodium vivax: a round table discussion of the APMEN Vivax Working Group. Malar J. 2017;16(1):141. doi:10.1186/s12936-017-1784-1.

[34] Krudsood S, Tangpukdee N, Wilairatana P, Phophak N, Baird JK, Brittenham GM, et al. High-dose primaquine regimens against relapse of Plasmodium vivax malaria. Am J Trop Med Hyg. 2008;78(5):736-40.

[35] Pareek A, Chandurkar N, Gogtay N, Deshpande A, Kakrani A, Kaneria M, et al. Sustained Release Formulation of Primaquine for Prevention of Relapse of Plasmodium vivax Malaria: A Randomized, Double-Blind, Comparative, Multicentric Study. Malar Res Treat. 2015;2015:579864. doi:10.1155/2015/579864.

[36] IMPROV Study Group. Improving the radical cure of vivax malaria (IMPROV): a study protocol for a multicentre randomised, placebo-controlled comparison of short and long course primaquine regimens. BMC Infect Dis. 2015;15:558. doi:10.1186/s12879-015-1276-2.

[37] Nkhoma ET, Poole C, Vannappagari V, Hall SA, Beutler E. The global prevalence of glucose-6-phosphate dehydrogenase deficiency: a systematic review and meta-analysis. Blood Cells Mol Dis. 2009;42(3):267-78. doi:S1079-9796(09)00005-9 [pii] 10.1016/j.bcmd.2008.12.005.

[38] Howes RE, Piel FB, Patil AP, Nyangiri OA, Gething PW, Dewi M, et al. G6PD deficiency prevalence and estimates of affected populations in malaria endemic countries: a geostatistical model-based map. PLoS Med. 2012;9(11):e1001339. doi:10.1371/journal.pmed.1001339.

[39] Cappellini MD, Fiorelli G. Glucose-6-phosphate dehydrogenase deficiency. Lancet. 2008;371(9606):64-74. doi:10.1016/s0140-6736(08)60073-2.

[40] Chu CS, Bancone G, Moore KA, Win HH, Thitipanawan N, Po C, et al. Haemolysis in G6PD Heterozygous Females Treated with Primaquine for 
Plasmodium vivax Malaria: A Nested Cohort in a Trial of Radical Curative Regimens. PLoS Med. 2017;14(2):e1002224. doi:10.1371/journal.pmed.1002224.

[41] Louicharoen C, Patin E, Paul R, Nuchprayoon I, Witoonpanich B, Peerapittayamongkol C, et al. Positively selected G6PD-Mahidol mutation reduces Plasmodium vivax density in Southeast Asians. Science. 2009;326(5959):1546-9. doi:10.1126/science.1178849.

[42] Khim N, Benedet C, Kim S, Kheng S, Siv S, Leang R, et al. G6PD deficiency in Plasmodium falciparum and Plasmodium vivax malaria-infected Cambodian patients. Malar J. 2013;12:171. doi:10.1186/1475-2875-12-171.

[43] Uyoga S, Ndila CM, Macharia AW, Nyutu G, Shah S, Peshu N, et al. Glucose-6-phosphate dehydrogenase deficiency and the risk of malaria and other diseases in children in Kenya: a case-control and a cohort study. Lancet Haematol. 2015;2(10):e437-44. doi:10.1016/s2352-3026(15)00152-0.

[44] World Health Organisation. Guidelines for the Treatment of Malaria. 2015;

[45] Gilder ME, Hanpithakphong W, Hoglund RM, Tarning J, Win HH, Hilda N, et al. Primaquine Pharmacokinetics in Lactating Women and Breastfed Infant Exposures. Clinical Infectious Diseases. 2018; p. ciy235-ciy235. doi:10.1093/cid/ciy235.

[46] Ashley EA, Recht J, White NJ. Primaquine: the risks and the benefits. Malar J. 2014;13:418. doi:10.1186/1475-2875-13-418.

[47] Ley B, Luter N, Espino FE, Devine A, Kalnoky M, Lubell Y, et al. The challenges of introducing routine G6PD testing into radical cure: a workshop report. Malaria Journal. 2015;14(1):377.

[48] Luzzatto L, Seneca E. G6PD deficiency: a classic example of pharmacogenetics with on-going clinical implications. Br J Haematol. 2014;164(4):469-80. doi:10.1111/bjh.12665. 
[49] Brito MA, Peixoto HM, Almeida AC, Oliveira MR, Romero GA, Moura-Neto JP, et al. Validation of the rapid test Carestart $^{\mathrm{TM}}$ G6PD among malaria vivax-infected subjects in the Brazilian Amazon. Rev Soc Bras Med Trop. 2016;49(4):446-55. doi:10.1590/0037-8682-0134-2016.

[50] Roca-Feltrer A, Khim N, Kim S, Chy S, Canier L, Kerleguer A, et al. Field trial evaluation of the performances of point-of-care tests for screening G6PD deficiency in Cambodia. PLoS One. 2014;9(12):e116143. doi:10.1371/journal.pone.0116143.

[51] Bancone G, Chu CS, Chowwiwat N, Somsakchaicharoen R, Wilaisrisak P, Charunwatthana $\mathrm{P}$, et al. Suitability of capillary blood for quantitative assessment of G6PD activity and performances of G6PD point-of-care tests. Am J Trop Med Hyg. 2015;92(4):818-24. doi:10.4269/ajtmh.14-0696.

[52] Satyagraha AW, Sadhewa A, Elvira R, Elyazar I, Feriandika D, Antonjaya U, et al. Assessment of Point-of-Care Diagnostics for G6PD Deficiency in Malaria Endemic Rural Eastern Indonesia. PLoS Negl Trop Dis. 2016;10(2):e0004457. doi:10.1371/journal.pntd.0004457.

[53] Oo NN, Bancone G, Maw LZ, Chowwiwat N, Bansil P, Domingo GJ, et al. Validation of G6PD Point-of-Care Tests among Healthy Volunteers in Yangon, Myanmar. PLoS One. 2016;11(4):e0152304. doi:10.1371/journal.pone.0152304.

[54] Llanos-Cuentas A, Lacerda MV, Rueangweerayut R, Krudsood S, Gupta SK, Kochar SK, et al. Tafenoquine plus chloroquine for the treatment and relapse prevention of Plasmodium vivax malaria (DETECTIVE): a multicentre, double-blind, randomised, phase $2 \mathrm{~b}$ dose-selection study. Lancet. 2014;383(9922):1049-58. doi:10.1016/s0140-6736(13)62568-4.

[55] GlaxoSmithKline. Study to Assess the Incidence of Hemolysis, Safety, and Efficacy of Tafenoquine (SB-252263, WR238605) Versus Primaquine in Subjects With Plasmodium Vivax Malaria. National Library of Medicine (US). 
2014;Available from: https://clinicaltrials.gov/ct2/show/NCT02216123 NLM Identifier: NCT02216123(Accessed: 2017 August 17).

[56] GlaxoSmithKline. Ph 2B/3 Tafenoquine (TFQ) Study in Prevention of Vivax Relapse. National Library of Medicine (US). 2011;Available from: https://clinicaltrials.gov/ct2/show/NCT01376167 NLM Identifier: NCT 01376167(Accessed: 2017 August 17).

[57] World Health Organization. Strategy for Malaria Elimination in the Greater Mekong Subregion (2015-2030). 2015;.

[58] Landier J, Kajeechiwa L, Thwin MM, Parker DM, Chaumeau V, Wiladphaingern J, et al. Safety and effectiveness of mass drug administration to accelerate elimination of artemisinin-resistant falciparum malaria: A pilot trial in four villages of Eastern Myanmar. Wellcome Open Res. 2017;2:81. doi:10.12688/wellcomeopenres.12240.1.

[59] Williams A. Being Reasonable About the Economics of Health: Selected Essays by Alan Williams. Cheltenham: Edward Elgar. 1997;

[60] Vitor-Silva S, Reyes-Lecca RC, Pinheiro TR, Lacerda MV. Malaria is associated with poor school performance in an endemic area of the Brazilian Amazon. Malar J. 2009;8:230. doi:10.1186/1475-2875-8-230.

[61] Pigott DM, Atun R, Moyes CL, Hay SI, Gething PW. Funding for malaria control 2006-2010: a comprehensive global assessment. Malar J. 2012;11:246. doi:10.1186/1475-2875-11-246.

[62] Cairns J. Using Cost-Effectiveness Evidence to Inform Decisions as to which Health Services to Provide. Health Systems \& Reform. 2016;2(1):32-38. doi:10.1080/23288604.2015.1124172.

[63] Paulden M, O’Mahony J, McCabe C. Determinants of Change in the Cost-effectiveness Threshold. Med Decis Making. 2017;37(2):264-276. doi:10.1177/0272989x16662242. 
[64] World Health Organization. Macroeconomics and health: investing in health for economic development. Report of the Commission on Macroeconomics and Health. World Health Organization; 2001. Available from: http://apps . who.int/iris/bitstream/10665/42435/1/924154550X.pdf.

[65] Bertram MY, Lauer JA, De Joncheere K, Edejer T, Hutubessy R, Kieny MP, et al. Cost-effectiveness thresholds: pros and cons. Bull World Health Organ. 2016;94(12):925-930. doi:10.2471/blt.15.164418.

[66] Marseille E, Larson B, Kazi DS, Kahn JG, Rosen S. Thresholds for the cost-effectiveness of interventions: alternative approaches. Bull World Health Organ. 2015;93(2):118-24. doi:10.2471/blt.14.138206.

[67] Woods B, Revill P, Sculpher M, Claxton K. Country-Level Cost-Effectiveness Thresholds: Initial Estimates and the Need for Further Research. Value Health. 2016;19(8):929-935. doi:10.1016/j.jval.2016.02.017.

[68] Drake TL, Lubell Y, Kyaw SS, Devine A, Kyaw MP, Day NPJ, et al. Geographic Resource Allocation Based on Cost Effectiveness: An Application to Malaria Policy. Appl Health Econ Health Policy. 2017;15(3):299-306. doi:10.1007/s40258-017-0305-2.

[69] White MT, Conteh L, Cibulskis R, Ghani AC. Costs and cost-effectiveness of malaria control interventions-a systematic review. Malar J. 2011;10:337. doi:10.1186/1475-2875-10-337.

[70] Davis WA, Clarke PM, Siba PM, Karunajeewa HA, Davy C, Mueller I, et al. Cost-effectiveness of artemisinin combination therapy for uncomplicated malaria in children: data from Papua New Guinea. Bull World Health Organ. 2011;89(3):211-20. doi:10.2471/blt.10.084103.

[71] Hansen KS, Grieve E, Mikhail A, Mayan I, Mohammed N, Anwar M, et al. Cost-effectiveness of malaria diagnosis using rapid diagnostic tests compared to 
microscopy or clinical symptoms alone in Afghanistan. Malar J. 2015;14:217. doi:10.1186/s12936-015-0696-1.

[72] Rahimi BA, Thakkinstian A, White NJ, Sirivichayakul C, Dondorp AM, Chokejindachai W. Severe vivax malaria: a systematic review and meta-analysis of clinical studies since 1900. Malar J. 2014;13:481. doi:10.1186/1475-2875-13-481.

[73] Kenangalem E, Karyana M, Burdarm L, Yeung S, Simpson JA, Tjitra E, et al. Plasmodium vivax infection: a major determinant of severe anaemia in infancy. Malar J. 2016;15:321. doi:10.1186/s12936-016-1373-8.

[74] Chen I, Clarke SE, Gosling R, Hamainza B, Killeen G, Magill A, et al. "Asymptomatic" Malaria: A Chronic and Debilitating Infection That Should Be Treated. PLoS Med. 2016;13(1):e1001942. doi:10.1371/journal.pmed.1001942.

[75] Nankabirwa J, Wandera B, Kiwanuka N, Staedke SG, Kamya MR, Brooker SJ. Asymptomatic Plasmodium infection and cognition among primary schoolchildren in a high malaria transmission setting in Uganda. Am J Trop Med Hyg. 2013;88(6):1102-8. doi:10.4269/ajtmh.12-0633.

[76] Boivin MJ, Sikorskii A, Familiar-Lopez I, Ruisenor-Escudero H, Muhindo M, Kapisi J, et al. Malaria illness mediated by anaemia lessens cognitive development in younger Ugandan children. Malar J. 2016;15:210. doi:10.1186/s12936-016-1266-x. 


\section{Chapter 2}

\section{Treatment-seeking behaviour and}

\section{associated costs for malaria in}

\section{Papua, Indonesia}

This chapter has been published as Karyana $\mathrm{M}^{*}$, Devine $\mathrm{A}^{*}$, Kenangalem E, Burdarm L, Poespoprodjo JR, Vemuri R, Anstey NM, Tjitra E, Price RN, Yeung S. Treatmentseeking behaviour and associated costs for malaria in Papua, Indonesia. Malar J. 2016;15(1):536. https://doi.org/10.1186/s12936-016-1588-8. PMID: 27821127. * Joint first authors.

\section{$2.1 \quad$ Introduction}

Great progress has been made in reducing the burden of malaria in the Asia Pacific region, however the ultimate goal of eliminating malaria has been undermined by the emergence and spread of drug resistance. The WHO recommends that once antimalarial efficacy falls below 90\%, treatment policy should be changed with a combination therapy with greater than $95 \%$ efficacy [1].

The impact of any antimalarial drug policy will depend on how it is implemented, and whether it ensures that individuals with malaria can easily access and receive the 
right treatment and diagnostics at an affordable cost. Early diagnosis of individuals with malaria and administration of effective treatment is critical both to individual patients in ensuring rapid resolution of symptoms and preventing progression to severe disease, but also the community, by reducing further transmission and spread of antimalarial drug resistance.

In many malaria-endemic countries, the private sector is an important source of care for individuals seeking treatment for malaria and most of the spending is out-of-pocket. The private sector comprises a diverse range of providers including private hospitals, registered pharmacies and unlicensed drug shops. Having an understanding of the role of different types of healthcare providers and engaging with them appropriately is therefore very important if changes in policy are to result in actual change in how patients with malaria are treated. Treatment-seeking behaviour of patients with symptomatic malaria is influenced by socio-economic factors, knowledge and beliefs, and access to healthcare provision. Malaria disproportionately affects rural poor households for whom one episode of illness can have catastrophic results, not only in terms of morbidity and mortality but also in terms of financial burden.

The literature on treatment-seeking behaviour for malaria, has shown that individuals often begin by taking treatment at home or from traditional healers and then move on to private drug sellers with few accessing public facilities directly $[2,3]$. In Sub-Saharan Africa, which carries the highest burden of malaria, studies have shown that less than $25 \%$ of the private providers have malaria tests available $[4,5]$. In Asia, a recent household survey in Cambodia showed that two-thirds of individuals seek treatment at private providers [6]. Another survey in Myanmar revealed that nearly half of those who sought treatment did so at private providers where they were less likely to receive a diagnostic test for malaria and more likely to receive antimalarials without a diagnosis $[7]$.

The greatest burden of malaria in Indonesia is in the eastern provinces including Papua, where P. falciparum and P. vivax are the dominant species [8, 9]. Malaria outbreaks are frequent, often triggered by the migration of non-immune individuals 
from low to high transmission areas [10]. Prior to 2006, the first-line treatment of uncomplicated P. falciparum malaria was chloroquine plus sulfadoxine-pyrimethamine (SP) and that for patients with uncomplicated $P$. vivax was chloroquine with 14 days of unsupervised primaquine (total dose $3.5 \mathrm{mg} / \mathrm{kg}$ over 14 days), for patients over one year of age [11]. A series of antimalarial efficacy studies conducted in 2004 and 2005, documented high levels of resistance to both chloroquine and SP $[12,13]$.

At the time of this household survey, little data were available on treatment-seeking behaviour, antimalarial drug use and household costs of treatment for malaria in Indonesia, where malaria remains a significant public health concern $[14,15]$. A household survey in 2002-2003 indicated that less than 1\% of children under the age of five received an antimalarial for fever [15]. Another household survey conducted in Java in 2006 showed that the public sector was the primary source of malaria treatment for less than half of those with self-reported malaria [14]. Although several treatmentseeking surveys have been conducted in Asia [6, 7, 14, 16, 17, 18, 19, 20, 21, 22], almost no data exists on the associated healthcare costs for P. falciparum and P. vivax malaria in a co-endemic area as only one reported costs associated with each malarial episode with little detail on what these costs entailed [14].

The aims of the current study were (1) to describe treatment-seeking behaviour for fever and (2) to describe the associated costs of $P$. vivax and $P$. falciparum infection in Papua, prior to March 2006, when policy was changed to dihydoartemisinin-piperaquine (a fixed dose combination therapy) for uncomplicated malaria due to any species of malaria. A subsequent household survey to determine changes in treatment seeking and costs was conducted in 2013 and is reported in Chapter 3.

\subsection{Methods}

\subsubsection{Site details}

The survey was conducted in Mimika Regency, southern Papua, Indonesia. This district covers an area of $21,522 \mathrm{~km}^{2}$ with approximately 130,000 people living in 12 districts in 
2004. Most of the population resides in Timika town and the surrounding villages. The area is inhabited by a variety of ethnic groups which are broadly categorized as lowland Papuans, highland Papuans or non-Papuans. The demographics, malaria transmission and health services in this region have been described previously [8].

The area is covered with thick rain forest with both coastal and mountainous areas. Malaria transmission occurs throughout the year and is restricted to the lowland area where most of the population lives. Immigration to work at the local mine is considerable and has the potential both to expose non-immune individuals to malaria and to introduce malaria from other areas of Indonesia. There are three mosquito vectors: Anopheles koliensis, Anopheles farauti and Anopheles punctulatus. Based on the malaria surveillance data, the annual incidence of clinical malaria, is estimated to be approximately 876 episodes per 1,000 people, with a slight predominance of $P$. falciparum infection (46\%) compared to P. vivax (39\%) [8].

Healthcare provision is provided free of charge at the hospital, 12 Government-funded Primary Health Clinics (Puskesmas) and, for those employed by a local mining company, at the malaria control clinics where parasitological diagnosis by microscopy, and rapid diagnostic test are available. There is also a thriving private sector which includes clinics, pharmacies and drug stores. Whilst antimalarials are officially prescription drugs, they can also be purchased over the counter in the private sector [10]. The malaria surveillance program does not capture the malaria cases seen in the private sector.

\subsubsection{Household sampling strategy}

The survey was conducted in the Mimika district between July and December 2005.

The sample size calculation aimed to estimate the true prevalence of households with someone with a recent history of fever and was based on an estimated prevalence of $40 \%$ and population size of 140,000 ; a multiplication factor of two was used to take into account the design effect due to cluster sampling. A sample size of 800 households was required to achieve an estimate of prevalence with greater than $95 \%$ confidence. 
A three-stage cluster sampling procedure was applied to identify 32 clusters of 25 households [23]. Firstly the four most populous subdistricts (Mimika Baru, Mimika Timur, Kuala Kencana and Tembaga Pura) were chosen from the 12 subdistricts based on their accessibility by road from the main town of Timika and because they included the majority $(80 \%)$ of the districts population. Secondly, the number of clusters in each subdistrict was apportioned according to their relative populations. The houses were then assigned numbers according to their geographical location and clusters of 25 houses were identified randomly according to WHO guidelines with the first house identified by random allocation.

Members of the households were defined as all individuals living under one roof, who ate from one kitchen and had resided in the study area for at least six months. If a selected household was empty, the interviewer returned the next day. If still unoccupied, the next occupied household was sampled instead. Temporary migrants were defined as individuals who had moved into the area within last six months and did not plan to stay longer than six months; these were excluded from the survey.

\subsubsection{Data collection}

The survey was conducted using a structured questionnaire capturing self-reported household demographic data, income, average monthly food and non-food expenditures, expenditure on healthcare in the last month, ownership of assets, and bed net ownership and use. Nearly all surveys were conducted in the local language, Bahasa Indonesian. The survey was conducted with the head of the household or another suitable adult and details on all household members gathered. Those members who were present were asked specific details about recent illness, had a physical examination and were asked to consent to a blood film as previously described [8].

In order to obtain detailed information about the care-seeking process and cost of illness, individuals reporting a history of fever in the preceding 30 days were asked to complete a separate module on treatment seeking, comprising of questions on all the places that they went to seek treatment, whether they received a blood test and 
the type of medicines they received. They were also asked about direct and indirect household costs including the cost of treatment, transport, productivity losses due to illness, and companion, caretaking and substitute labour [21].

An exit survey was also undertaken on patients with confirmed malaria as they left public facilities in order to collect accurate information on household costs in relation to type (species) of malaria infection. The exit interviews were carried out over a period of four months in the hospital, two of the four Puskesmas and three of the eight malaria control clinics. The number of interviews per type of facility was proportional to the number of malaria patients each facility saw each month. Any patient with a parasitological diagnosis of malaria was eligible for inclusion. Patients completed a questionnaire with the aid of a research nurse to document treatment seeking and costs of illness. With a shorter time between the event and the survey, these results were less likely to be confounded by recall bias and enabled a greater certainty about malaria species diagnosis with the disadvantage of truncating the costs at the end of the healthcare visit.

\subsubsection{Data analysis}

Data were analysed using STATA statistical software (version 14) [24] and R (version 3.2.3) [25]. Frequencies and percentages were used for the descriptive data. Percentages were compared using the chi-square test $\left(X^{2}\right)$. Differences in outcome distributions were tested using Mann Whitney test for two groups and the Kruskal Wallis H test for more than two groups. Continuous variables were compared using Spearmans rank for correlation. Simple logistic regression was used to calculate odds ratios (ORs). The variables that were significant risk factors $(\mathrm{p}<0.05)$ for fever in the past month in the univariate analysis were included in a multiple logistic regression model. Regression models involving behaviour of the individuals were adjusted for clustering by households, since more than one household member could have been present for each household. 


\section{Treatment-seeking behaviour}

For the analysis on treatment-seeking behaviour, those who reported that their fever started in the last two days were excluded in order to ensure that only individuals who had had sufficient time to seek or not seek treatment were included. For those included in the treatment-seeking behaviour analysis, the following outcomes were calculated: the percentage that reported taking any medicines (including at home or traditional medicine) and percentage seeking treatment outside of the home according to the number and type of provider, categorised into private providers (private clinics, pharmacies and drug shops) and public facilities (the hospital, Puskesmas and malaria control clinics).

In order to explore how patients were managed at each provider in terms of blood testing and prescriptions of antimalarials, further analysis was undertaken on each patient-provider interaction (visit). A visit could be inpatient or outpatient. As antimalarial injections pose the risk of infection to patients and are largely inappropriate treatments for uncomplicated malaria, individuals were also asked whether they received an injection or tablets or both.

\section{Socio-economic status}

In order to categorise the surveyed by SES, a Discriminant Analysis of Principal Components [26] was used to analyse the data on reported household ownership of assets. This approach maximized between group variability while minimising within group variability, capturing heterogeneity in ownership that was not seen using a standard principal component analysis [27]. In order to investigate the relationship between SES and other outcomes captured by the survey, the groups were ranked from poorest to richest in terms of both income and expenditure per household member.

\section{Costs}

Costs were gathered in Indonesian rupiah (IDR), and converted into United States Dollars (US\$) using the average exchange rate for 2005 [28] and then revised to the 
2014 equivalent using the consumer price index for Indonesia [29]. To represent the cost burden on households, the mean and standard deviation (SD) are reported. As the data were skewed, the Mann-Whitney test was used for comparisons, and the median and interquartile range (IQR) are also included. Direct costs, including consultation, diagnosis, medication, overnight stays, administration and transportation, were reported according to where treatment was obtained [30]. Indirect costs, including lost wages for companions, caretakers and substitute labourers, are aggregated into cost per individual taking treatment. As a result, when multiple companions, caretakers or substitute labourers were reported for a single individual taking treatment, their times were summed, resulting in the mean days per individual taking treatment. The mean wage was calculated for all who reported a wage, including individuals taking treatment, companions, caretakers and substitute labourers. This wage was applied to all who reported reducing their activities because they had a fever or were a companion, caretaker or substitute labourer for someone with a fever. While this may have overestimated the losses for individuals who are unpaid for their labour (i.e. housework), it attempts to capture the productivity losses for those individuals and the households that rely on them. For those who reported reducing some of their usual activities, the days of lost activity were multiplied by one half day's wage and included in the total costs.

\section{$2.3 \quad$ Results}

Table 2.1 describes the questionnaires, sample sizes and dates included in the surveys.

\subsubsection{Household demographics, SES and expenditure}

In total 825 households with 5,255 individuals were included in the survey between July and December 2005 (Figure 2.1). The median household size was six individuals (IQR, 4-8) with a maximum of 24 in one house. The median duration that a household had lived at that location was nine years (IQR, 4-15). Thirty four (4\%) of households reported moving into that location less than a year previously. Of the 763 households 
Table 2.1: Surveys, questionnaires included in the surveys, sample sizes and dates.

\begin{tabular}{llll}
\hline Survey & Questionnaire & Sample size & Dates \\
\hline Household & Socio-demographic details & $825^{*}$ & June to November 2005 \\
Household & Household members & 5255 & June to November 2005 \\
Household & Fever in past month \& parasite positivity & 3896 & June to November 2005 \\
Household & Treatment seeking & 1176 & June to November 2005 \\
Household & Costs & 1176 & June to November 2005 \\
Hospital Exit & Socio-demographic details & 358 & May to November 2005 \\
Hospital Exit & Treatment seeking & 358 & May to November 2005 \\
Hospital Exit & Costs & 358 & May to November 2005 \\
\hline
\end{tabular}

* This is the number of households. All other sample sizes are for individuals.

in which income was recorded, 63\% (482) reported a total monthly income of less than US\$500.

For the SES, the Discriminant Analysis of Principal Components resulted in households being split into five groups ranging in size from 111 to 203. Richer households had electricity and owned electrical items but did not always own a house or land, whereas poorer households tended to own houses, land and grow crops, but were less likely to have electricity (Figure 2.2). Household SES was significantly lower in Highland Papuan household with $47 \%$ (101/216) of Highland Papuans in the poorest categories compared to $12 \%(22 / 190)$ of Lowland Papuans and $4 \%(16 / 416)$ of non-Papuans $(\mathrm{p}<0.001)$. The household general health expenditure in the previous month was significantly lower in households with lower SES ( $\mathrm{p}<0.001$; Figure 2.3). The estimated household general health expenditure was correlated with the number of individuals reporting fever in a household $(\rho=0.209, \mathrm{p}<0.001)$.

Only $340(41 \%)$ of households reported owning a bed net, and this was inversely correlated with SES with 51\% (72/140) in the poorest group reported bed net ownership as compared to $29 \%(32 / 111)$ in the richest $(\mathrm{p}<0.001)$. 


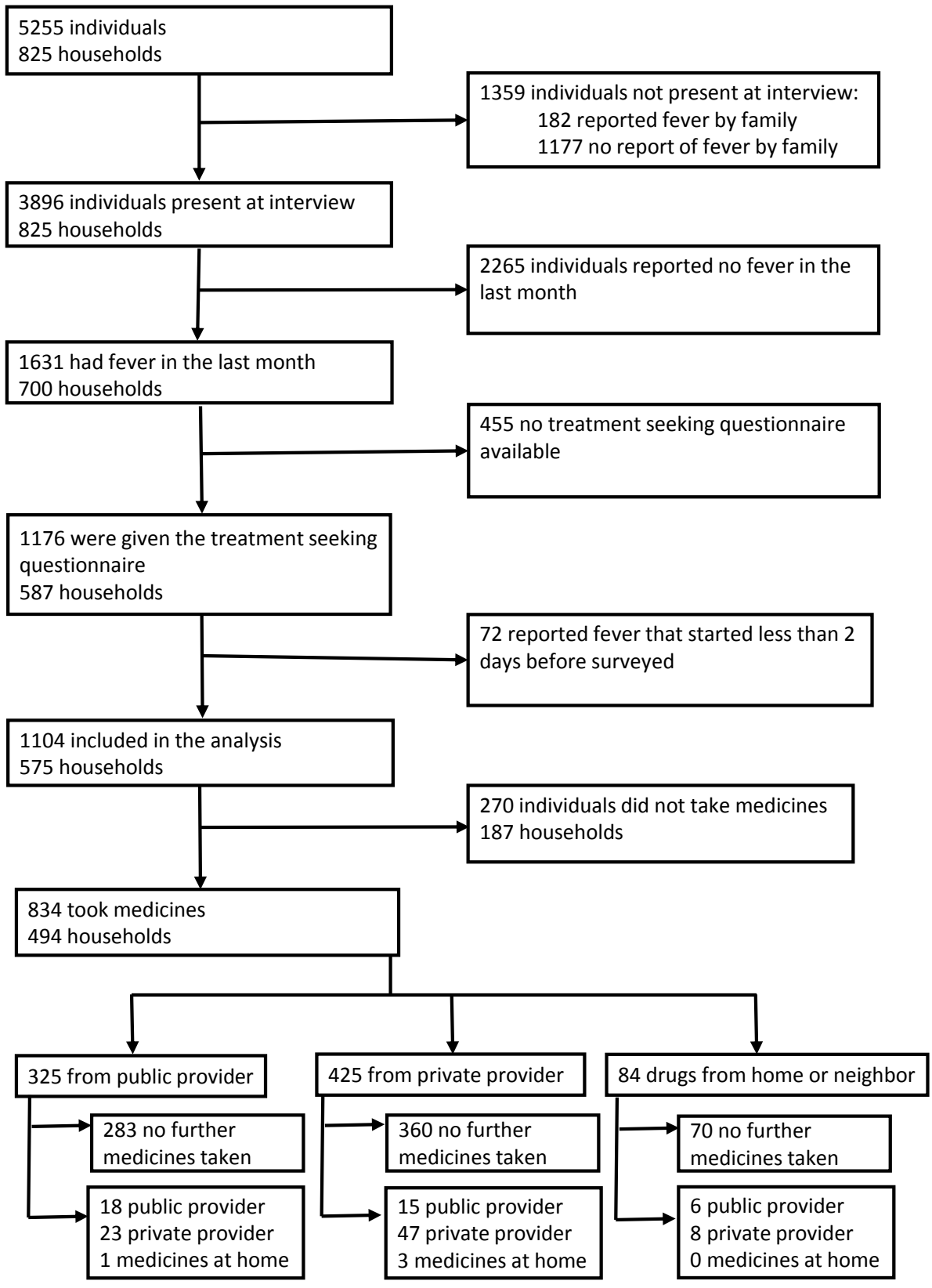

Figure 2.1: Flow Diagram of household members, interviews, initial location of treatment taking, and whether took a second treatment. 
a)

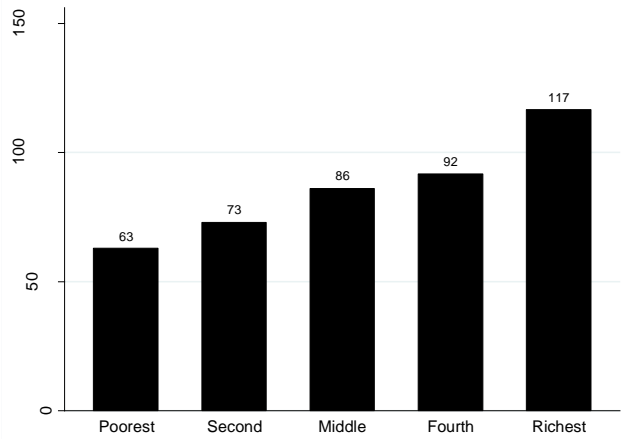

b)

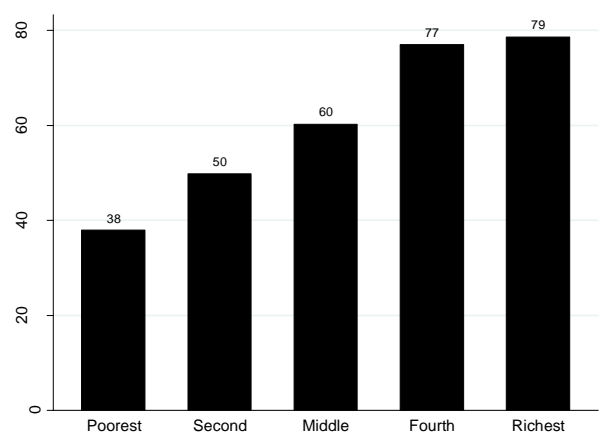

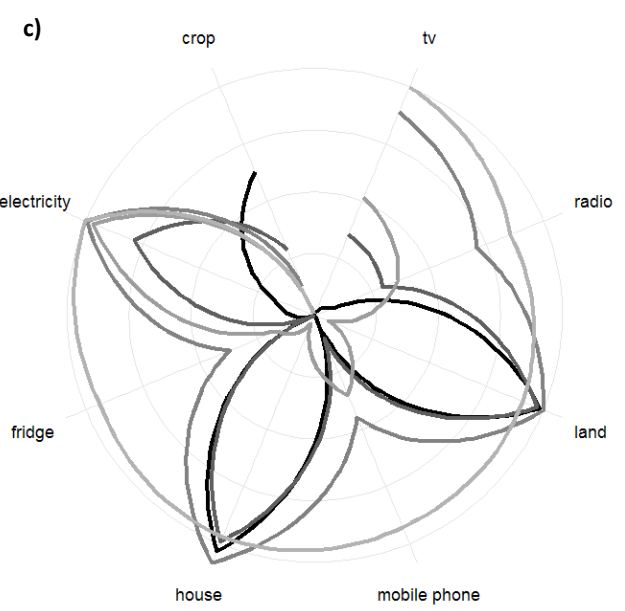

mobile phone

Figure 2.2: Results from the Discriminant Analysis of Principal Components to construct SES groupings for 2005. A) Shows the household income divided by the number of household members for each group (2014 US\$). B) Shows the monthly household expenditure divided by the household members (2014 US\$) for each group. C) Tree plot of ownership by groups from poorest (black) to richest (lightest grey).

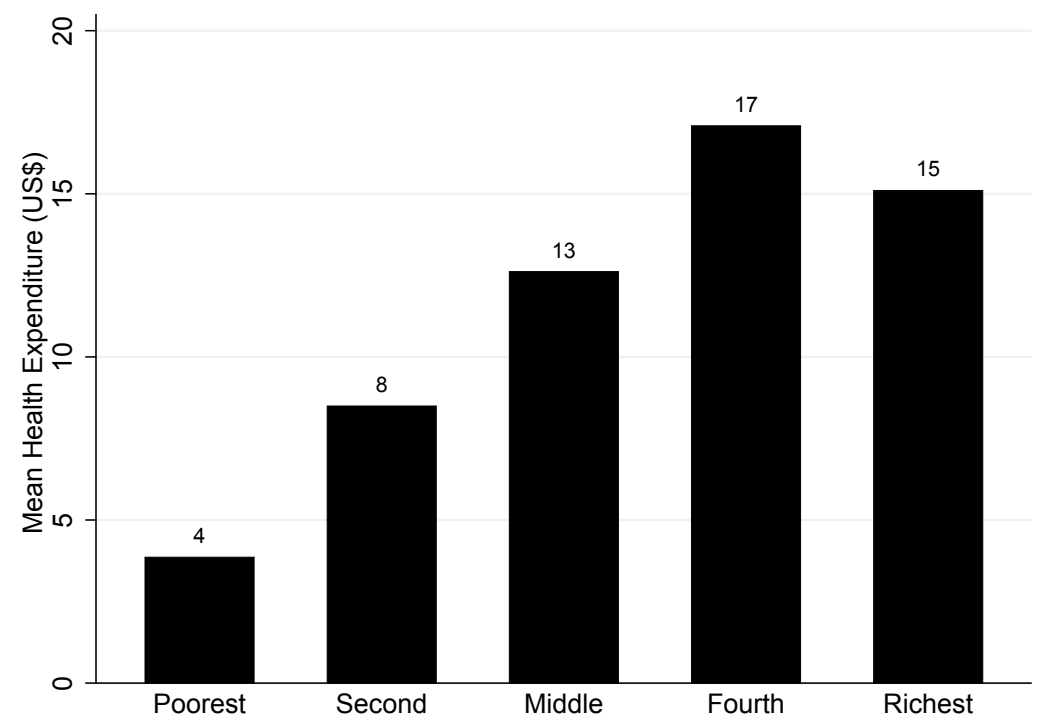

Figure 2.3: Mean household expenditure on health by SES groups. 
Table 2.2: Demographic characteristics of individuals in surveyed households.

\begin{tabular}{|c|c|c|c|c|}
\hline Characteristic & $\begin{array}{l}\text { All } \\
\text { household } \\
\text { members } \\
(\mathrm{N}=5255)\end{array}$ & $\begin{array}{l}\text { Present } \\
\text { during } \\
\text { survey } \\
(\mathrm{N}=3896)\end{array}$ & $\begin{array}{l}\text { Reported fever } \\
\text { during previous } \\
\text { month } \\
(\mathrm{N}=1631,42 \%)\end{array}$ & $\begin{array}{l}\text { Reported fever starting } \\
>2 \text { days before survey } \\
\text { and took treatment } \\
(\mathrm{N}=834,51 \%)\end{array}$ \\
\hline \multicolumn{5}{|l|}{ Age $($ years $)-\mathrm{n}(\%)$} \\
\hline $0-4$ & $870(17 \%)$ & $820(21 \%)$ & $405(25 \%)$ & $205(25 \%)$ \\
\hline $5-14$ & $1101(21 \%)$ & $824(21 \%)$ & $306(19 \%)$ & $148(18 \%)$ \\
\hline $15+$ & $3284(62 \%)$ & $2252(58 \%)$ & $920(56 \%)$ & $481(58 \%)$ \\
\hline Sex (Female $)-\mathbf{n}(\%)$ & $2409(46 \%)$ & $2019(52 \%)$ & $850(52 \%)$ & $433(52 \%)$ \\
\hline Pregnant (Yes) - n (\%) & $92(2 \%)$ & $87(2 \%)$ & $39(2 \%)$ & $23(3 \%)$ \\
\hline \multicolumn{5}{|l|}{ Place of birth - n (\%) } \\
\hline Highland Papuan & $1494(28 \%)$ & $1045(27 \%)$ & $385(24 \%)$ & $172(21 \%)$ \\
\hline Lowland Papuan & $1371(26 \%)$ & $1024(26 \%)$ & $463(28 \%)$ & $183(22 \%)$ \\
\hline Non-Papuan & $2390(45 \%)$ & $1827(47 \%)$ & $783(48 \%)$ & $479(57 \%)$ \\
\hline $\begin{array}{l}\text { Resided in lowlands for more } \\
\text { than one year? (Yes) - n (\%) }\end{array}$ & $4969(95 \%)$ & $3674(94 \%)$ & $1547(95 \%)$ & $797(96 \%)$ \\
\hline \multicolumn{5}{|l|}{ District $-\mathbf{n}(\%)$} \\
\hline Mimika Baru & $4401(84 \%)$ & $3242(83 \%)$ & $1401(86 \%)$ & $740(89 \%)$ \\
\hline Mimika Timur & $371(7 \%)$ & $286(7 \%)$ & $108(7 \%)$ & $11(1 \%)$ \\
\hline Kuala Kencana & $331(6 \%)$ & $249(6 \%)$ & $93(6 \%)$ & $65(8 \%)$ \\
\hline Tembaga Pura & $152(3 \%)$ & $119(3 \%)$ & $29(2 \%)$ & $18(2 \%)$ \\
\hline
\end{tabular}

\subsubsection{History of fevers and parasite positivity of household members}

In total $3896(74 \%)$ of individuals were present at the time of interview (Figure 2.1) of whom $48 \%$ (1877) were male, 42\% (1644) were under the age of 15 (Table 2.2), and 53\% (2069) were Papuan. In total, 1631 (42\%) of individuals from 700 (85\%) households reported having had a febrile illness in the preceding month. Significant independent risk factors for febrile illness included age, ethnicity, and poor economic status (Table 2.3).

In those who consented to blood testing, malaria slide positivity was 16\% (634/3890) of whom $46 \%$ (290) were infected with P. falciparum, 39\% (248) with P. vivax, $11 \%$ (72) with mixed infections and $4 \%(24)$ with P. malariae. Individuals in the lower three SES groups were more likely to be parasite positive than those in the top two SES groups $(\mathrm{OR}=1.33$ [95\% CI: $1.05-1.68], \mathrm{p}=0.019)$. 
Table 2.3: Logistic regression analysis of risk factors for reporting a fever in the past month $(\mathrm{N}=3896)$.

\begin{tabular}{|c|c|c|c|c|}
\hline Variable & OR $(95 \% \mathrm{CI})$ & $\mathrm{p}$ value & $\operatorname{AOR}(95 \% \mathrm{CI})$ & $\mathrm{p}$ value \\
\hline \multicolumn{5}{|l|}{ Gender } \\
\hline Male & Reference & & Reference & \\
\hline Female & $1.02(0.90-1.16)$ & 0.756 & - & - \\
\hline \multicolumn{5}{|l|}{ Age } \\
\hline$<5$ years & $1.47(1.26-1.73)$ & $<0.001$ & $1.49(1.27-1.74)$ & $<0.001$ \\
\hline$\geq 5$ years & Reference & & Reference & \\
\hline \multicolumn{5}{|l|}{ Pregnant } \\
\hline No & Reference & & Reference & \\
\hline Yes & $1.13(0.74-1.73)$ & 0.566 & - & - \\
\hline \multicolumn{5}{|l|}{ Household size } \\
\hline$\leq 7$ members & $1.16(0.98-1.38)$ & 0.091 & - & - \\
\hline$>7$ members & Reference & & Reference & \\
\hline \multicolumn{5}{|l|}{ SES groups } \\
\hline Richest & Reference & & Reference & \\
\hline Fourth & $1.47(1.11-1.95)$ & 0.007 & $1.47(1.11-1.96)$ & 0.007 \\
\hline Middle & $1.33(1.00-1.76)$ & 0.050 & $1.40(1.05-1.87)$ & 0.022 \\
\hline Second & $1.17(0.89-1.53)$ & 0.270 & $1.35(1.01-1.80)$ & 0.046 \\
\hline Poorest & $1.52(1.15-2.00)$ & 0.003 & $2.04(1.48-2.81)$ & $<0.001$ \\
\hline \multicolumn{5}{|l|}{ Ethnicity } \\
\hline Highland Papuan & Reference & & Reference & \\
\hline Lowland Papuan & $1.41(1.12-1.78)$ & 0.003 & $1.64(1.28-2.10)$ & $<0.001$ \\
\hline Non-Papuan & $1.29(1.06-1.55)$ & 0.009 & $1.59(1.26-2.01)$ & $<0.001$ \\
\hline \multicolumn{5}{|c|}{ Resided in lowlands $>1$ year? } \\
\hline No & Reference & & Reference & \\
\hline Yes & $1.19(0.86-1.66)$ & 0.292 & - & \\
\hline \multicolumn{5}{|c|}{ Family sleeps under a bednet? } \\
\hline No & Reference & & Reference & \\
\hline Yes & $1.02(0.86-1.21)$ & 0.792 & - & \\
\hline
\end{tabular}

The multiple logistic regression included variables that were significant $(\mathrm{p}<0.05)$ in the univariate logistic regression. 


\subsubsection{Treatment-seeking behaviour of household members}

Treatment-seeking behaviour was assessed in $72 \%(1176 / 1631)$ of individuals who reported fever in the preceding month (Figure 2.1). The analysis of treatment seeking was restricted to the $94 \%(1104 / 1176)$ of individuals with a fever commencing at least two days before the survey (Figure 2.1). Three-quarters $(76 \%, 834 / 1104)$ reportedly took some form of medicine. For the 270 (24\%) of participants who did not take any medication, the most common explanation offered was that the disease was not severe enough to warrant it $(169,63 \%)$.

Of those who took some form of medicine, $92 \%$ (764/834) sought treatment outside of the home at some point. Treatment seeking outside of the home was significantly more likely in those who had a febrile illness lasting two or more days (OR $=2.97[95 \%$ CI: 2.15-4.09], $\mathrm{p}<0.001)$, were in the richest two $\mathrm{SES}$ groups $(\mathrm{OR}=1.99[95 \% \mathrm{CI}$ : 1.42-2.78], $\mathrm{p}<0.001)$ and were non-Papuan $(\mathrm{OR}=2.63$ [95\% CI: 1.92-3.60], p < 0.001). Treatment seeking was not influenced by age or gender. Of those who sought treatment outside of the home, $46 \%(349 / 764)$ went to a public provider at least once; this percentage being greater in those who were female, less than 15 years old, Papuan ethnicity or from the poorest SES (Table 2.4).

The 764 individuals who sought treatment outside of the home reported a total of 894 visits to healthcare providers. Further analyses were undertaken on these 894 interactions with providers.

\subsubsection{Diagnosis of malaria at healthcare providers}

Out of the 894 visits to healthcare providers, a blood test for malaria was reportedly undertaken in 433 (48\%) of these visits (Table 2.5) of which $78 \%$ (337) were reportedly positive: half (167) P. falciparum, 47\% (159) P. vivax, 3\% (10) mixed infections and $0.3 \%$ (1) P. malariae. For 3\% (12/433) of visits, no result was reported. Children less than 14 years old were more likely to have a blood test compared to individuals over the age of 14 (OR 1.42 [95\% CI: 1.10-1.84], p = 0.007), although they were less likely 
Table 2.4: Sociodemographic characteristics associated with whether ever sought treatment at a public provider $(\mathrm{N}=764)$.

\begin{tabular}{lrrrr}
\hline \hline & \multicolumn{2}{c}{ Source of treatment $[\%(\mathrm{n})]$} & & \\
\cline { 2 - 3 } Variable & $\begin{array}{l}\text { Private provider(s) } \\
\text { only }(\mathrm{N}=415)\end{array}$ & $\begin{array}{c}\text { Public provider } \\
\text { ever }(\mathrm{N}=349)\end{array}$ & $\begin{array}{c}\text { Univariate odds } \\
\text { ratio }(95 \% \mathrm{CI})\end{array}$ & p value \\
\hline Gender & & & & \\
Male & $60 \%(219)$ & $40 \%(148)$ & Reference & \\
Female & $49 \%(196)$ & $51 \%(201)$ & $1.51(1.14-2.02)$ & $\mathbf{0 . 0 0 4}$ \\
Age group & & & & \\
15+ years & $59 \%(261)$ & $41 \%(180)$ & Reference & \\
5-15 years & $46 \%(61)$ & $54 \%(71)$ & $1.69(1.13-2.53)$ & $\mathbf{0 . 0 1 1}$ \\
$<5$ years & $49 \%(93)$ & $51 \%(98)$ & $1.53(1.06-2.21)$ & $\mathbf{0 . 0 2 4}$ \\
Ethnicity & & & & \\
Non-Papuan & $67 \%(288)$ & $33 \%(142)$ & Reference & \\
Papuan & $38 \%(127)$ & $62 \%(207)$ & $3.31(2.31-4.73)$ & $<\mathbf{0 . 0 0 1}$ \\
SES quintile & & & & \\
Highest & $76 \%(100)$ & $24 \%(32)$ & Reference & \\
Fourth & $59 \%(99)$ & $41 \%(68)$ & $2.14(1.17-3.93)$ & $\mathbf{0 . 0 1 3}$ \\
Middle & $58 \%(100)$ & $42 \%(73)$ & $2.28(1.25-4.16)$ & $\mathbf{0 . 0 0 7}$ \\
Second & $46 \%(80)$ & $54 \%(95)$ & $3.71(2.03-6.78)$ & $<\mathbf{0 . 0 0 1}$ \\
Poorest & $31 \%(36)$ & $69 \%(81)$ & $7.03(3.82-12.95)$ & $<\mathbf{0 . 0 0 1}$ \\
\hline
\end{tabular}

This is among fever patients who sought treatment outside of the home (includes inpatient and outpatient visits).

$95 \% \mathrm{CI}=95 \%$ confidence intervals.

to have a detectable parasitaemia (OR 0.30 [95\% CI: 0.18-0.51], p < 0.001).

\subsubsection{Antimalarial treatment at healthcare providers}

An antimalarial (alone or with other medicines) was reportedly prescribed during $61 \%$ (547/894) of visits: $86 \%(471 / 547)$ of which were prescribed as tablets, $5 \%(30 / 547)$ as an injection and $8 \%(46 / 547)$ as both an injection and tablets. Overall, public facilities were significantly more likely to prescribe antimalarial treatment than private providers $(\mathrm{OR}=1.41$ [95\% CI 1.07-1.86], $\mathrm{p}=0.015)$; Table 2.5, however, shows that the majority $(92 \%, 70 / 76)$ of antimalarial injections were administered by private clinics or doctors. In relation to parasitological testing prior to antimalarial prescription as per clinical 
Table 2.5: Percentage of respondents who reported receiving blood tests, antimalarials and associated costs $(\mathrm{N}=894)$.

\begin{tabular}{|c|c|c|c|c|c|c|c|c|c|c|c|}
\hline \multirow{2}{*}{ Healthcare provider } & \multirow{2}{*}{$\begin{array}{l}\text { Percentage with } \\
\text { blood test for } \\
\text { malaria n }(\%)\end{array}$} & \multirow{2}{*}{$\begin{array}{l}\text { Frequency anti- } \\
\text { malarials were } \\
\text { prescribed n }(\%)\end{array}$} & \multicolumn{3}{|c|}{ Visit costs ${ }^{\dagger}$} & \multicolumn{3}{|c|}{ Transport costs ${ }^{\dagger \dagger}$} & \multicolumn{3}{|c|}{ Total direct costs } \\
\hline & & & Mean (SD) & Median & IQR & Mean (SD) & Median & IQR & Mean (SD) & Median & IQR \\
\hline Private $(\mathrm{N}=519)$ & $148(29 \%)$ & $300(58 \%)$ & $11.52(29.86)$ & 2.80 & $0.93-8.40$ & $0.93(1.96)$ & 0.56 & $0.00-0.93$ & $12.46(30.25)$ & 3.74 & $1.49-9.99$ \\
\hline Private clinic or doctor $(\mathrm{N}=240)$ & $124(52 \%)$ & $187(78 \%)$ & $21.66(41.44)$ & 7.47 & $2.80-18.68$ & $1.53(2.73)$ & 0.84 & $0.37-1.12$ & $23.19(41.77)$ & 9.34 & $3.74-22.69$ \\
\hline Pharmacy $(\mathrm{N}=173)$ & $23(13 \%)$ & $87(50 \%)$ & $3.76(5.26)$ & 1.87 & $0.93-3.92$ & $0.58(0.39)$ & 0.56 & $0.37-0.75$ & $4.34(5.29)$ & 2.61 & $1.68-5.04$ \\
\hline Shop $(N=106)$ & $1(1 \%)$ & $26(25 \%)$ & $1.25(1.44)$ & 0.75 & $0.37-1.49$ & $0.15(0.32)$ & 0.00 & $0.00-0.19$ & $1.40(1.57)$ & 0.84 & $0.37-1.87$ \\
\hline Public $(\mathrm{N}=375)$ & $285(76 \%)$ & $247(66 \%)$ & $2.63(13.87)$ & 0.37 & $0.00-0.75$ & $0.88(1.66)$ & 0.37 & $0.00-0.93$ & $3.52(14.33)$ & 0.93 & $0.00-1.87$ \\
\hline Puskesmas $(\mathrm{N}=155)^{*}$ & $102(66 \%)$ & $94(61 \%)$ & $3.40(11.20)$ & 0.75 & $0.37-1.42$ & $0.57(0.69)$ & 0.37 & $0.00-0.75$ & $3.97(11.57)$ & 1.21 & $0.75-2.36$ \\
\hline Malaria control clinic $(\mathrm{N}=149)^{* *}$ & $123(83 \%)$ & $107(72 \%)$ & $0.00(0.00)$ & 0.00 & $0.00-0.00$ & $0.57(1.59)$ & 0.00 & $0.00-0.56$ & $0.57(1.59)$ & 0.00 & $0.00-0.56$ \\
\hline Hospital $(\mathrm{N}=71)$ & $60(85 \%)$ & $46(65 \%)$ & $6.49(26.86)$ & 0.37 & $0.37-0.93$ & $2.24(2.46)$ & 1.40 & $0.93-2.05$ & $8.72(27.41)$ & 1.87 & $1.31-2.80$ \\
\hline p value (Private versus Public) & $<0.001^{a}$ & $0.015^{b}$ & & $<0.001^{c}$ & & & $0.080^{c}$ & & & $0.001^{c}$ & \\
\hline
\end{tabular}

N $\quad$ All costs are reported in US\$.

$\dagger$ Visit costs for inpatient and outpatient visits, including costs of consultation, diagnosis, medications and any other costs directly related to the care received.

†† Transport cost includes costs for the patient and anyone who accompanied him or her.

${ }^{a} \mathrm{OR}=7.94$ [95\% CI 5.86-10.76].

${ }^{b} \mathrm{OR}=1.41$ [95\% CI 1.07-1.86].

${ }^{c}$ Mann-Whitney test.

* Government-funded primary health clinic.

** Mining company-funded clinic. 
guidelines, 61\% (336/547) of the antimalarial prescriptions followed a blood test. The corresponding figures were $87 \%(215 / 247)$ at public facilities and $40 \%(121 / 300)$ at private providers $(\mathrm{p}<0.001)$.

Only 10\% (17/177) of individuals who reported testing positive for $P$. falciparum or mixed infection reported receiving the prevailing first-line treatment of chloroquine with $\mathrm{SP}$, with the majority receiving primaquine $(129 ; 73 \%)$, chloroquine $(103 ; 58 \%)$ and/or quinine $(49 ; 28 \%)$. Those reporting a diagnosis of $P$. vivax were more likely to receive the correct first line treatment of chloroquine and primaquine [38\% (61/159); Table 2.6]. Overall, public facilities were more likely to prescribe the correct drug combinations $(\mathrm{OR}=3.77[95 \%$ CI 2.31-6.14], $\mathrm{p}<0.001)$.

In total, $69 \%$ (232/337) of respondents who reported a positive blood test for malaria, reported being prescribed primaquine. Of those who reported taking primaquine for $P$. vivax infection (alone or mixed), 63\% (69/109) reported taking less than seven days of treatment (Figure 2.4).

\subsubsection{Direct costs of treatment seeking}

The mean total direct cost to the household per visit at a private provider was US $\$ 12.46$ $(\mathrm{SD}=30.25)$ compared to $\mathrm{US} \$ 3.52(\mathrm{SD}=14.33)$ at the public sector $(\mathrm{p}<0.001)$. Table 2.5 shows the mean cost of treatment (including drugs, consultation and diagnosis) and transport (including patient and any accompanying people) by source of treatment. No significant difference in direct costs were seen for those diagnosed with $P$. falciparum as compared with P. vivax infection. The total direct costs per person according to age group are presented in Table 2.7 .

\subsubsection{Indirect costs per fever episode}

Overall, $77 \%(641 / 834)$ of those who reported taking treatment stated that at least one person had reduced his or her usual activities to care for them and 66\% (553/834) of respondents reported having at least one companion journey with them when seeking treatment. Eight percent (66/834) reported the need for at least one substitute labourer 
Table 2.6: Antimalarial treatment received according to blood test results as reported in the household survey (includes inpatient and outpatient visits).

\begin{tabular}{|c|c|c|c|c|c|c|c|c|c|}
\hline & $\begin{array}{l}\text { Private clinic } \\
\text { or doctor }\end{array}$ & Pharmacy & Shop & $\begin{array}{l}\text { All } \\
\text { private }\end{array}$ & Puskesmas $^{\dagger}$ & $\begin{array}{l}\text { Malaria } \\
\text { control clinic }^{\dagger \dagger}\end{array}$ & Hospital & $\begin{array}{c}\text { All } \\
\text { Public }\end{array}$ & Overall \\
\hline Not tested (N) & 116 & 150 & 105 & 371 & 53 & 26 & 11 & 90 & 461 \\
\hline$\%(\mathrm{n})$ antimalarials $^{a}$ & $69 \%(80)$ & $49 \%(73)$ & $25 \%(26)$ & $48 \%(179)$ & $43 \%(23)$ & $35 \%(9)$ & $0 \%(0)$ & $36 \%(32)$ & $46 \%(211)$ \\
\hline Tested negative $(\mathrm{N})$ & 16 & 5 & 1 & 22 & 28 & 21 & 13 & 62 & 84 \\
\hline$\%$ (n) antimalarials ${ }^{a}$ & $19 \%(3)$ & $0 \%(0)$ & $0 \%(0)$ & $14 \%(3)$ & $0 \%(0)$ & $5 \%(1)$ & $0 \%(0)$ & $2 \%(1)$ & $5 \%(4)$ \\
\hline P. falciparum $(\mathrm{N})$ & 57 & 11 & 0 & 68 & 29 & 50 & 20 & 99 & 167 \\
\hline$\%$ (n) antimalarials ${ }^{a b}$ & $98 \%(56)$ & $73 \%(8)$ & - & $94 \%(64)$ & $97 \%(28)$ & $100 \%(50)$ & $95 \%(19)$ & $98 \%(97)$ & $96 \%(161)$ \\
\hline$\%$ (n) chloroquine ${ }^{* b}$ & $51 \%(29)$ & $27 \%(3)$ & - & $47 \%(32)$ & $79 \%(23)$ & $88 \%(44)$ & $5 \%(1)$ & $69 \%(68)$ & $60 \%(100)$ \\
\hline$\%(\mathrm{n}) \mathrm{SP}^{* b}$ & $5(9 \%)$ & $0 \%(0)$ & - & $7 \%(5)$ & $0 \%(0)$ & $28 \%(14)$ & $0 \%(0)$ & $14 \%(14)$ & $11 \%(19)$ \\
\hline$\%$ (n) primaquine ${ }^{b}$ & $68 \%(39)$ & $27 \%(3)$ & - & $62 \%(42)$ & $83 \%(24)$ & $92 \%(46)$ & $55 \%(11)$ & $82 \%(81)$ & $74 \%(123)$ \\
\hline$\%$ (n) quinine ${ }^{b}$ & $33 \%(19)$ & $36 \%(4)$ & - & $34 \%(23)$ & $17 \%(5)$ & $6 \%(3)$ & $75 \%(15)$ & $23 \%(23)$ & $28 \%(46)$ \\
\hline$\%$ (n) antimalarial injection ${ }^{b}$ & $32 \%(18)$ & $9 \%(1)$ & - & $28 \%(19)$ & $3 \%(1)$ & $0 \%(0)$ & $10 \%(2)$ & $3 \%(3)$ & $13 \%(22)$ \\
\hline$P . \operatorname{vivax}(\mathrm{N})$ & 44 & 7 & 0 & 51 & 41 & 43 & 24 & 108 & 159 \\
\hline$\%(\mathrm{n})$ antimalarials $^{a b}$ & $95 \%(42)$ & $86 \%(6)$ & - & $94 \%(48)$ & $100 \%(41)$ & $98 \%(42)$ & $100 \%(24)$ & $99 \%(107)$ & $97 \%(155)$ \\
\hline$\%$ (n) chloroquine ${ }^{* b}$ & $34 \%(15)$ & $71 \%(5)$ & - & $39 \%(20)$ & $68 \%(28)$ & $77 \%(33)$ & $8 \%(2)$ & $58 \%(63)$ & $52 \%(83)$ \\
\hline$\%(\mathrm{n})$ primaquine $^{* b}$ & $34 \%(15)$ & $0 \%(0)$ & - & $29 \%(15)$ & $78 \%(32)$ & $81 \%(35)$ & $88 \%(21)$ & $81 \%(88)$ & $65 \%(103)$ \\
\hline$\%$ (n) quinine ${ }^{b}$ & $34 \%(15)$ & $14 \%(1)$ & - & $31 \%(16)$ & $24 \%(10)$ & $5 \%(2)$ & $88 \%(21)$ & $31 \%(33)$ & $31 \%(49)$ \\
\hline$\%$ (n) antimalarial injection ${ }^{b}$ & $11 \%(5)$ & $0 \%(0)$ & - & $10 \%(5)$ & $0 \%(0)$ & $0 \%(0)$ & $4 \%(1)$ & $1 \%(1)$ & $4 \%(6)$ \\
\hline
\end{tabular}

${ }^{\dagger}$ Government-funded primary health clinic. ${ }^{\dagger \dagger}$ Mining company-funded clinic.

${ }^{a}$ Medication categories are not mutually exclusive.

${ }^{b}$ Includes tablets and injections.

* Recommended treatment regimen. 


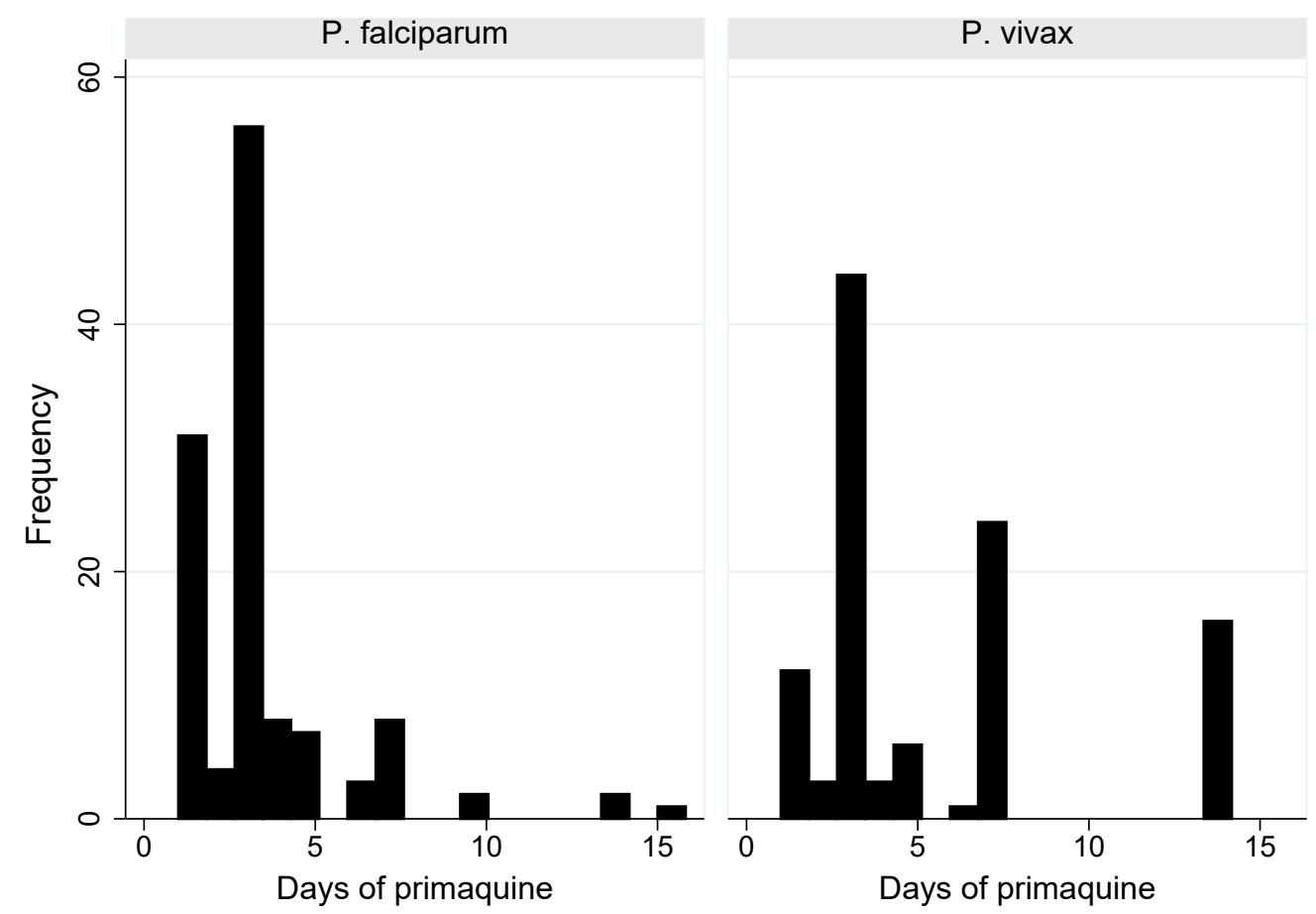

Figure 2.4: Histogram of the reported number of days of primaquine treatment for $P$. falciparum and P. vivax / mixed infections (n, \%).

to perform their usual activities while sick and this was significantly more likely in non-Papuans $(\mathrm{OR}=2.09[95 \%$ CI 1.19-3.65], $\mathrm{p}=0.010)$. Of those who reported a daily wage, the mean was US\$10.92 ( $\mathrm{SD}=8.22)$. The days lost by the individual and his/her companions, caretakers and substitute labourers are shown in Table 2.7 along with their associated costs. The total mean indirect cost per fever episode requiring treatment taking was US\$31.49 ( $\mathrm{SD}=35.92)$ for children and US\$53.52 ( $\mathrm{SD}=58.06)$ for adults.

\subsubsection{Malaria costs from healthcare facility exit survey}

A total of 358 patients were surveyed after exiting six public healthcare facilities (the hospital, two Puskesmas and three malaria control clinics). The reported diagnosis was P. falciparum in 55\% (196), P. vivax in $38 \%$ (136), mixed infection in $4 \%(12), P$. malariae in $4 \%(13)$ and $P$. ovale in $0.3 \%$ (1). The direct and indirect costs related to visits at public providers for P. falciparum and P. vivax are presented in Table 2.8. The total costs were similar between the species of diagnosis, however respondents with $P$. falciparum infection reportedly spent more money than those with $P$. vivax for the 
Table 2.7: Reported direct, indirect and total costs (including illness days) per individual taking treatment for fever over the entire fever episode $(\mathrm{N}=834)$.

\begin{tabular}{|c|c|c|c|c|c|c|c|c|c|}
\hline \multirow{2}{*}{ Category } & \multicolumn{3}{|c|}{ Child $(\mathrm{n}=353)$} & \multicolumn{3}{|c|}{ Adult $(\mathrm{n}=481)$} & \multicolumn{3}{|c|}{ Overall $(\mathrm{N}=834)$} \\
\hline & Mean (SD) & Median & IQR & Mean (SD) & Median & IQR & Mean (SD) & Median & IQR \\
\hline Total direct costs (incl. payments for all treatments) & $7.34(25.72)$ & 1.31 & $0.19-5.04$ & $10.83(28.38)$ & 1.96 & $0.41-6.16$ & $9.35(27.33)$ & 1.87 & $0.37-5.60$ \\
\hline Total indirect costs & $31.49(35.92)$ & 27.31 & $10.92-38.23$ & $53.52(58.06)$ & 38.23 & $16.39-76.46$ & $44.19(51.05)$ & 32.77 & $10.92-60.08$ \\
\hline \multicolumn{10}{|l|}{ Individual taking treatment } \\
\hline Number of days unable to perform all usual activities ${ }^{a}$ & - & - & - & $1.59(2.52)$ & 0.00 & $0.00-3.00$ & - & - & - \\
\hline Number of days unable to perform some usual activities ${ }^{a b}$ & - & - & - & $2.96(4.23)$ & 2.00 & $0.00-5.00$ & - & - & - \\
\hline Total cost to the individual due to lost wages & - & - & - & $33.47(33.95)$ & 27.31 & $10.92-43.69$ & $19.31(30.63)$ & 0.00 & $0.00-32.77$ \\
\hline \multicolumn{10}{|l|}{ All companions } \\
\hline Number of days unable to perform all usual activities & $0.17(0.57)$ & 0.00 & $0.00-0.00$ & $0.10(0.38)$ & 0.00 & $0.00-0.00$ & $0.13(0.47)$ & 0.00 & $0.00-0.00$ \\
\hline Number of days unable to perform some usual activities ${ }^{b}$ & $0.73(0.77)$ & 1.00 & $0.00-1.00$ & $0.30(0.61)$ & 0.00 & $0.00-0.00$ & $0.48(0.72)$ & 0.00 & $0.00-1.00$ \\
\hline Total cost for companions & $5.86(7.58)$ & 5.46 & $0.00-5.46$ & $2.78(5.21)$ & 0.00 & $0.00-5.46$ & $4.09(6.50)$ & 0.00 & $0.00-5.46$ \\
\hline \multicolumn{10}{|l|}{ All caretakers } \\
\hline Number of days unable to perform all usual activities & $0.31(1.73)$ & 0.00 & $0.00-0.00$ & $0.23(1.55)$ & 0.00 & $0.00-0.00$ & $0.27(1.63)$ & 0.00 & $0.00-0.00$ \\
\hline Number of days unable to perform some usual activities ${ }^{b}$ & $4.03(4.41)$ & 3.00 & $1.00-5.00$ & $2.36(4.50)$ & 0.00 & $0.00-3.00$ & $3.07(4.54)$ & 1.00 & $0.00-5.00$ \\
\hline Total cost for caretaking & $25.45(31.77)$ & 21.85 & $5.46-32.77$ & $15.42(28.92)$ & 0.00 & $0.00-21.85$ & $19.67(30.54)$ & 10.92 & $0.00-27.31$ \\
\hline \multicolumn{10}{|l|}{ All substitute labourers } \\
\hline Number of days unable to perform all usual activities & $0.00(0.00)$ & 0.00 & $0.00-0.00$ & $0.06(0.40)$ & 0.00 & $0.00-0.00$ & $0.03(0.30)$ & 0.00 & $0.00-0.00$ \\
\hline Number of days unable to perform some usual activities ${ }^{b}$ & $0.03(0.36)$ & 0.00 & $0.00-0.00$ & $0.22(1.33)$ & 0.00 & $0.00-0.00$ & $0.14(1.04)$ & 0.00 & $0.00-0.00$ \\
\hline Total cost for substitute labour & $0.17(1.95)$ & 0.00 & $0.00-0.00$ & $1.84(8.39)$ & 0.00 & $0.00-0.00$ & $1.13(6.54)$ & 0.00 & $0.00-0.00$ \\
\hline Total costs & $38.82(46.41)$ & 29.18 & $16.39-45.35$ & $64.34(74.43)$ & 40.47 & $16.39-87.39$ & $53.54(65.28)$ & 33.75 & $16.39-66.66$ \\
\hline
\end{tabular}

${ }^{a}$ Not reported by children.

${ }^{b}$ Days reported as cutting back on some activities were included in the cost calculations as half of the mean wage per day. 
healthcare visit $(\mathrm{p}=0.013)$, personal transportation to the healthcare facility $(\mathrm{p}<$ $0.001)$ and lost wages for the patient $(\mathrm{p}=0.001)$. Individuals with $P$. vivax infection spent more money on companion costs $(\mathrm{p}=0.034)$. When the analysis was restricted to children or adults, the only cost that remained statistically significant was for the healthcare visit.

Table 2.8: Mean (SD), median and IQR of patient costs in 2014 US\$ per visit to a public provider (inpatient and outpatient) by malaria diagnosis from facility exit surveys. ${ }^{a}$

\begin{tabular}{l|lll|lll|c}
\hline & \multicolumn{3}{|c|}{ P. falciparum $(\mathrm{N}=196)$} & \multicolumn{3}{c}{ P. vivax (N=136) } & \\
& Mean (SD) & Median & IQR & Mean (SD) & Median & IQR & p value \\
\hline Total direct costs & $2.69(4.67)$ & 1.42 & $0.84-2.25$ & $2.09(3.35)$ & 1.31 & $0.00-2.24$ & 0.056 \\
Visit cost & $0.90(3.08)$ & 1.42 & $0.00-0.75$ & $0.40(0.53)$ & 0.37 & $0.00-0.37$ & $\mathbf{0 . 0 1 3}$ \\
Transport cost for patient & $0.61(1.41)$ & 0.16 & $0.00-0.56$ & $0.40(1.24)$ & 0.00 & $0.00-0.28$ & $<\mathbf{0 . 0 0 1}$ \\
Transport cost for companions & $1.18(2.53)$ & 0.56 & $0.00-1.12$ & $1.28(2.61)$ & 0.28 & $0.00-1.17$ & 0.541 \\
Total indirect costs ${ }^{a}$ & $45.90(63.10)$ & 27.31 & $10.92-60.08$ & $42.41(44.98)$ & 27.31 & $10.92-54.62$ & 0.960 \\
Lost wages for patient ${ }^{b}$ & $17.23(29.73)$ & 0.00 & $0.00-27.31$ & $8.79(19.59)$ & 0.00 & $0.00-5.46$ & $\mathbf{0 . 0 0 1}$ \\
Lost wages for companions & $5.46(7.28)$ & 5.46 & $0.00-10.92$ & $6.95(7.33)$ & 5.46 & $0.00-10.92$ & $\mathbf{0 . 0 3 4}$ \\
Lost wages for caretakers & $22.04(40.55)$ & 10.92 & $0.00-30.04$ & $26.51(36.39)$ & 10.92 & $0.00-38.23$ & 0.089 \\
Lost wages for substitute labourers & $1.25(6.85)$ & 0.00 & $0.00-0.00$ & $0.16(1.87)$ & 0.00 & $0.00-0.00$ & 0.096 \\
\hline Total costs & $48.58(64.65)$ & 29.51 & $11.86-65.59$ & $44.50(46.23)$ & 28.34 & $10.92-60.73$ & 0.981 \\
\hline
\end{tabular}

${ }^{a}$ The per day wage was taken from the household survey (US\$10.93).

${ }^{b}$ For children, lost wages are recorded as no cost.

\subsection{Discussion}

This study from Papua, Indonesia, was conducted in an area with one of the greatest levels of malaria burden in the region and at a time of high levels of antimalarial drug resistance to prevailing first-line therapies. It is the first study from eastern Indonesia reporting household treatment-seeking behaviour and is one of the few studies comparing household costs of $P$. vivax malaria with P. falciparum malaria, as well as self-reported adherence to 14 day primaquine.

There were several significant findings of relevance to malaria control efforts. Firstly in relation to treatment seeking, almost a third of individuals reporting a fever in the 
last month did not seek treatment outside of the home. This figure is higher than previous reports from central Java where $88 \%$ of households reported seeking treatment or advice outside of the home for their most recent malaria illness [14]. Over half (54\%) of individuals sought treatment only in the private sector, despite the availability of free treatment in the public sector. Those who sought care in the private sector were less likely to report a diagnosis of malaria, but almost $60 \%$ still reported receiving antimalarial treatment. Fifteen percent of individuals with fever reported more than one source of treatment, a likely consequence of the initial treatment failing to improve their symptoms. These findings highlight the importance of engaging with the private sector in malaria control efforts and of the need to understand and address the reasons underlying patients preferences.

Secondly in relation to antimalarial treatment, of those patients who reported positive blood tests for malaria, few were prescribed the recommended drug regimen for the species that they reported and for patients reporting $P$. vivax infections who reportedly received primaquine, over half of participants reported taking less than three days of the recommended 14 day regimen, a duration ineffective in preventing relapse [31]. Furthermore, primaquine was not recommended for P. falciparum, but the majority of patients reported receiving it. With such a large proportion of P. falciparum patients reporting primaquine use, it seems unlikely that this is entirely due to patient recall bias in terms of the species diagnosed or the drug prescribed. Such discrepancies in antimalarial policy and actual practice have been described previously in other settings $[32,33]$. There are many possible reasons including lack of provider awareness or understanding, patient and provider preferences, perceptions and experiences with regards efficacy and side effects. Understanding and addressing the reasons for these discrepancies is clearly important, especially in the face of increasing antimalarial drug resistance.

Thirdly in relation to household costs, the household financial burden of fevers in this region was substantial and did not differ substantially by the species of malaria. The mean cost per fever episode reported in the household survey was (US\$53.54) 
and included the direct costs of the whole illness episode, including subsequent visits to treatment providers, as well as any further indirect costs of time incurred after visits to a healthcare facility. The mean cost of an episode of $P$. vivax malaria was US\$44.50, compared to US\$48.58 P. falciparum. This likely represents an underestimate of household costs as patients were at public facilities and not at the end of their malaria episode.

Over $60 \%$ of households reported a monthly income less than US\$500, indicating that a fever episode represented at least $11 \%$ of the households monthly income. This also represents nearly five hours of work using the mean reported hourly wage of US\$10.92. Individuals in poorer households were at greater risk of fevers, with additional indirect costs likely to accumulate in those who do not take effective treatment. These findings emphasize the enormous economic burden of recurrent $P$. vivax episodes on the most vulnerable communities.

Another strength of this study, is the use of Discriminant Analysis of Principal Components to create meaningful SES categories. In this setting, use of the traditional asset index would have resulted in misclassification of households into categories which would have not reflected a useful categorization of households actual status. Re-settled Papuans had been provided with house and land by the local government resulting in many of the poorer households having high levels of house and land ownership but little other disposable income. When the data were analysed using a principal components analysis, the first component of the principal components analysis only explained $19 \%$ of the variation, but the results with the Discriminant Analysis of Pricipal Components was able to explain almost all of the differences between groups using only two discriminant functions. These could then be correlated back to the original variables and extract the ones that are relevant to explain group differences. Lower SES status was a risk factor for both recent febrile illness and parasite positivity at the time of the survey. Those with lower SES status were more likely to ever seek treatment at a public facility, a likely reflection of the lower costs incurred. This, along with the higher likelihood of reporting fever in the past month by those with lower SES, highlights the heterogeneity 
of the population in relation to risk of fever and treatment-seeking behaviour.

This study has a number of limitations. Firstly, much time has elapsed since the collection of the data and the presentation of the results. While it is possible that this has resulted in some of the loss of some of the finer details of the study, the survey design and results published by Karyana et al. in 2008 provide a record of the substantive details of the survey [8]. Many of the older male household members were not present at the time of the survey and therefore not included in the analysis. In this region it is often the adult males who are at higher risk of malaria and so it is possible that we underestimated the true burden of fever episodes. Furthermore, males were more likely than females to seek treatment at private providers, which would have impacted the overall results with regards to treatment-seeking behaviour and costs [8]. Another group excluded from the survey were temporary migrants who had moved into the area within last six months and did not plan to stay longer than six months. Depending on where they moved from, these individuals might be more susceptible to malaria than the general population and more likely to cause malaria outbreaks. The survey was also undertaken in the four most accessible and populous subdistricts whose population may not be representative of those in the more remote subdistricts where access to healthcare is more difficult therefore results may slightly overestimate the proportion seeking treatment outside the home.

A common issue with all surveys aimed at describing treatment-seeking behaviour and case management for malaria, is the unknown malaria status of the individuals who reported having a fever in the past month. Including all fevers in the previous month, enables the potential capture of entire fever episodes; however, they may also result in significant recall bias due to the length of time between the episode and the survey. In order to triangulate these results, healthcare facility exit surveys were undertaken in which patients were interviewed immediately after their visit, thus minimising the potential for bias and enabling an examination of costs specific to the type of malaria diagnosed. Any treatment or indirect costs occurring after the healthcare visit, however, would not be captured by this survey and thus potentially impact its cost estimates. 
This study was undertaken at a time of very high treatment failure to the recommended first-line antimalarials [11]. The poor efficacy of available treatments may partly explain the high rates of reported fever in the preceding month and why a third of respondents did not access treatment outside of the home.

\subsubsection{Conclusions}

In summary these findings highlight a high level of reported febrile illness, which is most apparent in young children and those from poor households. Despite the provision of free treatment in public health facilities, treatment seeking in the private sector was higher than expected, with considerably higher costs. The household costs of $P$. vivax were similar to $P$. falciparum, and in view of the relapsing and recurrent nature of malaria in this location, the financial burden of both infections is likely to be considerable. Antimalarial programmes implementing policy change of first-line treatment will need to engage both public and private sectors if early diagnosis and highly effective treatment is to impact on the whole community. 


\subsection{Declarations}

\subsubsection{Acknowledgements}

I am grateful to Lembaga Pengembangan Masyarakat Amungme Kamoro for ongoing support. I thank Mauritz Okeseray, Ferryanto Chalfein, Budi Prasetyorini, Peter Ebsworth and Michael Bangs for their support and technical assistance. I am also grateful to Morrison Bethea, PT Freeport Indonesia and International SOS for their ongoing support.

\subsubsection{Ethics approval}

This study was approved by the Ethics Committee of the National Institute of Health research and Development (NIHRD), Indonesian Ministry of Health and the Human Research Ethics Committee of NT Department of Health \& Community Services (KS.02.01.2.1.2684) and Menzies School of Health Research, Darwin, Australia (HREC 04/47). Written informed consent was obtained from all adult participants and parents of enrolled children. 


\section{References for Chapter 2}

[1] World Health Organisation. Guidelines for the Treatment of Malaria. 2015;

[2] McCombie SC. Treatment seeking for malaria: a review of recent research. Soc Sci Med. 1996;43(6):933-45.

[3] Williams HA, Jones CO. A critical review of behavioral issues related to malaria control in sub- Saharan Africa: what contributions have social scientists made? Soc Sci Med. 2004;59(3):501-23. doi:10.1016/j.socscimed.2003.11.010.

[4] Population Services International and ACTwatch. Malaria Market Trends in Sub-Saharan Africa: 2009-2014; 2015.

[5] Littrell M, Gatakaa H, Evance I, Poyer S, Njogu J, Solomon T, et al. Monitoring fever treatment behaviour and equitable access to effective medicines in the context of initiatives to improve ACT access: baseline results and implications for programming in six African countries. Malar J. 2011;10:327. doi:10.1186/1475-2875-10-327.

[6] ACTwatch Group and PSI/Cambodia. Kingdom of Cambodia Household Survey Report, 2011; 2011. Available from: www.actwatch.info.

[7] ACTwatch Group and PSI/Myanmar. The Republic of the Union of Myanmar Household Survey: Baseline, 2012. Population Services International; 2012. Available from: www. actwatch.info.

[8] Karyana M, Burdarm L, Yeung S, Kenangalem E, Wariker N, Maristela R, et al. Malaria morbidity in Papua Indonesia, an area with multidrug resistant Plasmodium vivax and Plasmodium falciparum. Malar J. 2008;7:148. doi:10.1186/1475-2875-7-148.

[9] Langford S, Douglas NM, Lampah DA, Simpson JA, Kenangalem E, Sugiarto P, et al. Plasmodium malariae Infection Associated with a High Burden of Anemia: 
A Hospital-Based Surveillance Study. PLoS Negl Trop Dis. 2015;9(12):e0004195. doi:10.1371/journal.pntd.0004195.

[10] Elyazar IR, Hay SI, Baird JK. Malaria distribution, prevalence, drug resistance and control in Indonesia. Adv Parasitol. 2011;74:41-175.

doi:10.1016/b978-0-12-385897-9.00002-1.

[11] Ratcliff A, Siswantoro H, Kenangalem E, Wuwung M, Brockman A, Edstein MD, et al. Therapeutic response of multidrug-resistant Plasmodium falciparum and P. vivax to chloroquine and sulfadoxine-pyrimethamine in southern Papua, Indonesia. Trans R Soc Trop Med Hyg. 2007;101(4):351-9.

[12] Ratcliff A, Siswantoro H, Kenangalem E, Maristela R, Wuwung RM, Laihad F, et al. Two fixed-dose artemisinin combinations for drug-resistant falciparum and vivax malaria in Papua, Indonesia: an open-label randomised comparison. Lancet. 2007;369(9563):757-65. doi:10.1016/s0140-6736(07)60160-3.

[13] Hasugian AR, Purba HL, Kenangalem E, Wuwung RM, Ebsworth EP, Maristela R, et al. Dihydroartemisinin-piperaquine versus artesunate-amodiaquine: superior efficacy and posttreatment prophylaxis against multidrug-resistant Plasmodium falciparum and Plasmodium vivax malaria. Clin Infect Dis. 2007;44(8):1067-74. doi:10.1086/512677.

[14] Sanjana P, Barcus MJ, Bangs MJ, Ompusunggu S, Elyazar I, Marwoto H, et al. Survey of community knowledge, attitudes, and practices during a malaria epidemic in central Java, Indonesia. Am J Trop Med Hyg. 2006;75(5):783-9.

[15] Badan Pusat Statistik-Statistics Indonesia (BPS) and ORC Macro. Indonesia Demographic and Health Survey 2002-2003. BPS and ORC Macro; 2003.

[16] Lalchhuanawma R, Murhekar MV. Health-seeking behaviour for febrile illness in malaria-endemic Kolasib district, Mizoram, India. Int Health. 2012;4(4):314-9. doi:10.1016/j.inhe.2012.06.003. 
[17] Xu JW, Xu QZ, Liu H, Zeng YR. Malaria treatment-seeking behaviour and related factors of Wa ethnic minority in Myanmar: a cross-sectional study. Malar J. 2012;11:417. doi:10.1186/1475-2875-11-417.

[18] Kirkby K, Galappaththy GN, Kurinczuk JJ, Rajapakse S, Fernando SD. Knowledge, attitudes and practices relevant to malaria elimination amongst resettled populations in a post-conflict district of northern Sri Lanka. Trans R Soc Trop Med Hyg. 2013;107(2):110-8. doi:10.1093/trstmh/trs015.

[19] Mazumdar S. Prevalence, risk factors and treatment-seeking behaviour for malaria: the results of a case study from the Terai region of West Bengal, India. Ann Trop Med Parasitol. 2011;105(3):197-208. doi:10.1179/136485911x12987676649548.

[20] Davy CP, Sicuri E, Ome M, Lawrence-Wood E, Siba P, Warvi G, et al. Seeking treatment for symptomatic malaria in Papua New Guinea. Malar J. 2010;9:268. doi:10.1186/1475-2875-9-268.

[21] Yeung S, Van Damme W, Socheat D, White NJ, Mills A. Access to artemisinin combination therapy for malaria in remote areas of Cambodia. Malar J. 2008;7:96. doi:10.1186/1475-2875-7-96.

[22] Chaturvedi HK, Mahanta J, Pandey A. Treatment-seeking for febrile illness in north-east India: an epidemiological study in the malaria endemic zone. Malar J. 2009;8:301. doi:10.1186/1475-2875-8-301.

[23] ORC Macro. Guidelines for Sampling for the Malaria Indicator Survey; 2005.

[24] StataCorp. Stata Statistical Software: Release 14. 2015;.

[25] R Core Team. R: A Language and Environment for Statistical Computing. 2015; Available from: https://www.R-project.org/.

[26] Jombart T, Devillard S, Balloux F. Discriminant analysis of principal components: a new method for the analysis of genetically structured populations. BMC Genet. 2010;11:94. doi:10.1186/1471-2156-11-94. 
[27] Vyas S, Kumaranayake L. Constructing socio-economic status indices: how to use principal components analysis. Health Policy Plan. 2006;21(6):459-68. doi:10.1093/heapol/czl029.

[28] OANDA. Currency Converter: Historical Exchance Rates for 2005;Available from: http://www. oanda.com/currency/historical-rates/(Accessed: 3 March 2015).

[29] The World Bank. Inflation, consumer prices (annual \%). 2015;Available from: http://data.worldbank.org/indicator/FP.CPI.TOTL.ZG(Accessed: 4 August 2015).

[30] Conteh L, Walker D. Cost and unit cost calculations using step-down accounting. Health Policy Plan. 2004;19(2):127-35.

[31] Price RN, Douglas NM, Anstey NM, von Seidlein L. Plasmodium vivax treatments: what are we looking for? Curr Opin Infect Dis. 2011;24(6):578-85. doi:10.1097/QCO.0b013e32834c61e3.

[32] Williams HA, Durrheim D, Shretta R. The process of changing national malaria treatment policy: lessons from country-level studies. Health Policy Plan. 2004;19(6):356-70. doi:10.1093/heapol/czh051.

[33] Goodman C, Brieger W, Unwin A, Mills A, Meek S, Greer G. Medicine sellers and malaria treatment in sub-Saharan Africa: what do they do and how can their practice be improved? Am J Trop Med Hyg. 2007;77(6 Suppl):203-18. 


\title{
Chapter 3
}

\section{Treatment-seeking behaviour after}

\author{
the implementation of a unified
}

policy of dihydroartemisinin-

\author{
piperaquine for the treatment of
}

uncomplicated malaria in Papua,

\section{Indonesia}

This chapter has been published as Devine A, Kenangalem E, Burdarm FH, Anstey NM, Poespoprodjo JR, Price RN, Yeung S. Treatment-seeking behavior after the implementation of a unified policy of dihydroartemisin-piperaquine for the treatment of uncomplicated malaria in Papua, Indonesia. Am J Trop Med Hyg. 2018;98(2):543550. https://doi.org/10.4269/ajtmh.17-0680. PMID: 29280424. 


\subsection{Introduction}

In Indonesia the greatest prevalence of malaria is in the eastern provinces of Nusa Tenggara Timur and Papua [1]. Great progress has been made in reducing the burden of malaria with the majority $(74 \%)$ of the Indonesian population residing in malaria-free areas. However, $12 \%$ of the population still reside in areas with a malaria incidence greater than one case per 1000 per year [2], and these individuals are at increased risk of malaria associated anaemia [3, 4]. Until 2006, the first line policy was chloroquine plus sulfadoxine-pyrimethamine for uncomplicated P. falciparum malaria, and chloroquine plus primaquine (total dose $3.5 \mathrm{mg} / \mathrm{kg}$ over 14 days) for radical cure for P. vivax with the exception of infants less than one year of age or below and pregnant or lactating women for whom chloroquine alone was recommended [5] (Table 3.1). In 2005 clinical trials in Papua, Indonesia highlighted very high levels of antimalarial drug resistance to the recommended treatment regimens with recrudescent $P$. falciparum and recurrent $P$. vivax infections exceeding $40 \%$ by day 28 following treatment $[5,6,7]$. Dihydroartemisinin-piperaquine (DHP) was shown to be highly effective against both species [8, 9]. In response, national policy was changed in March 2006 to the fixed dose ACT of DHP for uncomplicated malaria due to any species of malaria $[8,9]$. The recommendation of single dose of primaquine for $P$. falciparum remained while the dose of primaquine recommended for $P$. vivax was increased to a total of $7 \mathrm{mg} / \mathrm{kg}$ over 14 days. The policy also stipulated that antimalarial treatment should only be given after laboratory confirmation, which was not a recommendation before 2006 .

Prior to the change in policy, a cross-sectional household survey was undertaken in 2005 , which documented that $42 \%$ of individuals reported having a febrile illness in the preceding month, for which they sought treatment multiple times, often at different healthcare facilities (Chapter 2). Only $46 \%$ of these individuals reported seeking treatment at a public provider, despite provision of treatment for free in the public sector. The majority of households reported a monthly income less than US\$500, hence each individuals fever episode resulted a cost of at least $11 \%$ of the household income 
Table 3.1: Recommended drug treatments for malaria.

\begin{tabular}{|c|c|c|}
\hline Malaria & Before March 2006 & After March 2006 \\
\hline \multicolumn{3}{|l|}{ P. vivax } \\
\hline Uncomplicated & Chloroquine with 14 day PQ $(3.5 \mathrm{mg} / \mathrm{kg})$ & DHP with 14 day PQ $(7 \mathrm{mg} / \mathrm{kg})$ \\
\hline Second line & Quinine and Doxycycline/Clindamycin & Quinine and Doxycycline/Clindamycin \\
\hline Severe & Quinine & IV Artesunate \\
\hline \multicolumn{3}{|l|}{ P. falicparum } \\
\hline Uncomplicated & Chloroquine with SP \& single-dose PQ & DHP with single dose PQ \\
\hline Second line & Quinine and Doxycycline/Clindamycin & Quinine and Doxycycline/Clindamycin \\
\hline Severe & Quinine & IV Artesunate \\
\hline
\end{tabular}

(Chapter 2). Following policy change, DHP was provided free of charge at public health facilities but was also available at a cost in the private sector.

Changes in antimalarial policy can potentially impact on the overall burden and transmission of malaria, not only through the direct impact of switching to a more effective treatment but also through increasing the communitys trust in the public health care system and the subsequent shifts in treatment-seeking behaviour. A second household survey was conducted in 2013 to investigate the changes in treatment-seeking behaviour after the policy change. The aim of this study was to describe the changes in treatment-seeking behaviour for fever and burden of disease in Papua following policy change to ACT for all species of malaria.

\subsection{Methods}

Household surveys were conducted in Mimika Regency, southern Papua, Indonesia in 2005 and 2013. The methods in 2013 were based on the 2005 survey which has been reported previously (Chapter 2). Minor differences between the questionnaires included the collection of household income as a range rather than a specific estimate and information on bed net use by individuals rather than ownership by the household.

The demographics, malaria transmission, and health services in this region have been described previously $[4,10,3]$. In brief, the district covers an area of $21,522 \mathrm{~km}^{2}$ with a population of approximately 120,457 in 2005 expanding to 202,350 in 2013. Most 
of the population resides in 12 districts, incorporating Timika town and the surrounding villages. The area is covered with thick rain forest with both coastal and mountainous areas, inhabited by a variety of ethnic groups which are broadly categorized as lowland Papuans, highland Papuans or non-Papuans. Malaria transmission occurs throughout the year and is restricted to the lowland area where most of the population lives. There are three mosquito vectors: Anopheles koliensis, Anopheles farauti and Anopheles punctulatus.

Healthcare provision is provided free of charge at public providers, which includes one hospital, 12 Government-funded Primary Health Clinics (Puskesmas), and 10 malaria control clinics provided by a local mining company. In 2008 a second hospital was opened, providing care to approximately 100,000 patients per year. Antimalarials can also be purchased in the private sector, including clinics, pharmacies and drug stores [11]. Diagnostic tests for malaria are available in the private sector but usually not in drug stores.

The household sampling strategy was the same for both surveys with 800 households sampled using a three-stage cluster sampling procedure to identify 32 clusters of 25 households [12] \& (Chapter 2). Firstly the four most populous sub-districts (Mimika Baru, Kuala Kencana, Iwaka, Kwamki Narama, and Wania) were chosen from the 12 sub-districts based on their accessibility by road from the main town of Timika and because they included the majority (80\%) of the districts population. The 2013 survey took place in similar geographic areas as the 2005 survey but the names of the subdistricts were different in 2013 due to the renaming of subdistricts after the 2005 survey. Secondly, the number of clusters in each sub-district was apportioned according to their relative populations. The houses were then assigned numbers according to their geographical location and clusters of 25 houses were identified.

The 2013 survey was conducted in the months of April through July while the 2005 survey was conducted between the months of July and December. Household members were defined as all individuals living under one roof and who ate from one kitchen. If a selected household was empty, the interviewer returned the next day. If still unoccupied, 
the next occupied household was sampled instead. As the recall period for the survey was fever in the previous month, temporary migrants who had moved into the area within the preceding month were excluded from the survey.

\subsubsection{Data collection}

The head of the household or another suitable adult was asked questions about the household and its members. Those members who were present at the time of the survey were asked specific details about any recent febrile illness and underwent a brief examination. Capillary or venous blood samples were collected for blood film examination. Symptomatic illness was defined as a history of fever in the past 24 hours or an axillary temperature greater than or equal to $37.5^{\circ} \mathrm{C}$ when examined. Nearly all surveys were conducted in Bahasa Indonesian and the remaining in a local Papuan language. Individuals reporting a history of fever in the preceding 30 days were asked to complete a separate module on treatment seeking if they were present. This module comprised of questions on all of the places where they had sought treatment, whether they had received a blood test, whether they were prescribed antimalarials or other medicines, and any associated costs to the households.

\subsubsection{Data analysis}

Data were analyzed using STATA statistical software (version 14.2) [13] and R (version 3.2.3) [14]. A Discriminant Analysis of Principal Components was used to categorize households by SES by data on reported ownership of assets [15] (Chapter 2). To ensure that sufficient time had elapsed to capture treatment-seeking behavior, those individuals reporting fever starting within the preceding two days were excluded from the analysis of treatment seeking. For the use of diagnostics, laboratory tests, and antimalarial prescriptions, each patient-provider interaction ("visit") was analyzed for public and private healthcare providers.

Frequencies and percentages were used for the descriptive data. Percentages were compared using the chi-square test $\left(\mathrm{X}^{2}\right)$. Differences in outcome distributions were 
evaluated with the Mann-Whitney test. Continuous variables were compared using Spearman's rank for correlation. When less than five observations were seen in a category, categorical outcomes were compared using Fischer's Exact test. Simple logistic regression adjusted for clustering by households was used to calculate ORs for comparisons within the 2013 survey. For the analysis of reported fever in the past month, significant risk factors $(\mathrm{P}<0.05)$ in the univariate analysis were included in a multiple logistic regression model and significant interactions between age, ethnicity and significant variables were reported. The 2005 and 2013 surveys were compared using AORs for age $(0-4,5-14,15+)$, gender, household SES and ethnicity (non-Papuan, highland Papuan, lowland Papuan). The models were adjusted for clustering by household.

Costs were gathered in Indonesian rupiah, converted into US\$ using the average exchange rates $[16,17]$ and then revised to the 2014 equivalent using the consumer price index for Indonesia [18]. The mean cost and SD per fever episode are reported. Direct costs included transportation for the patient and carer and treatment, which included consultations, diagnosis, medication, overnight stay and any other payments. Indirect costs included the amount of time that usual activities were reduced for the individual and any companions, caretakers, or substitute laborers. This time was valued using the mean reported wage for all.

\section{$3.3 \quad$ Results}

\subsubsection{Household characteristics}

A total of 800 households with 4,010 individuals were included in the 2013 survey (Figure 3.1). The baseline characteristics for both the 2005 and 2013 surveys are provided in (Table 3.2). In 2013 the median household size was five individuals (IQR $=4-6)$ with a maximum of 13 in one house. The median duration that heads of households reported residing at their current location was 13 years (IQR $=6-20$ ), with $23(3 \%)$ households reporting a move into that location within the previous year. 
Table 3.2: Demographic characteristics of all household members and individuals present during surveys in 2005 and 2013.

\begin{tabular}{|c|c|c|c|c|}
\hline \multirow[b]{2}{*}{ Characteristic - n (\%) } & \multicolumn{2}{|c|}{2005} & \multicolumn{2}{|c|}{2013} \\
\hline & $\begin{array}{l}\text { All household } \\
\text { members } \\
(\mathrm{N}=5255)\end{array}$ & $\begin{array}{l}\text { Present during } \\
\text { survey } \\
(\mathrm{N}=3896) \\
\end{array}$ & $\begin{array}{l}\text { All household } \\
\text { members } \\
(\mathrm{N}=4010)\end{array}$ & $\begin{array}{l}\text { Present during } \\
\text { survey } \\
(\mathrm{N}=2830)\end{array}$ \\
\hline \multicolumn{5}{|l|}{ Age (years) } \\
\hline $0-4$ & $870(17 \%)$ & $820(21 \%)$ & $629(16 \%)$ & $559(20 \%)$ \\
\hline $5-14$ & $1101(21 \%)$ & $824(21 \%)$ & $970(24 \%)$ & $666(24 \%)$ \\
\hline $15+$ & $3284(62 \%)$ & $2252(58 \%)$ & $2,404(60 \%)$ & $1604(57 \%)$ \\
\hline Not reported & - & - & $7(0.2 \%)$ & $1(0.04 \%)$ \\
\hline Sex (Female) & $2409(46 \%)$ & $2019(52 \%)$ & $2035(51 \%)$ & $1668(59 \%)$ \\
\hline Pregnant (Yes) & $92(2 \%)$ & $87(2 \%)$ & $-{ }^{*}$ & $45 / 2825(2 \%)$ \\
\hline \multicolumn{5}{|l|}{ Place of birth } \\
\hline Highland Papuan & $1494(28 \%)$ & $1045(27 \%)$ & $733(18 \%)$ & $539(19 \%)$ \\
\hline Lowland Papuan & $1371(26 \%)$ & $1024(26 \%)$ & $1039(26 \%)$ & $751(27 \%)$ \\
\hline Non-Papuan & $2390(45 \%)$ & $1827(47 \%)$ & $2238(56 \%)$ & $1,540(54 \%)$ \\
\hline Resided in lowlands for more than 1 year? (Yes) & $4969(95 \%)$ & $3674(94 \%)$ & $3862(96 \%)$ & $2711(96 \%)$ \\
\hline \multicolumn{5}{|l|}{ Subdistrict } \\
\hline Banti & $152(3 \%)$ & $119(3 \%)$ & $0(0 \%)$ & $0(0 \%)$ \\
\hline Harapan \& Kwamki Lama & $901(17 \%)$ & $576(15 \%)$ & $111(3 \%)$ & $80(3 \%)$ \\
\hline Inauga & $521(10 \%)$ & $405(10 \%)$ & $383(10 \%)$ & $291(10 \%)$ \\
\hline Kamoro Jaya & $354(7 \%)$ & $273(7 \%)$ & $369(9 \%)$ & $257(9 \%)$ \\
\hline Kadun Jaya, Kaugapu \& Pigapu & $371(7 \%)$ & $286(7 \%)$ & $267(7 \%)$ & $210(7 \%)$ \\
\hline Limau Asri, Iwaka, Mulia Kencana \& Naena Muktipura & $136(3 \%)$ & $112(3 \%)$ & $506(13 \%)$ & $334(12 \%)$ \\
\hline Koperapoka & $957(18 \%)$ & $729(19 \%)$ & $753(19 \%)$ & $521(18 \%)$ \\
\hline Timika Jaya, Kwamki \& Kwamki Baru & $1,407(27 \%)$ & $1,046(27 \%)$ & $989(25 \%)$ & $696(25 \%)$ \\
\hline Wonosari Jaya \& Nawaripi & $122(2 \%)$ & $98(3 \%)$ & $374(9 \%)$ & $267(9 \%)$ \\
\hline Karang Senang \& Bhintuka & $334(6 \%)$ & $252(6 \%)$ & $258(6 \%)$ & $174(6 \%)$ \\
\hline
\end{tabular}

$*$ Not reported.

In 2005 household size was six individuals (IQR $=4-8)$ with a median duration of nine years $(\mathrm{IQR}=4-15)$. Of the 781 households in which monthly household income was reported, $52 \%$ (403) estimated US\$560 or less. The households were categorized into five SES groups, with number of households in each group ranging from 91 to 296. Whilst poorer households were more likely to grow their own crops and drink rain water, richer households were more likely to own motorcycles and large electrical items and drink bought water (Figure 3.2). The poorest SES group was composed of a greater percentage of Highland Papuans $(32 \%, 47 / 145)$ compared to $19 \%(35 / 189)$ of Lowland Papuans and 3\% (15/465) of non-Papuans $(\mathrm{p}<0.001)$. The estimated household general health expenditure was correlated with the number of individuals reporting fever in a household $(\rho=0.488, \mathrm{p}<0.001)$. 


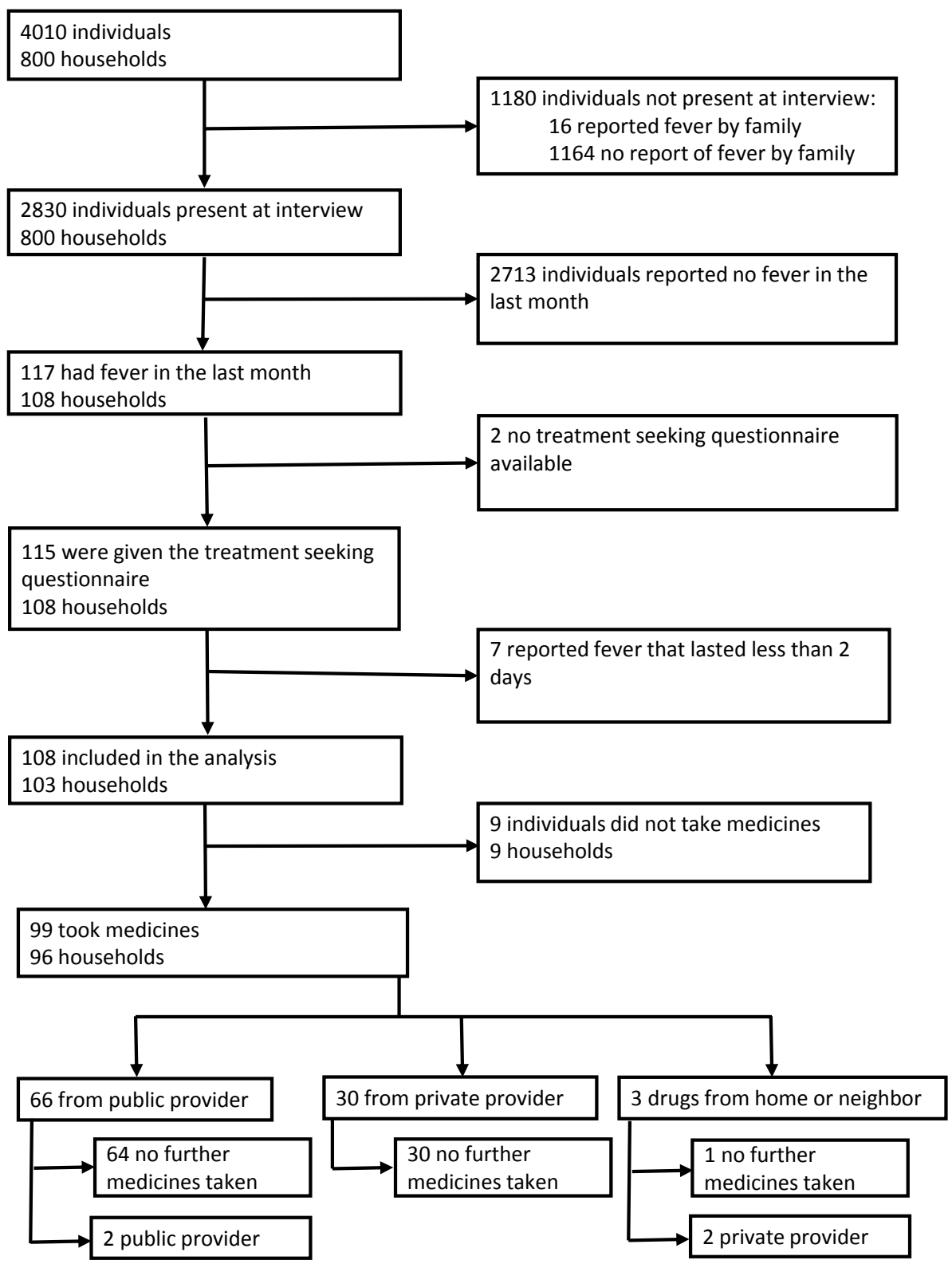

Figure 3.1: Flow diagram of household members, interviews, initial location of treatment taking, and whether took a second treatment in 2013. 


\section{bought water tv}

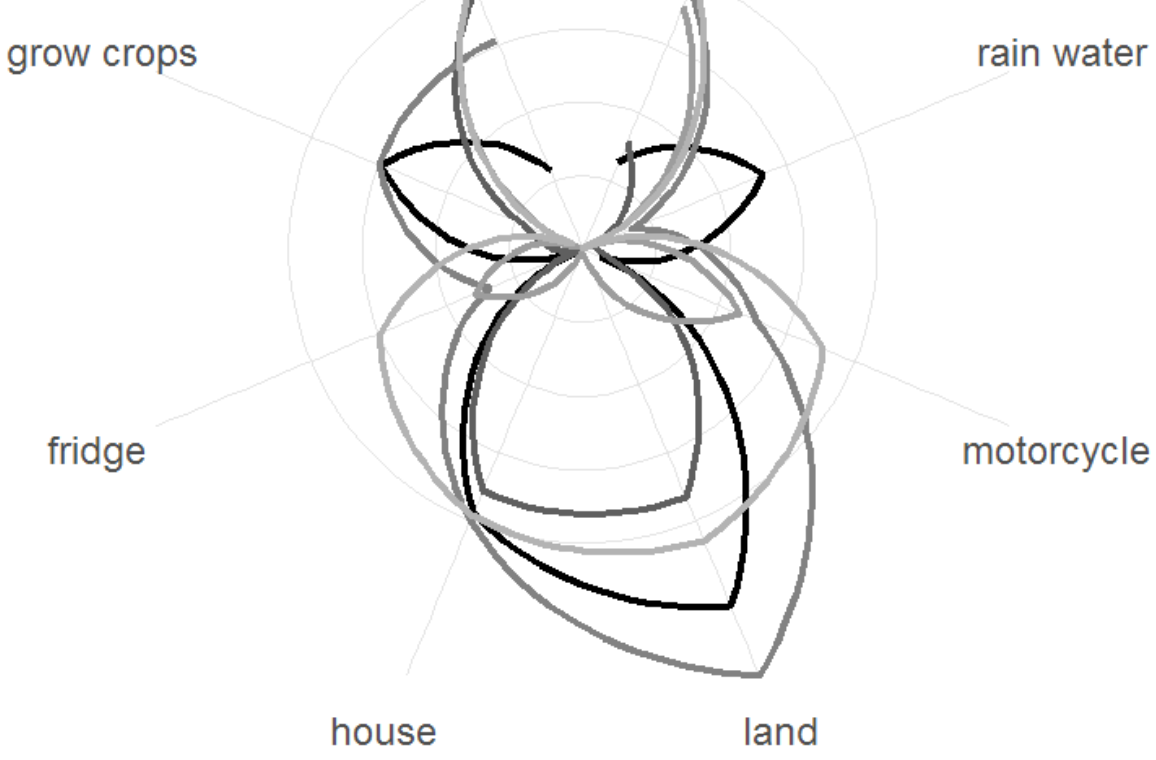

Figure 3.2: Results from the Discriminant Analysis of Principal Components to construct SES groupings for 2013. Tree plot of ownership by groups from poorest (black) to richest (lightest grey).

\subsubsection{History of fever and parasite positivity of household members}

Of the 2,830 (71\%) individuals present at the time of the interview in $2013,41 \%$ (1162) were male, $43 \%$ (1225) were under the age of 15, and $46 \%$ (1290) were Papuan (Table 3.2). Overall, 4\% (117/2830) of individuals reported having had a febrile illness in the preceding month compared with $42 \%(1631 / 3896)$ in 2005 (AOR $=0.06$ [95\% CI: 0.05-0.08], p < 0.001; (Table 3.3)). Non-Papuan participants were at a significantly higher risk for having had a febrile illness compared with Highland Papuans (AOR = 1.84 [95\% CI: 1.05-3.26], $\mathrm{p}=0.035$; Table 3.4).

Overall 12\% (348/2795) of individuals who consented to blood testing in 2013 were parasitaemic compared to $16 \%(634 / 3890)$ in $2005(\mathrm{AOR}=0.83$ [95\% CI: 0.69-0.99], p $=0.038)$. The prevalence of parasitaemia which decreased from 5\% (200/3890) in 2005 to $1 \%(21 / 2795)$ in $2013(\mathrm{AOR}=0.16$ [95\% CI: 0.10-0.26], $\mathrm{p}<0.001)$, whereas the 
prevalence of asymptomatic parasitaemia was similar in both surveys: 11\% (434/3890) in 2005 and $12 \%(327 / 2795)$ in $2013(\mathrm{AOR}=1.20$ [95\% CI: 0.98-1.45], $\mathrm{p}=0.073)$. Individuals reporting a febrile illness in the past month were significantly more likely to be parasitaemic in the 2005 survey $(\mathrm{OR}=3.81$ [95\% CI: 3.10-4.68], $\mathrm{p}<0.001)$; however, this was not significant in the 2013 survey $(\mathrm{OR}=1.53$ [95\% CI: 0.89-2.61], p $=0.123)$. The prevalence of parasitaemia in each subdistrict is presented in Table 3.5.

\subsubsection{Treatment-seeking behaviour}

In 2013 treatment-seeking behaviour was assessed in 98\% (115/117) of participants reporting a fever in the preceding month, and in $94 \%(108 / 115)$ of these individuals the fever commenced at least two days prior to the survey (Figure 3.1). Overall individuals were more likely to take some form of medicine for their fever in 2013 with $92 \%$ (99/108) reporting taking medication, compared to $76 \%(834 / 1104)$ of those whose fever commenced at least two days prior to the survey in 2005 (AOR $=2.50[95 \% \mathrm{CI}$ : $1.23-5.06], \mathrm{p}=0.011$ ). Of the 99 patients reporting taking medication, only $1 \%$ (1) did not seek treatment outside of the home, compared to $8 \%(70 / 834)$ in $2005(\mathrm{p}=0.004)$. In $201367 \%$ (66/98) of individuals seeking treatment outside of the home attended a public provider at least once compared to $46 \%(349 / 764)$ in 2005 (AOR $=4.30[95 \%$ CI: 2.54-7.28], $\mathrm{p}<0.001)$. Furthermore, individuals in the three lower SES groups were more likely to seek treatment at a public provider at least once compared to those in the top two SES groups ( $\mathrm{OR}=6.10$ [95\% CI: 2.0518.11], $\mathrm{p}=0.001)$; this was also apparent in $2005(\mathrm{OR}=2.29$ [95\% CI 1.59-3.32], $\mathrm{p}<0.001)$. While the percentage seeking treatment at at the old hospital remained constant at $8 \%(\mathrm{p}=0.961)$, the percentage of visits at any hospital increased significantly from $8 \%$ (71/894) in 2005 to $18 \%(18 / 100)$ in $2013(\mathrm{p}=0.001)$.

\subsubsection{Diagnosis of malaria at healthcare providers}

Of the 100 visits to healthcare providers in 2013, 95 (95\%) involved a blood test for malaria compared to $48 \%(433 / 894)$ in 2005 (AOR $=24.42$ [95\% CI: 9.87-65.43], p < 
Table 3.3: Fever reporting in those who were present during the survey in 2005 and 2013.

\begin{tabular}{|c|c|c|c|c|}
\hline \multirow[b]{2}{*}{ Characteristic $-\mathrm{n}(\%)$} & \multicolumn{2}{|c|}{2005} & \multicolumn{2}{|c|}{2013} \\
\hline & $\begin{array}{l}\text { Reported fever during } \\
\text { previous month } \\
(\mathrm{N}=1631 / 3896,42 \%)\end{array}$ & $\begin{array}{l}\text { Reported fever starting } \\
>2 \text { days before survey } \\
\text { and took treatment } \\
(\mathrm{N}=834 / 1631,51 \%)\end{array}$ & $\begin{array}{l}\text { Reported fever during } \\
\text { previous month } \\
(\mathrm{N}=117 / 2830,4 \%)\end{array}$ & $\begin{array}{l}\text { Reported fever starting } \\
>2 \text { days before survey } \\
\text { and took treatment } \\
(\mathrm{N}=99 / 117,85 \%)\end{array}$ \\
\hline \multicolumn{5}{|l|}{ Age (years) } \\
\hline $0-4$ & $405(25 \%)$ & $205(25 \%)$ & $23(20 \%)$ & $15(15 \%)$ \\
\hline $5-14$ & $306(19 \%)$ & $148(18 \%)$ & $29(25 \%)$ & $26(26 \%)$ \\
\hline $15+$ & $920(56 \%)$ & $481(58 \%)$ & $65(56 \%)$ & $58(59 \%)$ \\
\hline Sex (Female) & $850(52 \%)$ & $433(52 \%)$ & $63(54 \%)$ & $55(56 \%)$ \\
\hline Pregnant (Yes) & $39(2 \%)$ & $23(3 \%)$ & $3 / 116(3 \%)$ & $3 / 98(3 \%)$ \\
\hline \multicolumn{5}{|l|}{ Place of birth } \\
\hline Highland Papuan & $385(24 \%)$ & $172(21 \%)$ & $14(12 \%)$ & $11(11 \%)$ \\
\hline Lowland Papuan & $463(28 \%)$ & $183(22 \%)$ & $28(24 \%)$ & $19(19 \%)$ \\
\hline Non-Papuan & $783(48 \%)$ & $479(57 \%)$ & $75(64 \%)$ & $69(70 \%)$ \\
\hline Resided in lowlands for more than one year? (Yes) & $1547(95 \%)$ & $797(96 \%)$ & $111(95 \%)$ & $93(94 \%)$ \\
\hline \multicolumn{5}{|l|}{ Sub-district } \\
\hline Banti & $29(2 \%)$ & $18(2 \%)$ & $0(0 \%)$ & $0(0 \%)$ \\
\hline Harapan \& Kwamki Lama & $226(14 \%)$ & $124(15 \%)$ & $4(3 \%)$ & $2(2 \%)$ \\
\hline Inauga & $183(11 \%)$ & $106(13 \%)$ & $5(4 \%)$ & $5(5 \%)$ \\
\hline Kamoro Jaya & $107(7 \%)$ & $77(9 \%)$ & $12(10 \%)$ & $10(10 \%)$ \\
\hline Kadun Jaya, Kaugapu \& Pigapu & $108(7 \%)$ & $11(1 \%)$ & $23(20 \%)$ & $14(14 \%)$ \\
\hline Limau Asri, Iwaka, Mulia Kencana \& Naena Muktipura & $55(3 \%)$ & $3(0.4 \%)$ & $15(13 \%)$ & $15(15 \%)$ \\
\hline Koperapoka & $396(24 \%)$ & $127(15 \%)$ & $13(11 \%)$ & $12(12 \%)$ \\
\hline Timika Jaya, Kwamki \& Kwamki Baru & $383(23 \%)$ & $262(31 \%)$ & $16(14 \%)$ & $14(14 \%)$ \\
\hline Wonosari Jaya \& Nawaripi & $49(3 \%)$ & $39(5 \%)$ & $24(21 \%)$ & $23(23 \%)$ \\
\hline Karang Senang \& Bhintuka & $95(6 \%)$ & $67(8 \%)$ & $5(4 \%)$ & $4(4 \%)$ \\
\hline
\end{tabular}


Table 3.4: Multivariable analysis of risk factors for febrile illness in the past month $(\mathrm{N}=2890)$.

\begin{tabular}{lllll}
\hline Variable & OR $(95 \%$ CI $)$ & p value & AOR $(95 \%$ CI $)$ & p value \\
\hline Gender & &
\end{tabular}

Female

Male

Age

$$
\begin{aligned}
& \geq 15 \text { years } \\
& 5-14 \text { years } \\
& <5 \text { years }
\end{aligned}
$$

\section{Pregnant}

No

Yes

$\begin{array}{rr}\text { Reference } & \\ 1.24(0.85-1.82) & 0.266 \\ \text { Reference } & \\ 1.08(0.68-1.70) & 0.746 \\ 1.02(0.61-1.69) & 0.951\end{array}$

Reference

$1.69(0.51-5.55)$

0.391

Household size

$>7$ members

$\leq 7$ members

SES groups

Richest

Fourth

Middle

Second

Poorest

\section{Ethnicity}

Highland Papuan

Lowland Papuan

Non-Papuan
Reference

$2.11(1.01-4.43)$
0.951

$-$

\section{Resided in lowlands $>1$ year?}

Yes

Reference

No

$1.24(0.54-2.84)$

0.605

$\mathbf{0 . 0 4 8} 2.00(0.96-4.15) \quad 0.062$

Reference

Reference $0.68(0.37-1.24) \quad 0.211$

$1.32(0.78-2.23)$

0.302

0.857

0.410

Family sleeps under a bednet?

$\mathrm{No}$

Reference

Yes

$1.27(0.86-1.86)$

Reference

$\begin{array}{lll}0.275 & 1.53(0.79-2.97) & 0.209\end{array}$

$\mathbf{0 . 0 2 5} 1.84(1.05-3.26)$

0.035

The multiple logistic regression included variables that were significant $(\mathrm{p}<0.05)$ in the univariate logistic regression. 
Table 3.5: Changes in parasite prevalence by location (subdistrict).

\begin{tabular}{|c|c|c|c|c|c|c|c|c|c|c|c|c|}
\hline \multirow{3}{*}{ Subdistrict } & \multicolumn{6}{|c|}{2005} & \multicolumn{6}{|c|}{2013} \\
\hline & \multirow{2}{*}{$\begin{array}{l}\text { Household } \\
\text { members }\end{array}$} & \multirow{2}{*}{$\begin{array}{l}\text { Total } \\
\text { parasitaemic } \\
\mathrm{N}(\%) \\
\end{array}$} & \multicolumn{4}{|c|}{ Species - n (\%) } & \multirow[b]{2}{*}{$\begin{array}{l}\text { Household } \\
\text { members }\end{array}$} & \multirow{2}{*}{$\begin{array}{l}\text { Total } \\
\text { parasitaemic } \\
\mathrm{N}(\%)\end{array}$} & \multicolumn{4}{|c|}{ Species - n (\%) } \\
\hline & & & P. falciparum & P. vivax & P. malariae & $\begin{array}{l}\text { Mixed } \\
\text { infection }\end{array}$ & & & P. falciparum & P. vivax & P. malariae & $\begin{array}{l}\text { Mixed } \\
\text { infection }\end{array}$ \\
\hline Banti & 119 & $7(6 \%)$ & $0(0 \%)$ & $7(100 \%)$ & $0(0 \%)$ & $0(0 \%)$ & 0 & - & - & - & - & - \\
\hline Harapan \& Kwamki Lama & 576 & $136(24 \%)$ & $67(49 \%)$ & $56(41 \%)$ & $2(2 \%)$ & $11(8 \%)$ & 80 & $14(18 \%)$ & $6(43 \%)$ & $4(29 \%)$ & $4(29 \%)$ & $0(0 \%)$ \\
\hline Inauga & 405 & $47(12 \%)$ & $19(40 \%)$ & $23(49 \%)$ & $0(0 \%)$ & $5(11 \%)$ & 290 & $25(9 \%)$ & $10(40 \%)$ & $14(56 \%)$ & $1(4 \%)$ & $0(0 \%)$ \\
\hline Kamoro Jaya & 273 & $102(37 \%)$ & $44(43 \%)$ & $24(24 \%)$ & $11(11 \%)$ & $23(23 \%)$ & 254 & $37(15 \%)$ & $17(46 \%)$ & $14(38 \%)$ & $5(14 \%)$ & $1(3 \%)$ \\
\hline $\begin{array}{l}\text { Kadun Jaya, Kaugapu } \\
\& \text { Pigapu }\end{array}$ & 285 & $35(12 \%)$ & $10(29 \%)$ & $17(49 \%)$ & $2(6 \%)$ & $6(17 \%)$ & 198 & $60(30 \%)$ & $29(48 \%)$ & $25(42 \%)$ & $1(2 \%)$ & $5(8 \%)$ \\
\hline $\begin{array}{l}\text { Limau Asri, Iwaka, Mulia } \\
\text { Kencana \& Naena Muktipura }\end{array}$ & 111 & $5(5 \%)$ & $3(60 \%)$ & $2(40 \%)$ & $0(0 \%)$ & $0(0 \%)$ & 330 & $27(8 \%)$ & $17(63 \%)$ & $9(33 \%)$ & $1(4 \%)$ & $0(0 \%)$ \\
\hline Koperapoka & 726 & $100(14 \%)$ & $42(42 \%)$ & $45(45 \%)$ & $1(1 \%)$ & $12(12 \%)$ & 517 & $58(11 \%)$ & $27(47 \%)$ & $31(53 \%)$ & $0(0 \%)$ & $0(0 \%)$ \\
\hline $\begin{array}{l}\text { Timika Jaya, Kwamki \& } \\
\text { Kwamki Baru }\end{array}$ & 1045 & $133(13 \%)$ & $68(51 \%)$ & $57(43 \%)$ & $1(0.8 \%)$ & $7(5 \%)$ & 695 & $60(9 \%)$ & $17(28 \%)$ & $40(67 \%)$ & $2(3 \%)$ & $1(2 \%)$ \\
\hline Wonosari Jaya \& Nawaripi & 98 & $15(15 \%)$ & $11(73 \%)$ & $4(27 \%)$ & $0(0 \%)$ & $0(0 \%)$ & 258 & $37(14 \%)$ & $14(38 \%)$ & $16(43 \%)$ & $6(16 \%)$ & $1(3 \%)$ \\
\hline Karang Senang \& Bhintuka & 252 & $54(21 \%)$ & $26(48 \%)$ & $13(24 \%)$ & $7(13 \%)$ & $8(15 \%)$ & 173 & $30(17 \%)$ & $12(40 \%)$ & $10(33 \%)$ & $5(17 \%)$ & $3(10 \%)$ \\
\hline Total & 3890 & $634(16 \%)$ & $290(46 \%)$ & $248(39 \%)$ & $24(4 \%)$ & $72(11 \%)$ & 2795 & $348(12 \%)$ & $149(43 \%)$ & $163(47 \%)$ & $25(7 \%)$ & $11(3 \%)$ \\
\hline
\end{tabular}


0.001; Table 3.6). Malaria testing was positive in $92 \%(87 / 95)$ of cases and in one visit the result was not reported. P. falciparum was diagnosed in 53\% (46/87) of cases, $P$. vivax in $43 \%(37 / 87)$, mixed infections in $3 \%(3 / 87)$, and P. malariae in $1 \%(1 / 87)$. The increase in likelihood of individuals being tested for malaria was most apparent in the private sector where $29 \%(148 / 519)$ of individuals were tested in 2005 compared to $97 \%(31 / 32)$ in $2013(\mathrm{p}<0.001)$.

\subsubsection{Antimalarial treatment at healthcare providers}

In 2013 an antimalarial (alone or with other medicines) was reportedly prescribed during $88 \%(88 / 100)$ of visits to healthcare providers. Of these prescriptions, 95\% $(84 / 88)$ were prescribed as tablets, $1 \%(1 / 88)$ as an injection and $3 \%(3 / 88)$ as both an injection and tablets. Of the four malaria injections that were reported, two were at the public hospital and two at private providers. Ninety-nine percent $(87 / 88)$ of antimalarial prescriptions followed a blood test as per clinical guidelines; all were reportedly positive. This was a significant increase compared to 2005 when only $61 \%$ $(336 / 547)$ of prescriptions followed a blood test $(\mathrm{p}<0.001)$. In 2013 the antimalarial treatment regimen was prescribed correctly in $74 \%(64 / 86)$ of healthcare encounters in which $P$. vivax, P. falciparum or mixed infections were diagnosed, a significant increase from 2005 when only $23 \%$ (78/336) of individuals were correctly treated according to the prevailing guidelines $(\mathrm{AOR}=15.71$, [95\% CI: 8.18-30.18], $\mathrm{p}<0.001$; Table 3.6). These improvements in prescribing habits were apparent in both the public (AOR = 15.93 [95\% CI: 6.84-37.14], $\mathrm{p}<0.001)$ and private sectors $(\mathrm{AOR}=12.16[95 \% \mathrm{CI}$ : $3.42-43.28], \mathrm{p}<0.001)$.

The reported treatments prescribed for the different species are presented in Table 3.7. In $201395 \%$ (57/60) of patients diagnosed with malaria in a public facility were treated with DHP compared to $52 \%(14 / 27)$ of those treated at a private provider $(\mathrm{p}<0.001)$. Between 2005 and 2013 the percentage of individuals reportedly being treated with 14 days of primaquine for $P$. vivax infection (alone or mixed) rose from $9 \%(16 / 169)$ to $63 \%(25 / 40) ;(\mathrm{AOR}=37.49$ [95\% CI: 9.94-141.41], $\mathrm{p}<0.001)$. Over 
Table 3.6: Percentage of respondents who reported receiving blood tests and antimalarials (including tablets and injections) by survey.

\begin{tabular}{|c|c|c|c|c|c|c|c|c|c|c|c|c|}
\hline & \multicolumn{4}{|c|}{ Public } & \multicolumn{4}{|c|}{ Private } & \multicolumn{4}{|c|}{ Overall } \\
\hline & 2005 & 2013 & $\operatorname{AOR}(95 \% \mathrm{CI})^{*}$ & $\mathrm{p}$ value & 2005 & 2013 & $\operatorname{AOR}(95 \% \mathrm{CI})^{*}$ & $\mathrm{p}$ value & 2005 & 2013 & $\operatorname{AOR}(95 \% \mathrm{CI})^{*}$ & $\mathrm{p}$ value \\
\hline $\begin{array}{l}\text { Percent of visits that } \\
\text { resulted in a blood test } \\
\text { for malaria }\end{array}$ & $\begin{array}{l}76 \% \\
(285 / 375)\end{array}$ & $\begin{array}{l}94 \% \\
(64 / 68)\end{array}$ & - & $<0.001$ & $\begin{array}{l}29 \% \\
(148 / 519)\end{array}$ & $\begin{array}{l}97 \% \\
(31 / 32)\end{array}$ & - & $<0.001$ & $\begin{array}{l}48 \% \\
(433 / 894)\end{array}$ & $\begin{array}{l}95 \% \\
(95 / 100)\end{array}$ & $\begin{array}{l}25.42 \\
(9.88-65.42)\end{array}$ & $<0.001$ \\
\hline $\begin{array}{l}\text { Percent of blood tests } \\
\text { that were positive }\end{array}$ & $\begin{array}{l}75 \% \\
(213 / 285)\end{array}$ & $\begin{array}{l}94 \% \\
(60 / 64)\end{array}$ & - & $<0.001$ & $\begin{array}{l}84 \% \\
(124 / 148)\end{array}$ & $\begin{array}{l}87 \% \\
(27 / 31)\end{array}$ & - & 0.790 & $\begin{array}{l}78 \% \\
(337 / 433)\end{array}$ & $\begin{array}{l}92 \% \\
(87 / 95)\end{array}$ & $\begin{array}{l}3.11 \\
(1.29-7.47)\end{array}$ & 0.012 \\
\hline $\begin{array}{l}\text { Percent receiving } \\
\text { correct antimalarial } \\
\text { after testing positive } \dagger\end{array}$ & $\begin{array}{l}32 \% \\
(67 / 212)\end{array}$ & $\begin{array}{l}85 \% \\
(50 / 59)\end{array}$ & $\begin{array}{l}15.94 \\
(6.84-37.14)\end{array}$ & $<0.001$ & $\begin{array}{l}9 \% \\
(11 / 124)\end{array}$ & $\begin{array}{l}52 \% \\
(14 / 27)\end{array}$ & $\begin{array}{l}12.16 \\
(3.42-43.28)\end{array}$ & $<0.001$ & $\begin{array}{l}23 \% \\
(78 / 336)\end{array}$ & $\begin{array}{l}74 \% \\
(64 / 86)\end{array}$ & $\begin{array}{l}15.71 \\
(8.18-30.18)\end{array}$ & $<0.001$ \\
\hline $\begin{array}{l}\text { Percent receiving } \\
\text { chloroquine after } \\
\text { testing positive }\end{array}$ & $\begin{array}{l}62 \% \\
(133 / 212)\end{array}$ & $\begin{array}{l}0 \% \\
(0 / 59)\end{array}$ & - & $<0.001$ & $\begin{array}{l}43 \% \\
(53 / 124)\end{array}$ & $\begin{array}{l}11 \% \\
(3 / 27)\end{array}$ & - & 0.002 & $\begin{array}{l}55 \% \\
(186 / 336)\end{array}$ & $\begin{array}{l}3 \% \\
(3 / 86)\end{array}$ & - & $<0.001$ \\
\hline $\begin{array}{l}\text { Percent not receiving } \\
\text { an antimalarial after } \\
\text { testing negative }\end{array}$ & $\begin{array}{l}98 \% \\
(61 / 62)\end{array}$ & $\begin{array}{l}100 \% \\
(3 / 3)\end{array}$ & - & $-\ddagger$ & $\begin{array}{l}86 \% \\
(19 / 22)\end{array}$ & $\begin{array}{l}100 \% \\
(4 / 4)\end{array}$ & - & $-\ddagger$ & $\begin{array}{l}95 \% \\
(80 / 84)\end{array}$ & $\begin{array}{l}100 \% \\
(7 / 7)\end{array}$ & - & $-\ddagger$ \\
\hline $\begin{array}{l}\text { Percent not receiving } \\
\text { an antimalarial if no } \\
\text { blood test given }\end{array}$ & $\begin{array}{l}64 \% \\
(58 / 90)\end{array}$ & $\begin{array}{l}100 \% \\
(4 / 4)\end{array}$ & - & $-\ddagger$ & $\begin{array}{l}52 \% \\
(192 / 371)\end{array}$ & $\begin{array}{l}0 \% \\
(0 / 1)\end{array}$ & - & $-\ddagger$ & $\begin{array}{l}54 \% \\
(250 / 461)\end{array}$ & $\begin{array}{l}80 \% \\
(4 / 5)\end{array}$ & - & 0.383 \\
\hline
\end{tabular}

* Multivariate logistic regression adjusted for age, sex, ethnicity and household SES status if five or more observations. Fischer's Exact if less than five observations (no AORs).

$\dagger$ In 2005 local guidelines recommended antimalarial treatment with chloroquine plus sulphadoxine-pyramethamine for P. falciparum and chloroquine for $P$. vivax. In those patients with $P$. vivax (either alone or mixed) primaquine was also recommended. In 2013 correct treatment regimens were dihydoartemisinin-piperaquine for all species of malaria plus primaquine in those with $P$. vivax infections (alone or mixed). P. malariae infections $(\mathrm{n}=2)$ were excluded from this analysis.

$\$ 2013$ data predicted the outcome perfectly. All individuals had the same level of outcome; hence, no statistical computations can be performed for the logistic regression - no statistical output is given in software. 
Table 3.7: Reported antimalarial treatment for individuals by reported diagnosis.

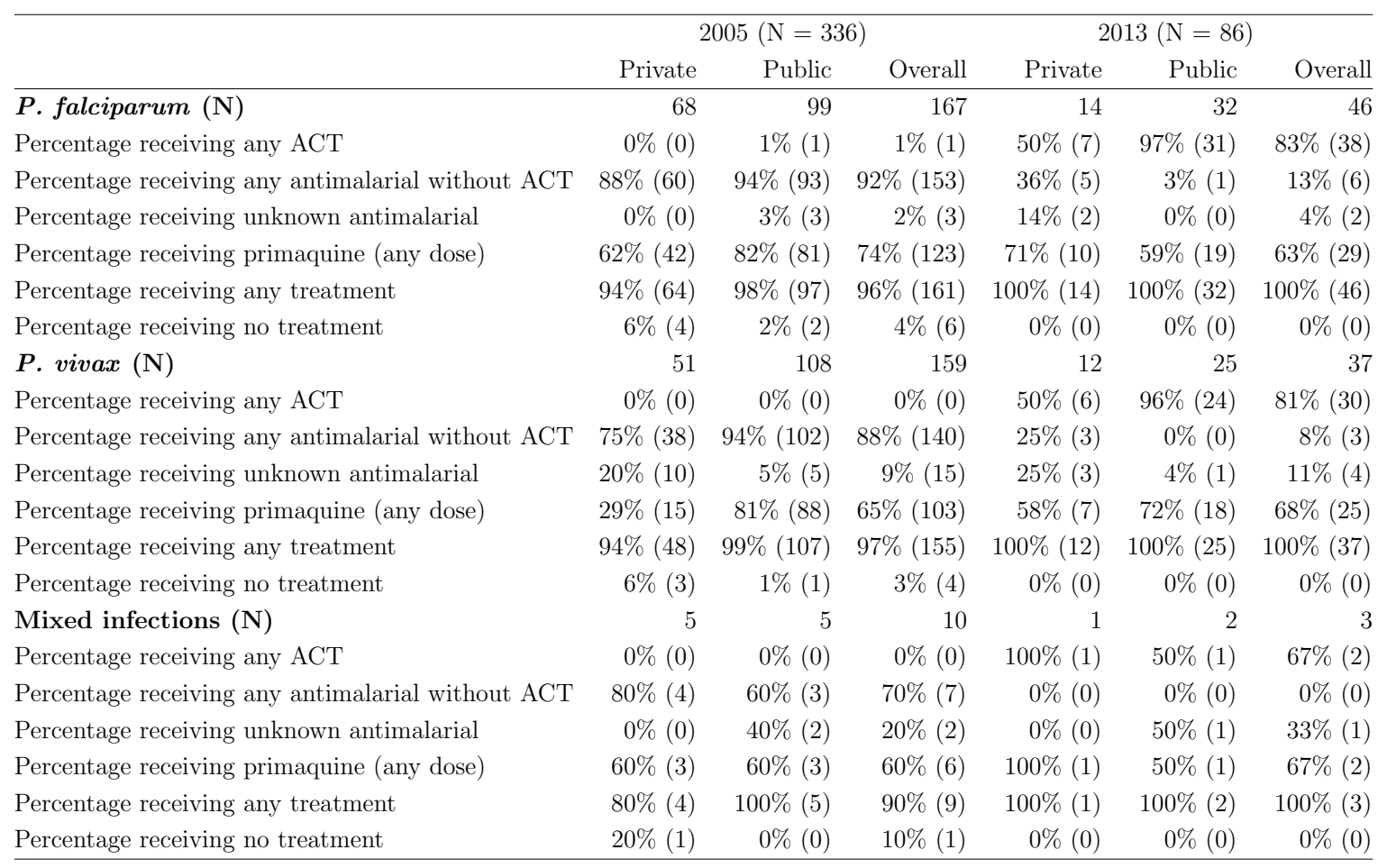

the same period, the percentage of patients with $P$. falciparum reporting treatment with a single dose primaquine rose from $19 \%(31 / 167)$ in 2005 to $50 \%(23 / 46)$ in 2013 $(\mathrm{AOR}=6.27$ [95\% CI: 2.68-14.68], $\mathrm{p}<0.001)$.

\subsubsection{Cost burden of fever}

In 2013 a total of 100 visits to healthcare providers were reported by 98 individuals; hence, nearly all patients reported requiring only one visit compared to $14 \%(107 / 764)$ of individuals who reported seeking medical care at two or more providers before the policy change $(\mathrm{p}<0.001)$. In 2013 the mean total direct cost to the household per visit was $\mathrm{US} \$ 16.8(\mathrm{SD}=19.0)$ at a private provider and $\mathrm{US} \$ 4.1(\mathrm{SD}=14.4)$ at the public sector $(\mathrm{p}<0.001)$. The mean daily wage in 2013 was $\mathrm{US} \$ 18.2(\mathrm{SD}=12.5)$, with a total mean indirect cost per fever episode of US\$52.5 ( $\mathrm{SD}=41.4)$ for adults and $\mathrm{US} \$ 7.3(\mathrm{SD}=16.4)$ for children.

Table 3.8 shows the cost per fever episode resulting in treatment seeking in 2005 and 2013. While the direct costs were similar $(\mathrm{p}=0.119)$, a significant decrease in indirect costs was evident from US\$44.2 (SD $=51.0)$ before the policy change to US\$33.8 (SD 
Table 3.8: Reported direct, indirect and total costs per individual taking treatment for fever over the entire fever episode (2014 US\$).

\begin{tabular}{lllllr}
\hline & \multicolumn{2}{c}{$2005(\mathrm{~N}=834)$} & \multicolumn{2}{c}{$2013(\mathrm{~N}=99)$} & \\
Category & Mean $(\mathrm{SD})$ & Median $(\mathrm{IQR})$ & Mean $(\mathrm{SD})$ & Median $(\mathrm{IQR})$ & $\mathrm{p}$ value* \\
\hline Total direct costs $\dagger$ & $9.4(27.3)$ & $1.9(0.4-5.6)$ & $8.1(17.0)$ & $1.7(1.0-8.1)$ & 0.119 \\
$\begin{array}{l}\text { Total indirect costs } \\
\text { Total cost to the individual taking }\end{array}$ & $44.2(51.0)$ & $32.8(10.9-60.1)$ & $33.8(40.1)$ & $18.2(0.0-54.6)$ & $\mathbf{0 . 0 0 6}$ \\
$\quad$ treatment due to lost wages & $19.3(30.6)$ & $0.0(0.0-32.8)$ & $28.4(38.3)$ & $9.1(0.0-45.5)$ & $\mathbf{0 . 0 1 0}$ \\
Total cost for companions & $4.1(6.5)$ & $0.00(0.0-5.5)$ & $3.2(7.6)$ & $0.0(0.0-0.0)$ & $\mathbf{0 . 0 0 1}$ \\
Total cost for caretaking & $19.7(30.5)$ & $10.9(0.0-27.3)$ & $2.0(9.1)$ & $0.0(0.0-0.0)$ & $<\mathbf{0 . 0 0 1}$ \\
Total cost for substitute labor & $1.1(6.5)$ & $0.0(0.0-0.0)$ & $0.2(1.8)$ & $0.0(0.0-0.0)$ & 0.072 \\
Total costs & $53.5(65.3)$ & $33.8(16.4-66.7)$ & $41.9(44.3)$ & $27.3(8.1-64.7)$ & 0.104 \\
\hline
\end{tabular}

* Mann-Whitney rank test.

$\dagger$ Includes payments made by the household for all treatments including diagnostics and transport.

$=40.1)$ in $2013(\mathrm{p}=0.006)$. In 2013 only $29 \%(29 / 99)$ of individuals reported that someone had to cut back on their usual activities to care for them, a decrease from $77 \%(641 / 834)$ in $2005(\mathrm{OR}=0.12$ [95\% CI: 0.08-0.20], $\mathrm{p}<0.001)$. There was also a reduction in the number of individuals requiring a substitute laborer from $8 \%(66 / 834)$ in 2005 to $1.0 \%(1 / 99)$ in $2013(\mathrm{p}=0.007)$. There was no significant difference in the proportion of individuals having at least one companion accompany them to seek treatment $(66 \%$ [553/834] in 2005 versus $73 \%$ [72/99] in 2013; $\mathrm{p}=0.200)$. Overall, the mean cost per fever episode was US\$41.9 $(\mathrm{SD}=44.3)$ in 2013 compared to US\$53.5 $(\mathrm{SD}=65.3)$ in $2005(\mathrm{p}=0.104)$.

\subsection{Discussion}

The results of the household surveys undertaken before and after implementation of ACT after confirmed diagnosis in Papua, Indonesia highlight several important changes in treatment-seeking behaviour, diagnosis of malaria and adherence to antimalarial drugs. Only one individual (1\%) did not seek treatment outside of the home, compared to $8 \%$ in 2005 when less efficacious treatments were available in both the public and private sectors. This shift in treatment seeking was associated with more individuals attending public providers at least once, rising from $46 \%$ in 2005 to $67 \%$ in 2013 . Those 
from households with lower SES were still more likely to seek treatment at public providers than those with higher SES.

Before the implementation of ACT, more than $40 \%$ of patients failed antimalarial treatment within 42 days, with almost half of these patients doing so within seven days [5]. The introduction of a three-day regimen of DHP resulted in rapid parasite clearance and symptom recovery with less than $5 \%$ of patients having recurrent malaria, most of which occurred after 28 days [9]. Hence, the observed changes in treatment-seeking behaviour likely reflect community awareness of such highly effective treatments and their availability initially in the public sector and subsequently in the private sector.

In parallel with the shift in where patients sought treatment there were also significant changes in the way in which healthcare services were provided, particularly the emphasis on only treating malaria after lab confirmation and adherence to antimalarial policy. RDTs were introduced at public health facilities without microscopists in 2006-07 and were used for half of the outpatient visits to one of the hospitals, whereas microscopy remained the main diagnostic for patients requiring admission to hospital. In 2013 individuals who attended healthcare facilities were nearly twice as likely to have a blood test for malaria compared to those in 2005, and this was particularly prominent in the private sector where almost all patients were offered a blood test in 2013. Considering that dissatisfaction with the public sector is common [19] and studies in Southeast Asia have shown that a large proportion of individuals seek treatment in the private sector $[20,21]$, improvement of both sectors is vital to ensuring public health benefits [22].

Our findings also demonstrate that those diagnosed with malaria in 2013 were threefold more likely to receive the recommended treatment regimen. Importantly, both the public and private sectors implemented the policy change to ACT, with reductions in prescriptions of non-ACT antimalarials apparent in both sectors. This included an eight-fold decline in chloroquine prescriptions. Furthermore, better prescribing practices were also observed for primaquine, which is encouraged as a single dose for reducing the transmission potential of $P$. falciparum and as a 14 day regimen for the radical cure of P. vivax. 
The survey results also highlight a modest fall in the prevalence of parasitaemia from $16 \%$ to $12 \%$, although individuals who were parasitemic were six-fold less likely to be symptomatic. Furthermore, a 10-fold reduction (from $42 \%$ to $4 \%$ ) was observed in those reporting fever in the preceding month. Before policy change, individuals reporting recent fever were three times more likely to be parasitaemic at the time of the survey than those with no recent febrile illness. Since the risk of an infected mosquito bite within 28 days in this region is low $(<8 \%)$, the most likely explanation for this is that prior to 2006 treatment with partially effective treatment often failed to clear parasitaemia by the time of the survey, whereas this was no longer apparent after the change in policy to highly effective treatment with ACT. In contrast no reduction was observed in the prevalence of asymptomatic parasitaemia between 2005 and 2013, a likely reflection of these patients not being unwell enough to seek treatment [23].

The first survey was conducted between July and December, whereas the second was undertaken from April through July. While seasonal variation in either malaria or other causes of fever could therefore have influenced our observations, malaria transmission in this area occurs throughout the year with minimal seasonality [23]. Other factors that could have affected our results include fluctuations in the demography and an increase in the supply of care by public providers through the second hospital. Between 2005 and 2013 the population grew from 120,000 to 172,000, and this was complemented with a slight increase in the proportion of non-Papuans, who are generally less immune. But this was countered by individuals reporting having lived in the area longer which would facilitate greater asymptomatic infections. In 2013 the only significant risk factor for recent febrile illness was being non-Papuan (Table 3.4). Whereas in 2005 Lowland Papuans, children less than five years old, and those from lower SES household were all at greater risk (Chapter 2). The changes in treatment-seeking behaviour therefore likely reflect a complex shift in population dynamics coupled with the impact of better treatment regimens and healthcare provision.

The observed changes in treatment seeking had important implications for the household costs incurred by malaria. While the mean cost for each febrile episode 
decreased from US\$53.5 to US\$41.9, reported monthly income in households increased from $60 \%$ with less than US\$500 in 2005 to $49 \%$ with less than US\$560 in 2013. Furthermore, in 2005 households more frequently reported multiple household members with fevers in the past month and those household members reported more repeat visits as compared to 2013. Considering the decrease in symptomatic individuals who were parasite positive, it is likely that many of these changes in treatment-seeking behaviour are a result of effective treatment.

This study has a number of limitations. Firstly, a degree of recall bias is likely due to the time between the fever episode and the survey, though this bias would be expected to be similar for both surveys. Due to differences in the phrasing of the questionnaires, we were unable to explore how use of bed nets had changed over time. As with the previous survey, many older male household members were not present at the time of the interviews and temporary migrants were excluded.

Another key limitation is the eight year gap between surveys. While the crosssectional design quantified the changes in parasitaemia, treatment-seeking behaviour and costs from 2005 to 2013, the time interval between surveys increases confounding from other changes besides the policy change including demographic dynamics, such as smaller households, greater representation of females, and duration residing in the study area. As reflected in the above discussion, this gap makes it more challenging to infer a direct policy impact; however, public health and malaria control activities aimed at changing behaviour remained similar over this time period. Had it been possible to include a control group, an impact evaluation may have been a better result.

In conclusion, the policy change in March 2006 to a unified treatment of malaria with DHP had a significant impact on treatment-seeking behavior, resulting in more patients attending healthcare facilities where better case management was provided. These changes had a significant impact on the burden of malaria with fewer patients reporting febrile illness; however, asymptomatic infections increased. Further reductions in malaria and its ultimate elimination will require implementing more effective radical cure of $P$. vivax alongside active case detection or presumptive interventions. 


\subsection{Declarations}

\subsubsection{Acknowledgments}

I am grateful to Lembaga Pengembangan Masyarakat Amungme Kamoro, Morrison Bethea, and the staff of PT Freeport Indonesia Public Health \& Malaria Control Department and International SOS for support and technical assistance. I thank Mauritz Okeseray, Ferryanto Chalfein, Budi Prasetyorini, Peter Ebsworth and Michael Bangs for their support and technical assistance. I thank Dr. Mavuto Mukaka for statistical advice.

\subsubsection{Ethics approval}

This study was approved by the Ethics Committee of the National Institute of Health research and Development (NIHRD), Indonesian Ministry of Health and the Human Research (KS.02.01.2.1.2684), University of Gadjah Mada (KE/FK/763/EC), and the Ethics Committee of NT Department of Health \& Community Services and Menzies School of Health Research, Darwin, Australia (HREC 04/47 and 10-1434). Written informed consent was obtained from all adult participants and parents of enrolled children. 


\section{References for Chapter 3}

[1] Statistics Indonesia (Badan Pusat Statistik BPS) and National Population and Family Planning Board (BKKBN) and Kementerian Kesehatan (KemenkesMOH) and ICF International. Indonesia Demographic and Health Survey 2012.; 2013.

[2] World Health Organisation. Malaria in Indonesia; 2015. Available from: http://www.who.int/countries/idn/en/.

[3] Burdam FH, Hakimi M, Thio F, Kenangalem E, Indrawanti R, Noviyanti R, et al. Asymptomatic Vivax and Falciparum Parasitaemia with Helminth Co-Infection: Major Risk Factors for Anaemia in Early Life. PLoS One. 2016;11(8):e0160917. doi:10.1371/journal.pone.0160917.

[4] Pava Z, Burdam FH, Handayuni I, Trianty L, Utami RA, Tirta YK, et al. Submicroscopic and Asymptomatic Plasmodium Parasitaemia Associated with Significant Risk of Anaemia in Papua, Indonesia. PLoS One. 2016;11(10):e0165340. doi:10.1371/journal.pone.0165340.

[5] Ratcliff A, Siswantoro H, Kenangalem E, Wuwung M, Brockman A, Edstein MD, et al. Therapeutic response of multidrug-resistant Plasmodium falciparum and P. vivax to chloroquine and sulfadoxine-pyrimethamine in southern Papua, Indonesia. Trans R Soc Trop Med Hyg. 2007;101(4):351-9.

[6] Tjitra E, Baker J, Suprianto S, Cheng Q, Anstey NM. Therapeutic efficacies of artesunate-sulfadoxine-pyrimethamine and chloroquine-sulfadoxine-pyrimethamine in vivax malaria pilot studies: relationship to Plasmodium vivax dhfr mutations. Antimicrob Agents Chemother. 2002;46(12):3947-53.

[7] Sumawinata IW, Bernadeta, Leksana B, Sutamihardja A, Purnomo, Subianto B, et al. Very high risk of therapeutic failure with chloroquine for uncomplicated Plasmodium falciparum and P. vivax malaria in Indonesian Papua. Am J Trop Med Hyg. 2003;68(4):416-20. 
[8] Hasugian AR, Purba HL, Kenangalem E, Wuwung RM, Ebsworth EP, Maristela R, et al. Dihydroartemisinin-piperaquine versus artesunate-amodiaquine: superior efficacy and posttreatment prophylaxis against multidrug-resistant Plasmodium falciparum and Plasmodium vivax malaria. Clin Infect Dis. 2007;44(8):1067-74. doi:10.1086/512677.

[9] Ratcliff A, Siswantoro H, Kenangalem E, Maristela R, Wuwung RM, Laihad F, et al. Two fixed-dose artemisinin combinations for drug-resistant falciparum and vivax malaria in Papua, Indonesia: an open-label randomised comparison. Lancet. 2007;369(9563):757-65. doi:10.1016/s0140-6736(07)60160-3.

[10] Karyana M, Burdarm L, Yeung S, Kenangalem E, Wariker N, Maristela R, et al. Malaria morbidity in Papua Indonesia, an area with multidrug resistant Plasmodium vivax and Plasmodium falciparum. Malar J. 2008;7:148. doi:10.1186/1475-2875-7-148.

[11] Elyazar IR, Hay SI, Baird JK. Malaria distribution, prevalence, drug resistance and control in Indonesia. Adv Parasitol. 2011;74:41-175. doi:10.1016/b978-0-12-385897-9.00002-1.

[12] ORC Macro. Guidelines for Sampling for the Malaria Indicator Survey; 2005.

[13] StataCorp. Stata Statistical Software: Release 14. 2015;

[14] R Core Team. R: A Language and Environment for Statistical Computing. 2015; Available from: https ://www.R-project.org/.

[15] Jombart T, Devillard S, Balloux F. Discriminant analysis of principal components: a new method for the analysis of genetically structured populations. BMC Genet. 2010;11:94. doi:10.1186/1471-2156-11-94.

[16] OANDA. Currency Converter: Historical Exchance Rates for 2005;Available from: http://www.oanda.com/currency/historical-rates/(Accessed: 3 March 2015). 
[17] OECD. Exchange rates (indicator). 2017;Available from:

https://data.oecd.org/conversion/exchange-rates.htm. doi:OECD (2017), Exchange rates (indicator). doi: 10.1787/037ed317-en (Accessed on 17 March 2017).

[18] The World Bank. Inflation, consumer prices (annual \%). 2015;Available from: http://data.worldbank.org/indicator/FP.CPI.TOTL.ZG(Accessed: 4 August 2015).

[19] Williams HA, Jones CO. A critical review of behavioral issues related to malaria control in sub- Saharan Africa: what contributions have social scientists made? Soc Sci Med. 2004;59(3):501-23. doi:10.1016/j.socscimed.2003.11.010.

[20] ACTwatch Group and PSI/Cambodia. Kingdom of Cambodia Household Survey Report, 2011; 2011. Available from: www.actwatch.info.

[21] ACTwatch Group and PSI/Myanmar. The Republic of the Union of Myanmar Household Survey: Baseline, 2012. Population Services International; 2012. Available from: www.actwatch.info.

[22] Population Services International and ACTwatch. Malaria Market Trends in Sub-Saharan Africa: 2009-2014; 2015.

[23] Mimika DHO. Mimika District Health Office annual statistics.; 2005. 


\title{
Chapter 4
}

\section{Using G6PD tests to enable the}

\author{
safe treatment of $P$. vivax
}

\section{infections with primaquine on the}

\section{Thailand-Myanmar border: A}

\section{cost-effectiveness analysis}

This chapter been published as Devine A, Parmiter M, Chu CS, Bancone G, Nosten F, Price RN, Lubell Y, Yeung S. Using G6PD tests to enable the safe treatment of Plasmodium vivax infections with primaquine on the Thailand-Myanmar border: A cost-effectiveness analysis. PLoS Negl Trop Dis. 2017;11(5):e0005602. https://doi.org/10.1371/journal.pntd.0005602 PMID: 28542194.

\subsection{Introduction}

P. vivax is an important public health concern, particularly in Asia and South America, where it is now responsible for the majority of malaria cases. While traditionally regarded as a benign disease, $P$. vivax malaria has been associated with 
severe and fatal outcomes $[1,2]$. As countries move toward malaria elimination and the overall incidence of malaria declines, the proportion of cases that are due to $P$. vivax infections increases $[3,4]$. A single infection of $P$. vivax can lead to multiple relapses due to its ability to form dormant liver stage parasites called hypnozoites. These relapses are indistinguishable from new infections and repeated episodes can lead to a cumulative risk of anaemia and malnutrition $[5,6]$. In short latency relapse settings, the majority of $P$. vivax cases are thought to be due to relapses [7]. Primaquine is the only drug currently licensed for the radical cure of $P$. vivax; however, it can cause severe haemolysis in individuals with G6PD deficiency, a common genetic disorder [8] that is positively associated with P. vivax incidence [9]. The prevalence of G6PD deficiency varies from less than $1 \%$ to more than $30 \%$, with a mean of $8 \%$ in countries where malaria is endemic; equivalent to 350 million people worldwide [10]. G6PD deficiency is largely asymptomatic until individuals are exposed to oxidative stress from an external source, including certain drugs, such as primaquine, and foods, most notably fava beans [9]. The degree of enzyme deficiency varies widely depending upon the genotypic variant which varies with geographical region. A recent review found only 14 documented deaths attributable to primaquine use [11]; however, fatalities may have gone unreported [12].

The WHO recommends that primaquine be used for the radical cure of $P$. vivax infected patients who can be tested for G6PD deficiency [13]. The gold standard for diagnosing G6PD deficiency is the spectrophotometric assay, a test that requires a laboratory setting and specialised staff $[14,15]$. The fluorescent spot test (FST), which is the most widely used assay for G6PD deficiency, is easier to perform but requires basic laboratory equipment, electricity and a cold chain, rendering it difficult to use in remote settings. Thus, routine testing for G6PD deficiency prior to prescribing primaquine generally is not part of antimalarial policy in most countries [16]. Recently, the CareStart G6PD (Access Bio, Somerset, NJ, USA) lateral flow RDT has become available for point-of-care testing. This phenotypic test has high sensitivity for an enzyme activity cut off of $30 \%$ [17, 18]; hence false negative results would rarely lead to 
a G6PD deficient individual receiving primaquine with an attendant risk of haemolysis. Unlike the other G6PD RDT by BinaxNOW (Alere, Orlando, FL, USA), the CareStart RDT can be used in settings where the temperature is above $25^{\circ} \mathrm{C}$, a common necessity in $P$. vivax endemic settings $[19,20]$.

The availability of point-of-care G6PD tests is of clinical and public health importance so that $P$. vivax patients have safe access to primaquine treatment for the prevention of relapses and the resulting health complications [20]. Here I evaluate the cost-effectiveness of using G6PD RDTs on the Thailand-Myanmar border and present our model as an interactive web tool that can be adapted to other settings.

\subsection{Methods}

\subsubsection{Base case analysis}

A cost-effectiveness analysis [21] using a health care provider perspective was conducted with decision tree models for $P$. vivax infections using $\mathrm{R}$ statistical software [22] over a one year time horizon. The model was parameterised for the north-western border of Thailand with Myanmar (Tak Province), with the benefit of data on recurrences from a recent clinical trial at the Shoklo Malaria Research Unit (SMRU), which provides free of charge care to migrants and refugees [23, 24]. This study was chosen as it compared chloroquine with chlorquine plus high-dose primaquine and followed patients for one year following their initial episode. Few previous studies can provide information over such a long time period [25].

In this population of migrants and refugees, the prevalence of G6PD deficiency was documented to be 9-18\% [26]. The most common genetic variant was the Mahidol variant (88\%) with Chinese-4, Viangchan, Aores, Seattle, and Mediterranean variants also present [26]. Low, unstable P. vivax transmission is seen in this area [3] with a frequent relapse pattern [27]. In recent years, the overall number of malaria cases has been decreasing while the prevalence of $P$. vivax in the population has remained relatively stable at $9 \%[3]$. Routine practice along the border is to administer 14 days 
of supervised therapy with or without G6PD screening to patients able to attend the clinic; in practice this is a small proportion of the patients.

The testing of G6PD status with CareStart G6PD RDT before administering primaquine ("screening strategy") was compared to a strategy in which no G6PD test is performed and primaquine is not used at all ("chloroquine strategy"). In addition, the screening strategy is compared with a strategy where primaquine is given to all patients without testing for G6PD deficiency ("primaquine strategy") (Table 4.1). The chloroquine strategy and primaquine strategy were not directly compared to each other because (1) it is unlikely that in settings where the chloroquine strategy is used switching to the primaquine strategy would be a viable option due to the evident concerns about safety and (2) it is unlikely that settings where the primaquine strategy is used would consider changing to the chloroquine strategy due to its inability to achieve radical cure. While this is a deviation from standard practice for economic evaluations, it isolates the options that countries are considering, allowing a focus on the information relevant to their policy decisions.

Recurrences were recorded over a one year time period in patients who were treated with chloroquine alone, as compared with those who were treated with chloroquine plus 14 days of supervised primaquine for each $P$. vivax episode (0.5 milligrams $(\mathrm{mg}) /$ kilogram $(\mathrm{kg}) /$ day) $[23,24]$. The relative risk of having at least one recurrence following primaquine treatment was 0.22 as compared to those receiving chloroquine alone. For those who had at least one recurrence, the mean number of recurrences was 3.54 in the chloroquine arm and 1.16 in the primaquine arm. The model applies the inclusion criteria of the clinical trial, which was restricted to patients who were six months and older, not pregnant and presenting with uncomplicated $P$. vivax malaria [23]. The mean age in the clinical trial was 21 years; this was used for the disability-adjusted life-year (DALY) calculations for years of life lost [24]. The analysis and results were completed separately for males (Figure 4.1) and females (Figure 4.2) to account for their differences in risks and outcomes. Firstly, as pregnant females would not be prescribed primaquine due to the unknown G6PD status of the 
Table 4.1: Summary of key components of each strategy.

\begin{tabular}{|c|c|c|c|}
\hline & Chloroquine strategy & Primaquine strategy & Screening strategy \\
\hline \multicolumn{4}{|c|}{ Initial visit } \\
\hline Treatment & $\begin{array}{l}\text { Chloroquine given } \\
\text { to all. }\end{array}$ & $\begin{array}{l}\text { Chloroquine and supervised } \\
\text { primaquine therapy ( } 14 \text { day) } \\
\text { given to all. All individuals } \\
\text { with severe G6PD deficiency } \\
\text { stop primaquine therapy early. } \\
\text { Females with intermediate } \\
\text { G6PD deficiency who do not } \\
\text { have severe haemolysis } \\
\text { complete primaquine therapy. }\end{array}$ & $\begin{array}{l}\text { Chloroquine and supervised } \\
\text { primaquine therapy (14 day) } \\
\text { given to those who test G6PD } \\
\text { normal. Chloroquine and weekly } \\
\text { primaquine given to those who } \\
\text { test G6PD abnormal. Individuals } \\
\text { with severe G6PD deficiency who } \\
\text { are inadvertently given } 14 \text { day } \\
\text { primaquine will stop therapy } \\
\text { early. Females with intermediate } \\
\text { G6PD deficiency who do not have } \\
\text { severe haemolysis complete } \\
\text { primaquine therapy. }\end{array}$ \\
\hline \multicolumn{4}{|c|}{ Recurrences (all costs and DALYs are weighted by the proportion of severe cases) } \\
\hline Costs & Cost per recurrence. & $\begin{array}{l}\text { Cost per recurrence. } \\
\text { Supervised primaquine ( } 14 \\
\text { day) for those who are G6PD } \\
\text { normal. }\end{array}$ & $\begin{array}{l}\text { Cost per recurrence. Includes G6PD } \\
\text { RDT. Supervised primaquine ( } 14 \text { day) } \\
\text { for those who are G6PD true normal. } \\
\text { Supervised primaquine ( } 8 \text { weekly) } \\
\text { for those who test G6PD abnormal } \\
\text { and false normal. }\end{array}$ \\
\hline Effects & $\begin{array}{l}\text { All have the number } \\
\text { of recurrences for } \\
\text { chloroquine treatment. }\end{array}$ & $\begin{array}{l}\text { G6PD abnormal individuals } \\
\text { have the number of recurrences } \\
\text { for chloroquine treatment. } \\
\text { G6PD normal individuals have } \\
\text { the number of recurrences } \\
\text { for primaquine treatment. }\end{array}$ & $\begin{array}{l}\text { Those who test G6PD false normal } \\
\text { have the number of recurrences for } \\
\text { chloroquine treatment. All other } \\
\text { individuals have the number of } \\
\text { recurrences for primaquine } \\
\text { treatment. }\end{array}$ \\
\hline
\end{tabular}

fetus, the screening and primaquine strategies modelled the inclusion of a pregnancy test for all women of childbearing age who were unaware that they were pregnant. Those who were identified or known to be pregnant would be treated with chloroquine only and would not have a G6PD RDT. Secondly, since G6PD is an X-linked disorder, males who have deficiency are hemizygous while females can be either homozygous or heterozygous with a range of G6PD expression levels. Accordingly, G6PD deficiency was divided into two groups: severe (<30\% enzyme activity) and intermediate (30-69\% enzyme activity). Only females can have intermediate deficiency and the outcomes in this group were taken from heterozygotes. Studies have shown that the G6PD RDT with a cut off of $30 \%$ activity does not detect heterozygous females with intermediate activity $[16,18]$; accordingly, some women with intermediate deficiency who are identified as G6PD normal with currently available RDTs could be at risk of severe 


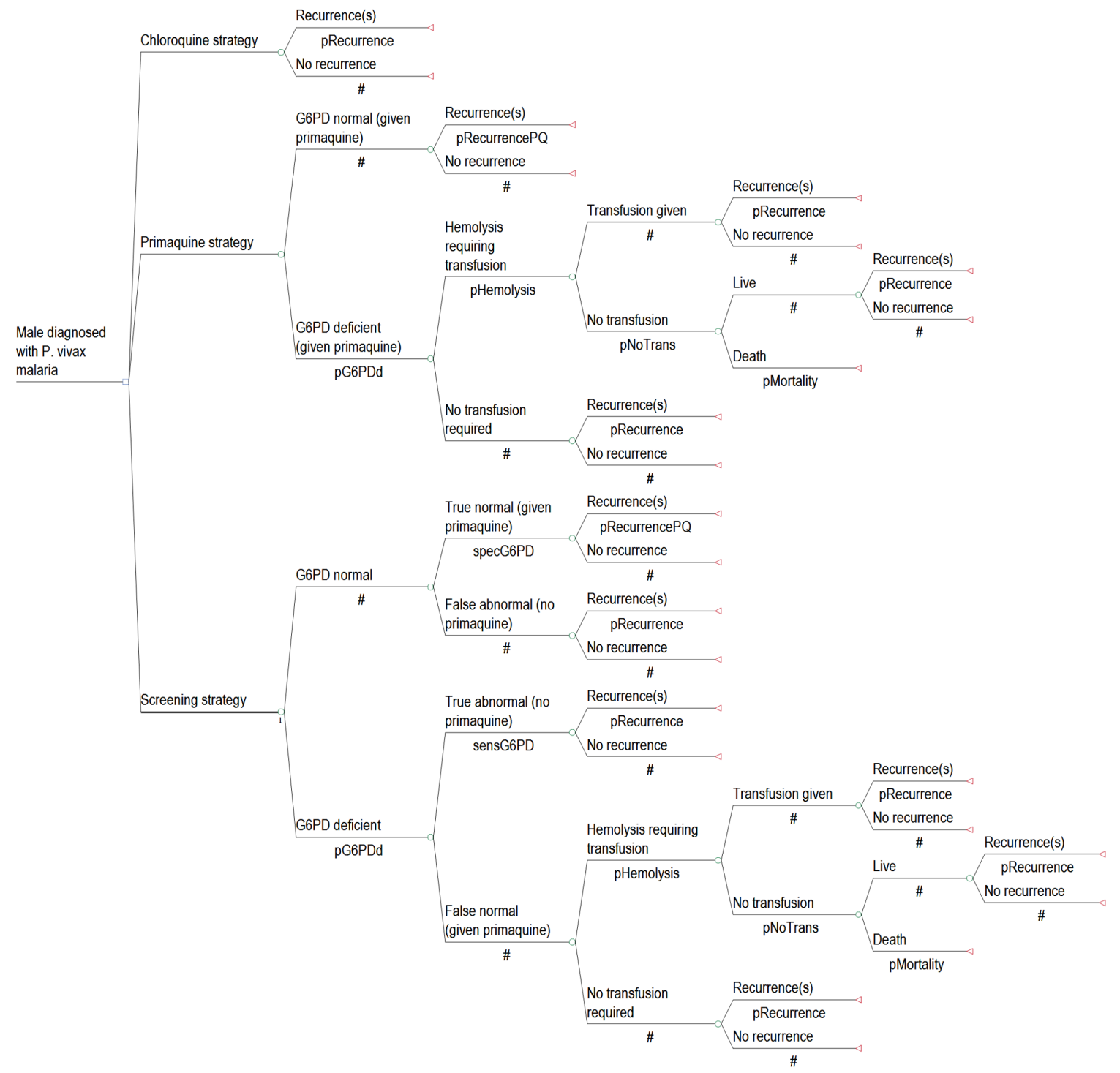

Figure 4.1: Model diagram of strategies for P. vivax treatment in males.

haemolysis when prescribed haemolytic drugs.

Table 4.2 shows the parameters used in the model. Both the screening and primaquine strategies include the cost of supervised therapy in order to reflect the additional costs required for the gains in effectiveness seen in the clinical trial. For the screening strategy, weekly supervised primaquine therapy for eight weeks was given to those who tested G6PD abnormal [28] and the effectiveness was taken from the trial results for 14 day therapy [24]. For each recurrence, the cost and DALY value used were taken from clinical episodes, severe malaria episodes and episodes that resulted in death and weighted proportionally. The probability for severe $P$. vivax was taken from a meta analysis of clinical studies using those with severe anaemia, but other symptoms due to 


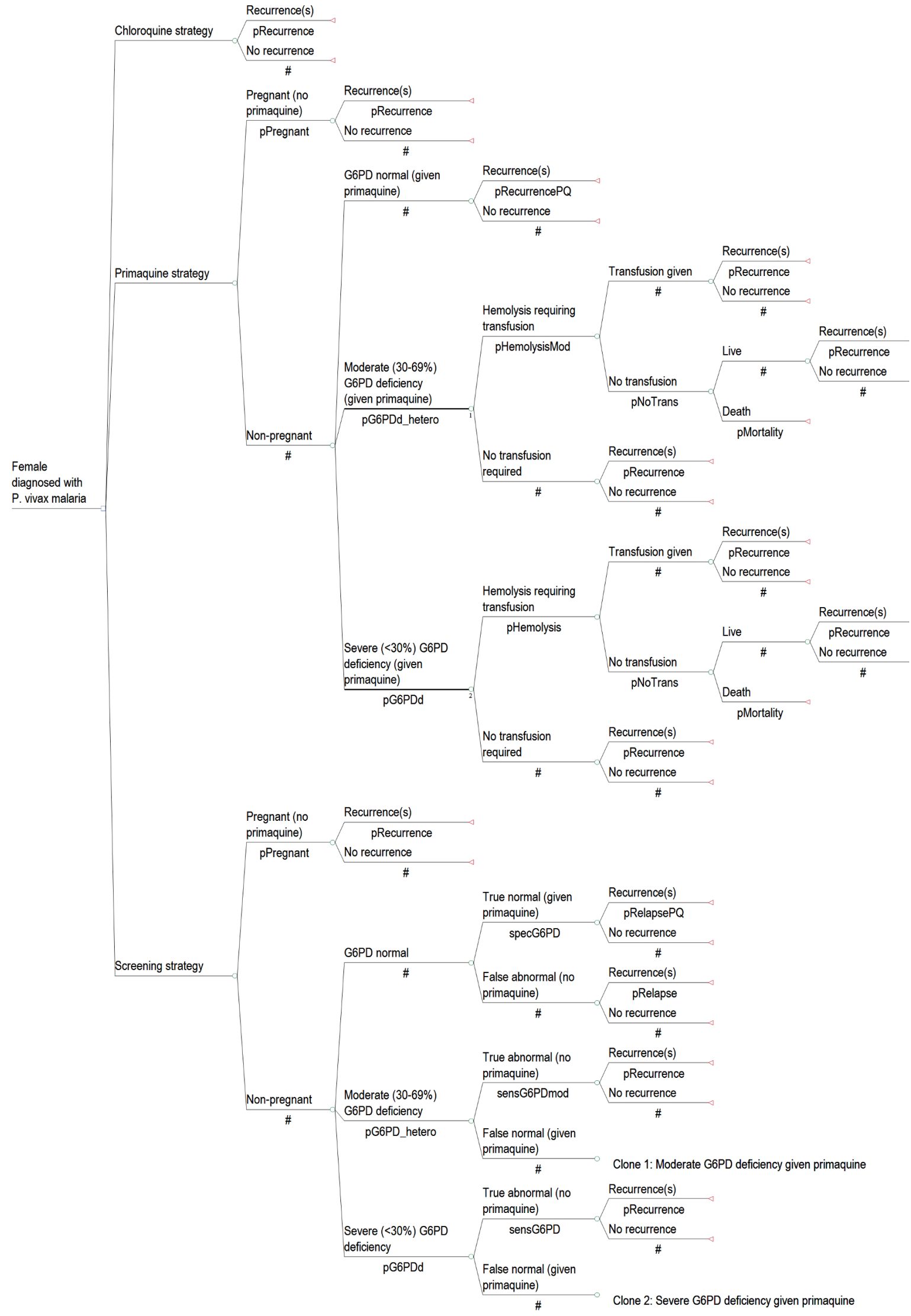

Figure 4.2: Model diagram of strategies for $P$. vivax treatment in females. 
severe $P$. vivax were not included [2].

The probability of having a haemolytic episode that requires a transfusion in individuals with severe and intermediate G6PD deficiency treated with primaquine was taken from a study of children treated with dapsone in Africa [29]. While this population may be different in terms of age and G6PD variant from those being treated with primaquine on the northwestern border of Thailand with Myanmar, this was the best available data on transfusion risk. The probability of haemolysis requiring transfusion for severe G6PD deficiency was taken from the proportion of hemizygotes and homozygotes in the study while the probability for females with intermediate deficiency was taken from the proportion of heterozygotes. It was assumed that $10 \%$ of patients requiring a transfusion did not receive one; of those, $10 \%$ died as a result of not receiving a transfusion. It was assumed that the decision to give a transfusion was made by a physician and that the costs are included in the cost of transfusion.

Costs of commodities and service delivery were taken from Myanmar and Thailand and supplemented by international sources when needed. Costs are reported in 2014 US $\$$. The cost of supervised therapy was taken from data on annual costs of a community health worker in Myanmar [34], assuming one half-day of pay per observation. The cost of hospitalisation for a blood transfusion was included for severe haemolytic episodes which did not lead to death. Table 4.1 describes the costs for recurrences. The DALY weights were taken from the 2010 Global Burden of Disease Study [40]. These weights were combined with life tables for Myanmar [38] and assumptions about the length of illness to calculate the DALY burden for each strategy.

In instances where the screening strategy averted DALYs while costing more money the incremental cost-effectiveness ratios (ICER) was calculated:

$$
I C E R=\frac{\text { Cost }_{s}-\text { Cost }_{b}}{D A L Y_{b}-D A L Y_{s}}
$$

Where Cost is the total cost of the strategy and DALY is the total DALYs of the corresponding strategy. While the gross domestic product per capita for Myanmar is approximately US $\$ 1,200$ [41], it has been argued that a lower WTP threshold may be 
Table 4.2: Model parameters and sources (2014 US\$).

\begin{tabular}{|c|c|c|c|}
\hline & Base case (low, high) & $\begin{array}{l}\text { Distribution } \\
\text { (Parameters*) }\end{array}$ & Sources \\
\hline Proportion of males with severe G6PD deficiency & $0.137 \pm 50 \%$ & Beta $(13.50,85.05)$ & {$[26]$} \\
\hline Proportion of females with severe G6PD deficiency & $0.050 \pm 50 \%$ & Beta $(15.17,288.18)$ & Females with $<30 \%$ activity (Section 4.6 ) \\
\hline Proportion of females with intermediate G6PD activity & $0.158 \pm 50 \%$ & Beta $(12.96,69.04)$ & Females with 30-69\% activity (Section 4.6) \\
\hline $\begin{array}{l}\text { Sensitivity of CareStart G6PD RDT for severe } \\
\text { deficiency }\end{array}$ & $0.99(0.9-1.00)$ & Beta $(9.39,0.09)$ & [18] (Low range from [16]) \\
\hline $\begin{array}{l}\text { Sensitivity of CareStart G6PD RDT for intermediate } \\
\text { deficiency }\end{array}$ & $0.44 \pm 50 \%$ & $\operatorname{Beta}(7.97,10.14)$ & {$[18]$} \\
\hline Specificity of CareStart G6PD RDT for males & $0.99(0.75-1.00)$ & Beta $(3.01,0.03)$ & [18] (Low range from [16]) \\
\hline Specificity of CareStart G6PD RDT for females & $0.97(0.68-0.99)$ & Beta $(2.59,0.08)$ & [18] (Low range from [16]) \\
\hline $\begin{array}{l}\text { Proportion who have at least one recurrence in the } \\
\text { following year if treated with CQ }\end{array}$ & 0.732 & - & {$[24]$} \\
\hline $\begin{array}{l}\text { Relative risk of having at least one recurrence in the } \\
\text { following year if treated with } \mathrm{CQ}+\mathrm{PQ}\end{array}$ & $0.219(0.158-1)$ & $\begin{array}{l}\text { Lognormal }(-1.52 \text {, } \\
0.47)\end{array}$ & {$[23,24]$} \\
\hline $\begin{array}{l}\text { Mean number of recurrences in those treated with } \\
\mathrm{CQ} \text { who have at least } 1 \text { recurrence }\end{array}$ & $3.537(1-8)$ & Bootstrapped data & $\begin{array}{l}{[23,24] \text { Each PSA iteration is the mean of }} \\
100 \text { bootstrapped data points. }\end{array}$ \\
\hline $\begin{array}{l}\text { Mean number of recurrences in those treated with } \\
\mathrm{CQ}+\mathrm{PQ} \text { who have at least } 1 \text { recurrence }\end{array}$ & $1.156(1-2)$ & Bootstrapped data & $\begin{array}{l}{[23,24] \text { Each PSA iteration is the mean of }} \\
100 \text { bootstrapped data points. }\end{array}$ \\
\hline $\begin{array}{l}\text { Proportion who need a blood transfusion after taking } \\
\text { primaquine if have severe G6PD deficiency }\end{array}$ & $0.109(0.007-0.15)$ & Beta $(10.40,85.01)$ & {$[29]$} \\
\hline $\begin{array}{l}\text { Proportion who need a blood transfusion after taking } \\
\text { primaquine if have intermediate G6PD deficiency }\end{array}$ & $0.005(0.001-0.05)$ & Beta $(0.09,18.72)$ & {$[29,30]$ Results for female heterozygotes } \\
\hline $\begin{array}{l}\text { Proportion who need a transfusion due to haemolysis } \\
\text { but do not receive it }\end{array}$ & $0.100(0.010-0.150)$ & Beta $(8.77,78.90)$ & $\begin{array}{l}\text { Assumption for both severe and intermediate } \\
\text { deficiency }\end{array}$ \\
\hline Mortality due to not receiving a needed transfusion & $0.100(0.010-0.500)$ & Beta $(2.22,19.95)$ & $\begin{array}{l}\text { Assumption for both severe and intermediate } \\
\text { deficiency }\end{array}$ \\
\hline Proportion of females who are pregnant & $0.11 \pm 50 \%$ & Beta $(14.05,113.65)$ & Assumption \\
\hline $\begin{array}{l}\text { Proportion of females who need to take a } \\
\text { pregnancy test }\end{array}$ & $0.50 \pm 50 \%$ & Beta $(6.92,6.92)$ & $\begin{array}{l}\text { Assumption. All women of childbearing age are } \\
\text { tested unless they disclose pregnancy }\end{array}$ \\
\hline \multicolumn{4}{|l|}{ COSTS } \\
\hline CareStart G6PD RDT & $1.75(0.88-10.00)$ & Gamma $(0.38,4.64)$ & RDT (1.50) [31] and administration (0.25) [32] \\
\hline Primaquine pill & $0.06 \pm 50 \%$ & Gamma $(24.71,0.00)$ & $\begin{array}{l}\text { [33] } 14 \text { days of } 30 \mathrm{mg} / \text { day; } 8 \text { weeks of } 45 \mathrm{mg} / \\
\text { week. Assumed weight }=60 \mathrm{~kg} \text {. }\end{array}$ \\
\hline $\begin{array}{l}\text { One session of supervised primaquine therapy by a } \\
\text { community health worker }\end{array}$ & $1.67 \pm 50 \%$ & Gamma $(16.15,0.10)$ & $\begin{array}{l}\text { [34] One-half day of work for a medium } \\
\text { accessible area with no incentives per } \\
\text { supervision }\end{array}$ \\
\hline Initial episode and clinical $P$. vivax recurrence & $7.86 \pm 50 \%$ & Gamma $(16.10,0.49)$ & $\begin{array}{l}\text { [35] Outpatient visit, excluding screening and } \\
\text { treatment costs }\end{array}$ \\
\hline Severe $P$. vivax recurrence & $\begin{array}{l}196.22(95 \% \text { CI: } \\
135.08-271.39)\end{array}$ & Gamma $(31.48,6.23)$ & [36] Inpatient visit for severe $P$. falciparum \\
\hline Haemolytic episode requiring transfusion & $320.71(166.03-401.53)$ & Gamma $(32.55,9.85)$ & $\begin{array}{l}\text { One unit of blood (25.31) [37] and } 7 \text { day } \\
\text { inpatient stay (295.40) [35] }\end{array}$ \\
\hline Pregnancy test & $0.10 \pm 50 \%$ & Gamma $(16.10,0.01)$ & SMRU clinic records \\
\hline \multicolumn{4}{|l|}{ DALYS } \\
\hline Proportion of $P$. vivax cases that are severe & $\begin{array}{l}0.020(95 \% \text { CI: } 0.013 \\
-0.027)\end{array}$ & $\begin{array}{l}\text { Beta }(31.41 \\
1539.05)\end{array}$ & {$[2]$} \\
\hline P. vivax mortality & $\begin{array}{l}0.0001 \text { (95\% CI: } \\
0-0.001)\end{array}$ & Beta $(0.09,929.01)$ & Assumption \\
\hline Life expectancy for males aged 20-24 in Myanmar & $48.9 \pm 20 \%$ & Gamma $(96.79,0.51)$ & {$[38]$} \\
\hline Life expectancy for females aged 20-24 in Myanmar & $52 \pm 20 \%$ & Gamma $(96.79,0.54)$ & {$[38]$} \\
\hline Length disability: clinical malaria & $0.008(0.003-0.019)$ & Beta $(3.27,404.86)$ & 3 days (range $1-7$ ) \\
\hline $\begin{array}{l}\text { Length disability: moderate anaemia due to clinical } \\
\text { malaria }\end{array}$ & $0.083(0.04-0.17)$ & Beta $(5.39,59.33)$ & [39] 1 month (range $0.5-2$ ) \\
\hline Length disability: severe malaria & $0.019(0.008-0.027)$ & Beta $(16.91,872.94)$ & 7 days (range $3-10$ ) \\
\hline $\begin{array}{l}\text { Length disability: severe anaemia due to severe } \\
\text { malaria or haemolysis }\end{array}$ & $0.250(0.083-0.500)$ & Beta $(3.59,10.76)$ & 3 months (range 1-6) \\
\hline Disability weight for clinical malaria & $0.053(0.033-0.081)$ & $\begin{array}{l}\text { Gamma }(18.12, \\
0.003)\end{array}$ & [40] infectious disease: moderate acute episode \\
\hline $\begin{array}{l}\text { Disability weight for moderate anaemia due to } \\
\text { clinical malaria }\end{array}$ & $0.058(0.038-0.086)$ & $\begin{array}{l}\text { Gamma }(21.70, \\
0.003)\end{array}$ & {$[40]$} \\
\hline Disability weight for severe malaria & $0.210(0.139-0.298)$ & $\begin{array}{l}\text { Gamma }(26.50, \\
0.008)\end{array}$ & [40] infectious disease: severe acute episode \\
\hline $\begin{array}{l}\text { Disability weight for severe anaemia due to severe } \\
\text { malaria or haemolysis }\end{array}$ & $0.164(0.112-0.228)$ & $\begin{array}{l}\text { Gamma }(30.37, \\
0.005)\end{array}$ & {$[40]$} \\
\hline
\end{tabular}

* Parameters: Beta (alpha, beta), Lognormal (log (mean), log (standard deviation)) and Gamma (shape, scale) 
appropriate for lower income countries [42]; consequently, a threshold of US\$500 was chosen to reflect the resource limitations of healthcare facilities serving migrant and refugee communities.

\subsubsection{Sensitivity analyses}

A one-way sensitivity analysis was conducted to examine the impact of parameter values on the overall outcome. Low and high values were taken from 95\% CIs when available. When not available, the point estimate was varied by $50 \%$ and given wider intervals when necessary to reflect the uncertainty (Table 4.2). Results that varied from the base case by more than US $\$ 0.05$ or 0.0002 DALYs averted were reported. A probabilistic sensitivity analysis (PSA) was conducted to incorporate the uncertainty of all parameters over 1000 sampling iterations using the parameter ranges used in the one-way sensitivity analysis. Table 4.2 lists the distributions used in the PSA. The sum of squared differences was minimised from the specified ranges to produce the shape values for the beta and gamma distributions and random numbers were generated from these distributions. The mean number of recurrences for each iteration was calculated from 100 bootstrapped data points that were randomly sampled from the data set with replacement [24]. The PSA produced a mean estimate and $95 \%$ credible intervals (CrIs) for the costs, DALYs and incremental results.

A key concern is adherence to primaquine regimens by the patients as well as compliance to guidelines by prescribers, which is collectively referred to as adherence here. In order to account for this, a two-way sensitivity analysis examined the interplay of costs and benefits depending on adherence to the primaquine strategy (whether primaquine was administered to the patient and the full course taken) and screening strategy (whether a G6PD RDT plus primaquine was administered to the patient and the full course taken). This cohort analysis assumed that at $0 \%$ adherence all individuals have a relative risk and mean number of recurrences equivalent to receiving chloroquine only. The proportion of individuals in the population who are adherent increases steadily until 100\% adherence, which assumes that recurrences are equivalent 
to the base case. Costs of supervised primaquine and G6PD screening were also varied accordingly. Assumptions about adherence in individuals with G6PD deficiency who receive 14 day primaquine remain the same as the base case analysis.

\subsection{Results}

\subsubsection{Comparison with the chloroquine strategy}

Costs and DALYs for each strategy are shown in Table 4.3 and the cohort results are in Table 4.4. On the Thailand-Myanmar border, the screening strategy averted more DALYs than the chloroquine strategy: 0.026 for males and 0.024 for females. These gains were produced for similar costs. The base case ICERs were US $\$ 6.3$ and US $\$ 11.7$ per DALY averted for males and females respectively.

Figure 4.3 shows the results of the one-way sensitivity analysis. The screening strategy always averted more DALYs than the chloroquine strategy (Figure 4.3C-D). Costs for the screening strategy were highest when radical cure had a low impact on recurrences, when the costs of supervised therapy and the G6PD RDT were increased, and also when the cost of a recurrence was decreased (Figure 4.3A-B). The only assumptions that made the screening strategy cost over US\$500 per DALY averted were lowering the number of recurrences after chloroquine to one (US\$3,678.7 in males and US\$3,724.5 in females) and assuming the same relative risk of having at least one recurrence in females (US\$1,223.6).

The mean costs and DALYs and CrIs estimated by the PSA are shown in Table 4.3. The screening strategy cost more than the chloroquine strategy with mean incremental cost of US\$0.8 (95\%CrI: 17.4 to 19.7) and 0.026 DALYs averted (95\%CrI: 0.007 to 0.117) per male (Figure 4.4A). At a WTP threshold of US\$500, the screening strategy had an $81.2 \%$ probability of being cost-effective (Figure $4.5 \mathrm{~A}$ ). The PSA resulted in a mean incremental cost of US\$0.75 (95\%CrI: 15.0 to 20.0) with 0.023 DALYs averted (95\%CrI: 0.006 to 0.122 ) per female (Figure $4.4 \mathrm{C}$ ) and a $77.6 \%$ probability of being cost-effective at a WTP threshold of US $\$ 500$ (Figure 4.5C). The ICERs were US $\$ 31.3$ 
A) Base case incremental costs $=\$ 0.2$

Number of recurrences after chlorquine treatment Relative risk of having at least 1 recurrence if given primaquine Cost of observed therapy Cost of P. vivax visit Cost of G6PD RDT Cost of severe $P$. vivax recurrence Proportion of $P$. vivax cases that are severe Number of recurrences after chloroquine and primaquine treatment RDT specificity Cost of primaquine pills Proportion severe G6PD abnormal

$$
\begin{array}{r}
\text { RDT sensitivity } \\
\text { P. vivax mortality }
\end{array}
$$

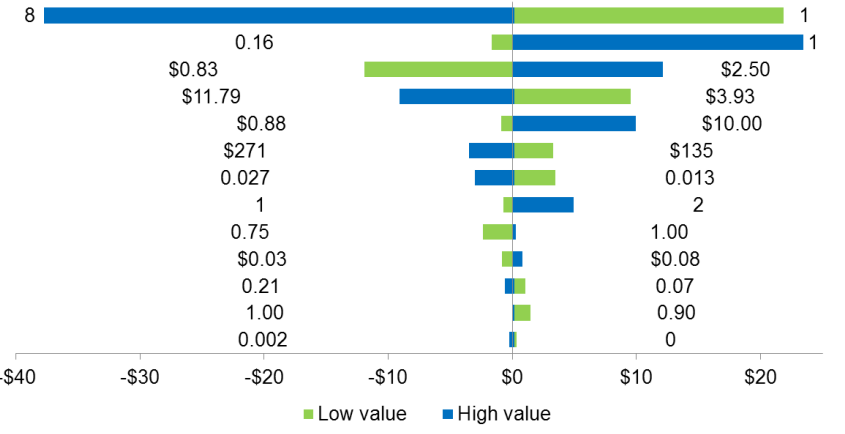

B) Base case incremental costs $=\$ 0.3$

Number of recurrences after chlorquine treatment Relative risk of having at least 1 recurrence if given primaquine Cost of observed therapy Cost of P. vivax recurrence Cost of severe $P$. vivax recurrence Cost of severe $P$. vivax recurrence
Proportion of $P$. vivax cases that are severe Number of recurrences after chloroquine and primaquine treatment RDT specificity Cost of primaquine pills Proportion of moderate G6PD abnormal needing blood transfusion RDT sensitivity for intermediate G6PD abnormal Proportion moderate G6PD abnormal RDT sensitivity for severe G6PD abnormal Cost of hemolytic episode requiring transfusion

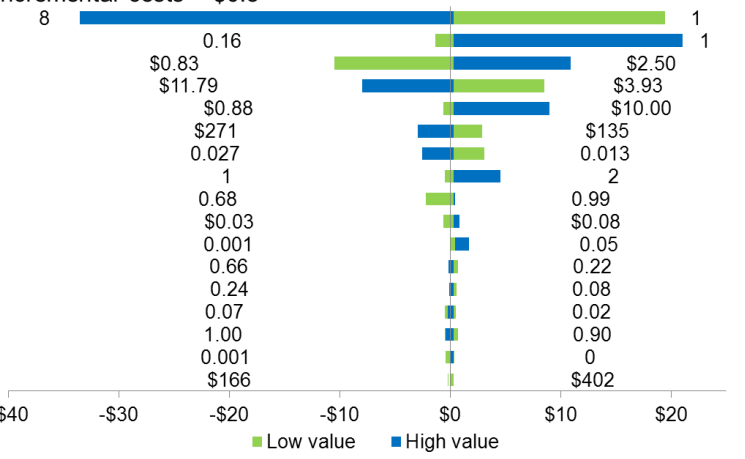

C) Base case DALYs averted $=0.026$ P. vivax mortality Number of recurrences after chlorquine treatment Length of disability for moderate anemia (due to clinical P. vivax) DALY weight for moderate anemia (due to clinical P. vivax) Relative risk of having at least 1 recurrence if given primaquine Life expectancy Length of disability for severe anemia Length of disability for clinical $P$. vivax Number of recurrences after chloroquine and primaquine treatment DALY weight for severe anemia (due to severe P. vivax or severe hemolysis) Proportion of P. vivax cases that are severe
RDT sensitivity DALY weight for P. vivax

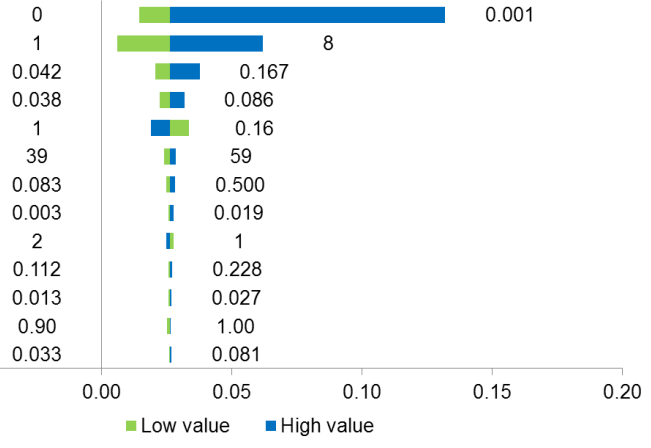

D) Base case DALYs averted $=0.024$ P. vivax mortality Number of recurrences after chlorquine treatment Length of disability for moderate anemia (due to clinical P. vivax) DALY weight for moderate anemia (due to clinical P. vivax) Relative risk of having at least 1 recurrence if given primaquine Life expectancy Proportion who are pregnan ength of disability for severe anemia Proportion of moderate G6PD abnormal needing blood transfusion
Length of disability for clinical P. vivax
Number of recurrences after chloroquine and primaquine treatment Number of recurrences after chloroquine and primaquine treatment DALY weight for severe anemia (due to severe $P$. vivax or severe haemolysis) Proportion of $P$. vivax cases that are severe DALY weight for $P$. vivax Mortality due to not receiving a transfusion
RDT sensitivity for severe G6PD abnormal Proportion not receiving a needed blood transfusion

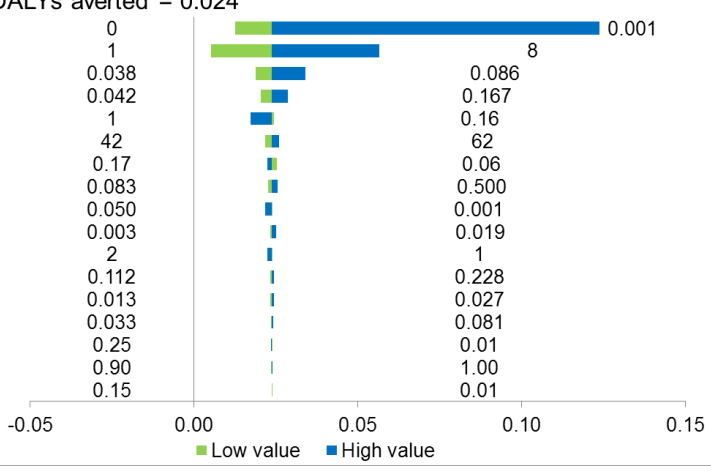

Figure 4.3: One-way sensitivity analysis results on costs and DALYs for the comparison between the screening strategy and the chloroquine strategy for males (A \& C) and females (B \& D). The ranges used are specified in Table 4.2. 
Table 4.3: Costs and DALY results over one year per primary episode treated in the base case analysis and PSA. All costs are in 2014 US\$.

\begin{tabular}{lrrrr}
\hline & \multicolumn{2}{c}{ Mean costs } & \multicolumn{2}{c}{ Mean DALYs } \\
& Base case & PSA $\left(95 \%\right.$ CrI$\left.^{*}\right)$ & Base case & PSA $\left(95 \%\right.$ CrI$\left.^{*}\right)$ \\
\hline Males & & & & \\
Chloroquine strategy & 38.0 & $37.8(24.6-55.5)$ & 0.034 & $0.034(0.009-0.137)$ \\
Primaquine strategy & 45.3 & $45.8(31.6-62.6)$ & 0.019 & $0.019(0.007-0.050)$ \\
Screening strategy & 38.2 & $38.6(25.2-55.3)$ & 0.007 & $0.008(0.003-0.018)$ \\
Females & & & & \\
Chloroquine strategy & 38.0 & $38.3(24.0-54.4)$ & 0.034 & $0.034(0.009-0.155)$ \\
Primaquine strategy & 40.5 & $41.2(28.3-59.0)$ & 0.015 & $0.015(0.006-0.040)$ \\
Screening strategy & 38.3 & $39.1(25.6-57.3)$ & 0.011 & $0.011(0.004-0.030)$ \\
\hline
\end{tabular}

* Credible interval.

per DALY averted for males and US\$32.4 for females.

\subsubsection{Comparison with the primaquine strategy}

Again, the screening strategy resulted in better health outcomes in the base case with 0.011 DALYs averted in males and 0.004 in females (Table 4.3). The health gains in females were more modest due to their overall lower probability of haemolysis requiring transfusion. In addition, the screening strategy produced cost savings of US\$7.1 and US\$2.2 per male and female initially treated, respectively (Table 4.3). The simulation output indicated that one death due to haemolysis would be expected for every 6,682 males and 15,994 non-pregnant females treated using the primaquine strategy. This would be reduced to one death per 668,164 males and 201,198 non-pregnant females treated with the screening strategy (Table 4.4).

The one-way sensitivity analysis showed that changes in the parameter values had a smaller impact on the costs when comparing screening and primaquine strategies, especially for females, and results that consistently averted DALYs (Figure 4.6). The parameters related to mortality, the need for transfusion and the prevalence of G6PD deficiency had the highest impact on DALY results (Figure 4.6A-B). The screening strategy was cost saving with the exception of raising the G6PD RDT cost to US\$10.0, 
Screening verses Chloroquine
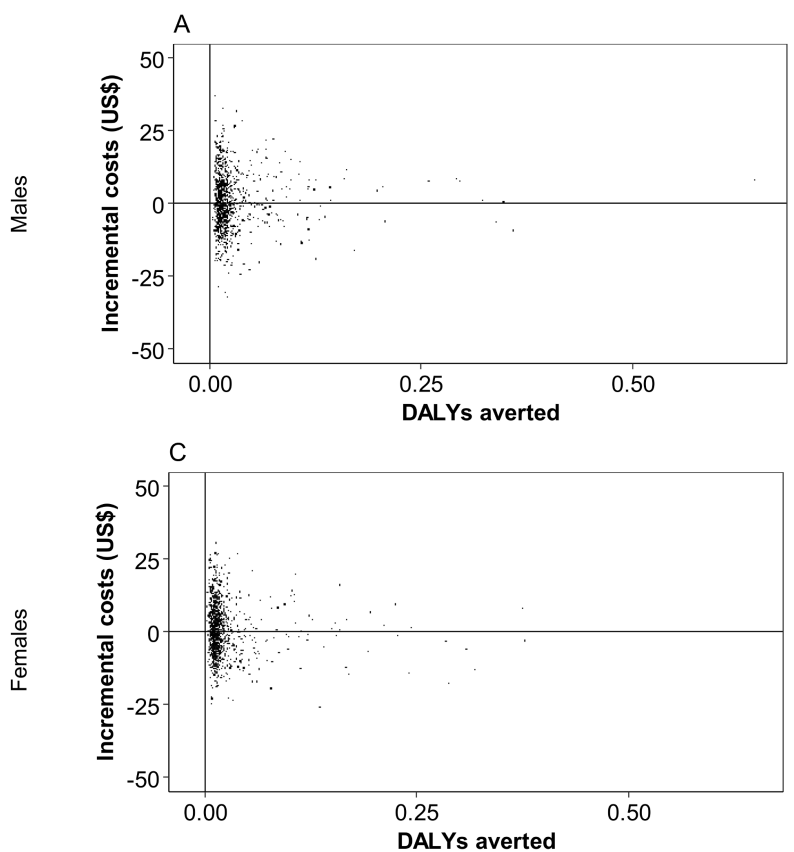

Screening verses Primaquine
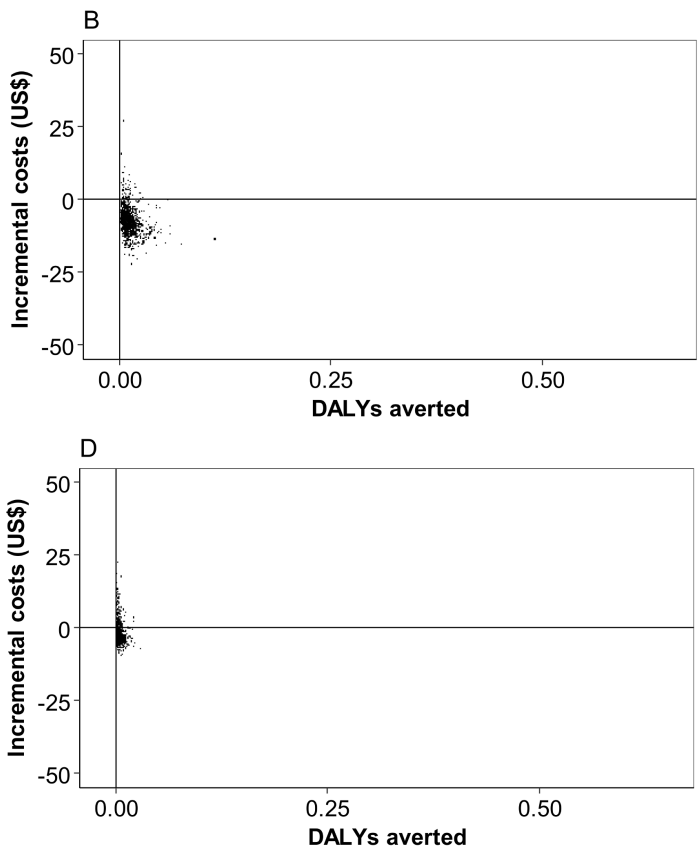

Figure 4.4: Cost-effectiveness plane showing the incremental costs and DALYs averted from the PSA. Results show the screening strategy versus: A) the chloroquine strategy in males, B) the primaquine strategy in males, C) the chloroquine strategy in females and D) the primaquine strategy in females.

Screening verses Chloroquine
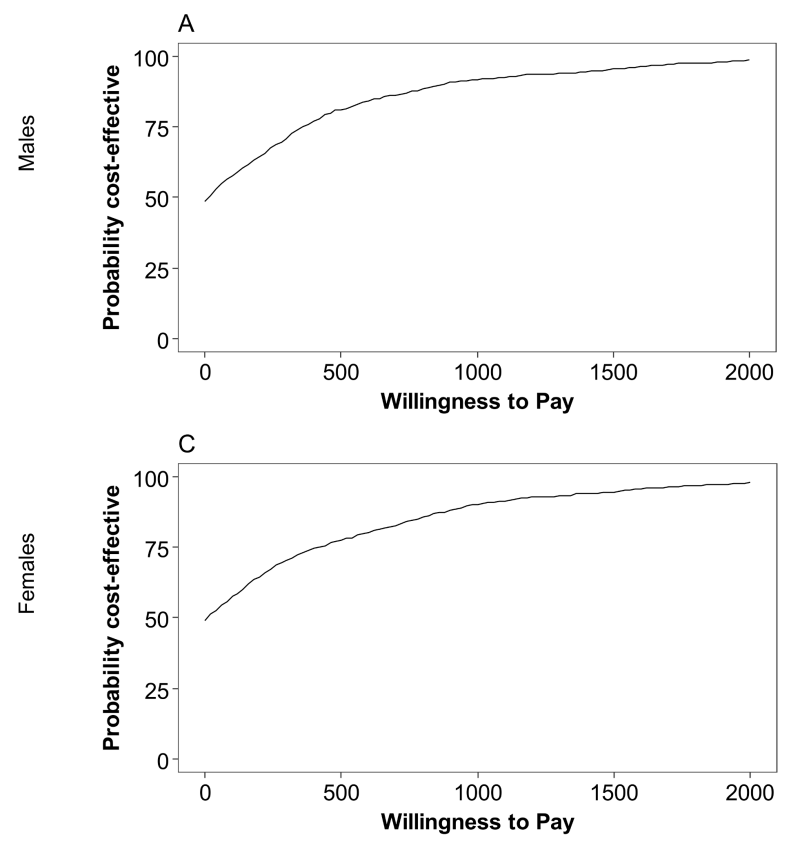

Screening verses Primaquine
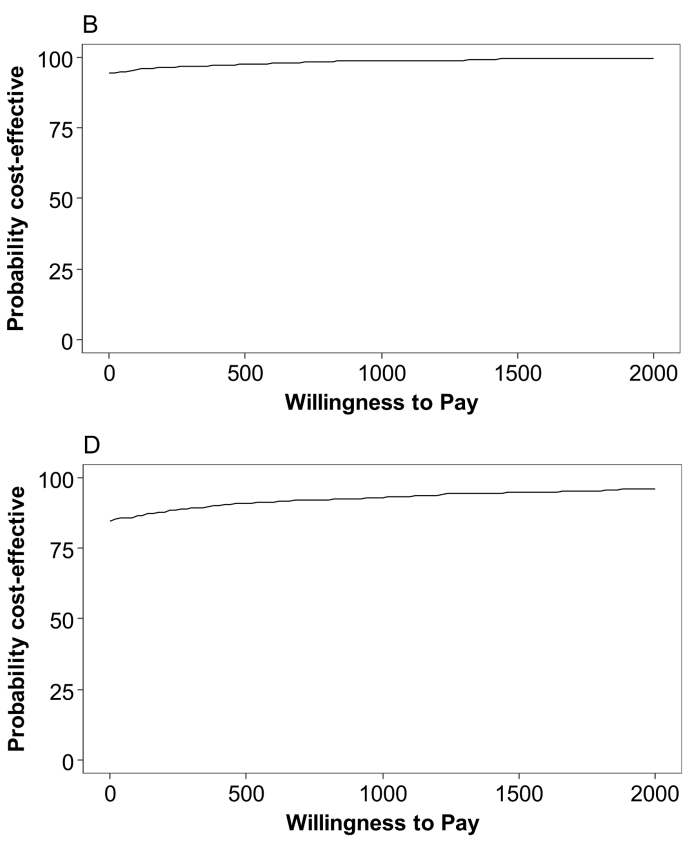

Figure 4.5: Cost-effectiveness acceptability curves. Results show the screening strategy versus: A) the chloroquine strategy in males, B) the primaquine strategy in males, C) the chloroquine strategy in females and D) the primaquine strategy in females. 
Table 4.4: The table shows simulated outcomes for 1,000 P. vivax malaria patients of undetermined G6PD status at attendance, if they were managed according to each of three strategies (chloroquine, primaquine and screening) according to G6PD status, treatment given and the test result if screened.

\begin{tabular}{|c|c|c|c|c|c|c|}
\hline \multirow[b]{3}{*}{ Initial treatment by G6PD status } & \multicolumn{3}{|c|}{ Males } & \multicolumn{3}{|c|}{ Females } \\
\hline & \multicolumn{3}{|c|}{ Strategy } & \multicolumn{3}{|c|}{ Strategy } \\
\hline & Chloroquine & Primaquine & Screening & Chloroquine & Primaquine & Screening \\
\hline G6PD normal who get primaquine and have no recurrences & - & 725 & 718 & - & 592 & 592 \\
\hline G6PD normal who get primaquine and have at least one recurrence & - & 138 & 137 & - & 113 & 113 \\
\hline G6PD normal who get chloroquine only and have no recurrences & 231 & - & 2 & 212 & - & - \\
\hline G6PD normal who get chloroquine only and have at least one recurrence & 632 & - & 6 & 580 & - & - \\
\hline G6PD deficients* who get chloroquine only & 137 & - & - & 208 & - & - \\
\hline G6PD deficients* who get weekly primaquine & - & - & 136 & - & - & 106 \\
\hline Severe G6PD deficients who get 14 day primaquine, do not complete treatment but do not require a transfusion & - & 122 & 1 & - & 40 & 0.40 \\
\hline Intermediate G6PD deficients who get 14 day primaquine and complete treatment & - & - & - & - & 140 & 78 \\
\hline G6PD deficients* who get primaquine, haemolyse and receive a required transfusion & - & 13 & 0.13 & - & 5 & 0.40 \\
\hline G6PD deficients* who get primaquine, haemolyse, do not receive a required transfusion and live & - & 1 & 0.01 & - & 0.50 & 0.04 \\
\hline G6PD deficients* who get primaquine, haemolyse and die due to not receiving a transfusion & - & 0.15 & 0.001 & - & 0.06 & 0.004 \\
\hline Do not receive primaquine due to pregnancy & - & - & - & - & 110 & 110 \\
\hline
\end{tabular}

* For women, this includes both severe and intermediate G6PD deficiency. 


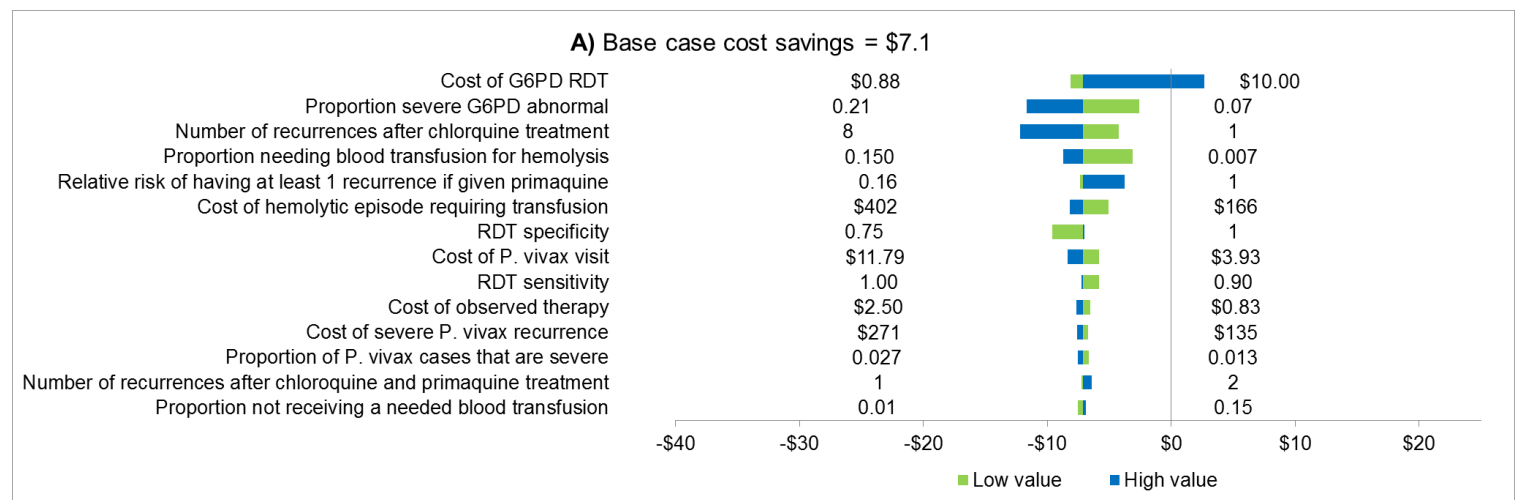

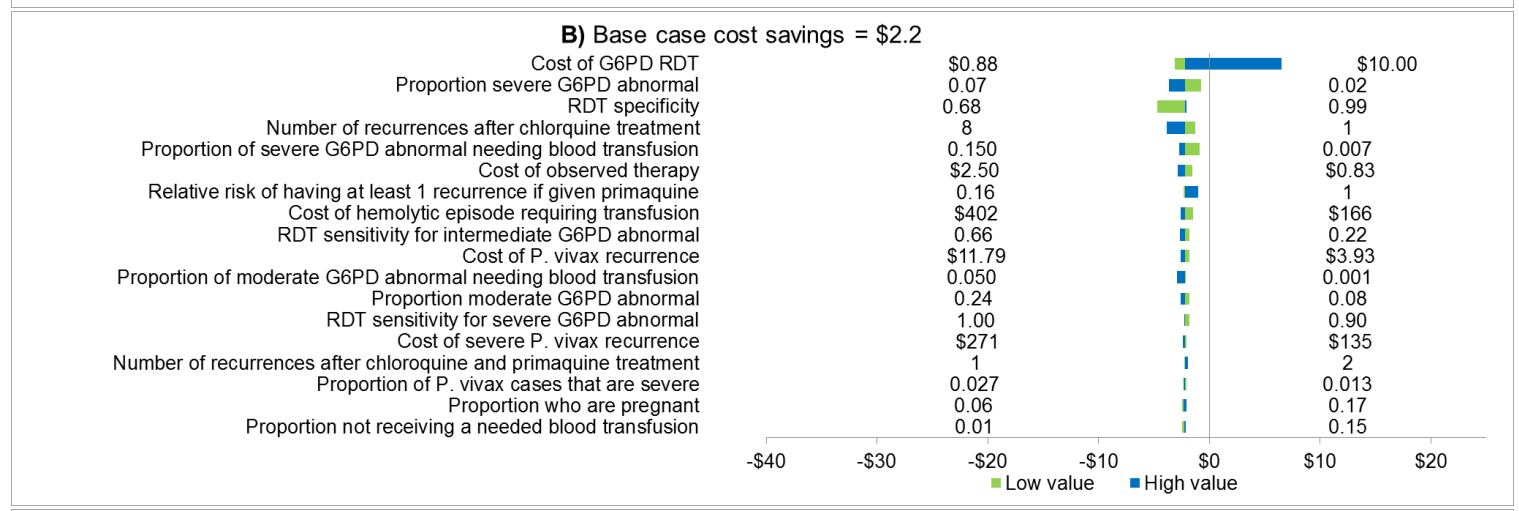

C) Base case DALYs averted = 0.011
P. vivax mortality
Proportion severe G6PD abnormal
Proportion needing blood transfusion for hemolysis
Proportion not receiving a needed blood transfusion
Number of recurrences after chlorquine treatment
Life expectancy

\begin{tabular}{|c|c|c|c|c|c|}
\hline \multicolumn{6}{|c|}{ D) Base case DALYs averted $=0.004$} \\
\hline Mortality due to not receiving a transfusion & 0.01 & $\mid \mathbf{\square}$ & & 0.25 & \\
\hline P. vivax mortality & 0 & $\overline{\mathbf{a}}$ & & 0.001 & \\
\hline Proportion severe G6PD abnormal & 0.02 & 1 & & 0.07 & \\
\hline Proportion not receiving a needed blood transfusion & 0.01 & I & & 0.15 & \\
\hline Proportion of severe G6PD abnormal needing blood transfusion & 0.007 & I & & 0.150 & \\
\hline Number of recurrences after chlorquine treatment & 1 & I & & 8 & \\
\hline Proportion of moderate G6PD abnormal needing blood transfusion & 0.001 & I & & 0.050 & \\
\hline Life expectancy & 42 & I & & 62 & \\
\hline Length of disability for moderate anemia (due to clinical P. vivax) & 0.038 & 1 & & 0.086 & \\
\hline $\begin{array}{ll}\text { Proportion who are pregnant } \\
\end{array}$ & 0.17 & 1 & & 0.06 & \\
\hline Length of disability for severe anemia & 0.083 & 1 & & 0.500 & \\
\hline DALY weight for moderate anemia (due to clinical $P$. vivax) & 0.042 & 1 & & 0.167 & \\
\hline RDT sensitivity for severe G6PD abnormal & 0.90 & 1 & & 1.00 & \\
\hline \multirow[t]{2}{*}{ Relative risk of having at least 1 recurrence if given primaquine } & 1 & 1 & & 0.16 & \\
\hline & -0.05 & 0.00 & $\begin{array}{c}0.05 \\
\text { Low value }\end{array}$ & $\begin{array}{r}0.10 \\
\text { = High value }\end{array}$ & 0.15 \\
\hline
\end{tabular}

Figure 4.6: One-way sensitivity analysis results on costs and DALYs for the comparison between the screening strategy and the chloroquine strategy for males (A \& C) and females (B \& D). The ranges used are specified in Table 4.2. 
which caused an incremental cost for the screening strategy of US $\$ 235.9$ and US $\$ 1,602.1$ for males and females, respectively (Figure 4.6A-B). The screening strategy remained cost saving even at low levels of G6PD deficiency (7\%) (Figure 4.6A-B). The PSA showed a mean cost savings of US $\$ 7.3$ (95\%CrI: -15.4 to 3.4$)$ and 0.012 DALYs averted (95\%CrI: 0.001 to 0.113 ) in males (Figure 4.4B), and a mean cost savings of US\$2.2 (95\%CrI: 6.2 to 6.7 ) and 0.004 DALYs averted (95\%CrI: 0.000 to 0.029) in females (Figure 4.4D). The screening strategy had a $97.7 \%$ probability of being cost-effective for males at a WTP threshold of US $\$ 500.0$ (Figure 4.5B). For females, the probability was $91.1 \%$ (Figure $4.5 \mathrm{D}$ ). Table 4.3 shows the cost and DALY estimates from the PSA.

The two-way analysis (Figure 4.7) demonstrated that the screening strategy would be cost-effective in scenarios where it is used to maintain or increase the number of patients who are adherent to their primaquine regimens. The impact of switching to the screening strategy was slightly less in females due to the exclusion of pregnant women from primaquine treatment and the low sensitivity of the G6PD RDT in women with intermediate G6PD deficiency.

\subsubsection{Web-based application}

Due to the extensive heterogeneity and parameter uncertainty around key parameter estimates, notably relapse patterns [27], G6PD variants and prevalence [10] and costs, a web-based interface was built using the R-Shiny application so that the model could be adapted to other settings as need be. See website

(https://malaria.shinyapps.io/g6pd_screening/).

\subsection{Discussion}

Point-of-care G6PD RDTs offer the opportunity for the safe uptake of primaquine for the prevention of recurrences. Our findings suggest that on the Thailand-Myanmar border the use of G6PD RDTs to identify patients with G6PD deficiency before 
A

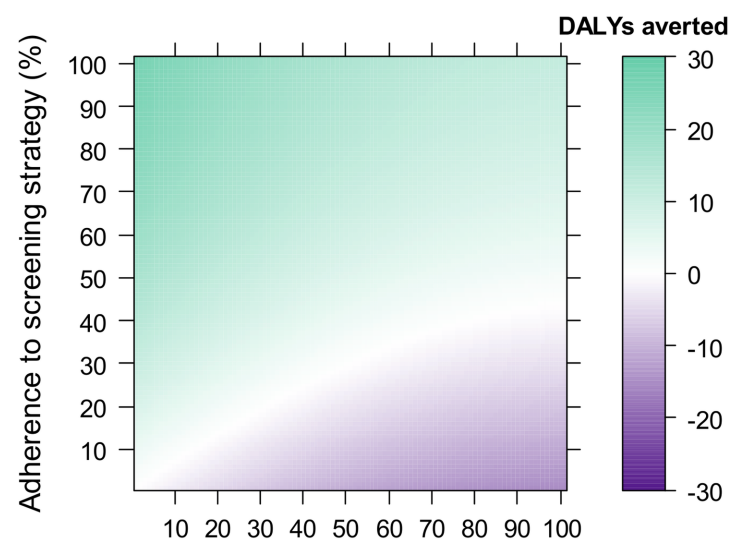

Adherence to primaquine strategy (\%)
B

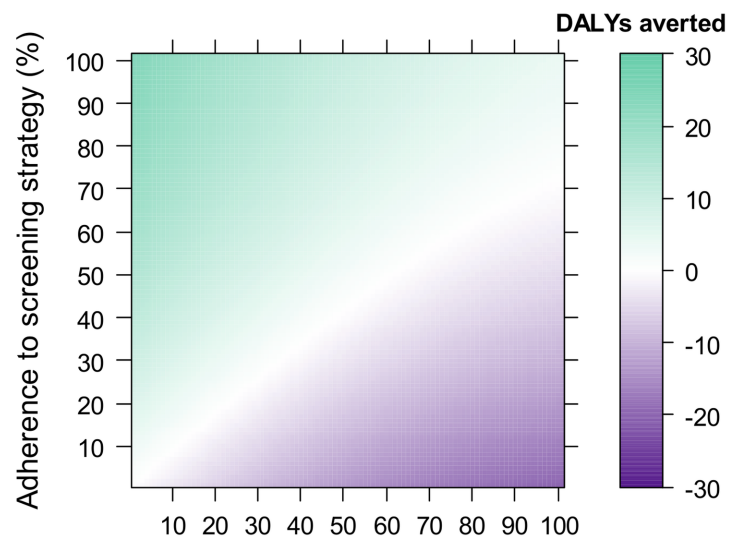

Adherence to primaquine strategy (\%)
C

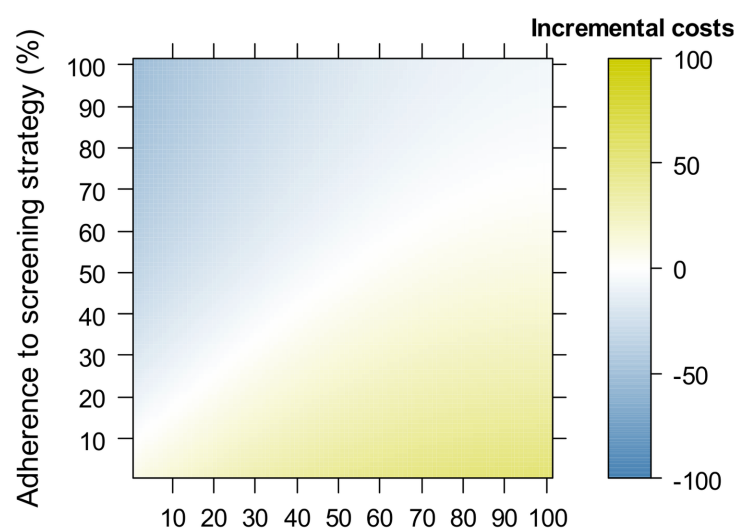

Adherence to primaquine strategy (\%)

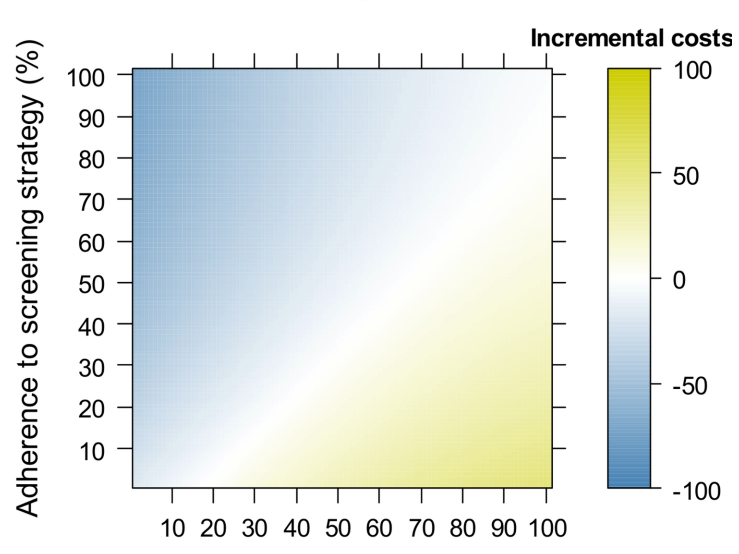

Adherence to primaquine strategy (\%)
E

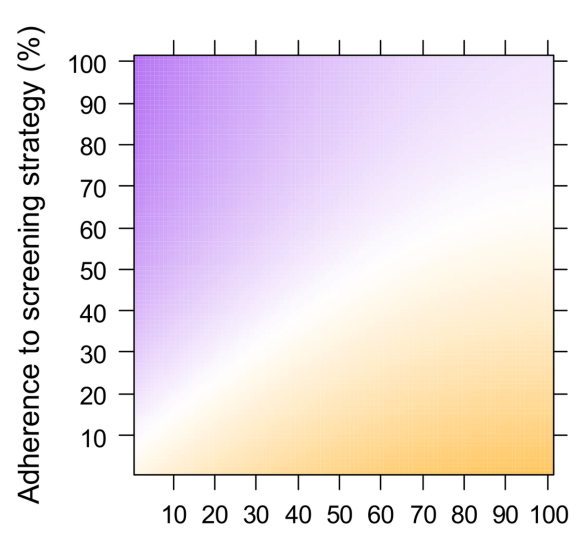

Adherence to primaquine strategy (\%)

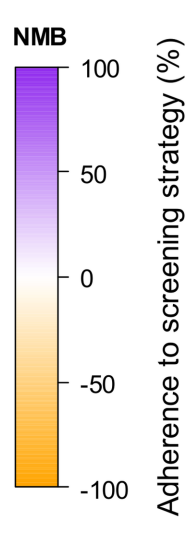

$\mathbf{F}$

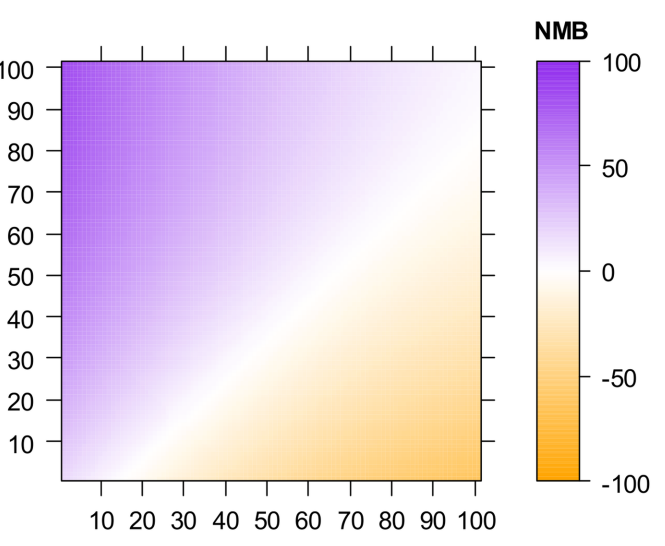

Adherence to primaquine strategy (\%)

Figure 4.7: Two-way sensitivity analysis results showing the impact of changes in the level of adherence to primaquine regimens. The left column is for males and the right column is for females. Green represents DALYs averted by the screening strategy for a cohort of 1,000 individuals. For costs, yellow indicates increased costs and blue indicates cost savings for the screening strategy for one individual. For net monetary benefit (NMB), purple indicates scenarios where the screening strategy would be cost-effective at a threshold of US\$500 $(T)$ where $N M B=T \times \triangle D A L Y s-\triangle$ Costs. 
supervised primaquine is likely to provide significant health benefits (equivalent to between one and nine days of perfect health) compared to giving chloroquine alone or giving 14 day primaquine without G6PD testing. Furthermore, the use of point-of-care G6PD RDTs will potentially save costs or, at most, increase them moderately. While policy decisions primarily impact the public sector, it is hoped that the effect could spillover to the private sector as seen in Chapter 3. If the population was aware of the importance of both radical cure to prevent future relapses and of G6PD screening to prevent unnecessary side effects, then the demand for these things could be sufficient to incentivise the private sector to implement a similar policy.

Primaquine is currently the only licensed hypnozonticidal drug, but healthcare professionals who treat $P$. vivax cases are often more concerned with avoiding the immediate risk of haemolysis than with protecting the patient from the risks associated with future relapses. The fear of haemolysis incentivises healthcare providers to avoid using primaquine. In other settings, primaquine may be administered without G6PD testing, putting individuals with G6PD deficiency at risk of severe haemolysis, although the degree of risk will depend upon local G6PD variants and their prevalence. The providers in these settings may not implement G6PD screening due to the perception that the risk of haemolysis is too low to justify the use of G6PD tests or they may be motivated by the short-term cost savings from not screening for G6PD deficiency. In the scenario presented, the use of G6PD RDTs will save costs while averting DALYs compared to a policy in which primaquine is administered without G6PD testing. While our results give a high probability of cost savings when switching from the primaquine strategy to the screening strategy, this should not deter radical cure without screening in settings where screening is unavailable as the primaquine strategy averted more DALYs than the chloroquine strategy.

Our model is based on supervised primaquine therapy aned hence our findings may not be applicable to other settings where unsupervised primaquine is the norm and adherence to a complete course of treatment and thus effectiveness may be low [43]. Patients feel better after the first few days of treatment, giving them little incentive to 
complete their primaquine regimen unless educated about its importance in preventing future $P$. vivax episodes. While the assumption that adherence is perfect is ambitious, it was necessary to reflect the clinical trial outcomes, which were the result of supervised treatment. Future research investigating the relationship between adherence to primaquine therapy and effectiveness would enable better cost-effectiveness studies. The two-way analysis on adherence (Figure 4.7) enabled comparison between settings with varying adherence and how this impacts upon cost effectiveness. The screening strategy averts more DALYs than the primaquine strategy, even at relatively high primaquine strategy adherence and low screening strategy adherence. Shorter drug courses, such as seven day primaquine and tafenoquine, should contribute to higher adherence levels and reduced costs for the primaquine and screening strategies. Other options include but are not limited to text message reminders, observed therapy by family members, and increased education of health care workers and patients on the importance of adherence.

Overall, the screening strategy was less cost-effective in women as compared to men. This reflects a greater proportion of women who are excluded from receiving primaquine due to pregnancy and the lower risk of severe haemolysis in females with intermediate G6PD deficiency. Since men represented $65 \%$ of patients in the trial that the recurrence data were derived from [24], the overall cost-effectiveness estimates per person presenting with $P$. vivax malaria would likely be closer to the results for males. The cost-effectiveness of using G6PD RDTs is also dependent on the diagnostic accuracy of the test. Our model draws on studies conducted on the Thailand-Myanmar border, which demonstrated a high sensitivity in healthy volunteers. While other studies have shown similar results $[16,17]$ in healthy volunteers, a recent study in Brazil found that the sensitivity of the CareStart G6PD RDT dropped to $50 \%$ in patients with malaria compared to $80 \%$ in those who did not [15]. A recent cost-effectiveness analysis of male patients with P. vivax malaria in Brazil used a low sensitivity for the CareStart G6PD RDT (46\%) but still found it to be more cost-effective than both the BinaxNOW test and routine care; where the analysis also 
involved the prescription of primaquine without having a G6PD test [44]. This study, however, used the endpoints 'adequately diagnosed case' and 'hospitalisation avoided' instead of DALYs. The Brazilian population was given a $94 \%$ probability of hospitalisation when primaquine was given to G6PD deficient men. Our model differs in that we assume a lower rate of hospitalisation due to severe haemolysis. We also include results for both genders and report DALYs, enabling comparisons with interventions for other diseases.

Our study has a number of limitations, mostly related to the model assumptions. The cost-effectiveness of the screening strategy would be increased if it included the onward transmission of $P$. vivax, societal costs, or the longer term impact of repeated episodes, such as anaemia, malnutrition and all-cause mortality. This is particularly relevant in areas such as the Thailand-Myanmar border where the estimated proportion of recurrences due to relapses is estimated to be $78 \%$ [24]. The cost-effectiveness may decrease if some individuals were not able to metabolise primaquine, if healthcare workers were not able to utilise G6PD RDTs or supervise primaquine regimens, if the prevalence of G6PD deficiency in those presenting with $P$. vivax was lower than that in the general population, if the diagnostic accuracy of the G6PD test were lower, if healthcare facilities providing care for haemolytic episodes were not accessible or if the operational costs of implementing a switch to the screening strategy were included. These parameter limitations are similar to those highlighted in a recent review of the costs and cost-effectiveness of $P$. vivax control and elimination [45].

Finally, the model is limited by the paucity of data available in the literature for some parameter values, including the mortality rate for those who have a primaquine-induced haemolytic episode requiring transfusion but do not receive them. These were influential parameters in the comparison between the screening strategy and the primaquine strategy. Our assumptions of primaquine induced mortality were derived from previous risks of mortality in patients treated with dapsone in Africa and equated to a population risk of one in 6,682 administrations to males and one in 15,994 administrations in females. These risks are significantly higher than the risks 
documented in a previous review [11] but the screening strategy averted more DALYs than the primaquine strategy at a lower level of primaquine-induced mortality, though this is likely due to the utilisation of weekly primaquine by the screening strategy. In order to reflect this uncertainty, wide distributions were used in the sensitivity analyses for parameters that were considered to be less reliable. Other variables, such as the prevalence of G6PD deficiency, will vary greatly depending on the epidemiological setting. Unit costs were not collected alongside the trial, requiring cost data to be gathered from various sources. This is a limitation, particularly as the one-way sensitivity analysis showed that the cost parameters had a large influence on the results. The online model (https://malaria.shinyapps.io/g6pd_screening/) could be used to investigate the results when more robust cost data are available. Whilst it would be beneficial to gather more robust parameter estimates on which to base informed policy decisions, this should be tempered by the feasibility of gathering such data and the potential benefits of implementing appropriate policies sooner, especially in the context of elimination.

Although our model is relatively simple, it provides a useful starting point for policymakers to compare the risks and benefits of using G6PD RDTs to enable the safe and effective use of primaquine. To assist in this process, an online tool is provided (https://malaria.shinyapps.io/g6pd_screening/). This model enables policymakers and healthcare providers the opportunity to vary the assumptions made in the model to reflect their local scenario. In this way, decision makers can begin to think about whether the model might be cost-effective in their setting. While every attempt has been made to keep a plausible range of settings, it may be necessary for further consultation with a health economist before implementing policy decisions. In addition, the model can be adjusted to reflect any additional data as it becomes available. A new online tool may need to be developed if new screening and treatment options are available.

As the only licensed antimalarial for the radical cure of $P$. vivax infections, primaquine will be a critical tool for the elimination of all malaria [46] and for the health gains 
provided to patients. The currently available G6PD RDTs can identify G6PD deficient males, making the screening strategy an attractive option regardless of current practice. In situations where blood transfusions are not accessible, further information may be required on the prevalence of G6PD deficiency and associated risk of haemolysis in females with intermediate G6PD deficiency who test normal by current G6PD RDT methods. Despite the initial cost, point-of-care RDTs avert DALYs by reducing recurrences while diminishing the haemolytic risk in G6PD deficient patients. 


\subsection{Appendix: Study description for determining G6PD activity in females}

\subsubsection{Study population and samples}

Healthy women were enrolled over a four month time period as part of an ongoing project by the SMRU among the female migrant and refugee population living at the Thailand-Myanmar border with a target enrolment of 300. No exclusion criteria specific to G6PD status were applied; therefore, the sample is believed to represent the general female population of the area. A venous blood sample was taken from each participant and transported within eight hours to the central laboratory for analyses.

\subsubsection{Laboratory methods}

Screening for G6PD deficiency using the FST (R\&D Diagnostic, Greece). Five microliters of blood were mixed with reagents, spotted on filter paper after 10 minutes of incubation and allowed to air-dry over 20 minutes. Spots were visualized under ultraviolet light and normal or deficient phenotype assigned based on fluorescence. Quantitative phenotype was assessed by spectrophotometry according to WHO protocol on whole blood depleted of white blood cells [47]. G6PD activity was calculated after normalisation with hemoglobin ( $\mathrm{Hb})$ concentration and expressed as percentage of the population median which was previously established using the same technique.

\subsubsection{Statistical analysis}

Three categories of G6PD activity were chosen: $<30 \%, 30-69 \%$ and $\geq 70 \%$. The percentage of women in each of those categories was calculated using $\mathrm{R}$ statistical software [22].

\subsubsection{Results}

322 women were enrolled in the study. Of those who were enrolled, 5.0\% (16/322) had less than $30 \%$ G6PD activity and $15.8 \%(51 / 322)$ tested as having between 30 and $69 \%$ G6PD activity. 


\subsection{Declarations}

\subsubsection{Acknowledgments}

I thank Dr. Ben Cooper for statistical advice.

\subsubsection{Funding}

This study was part of the Wellcome-Trust Major Overseas Programme in SE Asia (grant number 106698/Z/14/Z; www.wellcome.ac.uk) for AD, CSC, GB, FN \& YL. RNP is funded by the Wellcome Trust (grant number 200909; www.wellcome.ac.uk). AD is funded by the Bill \& Melinda Gates Foundation (grant number OPP1054404; www.gatesfoundation.org). The funders had no role in study design, data collection and analysis, decision to publish, or preparation of the manuscript. 


\section{References for Chapter 4}

[1] Price RN, Douglas NM, Anstey NM. New developments in Plasmodium vivax malaria: severe disease and the rise of chloroquine resistance. Curr Opin Infect Dis. 2009;

[2] Rahimi BA, Thakkinstian A, White NJ, Sirivichayakul C, Dondorp AM, Chokejindachai W. Severe vivax malaria: a systematic review and meta-analysis of clinical studies since 1900. Malar J. 2014;13:481. doi:10.1186/1475-2875-13-481.

[3] Carrara VI, Lwin KM, Phyo AP, Ashley E, Wiladphaingern J, Sriprawat K, et al. Malaria burden and artemisinin resistance in the mobile and migrant population on the Thai-Myanmar border, 1999-2011: an observational study. PLoS Med. 2013;10(3):e1001398. doi:10.1371/journal.pmed.1001398.

[4] World Health Organization. World malaria report 2013.; 2013.

[5] Douglas NM, Lampah DA, Kenangalem E, Simpson JA, Poespoprodjo JR, Sugiarto P, et al. Major burden of severe anemia from non-falciparum malaria species in Southern Papua: a hospital-based surveillance study. PLoS Med. 2013;10(12):e1001575; discussion e1001575. doi:10.1371/journal.pmed.1001575.

[6] Douglas NM, Pontororing GJ, Lampah DA, Yeo TW, Kenangalem E, Poespoprodjo JR, et al. Mortality attributable to Plasmodium vivax malaria: a clinical audit from Papua, Indonesia. BMC Med. 2014;12:217. doi:10.1186/s12916-014-0217-z.

[7] Adekunle AI, Pinkevych M, McGready R, Luxemburger C, White LJ, Nosten F, et al. Modeling the dynamics of Plasmodium vivax infection and hypnozoite reactivation in vivo. PLoS Negl Trop Dis. 2015;9(3):e0003595. doi:10.1371/journal.pntd.0003595.

[8] Cappellini MD, Fiorelli G. Glucose-6-phosphate dehydrogenase deficiency. Lancet. 2008;371(9606):64-74. doi:10.1016/s0140-6736(08)60073-2. 
[9] Howes RE, Battle KE, Satyagraha AW, Baird JK, Hay SI. G6PD deficiency: global distribution, genetic variants and primaquine therapy. Adv Parasitol. 2013;81:133-201. doi:10.1016/b978-0-12-407826-0.00004-7.

[10] Howes RE, Piel FB, Patil AP, Nyangiri OA, Gething PW, Dewi M, et al. G6PD deficiency prevalence and estimates of affected populations in malaria endemic countries: a geostatistical model-based map. PLoS Med. 2012;9(11):e1001339. doi:10.1371/journal.pmed.1001339.

[11] Ashley EA, Recht J, White NJ. Primaquine: the risks and the benefits. Malar J. 2014;13:418. doi:10.1186/1475-2875-13-418.

[12] Bassat Q, Velarde M, Mueller I, Lin J, Leslie T, Wongsrichanalai C, et al. Key Knowledge Gaps for Plasmodium vivax Control and Elimination. Am J Trop Med Hyg. 2016;doi:10.4269/ajtmh.16-0180.

[13] World Health Organization. Control and elimination of plasmodium vivax malaria: a technical brief; 2015.

[14] Luzzatto L, Seneca E. G6PD deficiency: a classic example of pharmacogenetics with on-going clinical implications. Br J Haematol. 2014;164(4):469-80. doi:10.1111/bjh.12665.

[15] Brito MA, Peixoto HM, Almeida AC, Oliveira MR, Romero GA, Moura-Neto JP, et al. Validation of the rapid test Carestart ${ }^{\mathrm{TM}}$ G6PD among malaria vivax-infected subjects in the Brazilian Amazon. Rev Soc Bras Med Trop. 2016;49(4):446-55. doi:10.1590/0037-8682-0134-2016.

[16] Baird JK, Dewi M, Subekti D, Elyazar I, Satyagraha AW. Noninferiority of glucose-6-phosphate dehydrogenase deficiency diagnosis by a point-of-care rapid test vs the laboratory fluorescent spot test demonstrated by copper inhibition in normal human red blood cells. Transl Res. 2015;165(6):677-88.

doi:10.1016/j.trsl.2014.09.009. 
[17] Satyagraha AW, Sadhewa A, Elvira R, Elyazar I, Feriandika D, Antonjaya U, et al. Assessment of Point-of-Care Diagnostics for G6PD Deficiency in Malaria Endemic Rural Eastern Indonesia. PLoS Negl Trop Dis. 2016;10(2):e0004457. doi:10.1371/journal.pntd.0004457.

[18] Bancone G, Chu CS, Chowwiwat N, Somsakchaicharoen R, Wilaisrisak P, Charunwatthana $\mathrm{P}$, et al. Suitability of capillary blood for quantitative assessment of G6PD activity and performances of G6PD point-of-care tests. Am J Trop Med Hyg. 2015;92(4):818-24. doi:10.4269/ajtmh.14-0696.

[19] Osorio L, Carter N, Arthur P, Bancone G, Gopalan S, Gupta SK, et al. Performance of BinaxNOW G6PD deficiency point-of-care diagnostic in P. vivaxinfected subjects. Am J Trop Med Hyg. 2015;92(1):22-7. doi:10.4269/ajtmh.14-0298.

[20] Baird JK. Point-of-care G6PD diagnostics for Plasmodium vivax malaria is a clinical and public health urgency. BMC Med. 2015;13(1):296. doi:10.1186/s12916-015-0531-0.

[21] Claxton K, Revill P, Sculpher M, Wilkinson T, Cairns J, Briggs A. The Gates Reference Case for Economic Evaluation. The Bill \& Melinda Gates Foundation; 2014 .

[22] R Core Team. R: A language and environment for statistical computing. 2016;Available from: https://www.R-project.org/.

[23] University of Oxford. Study on the Treatment of Vivax Malaria (VHX). National Library of Medicine (US). 2013;Available from: https://clinicaltrials .gov/ct2/show/NCT01074905?term=efficacy+ chloroquine+artesunate+primaquine\&rank=1 NLM Identifier: NCT01074905(Accessed: 2016 March 15).

[24] Chu CS. Factors affecting Plasmodium vivax recurrence. PhD Thesis, The Open University. 2015; 
[25] John GK, Douglas NM, von Seidlein L, Nosten F, Baird JK, White NJ, et al. Primaquine radical cure of Plasmodium vivax: a critical review of the literature. Malar J. 2012;11:280. doi:10.1186/1475-2875-11-280.

[26] Bancone G, Chu CS, Somsakchaicharoen R, Chowwiwat N, Parker DM, Charunwatthana P, et al. Characterization of G6PD genotypes and phenotypes on the northwestern Thailand-Myanmar border. PLoS One. 2014;9(12):e116063. doi:10.1371/journal.pone.0116063.

[27] Battle KE, Karhunen MS, Bhatt S, Gething PW, Howes RE, Golding N, et al. Geographical variation in Plasmodium vivax relapse. Malar J. 2014;13:144. doi:10.1186/1475-2875-13-144.

[28] World Health Organisation. Guidelines for the Treatment of Malaria. 2015;.

[29] Pamba A, Richardson ND, Carter N, Duparc S, Premji Z, Tiono AB, et al. Clinical spectrum and severity of hemolytic anemia in glucose 6-phosphate dehydrogenase-deficient children receiving dapsone. Blood. 2012;120(20):4123-33. doi:10.1182/blood-2012-03-416032.

[30] Chu CS, Bancone G, Moore KA, Win HH, Thitipanawan N, Po C, et al. Haemolysis in G6PD Heterozygous Females Treated with Primaquine for Plasmodium vivax Malaria: A Nested Cohort in a Trial of Radical Curative Regimens. PLoS Med. 2017;14(2):e1002224. doi:10.1371/journal.pmed.1002224.

[31] Ley B, Luter N, Espino FE, Devine A, Kalnoky M, Lubell Y, et al. The challenges of introducing routine G6PD testing into radical cure: a workshop report. Malaria Journal. 2015;14(1):377.

[32] Lubell Y, Reyburn H, Mbakilwa H, Mwangi R, Chonya K, Whitty CJ, et al. The cost-effectiveness of parasitologic diagnosis for malaria-suspected patients in an era of combination therapy. Am J Trop Med Hyg. 2007;77(6 Suppl):128-32.

[33] MSH (Management Sciences for Health). International Drug Price Indicator Guide, 2014 Edition. 2015; 
[34] Kyaw SS, Drake T, Thi A, Kyaw MP, Hlaing T, Smithuis FM, et al. Malaria community health workers in Myanmar: a cost analysis. Malar J. 2016;15(1):41. doi:10.1186/s12936-016-1102-3.

[35] World Health Organisation. WHO-CHOICE unit cost estimates for service delivery. 2011;Available from: http://www.who.int/choice/ cost-effectiveness/inputs/health_service/en/(Accessed: 02 February 2016).

[36] Kyaw SS, Drake T, Ruangveerayuth R, Chierakul W, White NJ, Newton PN, et al. Cost of treating inpatient falciparum malaria on the Thai-Myanmar border. Malar J. 2014;13:416. doi:10.1186/1475-2875-13-416.

[37] Mulligan JA, Fox-Rushby JA, Adam T, Johns B, Mills A. Unit Costs of Health Care Inputs in Low and Middle Income Regions; 2005.

[38] World Health Organization. type [; 2014]Available from: http://apps. who. int/gho/data/node.main.692?lang=en.

[39] Douglas NM, Anstey NM, Buffet PA, Poespoprodjo JR, Yeo TW, White NJ, et al. The anaemia of Plasmodium vivax malaria. Malar J. 2012;11(1):135. doi:1475-2875-11-135 [pii] 10.1186/1475-2875-11-135.

[40] Salomon JA, Vos T, Hogan DR, Gagnon M, Naghavi M, Mokdad A, et al. Common values in assessing health outcomes from disease and injury: disability weights measurement study for the Global Burden of Disease Study 2010. Lancet. 2013;380(9859):2129-43. doi:10.1016/s0140-6736(12)61680-8.

[41] The World Bank. GDP per capita (current US\$) for Myanmar. 2015;Available from: http:

//data. worldbank . org/indicator/NY . GDP . PCAP. CD?locations=MM(Accessed: 19 August 2016). 
[42] Ochalek J, Lomas J, Claxton K. Cost per DALY averted thresholds for low- and middle-income countries: evidence from cross country data. University of York CHE Research Paper. 2015;.

[43] Bruxvoort K, Goodman C, Kachur SP, Schellenberg D. How patients take malaria treatment: a systematic review of the literature on adherence to antimalarial drugs. PLoS One. 2014;9(1):e84555. doi:10.1371/journal.pone.0084555.

[44] Peixoto HM, Brito MA, Romero GA, Monteiro WM, de Lacerda MV, de Oliveira MR. Cost-effectiveness analysis of rapid diagnostic tests for G6PD deficiency in patients with Plasmodium vivax malaria in the Brazilian Amazon. Malar J. 2016;15(1):82. doi:10.1186/s12936-016-1140-x.

[45] White MT, Yeung S, Patouillard E, Cibulskis R. Costs and Cost-Effectiveness of Plasmodium vivax Control. Am J Trop Med Hyg. 2016;95(6 Suppl):52-61. doi:10.4269/ajtmh.16-0182.

[46] Roy M, Bouma MJ, Ionides EL, Dhiman RC, Pascual M. The potential elimination of Plasmodium vivax malaria by relapse treatment: insights from a transmission model and surveillance data from NW India. PLoS Negl Trop Dis. 2013;7(1):e1979. doi:10.1371/journal.pntd.0001979.

[47] Beutler E, Blume KG, Kaplan JC, Lohr GW, Ramot B, Valentine WN. International Committee for Standardization in Haematology: recommended methods for red-cell enzyme analysis. Br J Haematol. 1977;35(2):331-40. 


\section{Chapter 5}

\section{Economic costs of $P$. vivax}

\section{episodes: A multi-country}

comparative analysis using primary

\section{trial data}

\subsection{Introduction}

Outside of Sub-Saharan Africa, P. vivax is now the dominant species causing malaria with an estimated 12.6 million episodes in 2015 [1]. P. vivax malaria differs from $P$. falciparum in that an infectious bite can result in dormant liver stages of the parasites (hypnozoites) which can relapse months or even years after the primary infection. Hence, one infected mosquito bite can result in multiple clinical episodes, with the risk and frequency of relapses varying by geographical location. The clinical management of a patient with $P$. vivax therefore should involve radical cure with a drug regimen that clears both the blood and liver stages of the parasite.

The only licensed drug with activity against hypnozoites is primaquine, an 8aminoquinoline, which can cause haemolysis when administered to individuals with 
G6PD deficiency. While a single dose of primaquine is used without screening for G6PD deficiency to block P. falciparum transmission, WHO guidelines recommend that all patients should be tested for G6PD deficiency before a high dose of primaquine is prescribed for radical cure of vivax malaria [2]. Few settings routinely offer screening for G6PD deficiency to vivax patients due to operational constraints (Chapter 1), but new point-of-care tests for G6PD deficiency have been developed to facilitate bedside testing. For individuals identified as G6PD deficient, an eight week course of weekly primaquine doses is recommended [3], with monitoring for anaemia, now feasible using a hand held device $\left(\right.$ Haemocue $\left.^{\mathrm{TM}}\right)$. G6PD normal patients with malaria should be treated with a course of primaquine usually prescribed as a once daily regimen for 14 days; however, patient adherence to completing a full treatment course varies by setting and patient population [4] and in practice effectiveness can be very poor [5]. Primaquine efficacy is dependent upon the total $\mathrm{mg} / \mathrm{kg}$ dose administered, so adherence and thus effectiveness can be improved by reducing the duration of treatment. One option is to administer the same total dose over seven days instead of 14, but this high daily dose of primaquine increases the risk of gastrointestinal and haematological adverse events.

The safe and effective radical cure of $P$. vivax can be achieved with a variety of diagnostic and treatment strategies, which can be evaluated using cost-effectiveness analyses to determine the most efficient provision of healthcare. In order to do this, information needs to be gathered on the costs related to $P$. vivax and its treatment; to date, little research has been done in this important area [6] (Chapter 1). These challenges are highlighted in Chapter 4 which relied on cost data from disparate sources $[7,8,9,10,11,12,13]$, since many of the vivax-specific cost data available were from studies outside of Asia [14, 15, 16, 17].

A recent clinical trial comparing a seven day primaquine regimen with a 14 day regimen included costing surveys for households and health facilities within the study design [18], enabling rich insights into the household costs associated with $P$. vivax infection and the healthcare costs of providing the radical cure of malaria. The costs of vivax malaria, and different screening and treatment options, will inform the 
cost-effectiveness of different radical cure strategies to prevent relapses and onward transmission of the parasite.

The aim of this study is to describe the costs of uncomplicated $P$. vivax malaria infection, including the direct health expenditure for both healthcare providers and households, and the indirect cost to households due to lost productivity, and to compare these between different countries in the Middle East, Africa and Asia.

\subsection{Methods}

\subsubsection{Clinical trial details}

The Improving the radical cure of vivax malaria (IMPROV) study has been described previously [18]. In short, patients at sites in Afghanistan, Ethiopia, Indonesia and Vietnam were enrolled in a three-armed trial with the following comparators:

- Standard blood treatment plus 14 days of supervised primaquine (total dose 7.0 $\mathrm{mg} / \mathrm{kg})$

- Standard blood treatment plus 7 days of supervised primaquine (total dose 7.0 $\mathrm{mg} / \mathrm{kg}$ ) followed by 7 days of placebo

- Standard blood treatment plus 14 days placebo

Standard medical care and treatment for the blood-stage infections were in alignment with local malaria policies. Patients were screened for G6PD deficiency prior to enrollment. Individuals with G6PD deficiency were excluded from the randomised study; instead, they were offered enrolment in a single-arm observational study where they received standard treatment for blood-stage infections plus eight doses of weekly primaquine (total dose $6 \mathrm{mg} / \mathrm{kg}$ ). After $P$. vivax was diagnosed, patients were approached for informed consent. For any tests that were not routine for the study site, consent and assent were obtained from the patient before these tests were performed. Patients were followed up for 12 months following enrollment. The cost to the households and the healthcare providers were collected alongside the trial. 


\subsubsection{Household cost collection}

Household costs for individuals with $P$. vivax malaria were collected using standardised surveys translated into local languages; these reflect the cost of the patient's illness. Patients (or informants on the patients behalf) were interviewed by study staff at the initial visit (day 0) when recruited into the study and at a follow up visit 13 days after treatment for G6PD normal or 14 days after treatment if G6PD deficient. While the patients were followed up for 12 months, only the initial recruitment episode costs are reported to minimise any bias that could arise due to changes in treatment-seeking behaviour as a result of being enrolled in the study.

Direct costs reported in local currencies included the out of pocket expenditure for the entire episode from the patient's perspective. The location and cost of treatment seeking and transport to other healthcare facilities for the malaria episode were also included. Payments for treatment (medications, tests and fees) were included along with the cost of transportation to the healthcare facility, which was multiplied by two to account for the return journey. The costs of visiting the healthcare facility for the purpose of follow up visits for the clinical study were excluded.

Indirect costs included productivity losses due to illness. Patients reported the length of time that they could not perform usual activities due to illness and the length of time that the patient required a caregiver to stop doing usual work in order to care for them. The number of days reported at the initial and recurrent visits were summed and multiplied by the GDP per capita per day for 2016 to calculate the total indirect costs of illness [19].

\subsubsection{Provider cost data collection}

The costs of providing care from the healthcare provider perspective were taken from IMPROV study sites in Ethiopia (Arba Minch), Indonesia (North Sumatra) and Vietnam (Dak-O). Since the site in Ethiopia was a research facility, inputs were adjusted to reflect a routine clinical care setting. Micro-costing was used to collect costs for patient 
registration, physical exam, blood draw, malaria diagnosis, treatment, G6PD RDT, fluorescent spot test (FST) and the Haemocue ${ }^{\mathrm{TM}}$ test. Cost data were collected by local trial staff using a standardised tool.

The cost of usual care for P. vivax included the site-specific costs of diagnosis and treatment. Depending on the site, venous blood draw, finger prick blood draw, malaria RDT and microscopy were included in the usual care costs. One blood draw was taken for all tests undertaken for each patient. In Ethiopia usual care included the cost of a finger prick blood draw and microscopy. In Indonesia usual care included a finger prick blood draw and malaria RDT. In Vietnam, malaria RDT and microscopy are used together so the costs for both tests were included. Usual care for primaquine was also included: this was $0.25 \mathrm{mg} / \mathrm{kg}$ in Indonesia and Vietnam. In Ethiopia primaquine is not routinely prescribed.

Routine G6PD testing was not done at any of the sites, so the cost of RDT and FST were calculated as additional test costs. These tests needed to be repeated for some patients, so FST and G6PD RDT costs are presented as both per patient and per test. As throughputs were low, it was assumed that the FST would be used as a point-of-care test (i.e. only one test run in a batch). In addition, it was assumed that the storage of FST consumables would be in a freezer (or a fridge with a $-20^{\circ} \mathrm{C}$ freezer compartment) as this offered a more conservative estimate of costs.

Information on capital and recurrent costs were collected by direct observation and health staff interviews. Equipment costs were annuitised based on useful life estimates using the standard depreciation rate of $3 \%$ [20] and divided by the number of malaria patients or total patients who attend the facilities annually. Overhead costs were collected for each facility using the best available data and reported as monthly costs alongside the average number of patients the facility treated for all diseases. Personnel time were measured through time and motion studies. The time needed for both malaria and G6PD RDTs was assumed to be the same. The timing of FST testing was averaged from Indonesia and Vietnam. 


\subsubsection{Analysis}

All costs are reported in 2016 US $\$$. Costs collected in other years were inflated using GDP deflators [21] and then converted using the mid-year (1 July) exchange rates from 2016 [22]. For unit costs from 2017, a consumer price index (CPI) deflation factor of $2 \%$ was applied after converting to US\$ using the mid-year rate [23]. The midpoint of recruitment was used as the cost year for the data collection. To aid comparisons between countries in terms of the ability of each currency to buy the same amount of goods and services, the appendix contains the costs in international dollars (I\$) converted using Purchasing Power Parity (PPP) conversion factors [24].

The patient-level data were analysed using STATA 14.2 [25]. Patients who did not return at day 13 were excluded from the analysis. For those who did fill out surveys, missing data for costs were minimal $(<0.1 \%)$ and assumed to be zero. For missing data on duration of illness, five days was assumed for anyone who stated being ill for more than four days but did not specify the length of time.

The mean, SD, median and interquartile range are reported for the household costs. Mann-Whitney and Kruskal-Wallis tests were used for comparisons. For the provider costs, only point estimates were available so uncertainty cannot be ascertained. All components of the provider costs are reported as per patient costs unless otherwise noted. The incremental cost of switching from usual care to $0.5 \mathrm{mg} / \mathrm{kg}$ primaquine is reported as well as the incremental cost for switching from FST to G6PD RDT. Visit costs excluding the vivax-specific costs of drugs and diagnosis are included to aid comparisons with the standard visit costs reported by WHO [9].

\section{$5.3 \quad$ Results}

\subsubsection{Patient characteristics}

Results were available from a total of 2,008 patients, representing $93 \%(2,008 / 2,168)$ of those enrolled in the IMRPOV trial Figure 5.1. In total 37\% (745) of patients were 


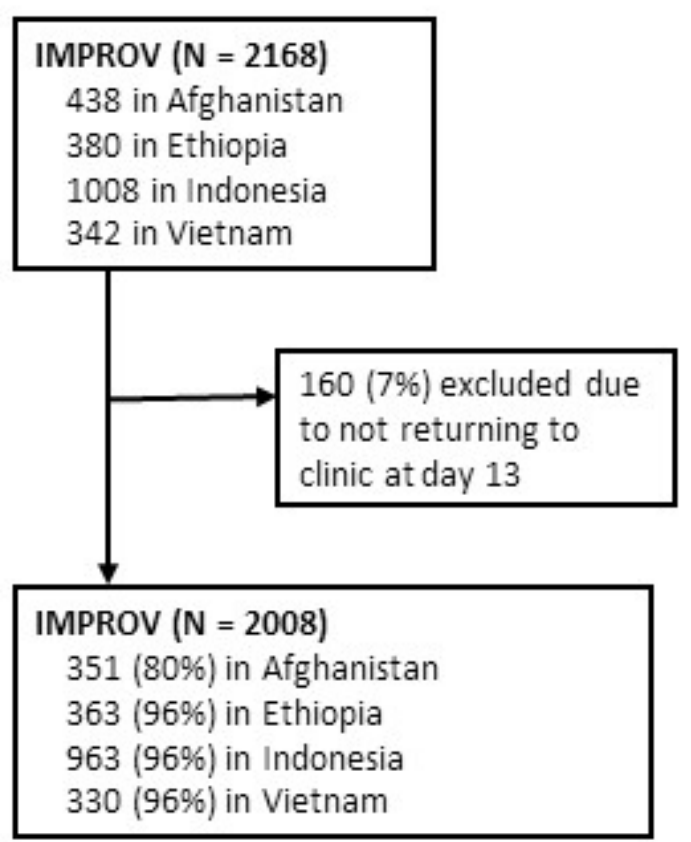

Figure 5.1: Flow diagram of patient recruitment to the trials and inclusion in the household cost study.

female. This bias was attributable mostly to the Vietnam site where 86\% (283/330) of patients were male. The age of the patients ranged from nine months to 94 years, with $42 \%$ of patients being children under 15 years old. The proportion of children under 15 varied by country with 14\% (45/330) in Vietnam, 37\% (134/363) in Ethiopia, 47\% (449/964) in Indonesia and 59\% (208/351) in Afghanistan. In the 2,007 patients who reported the duration of illness before attending the health centre, the mean number of days of illness was $2.5(\mathrm{SD}=2.3)$, ranging from 1.6 days $(\mathrm{SD}=1.3)$ in Vietnam to 3.3 days $(\mathrm{SD}=2.7)$ in Indonesia.

\subsubsection{Indirect household costs and usual activities}

At the initial visit, the number of days in which usual activities were not possible were consistently reported as being shorter than days of illness with a mean of 1.4 (SD = 1.6). This figure increased to 3.3 days $(\mathrm{SD}=3.0)$ for the entire episode (Table 5.1). For carers, the mean number of days of usual activity missed was $2.8(\mathrm{SD}=2.9)$. Patients in Indonesia reported the highest number of days missed from usual activities (mean = 3.9 ) and days required by a carer $($ mean $=2.7)$, compared to 2.3 and 1.2 in Afghanistan, 
Table 5.1: Days unable to do usual activities and days a caretaker is required by country and associated indirect costs (2016 US\$).

\begin{tabular}{|c|c|c|c|c|c|c|c|c|}
\hline \multirow[b]{2}{*}{ Country } & \multirow{2}{*}{$\begin{array}{l}\text { Patients } \\
\text { enrolled } \\
\mathrm{N}\end{array}$} & \multirow{2}{*}{$\begin{array}{l}\text { Patients } \\
\text { included } \\
\mathrm{n}(\%)\end{array}$} & \multicolumn{2}{|c|}{ Patient days lost } & \multicolumn{2}{|c|}{ Carer days lost } & \multirow{2}{*}{$\begin{array}{l}\text { Mean costs } \\
\text { for patients } \\
\text { (SD) }\end{array}$} & \multirow{2}{*}{$\begin{array}{l}\text { Mean costs } \\
\text { for carers } \\
\text { (SD) }\end{array}$} \\
\hline & & & $\begin{array}{l}\text { Mean } \\
(\mathrm{SD})\end{array}$ & $\begin{array}{l}\text { Median } \\
\text { (IQR) }\end{array}$ & $\begin{array}{l}\text { Mean } \\
(\mathrm{SD})\end{array}$ & $\begin{array}{l}\text { Median } \\
\text { (IQR) }\end{array}$ & & \\
\hline Afghanistan & 438 & $351(80 \%)$ & $2.3(1.3)$ & $2(1.5-3)$ & $1.2(1.2)$ & $1(0-2)$ & $3.4(2.0)$ & $1.8(1.8)$ \\
\hline Ethiopia & 380 & $363(96 \%)$ & $3.4(2.6)$ & $2.5(2-4)$ & $2.3(2.9)$ & $1.5(0-3)$ & $6.6(5.1)$ & $4.4(5.7)$ \\
\hline Indonesia & 1008 & $963(96 \%)$ & $3.9(3.7)$ & $3(0.5-5)$ & $2.7(3.6)$ & $1(0.5-4)$ & $37.8(36.6)$ & $26.8(35.5)$ \\
\hline Vietnam & 342 & $330(96 \%)$ & $2.8(1.9)$ & $2.5(1.5-3.5)$ & $0.9(1.2)$ & $0.5(0-1.5)$ & $16.5(11.1)$ & $5.5(7.1)$ \\
\hline Overall & 2,168 & $2,008(93 \%)$ & $3.3(3.0)$ & $2.5(1-4)$ & $2.8(3.0)$ & $1(0.5-3)$ & $22.7(29.9)$ & $14.9(27.4)$ \\
\hline
\end{tabular}

Table 5.2: Usual activities for patients and their carers - \% (n).

\begin{tabular}{llllll}
\hline Usual activity & Afghanistan & Ethiopia & Indonesia & Vietnam & Overall \\
\hline Patient - N & $\mathbf{3 5 1}$ & $\mathbf{3 6 3}$ & $\mathbf{9 6 4}$ & $\mathbf{3 3 0}$ & $\mathbf{2 , 0 0 8}$ \\
Housework (unpaid) & $14 \%(49)$ & $7 \%(27)$ & $13 \%(124)$ & $8 \%(28)$ & $11 \%(228)$ \\
Subsistence farming (unpaid) & $13 \%(47)$ & $1 \%(3)$ & $2 \%(21)$ & $77 \%(253)$ & $16 \%(324)$ \\
Attending school & $54 \%(189)$ & $53 \%(191)$ & $48 \%(467)$ & $9 \%(31)$ & $44 \%(878)$ \\
Paid employment & $3 \%(10)$ & $22 \%(81)$ & $4 \%(37)$ & $2 \%(5)$ & $7 \%(133)$ \\
Other & $16 \%(56)$ & $17 \%(61)$ & $33 \%(315)$ & $4 \%(13)$ & $22 \%(445)$ \\
Carer - N & $\mathbf{2 1 2}$ & $\mathbf{2 3 9}$ & $\mathbf{8 2 1}$ & $\mathbf{1 8 0}$ & $\mathbf{1 4 5 0}$ \\
Housework (unpaid) & $39 \%(83)$ & $30 \%(71)$ & $65 \%(535)$ & $33 \%(60)$ & $52 \%(749)$ \\
Subsistence farming (unpaid) & $23 \%(48)$ & $4 \%(10)$ & $4 \%(29)$ & $66 \%(118)$ & $14 \%(205)$ \\
Attending school & $9 \%(20)$ & $18 \%(43)$ & $3 \%(23)$ & $1 \%(1)$ & $6 \%(87)$ \\
Paid employment & $8 \%(18)$ & $32 \%(76)$ & $4 \%(36)$ & $1 \%(1)$ & $9 \%(131)$ \\
Other & $20 \%(43)$ & $16 \%(39)$ & $24 \%(198)$ & $0 \%(0)$ & $19 \%(280)$ \\
\hline
\end{tabular}

3.4 and 2.3 in Ethiopia, and 2.8 and 0.9 in Vietnam ( $p<0.001$ for both comparisons).

Overall, 44\% (878/2008) of patients reported that they would normally be attending school. In Vietnam, where patients were older, only $9 \%$ of individuals reported that they were missing school, but the majority $(77 \%, 253 / 330)$ reported missing their work as a subsistence farmer. Carers were required to cut back on usual activities for $72 \%$ $(1,450 / 2,008)$ of patients. While $52 \%$ of carers $(749 / 1,450)$ reported being unable to do housework, $14 \%$ (205) were unable to farm, 9\% (131) missed paid employment and 6\% (87) missed another activity. Table 5.2 details what usual activities entailed in each country.

\subsubsection{Treatment-seeking behaviour and associated costs}

In three of the four study countries the majority of patients reported having not sought treatment elsewhere: 91\% (319/351) in Afghanistan, 95\% (345/362) in Ethiopia and 

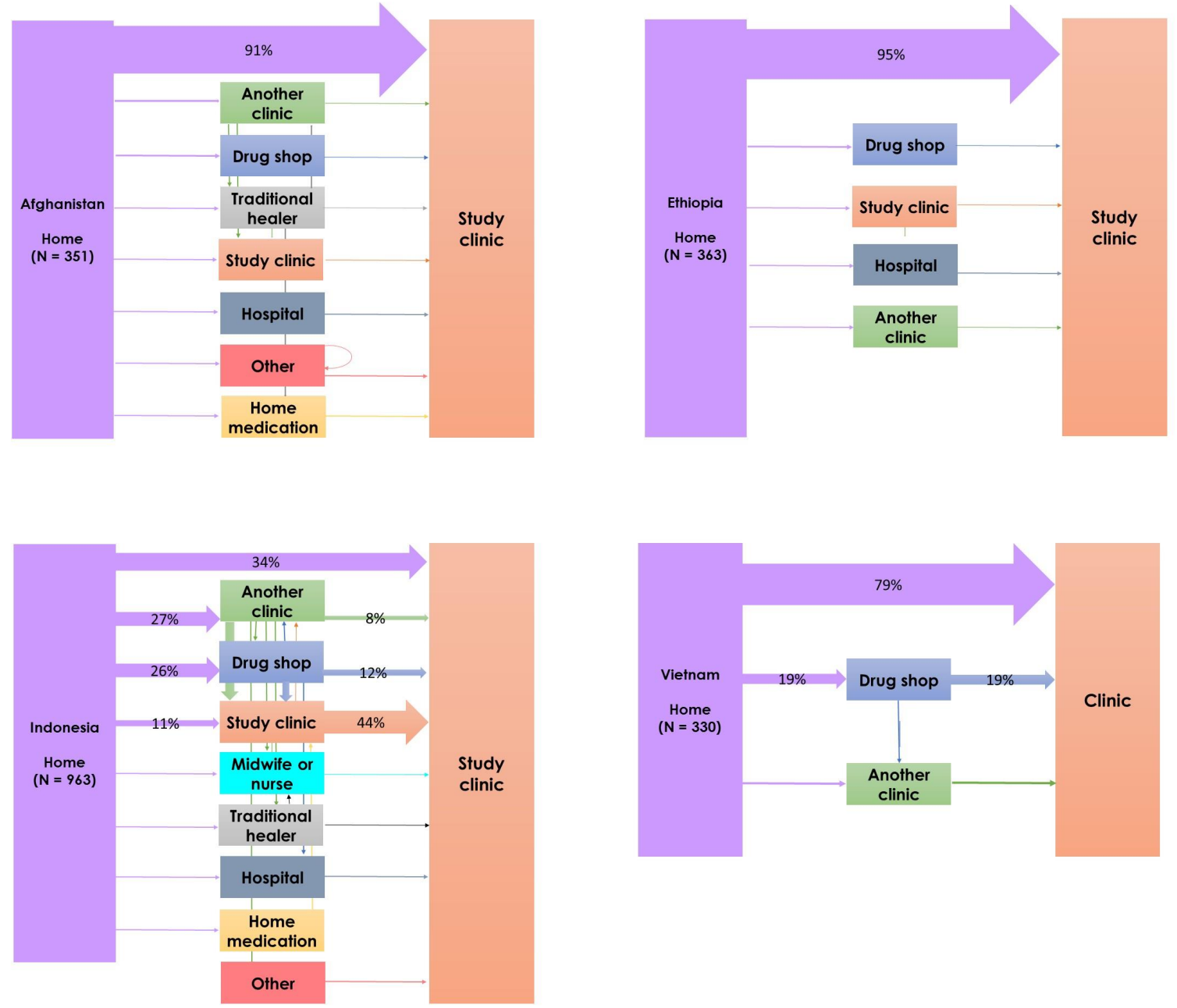

Figure 5.2: Patterns of treatment-seeking among trial participants prior to enrolment in the study. Only the first two treatment seeking destinations are shown here. Arrow sizes are in proportion to the percentage seeking treatment in that country with labels for patient flows involving more than $5 \%$ of the patient population.

$79 \%(260 / 330)$ in Vietnam (Figure 5.2). Of the 70 patients who sought treatment prior to enrolment in Vietnam, 64 (91\%) had visited a drug shop. In Indonesia only $34 \%(329 / 963)$ of patients were seeking treatment for the first time with 1\% (13/963) reporting that they sought treatment three times before study enrolment. The mean costs for treatment seeking at locations other than the study facility up until day 13 in Afghanistan, Ethiopia and Vietnam were less than a dollar as compared with Indonesia where the mean treatment and transport costs were US $\$ 1.4(\mathrm{SD}=6.4)$ and US $\$ 0.5$ $(\mathrm{SD}=0.9)$, respectively (Table 5.3$)$. 
Table 5.3: Direct, indirect and total household costs in 2016 US $\$$ per patient with vivax malaria $(\mathrm{N}=2007)$. Other treatment and other transport include all costs of treatment seeking at other locations up to day $13 .^{*}$

\begin{tabular}{|c|c|c|c|c|c|c|}
\hline \multirow{2}{*}{ Country } & \multicolumn{2}{|c|}{ Direct costs } & \multicolumn{2}{|c|}{ Indirect costs } & \multicolumn{2}{|c|}{ Total costs } \\
\hline & Mean (SD) & Median (IQR) & Mean (SD) & Median (IQR) & Mean (SD) & Median (IQR) \\
\hline Afghanistan total & $3.1(2.2)$ & $2.9(1.8-3.8)$ & $5.3(3.0)$ & $5.4(3.1-6.2)$ & $8.4(4.2)$ & $7.9(4.8-10.5)$ \\
\hline Other treatment & $0.3(1.6)$ & $0.0(0.0-0.0)$ & & & & \\
\hline Other transport & $0.1(0.4)$ & $0.0(0.0-0.0)$ & & & & \\
\hline Visit cost & $0.0(0.2)$ & $0.0(0.0-0.0)$ & & & & \\
\hline Transport cost & $2.8(1.5)$ & $2.6(1.8-3.8)$ & & & & \\
\hline Ethiopia total & $2.4(1.6)$ & $2.3(1.7-2.9)$ & $11.1(9.3)$ & $7.8(4.9-14.6)$ & $13.5(9.67)$ & $10.3(7.1-17.1)$ \\
\hline Other treatment & $0.1(1.0)$ & $0.0(0.0-0.0)$ & & & & \\
\hline Other transport & $0.0(0.2)$ & $0.0(0.0-0.0)$ & & & & \\
\hline Visit cost & $0.5(0.7)$ & $0.3(0.0-0.5)$ & & & & \\
\hline Transport cost & $1.8(0.9)$ & $1.8(0.9-2.3)$ & & & & \\
\hline Indonesia total & $4.0(7.3)$ & $2.7(1.5-4.9)$ & $64.6(64.5)$ & $48.9(14.7-97.8)$ & $68.6(66.8)$ & $51.4(18.2-99.4)$ \\
\hline Other treatment & $1.4(6.4)$ & $0.1(0.0-1.5)$ & & & & \\
\hline Other transport & $0.5(0.9)$ & $0.0(0.0-0.8)$ & & & & \\
\hline Visit cost & $0.5(1.0)$ & $0.0(0.0-1.1)$ & & & & \\
\hline Transport cost & $1.6(1.4)$ & $1.4(0.8-1.5)$ & & & & \\
\hline Vietnam total & $3.7(2.6)$ & $2.7(2.7-4.0)$ & $22.0(15.7)$ & $18.0(12.0-30.0)$ & $25.7(16.4)$ & $21.6(14.7-33.2)$ \\
\hline Other treatment & $0.8(2.1)$ & $0.0(0.0-0.0)$ & & & & \\
\hline Other transport & $0.2(0.6)$ & $0.0(0.0-0.0)$ & & & & \\
\hline Visit cost & $0.0(0.0)$ & $0.0(0.0-0.0)$ & & & & \\
\hline Transport cost & $2.7(1.0)$ & $2.7(1.8-3.6)$ & & & & \\
\hline Overall total & $3.5(5.3)$ & $2.7(1.8-4.1)$ & $37.5(52.7)$ & $14.7(6.2-48.9)$ & $41.1(54.2)$ & $18.6(10.0-51.5)$ \\
\hline
\end{tabular}

* See Appendix Table 5.9 for values converted using the PPP conversion factor.

\subsubsection{Direct and indirect costs to patients from the enrolment up to the follow up visit}

The mean total direct cost to patients ranged from US\$2.4 (SD = 1.6) in Ethiopia to US\$4.0 (SD = 7.3) in Indonesia (Table 5.3). The mean cost of transportation ranged from US\$1.6 $(\mathrm{SD}=1.4)$ in Indonesia to US\$2.8 $(\mathrm{SD}=1.5)$ in Afghanistan (Table 3). Overall, $93 \%(1,869 / 2,005)$ of patients reporting a journey of less than one hour. The majority of those reporting long travel times were in Afghanistan where 28\% (99/351), taking longer than one hour to get to the clinic. In Afghanistan differences in indirect costs were apparent by age and differences in direct and total costs were apparent by gender (Table 5.4). In Ethiopia and Indonesia, differences in indirect and total costs were apparent by age (Table 5.4). 
Table 5.4: Median costs (IQR) for each country compared by age and gender using the Mann-Whitney test (2016 US\$).*

\begin{tabular}{|c|c|c|c|c|c|c|}
\hline & \multicolumn{3}{|c|}{ Gender } & \multicolumn{3}{|c|}{ Age } \\
\hline & Male & Female & $\mathrm{p}$ value & $15+$ years & $<15$ years & $\mathrm{p}$ value \\
\hline Afghanistan - N (\%) & $239(68 \%)$ & $112(32 \%)$ & & $143(41 \%)$ & $208(59 \%)$ & \\
\hline Direct costs & $2.9(1.8-3.8)$ & $3.8(2.1-3.8)$ & 0.005 & $2.9(1.8-3.8)$ & $2.9(1.8-3.8)$ & 0.861 \\
\hline Indirect costs & $4.6(3.1-6.2)$ & $6.2(3.1-7.7)$ & 0.007 & $4.6(3.1-6.2)$ & $6.2(3.1-6.2)$ & 0.019 \\
\hline Total costs & $7.4(4.5-10.0)$ & $10.0(5.9-11.5)$ & 0.004 & $6.9(4.6-10.6)$ & $9.1(5.3-10.4)$ & 0.191 \\
\hline Ethiopia - N (\%) & $212(58 \%)$ & $151(42 \%)$ & & $229(63 \%)$ & $134(37 \%)$ & \\
\hline Direct costs & $2.1(1.7-2.9)$ & $2.3(1.8-3.0)$ & 0.615 & $2.3(1.8-2.9)$ & $2.2(1.7-3.0)$ & 0.981 \\
\hline Indirect costs & $7.8(3.9-13.6)$ & $9.7(5.8-15.5)$ & 0.056 & $7.8(3.9-13.6)$ & $10.2(5.8-16.5)$ & $<0.001$ \\
\hline Total costs & $9.7(6.9-16.1)$ & $12.0(7.5-17.8)$ & 0.093 & $9.6(6.6-15.2)$ & $12.6(8.7-18.9)$ & 0.001 \\
\hline Indonesia - N (\%) & $529(55 \%)$ & $435(45 \%)$ & & $515(53 \%)$ & $449(47 \%)$ & \\
\hline Direct costs & $2.7(1.5-5.0)$ & $2.7(1.5-4.8)$ & 0.750 & $2.8(1.5-5.2)$ & $2.6(1.5-4.6)$ & 0.256 \\
\hline Indirect costs & $44.0(9.8-97.8)$ & $58.7(14.7-97.8)$ & 0.064 & $34.2(9.8-78.2)$ & $58.7(19.6-97.8)$ & $<0.001$ \\
\hline Total costs & $51.4(18.2-99.2)$ & $59.4(21.1-100.6)$ & 0.096 & $40.0(14.4-82.2)$ & $61.4(24.4-104.3)$ & $<0.001$ \\
\hline Vietnam - N (\%) & $283(86 \%)$ & $47(14 \%)$ & & $285(86 \%)$ & $45(14 \%)$ & \\
\hline Direct costs & $2.7(1.8-3.6)$ & $3.6(2.7-5.4)$ & 0.073 & $2.7(2.2-3.6)$ & $2.7(2.7-4.5)$ & 0.222 \\
\hline Indirect costs & $18.0(12.0-27.0)$ & $24.0(12.0-35.9)$ & 0.104 & $18.0(12.0-30.0)$ & $18.0(12.0-30.0)$ & 0.600 \\
\hline Total costs & $21.1(14.4-32.6)$ & $25.8(16.8-43.1)$ & 0.065 & $21.6(14.4-33.5)$ & $23.2(17.7-32.6)$ & 0.352 \\
\hline
\end{tabular}

* See Appendix Table 5.10 for values converted using the PPP conversion factor.

\subsubsection{Provider costs}

For the healthcare provider, the total cost of usual care per patient treated ranged from US $\$ 8.9$ in Vietnam to $\$ 12.4$ in Indonesia (Table 5.5). The lower costs in Vietnam were due to the lower cost of goods alongside high clinic throughputs for all patients. Table 5.6 provides details on the overhead components. When excluding the costs of drugs and diagnostics (including all laboratory costs), the total visit cost was US $\$ 6.7$ in Ethiopia, US\$12.7 in Indonesia and US\$4.0 in Vietnam.

Table 5.5: Cost of usual care for P. vivax infections (2016 US\$).*

\begin{tabular}{|c|c|c|c|c|c|c|}
\hline Country & Staff & Consumables & Equipment & Overheads & Total & Notes \\
\hline Ethiopia & 0.8 & 6.0 & 0.5 & 5.0 & 12.3 & $\begin{array}{l}\text { Includes finger prick blood draw } \\
\text { and microscopy. }\end{array}$ \\
\hline Indonesia & 1.5 & 9.1 & 0.4 & 4.2 & 15.2 & $\begin{array}{l}\text { Includes finger prick blood draw, } \\
\text { malaria RDT and primaquine } \\
(0.25 \mathrm{mg} / \mathrm{kg}) \text {. }\end{array}$ \\
\hline Vietnam & 1.0 & 3.1 & 2.4 & 1.9 & 8.3 & $\begin{array}{l}\text { Includes venous blood draw, } \\
\text { malaria RDT, microscopy and } \\
\text { primaquine }(0.25 \mathrm{mg} / \mathrm{kg}) \text {. }\end{array}$ \\
\hline
\end{tabular}

* See Appendix Table 5.11 for values converted using the PPP conversion factor. 
Table 5.6: Breakdown of reported monthly patient attendance and overhead costs (2016 $\mathrm{US} \$)^{*}$

\begin{tabular}{lrrr}
\hline Overhead & Ethiopia & Indonesia & Vietnam \\
\hline Number of patients per month & 600 & 250 & 1200 \\
Costs & & & \\
$\quad$ Cleaning and laundry & 783.2 & 118.3 & 87.9 \\
$\quad$ Electricity & 13.4 & 59.1 & 109.9 \\
Fuel for transformer & - & 465.4 & - \\
Internet & 158.3 & 72.5 & 9.7 \\
Maintenance & 44.8 & - & 175.8 \\
Building cost or rent & 1941.5 & 158.9 & 1851.3 \\
Security & - & 167.8 & - \\
Telephone & 67.1 & - & 8.3 \\
Water & 17.9 & - & - \\
\hline Total & $\mathbf{5 9 9 4 . 2}$ & $\mathbf{1 0 4 2 . 1}$ & $\mathbf{2 2 4 2 . 8}$ \\
\hline
\end{tabular}

* See Appendix Table 5.12 for values converted using the PPP conversion factor. 
Table 5.7: Additional cost per patient and time per test required for laboratory tests (2016 US\$).*

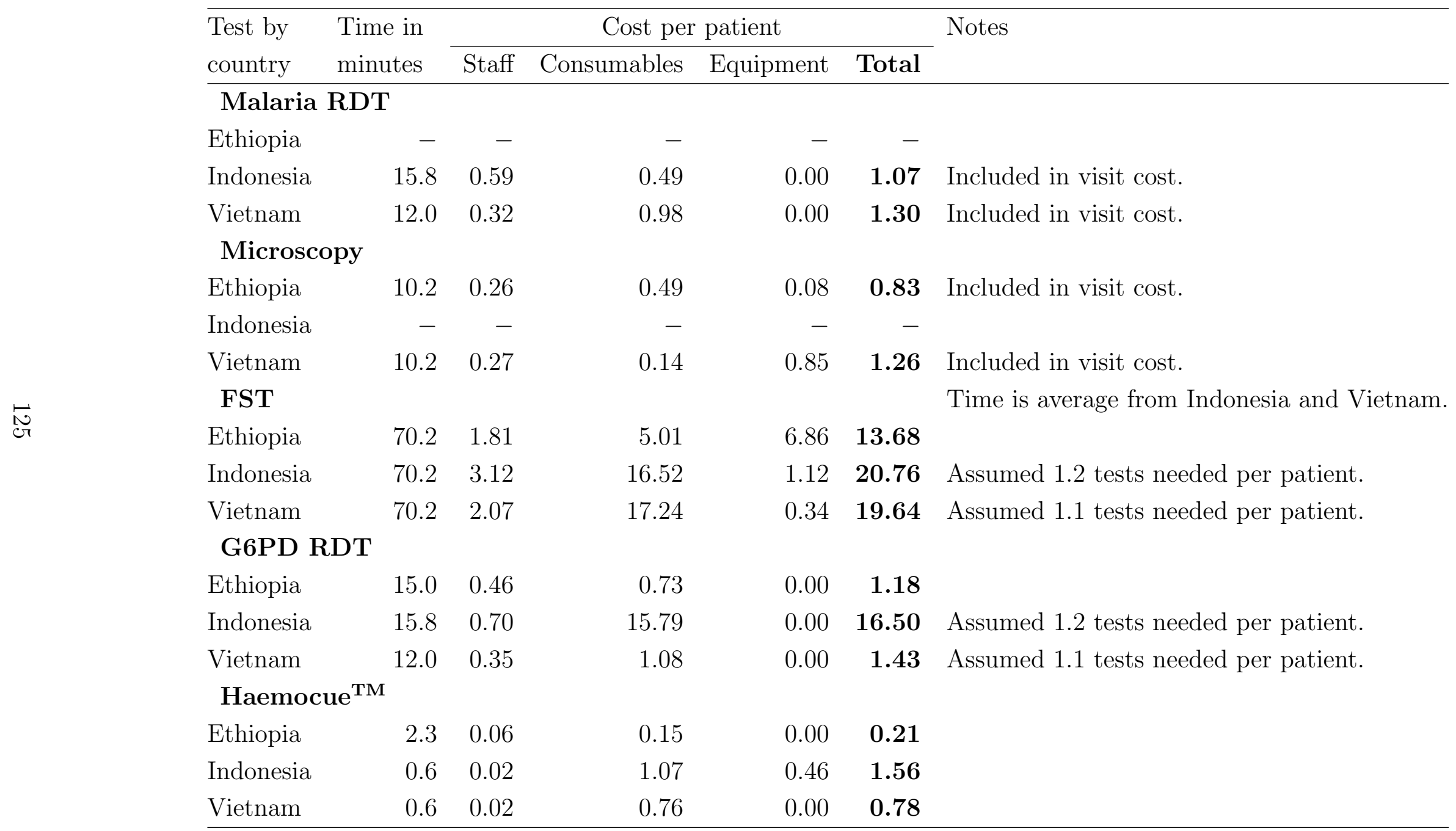

\footnotetext{
* See Appendix Table 5.14 for values converted using the PPP conversion factor and Table 5.8 for the per test costs.
} 
Table 5.8: Cost per FST and G6PD RDT administered (2016 US\$).*

\begin{tabular}{lrrrr}
\hline Test by country & Staff & Consumables & Equipment & Total \\
\hline FST & & & & \\
Ethiopia & 1.81 & 5.01 & 6.86 & $\mathbf{1 3 . 6 8}$ \\
Indonesia & 2.60 & 13.76 & 1.12 & $\mathbf{1 7 . 4 9}$ \\
Vietnam & 1.88 & 15.67 & 0.34 & $\mathbf{1 7 . 8 9}$ \\
$\quad$ G6PD RDT & & & & \\
Ethiopia & 0.35 & 0.56 & 0.00 & $\mathbf{0 . 9 1}$ \\
Indonesia & 0.70 & 13.16 & 0.00 & $\mathbf{1 3 . 8 6}$ \\
Vietnam & 0.32 & 0.98 & 0.00 & $\mathbf{1 . 3 0}$ \\
\hline
\end{tabular}

* See Appendix Table 5.13 for values converted using the PPP conversion factor.

Table 5.7 shows the cost per patient and the associated laboratory time required. Implementing FST in these settings with relatively low throughputs resulted in a cost per test ranging from US\$13.7-17.9 for FST compared to US\$0.9-13.9 for G6PD RDT (Table 5.8). Indonesia and Vietnam reported having to repeat both FST and G6PD RDT for 10-20\% of patients, increasing the cost of screening per patient (Table 5.7). G6PD RDT was consistently cheaper than FST per patient, even in Indonesia where the unit cost of a G6PD RDT was US\$13.2. The potential cost savings per patient treated using a G6PD RDT instead of FST were US\$6.9 in Indonesia and US\$18.2 in Vietnam. Other vivax-specific costs included primaquine (US\$0.43 to increase from $0.25 \mathrm{mg} / \mathrm{kg}$ to $0.5 \mathrm{mg} / \mathrm{kg}$ and US\$0.86 if not using primaquine) and the cost of a Haemocue ${ }^{\mathrm{TM}}$ test, which ranged from US\$0.78 in Vietnam to US\$1.56 in Indonesia.

\subsection{Discussion}

These cost data from four countries represent the largest collection on the cost of $P$. vivax malaria to both households and healthcare providers to date. Whilst the cost for facilities to provide standard care was similar across sites (US\$8.3-15.2), the mean total household cost per episode varied considerably (US\$8.4 in Afghanistan to US\$68.6 in Indonesia), reflecting differences in both duration of time off normal activities and GDP per capita. Treatment seeking at other locations prior to trial enrolment varied by country, ranging from $5 \%$ in Ethiopia to $66 \%$ in Indonesia. Since only the enrollment 
visit is included in this analysis, it is unlikely that treatment-seeking behaviour prior to visiting the clinic would be different. The direct costs of treatment seeking in Indonesia are similar to those reported by the hospital exit surveys in Timika (Chapter 2); however, the indirect costs are higher. The higher costs are likely due to the utilisation of a second interview to capture costs incurred after seeking treatment.

The total costs to the patient were consistently more than double that of the provider. The mean cost to the patient was US\$13.5 in Ethiopia, US\$25.7 in Vietnam, US\$68.6 in Indonesia compared to US\$6.7, US\$10.4 and US\$12.4 for the healthcare provider, respectively. As malaria tends to disproportionately impact the poor (Chapter 2), it could be that the use of GDP per capita per day for lost wages has resulted in an overestimate of the indirect costs of vivax malaria. For example, a recent study that used the monthly wages for agricultural workers reported a mean indirect cost of US\$3.8 per vivax episode in rural Ethiopia [26]. While Chapter 2 indicated that patients diagnosed with malaria at public providers were likely to experience similar costs regardless of whether they have $P$. vivax or $P$. falciparum, the aforementioned study from Ethiopia found a significant difference in indirect and total costs by species [26]. Furthermore, the healthcare facility costs reported here are consistently higher than the cost of a visit to an outpatient facility with beds reported in WHO-CHOICE (CHOosing Interventions that are Cost-Effective) [9] inflated to 2016 [21] and converted to US\$ [22] resulting in costs of US\$0.9 in Ethiopia, US\$2.7 for Vietnam and US\$5.3 in Indonesia. The costs presented here are 593\%, 7\% and 44\% higher, respectively. If patient costs were compared with the WHO-CHOICE visit costs plus malaria-specific costs instead of those found in this study, then the proportion of $P$. vivax costs borne by the household would be even higher.

While the focus of most costing studies is the direct costs of seeking treatment for malaria, such as payments for tests and transportation, previous research indicates that the indirect costs of lost productivity can be the largest cost component [27]. The results of this chapter are consistent with those findings: while the total costs varied by country, the largest cost component for households was consistently the indirect costs 
due to lost productivity. For many households, the burden of disease is not only the time that an individual cannot work or attend school due to illness or treatment seeking, but also the time required by another household member to care for that individual instead of performing his or her usual activities; in most places this constituted over $80 \%$ of the total cost of a malarial episode. The indirect costs were highest in Indonesia where individuals reported the most days off work for both patients and carers (3.9 and 2.7 days respectively). As $42 \%$ of the population were children, my methods for valuing the productivity costs may be an overestimate; however, this could be offset by educational losses [28, 29], which I did not attempt to include.

Previous studies reporting indirect costs in $P$. vivax patients have been limited. One vivax-specific study by Hansen and colleagues in Afghanistan reported patients with $P$. vivax personally losing almost a day and a half in addition to one half-day for their carers [16]. Other malaria studies that included vivax malaria patients showed approximately five productive days were lost for each malaria episode in Vietnam [30] and 14 days in Ethiopia [31]. While a better understanding of productivity losses is important for quantifying the burden of vivax malaria, their inclusion in economic evaluation can be controversial due to concerns around double counting (with productivity losses already potentially factored in to the valuation of disability or quality of life used to construct DALYs and QALYs, respectively). Many malaria studies use the outcome of infections averted rather than DALYs or focus on the years of life lost rather than the years of life with disability since the time with illness is often too small to have a significant impact on the ICER. These losses become more important for P. vivax malaria, which rarely results in direct mortality but often results in long-term morbidity [32, 33].

The ability to test for G6PD deficiency as advocated for ensuring safe radical cure by the WHO [3] resulted in additional costs ranging from US\$1.2 for the RDT in Ethiopia to US\$20.8 for the FST in Indonesia. At approximately US\$20.0 per test the FST is an expensive option for facilities with low numbers of $P$. vivax cases, more than doubling the cost of a visit for malaria. The main reasons for this incremental cost was the limited lifetime of the reagents. The G6PD RDT was consistently less expensive than 
the FST, but the costs savings were dependent on in-country costs, ranging from US $\$ 1.4$ per person in Vietnam to US\$13.9 in Indonesia. In settings such as those found in Indonesia, where primaquine is prescribed without G6PD testing, it will be difficult to convince policymakers to implement G6PD testing since the incremental cost per person treated is so high. Finally, if primaquine treatment is to be routinely administered, the additional use of a Haemocue ${ }^{\mathrm{TM}}$ test (or equivalent) might be viewed as a necessity that needs to be factored into the cost estimates. While it is a relatively inexpensive test (less than US\$2.0), it is likely that facilities would want to use it more than once per P. vivax episode to monitor patients for anaemia.

This study has a number of limitations. While uncertainty intervals were reported where possible, only point estimates were available for the provider costs (Tables 5.5 to 5.8). As these were collected remotely, these are likely to have a great deal of uncertainty, which could not be quantified in a meaningful way. While every effort was made to ensure that these were collected systematically, it is likely that some bias was introduced through the use of local staff to collect the costs. Accordingly, the provider cost data are not as reliable as the patient cost data. The provider costs are for those patients diagnosed with malaria and does not include the costs of diagnosing malaria in those who test negative. Since the cost of diagnostic tests are included, these could be used in cost-effectiveness analyses alongside the diagnostic accuracy to determine the overall screening costs. Since much of the data were gathered in the setting of a clinical trial, it is possible that direct payments for treatment may have been lower than normal. Finally, some settings may prefer to use a fridge for the storage of FST reagents rather than purchasing a freezer. While that would be less expensive in the short-term, the overall cost per test would increase as the reagents last twice as long in the freezer unless the fridge has a $-20^{\circ} \mathrm{C}$ compartment as the facilities in Ethiopia and Vietnam did. The cost of FST would decrease for facilities that treat a higher number of vivax patients.

In conclusion, the burden of $P$. vivax malaria is substantial at the household level with the largest component being productivity losses. Ensuring safe radical cure through 
G6PD RDTs should enable a reduction in this burden, but high test costs may impede their uptake. The provider costs presented from three countries and patient costs from four countries represent the largest collection of costs resulting from vivax malaria alone, and an important resource with which to embark upon more robust cost-effectiveness estimates in the future. 


\subsection{Appendix: Tables in international dollars}

The following tables present costs in $2016 \mathrm{I} \$$ converted using the PPP conversion factor.

These correspond to Tables 5.3-5.8.

Table 5.9: Direct, indirect and total household costs in 2016 I $\$$ per patient with vivax malaria $(\mathrm{N}=2007)$. Other treatment and other transport include all costs of treatment seeking at other locations up to day 13. (From Table 5.3.)

\begin{tabular}{|c|c|c|c|c|c|c|}
\hline \multirow{2}{*}{ Country } & \multicolumn{2}{|c|}{ Direct costs } & \multicolumn{2}{|c|}{ Indirect costs } & \multicolumn{2}{|c|}{ Total costs } \\
\hline & Mean (SD) & Median (IQR) & Mean (SD) & Median (IQR) & Mean (SD) & Median (IQR) \\
\hline Afghanistan total & $11.1(7.9)$ & $10.2(6.1-13.9)$ & $5.3(3.0)$ & $5.4(3.1-6.2)$ & $16.4(9.1)$ & $14.8(9.7-20.1)$ \\
\hline Other treatment & $0.9(5.5)$ & $0.0(0.0-0.0)$ & & & & \\
\hline Other transport & $0.3(1.3)$ & $0.0(0.0-0.0)$ & & & & \\
\hline Visit cost & $0.1(0.6)$ & $0.0(0.0-0.0)$ & & & & \\
\hline Transport cost & $9.8(5.5)$ & $9.2(6.1-13.9)$ & & & & \\
\hline Ethiopia total & $6.1(4.0)$ & $5.9(4.4-7.4)$ & $11.1(9.3)$ & $7.8(4.9-14.6)$ & $17.2(10.8)$ & $14.8(10.1-21.4)$ \\
\hline Other treatment & $0.2(2.5)$ & $0.0(0.0-0.0)$ & & & & \\
\hline Other transport & $0.1(0.4)$ & $0.0(0.0-0.0)$ & & & & \\
\hline Visit cost & $1.1(1.7)$ & $0.7(0.0-1.3)$ & & & & \\
\hline Transport cost & $4.7(2.4)$ & $4.7(2.3-5.8)$ & & & & \\
\hline Indonesia total & $12.9(23.3)$ & $8.6(4.9-15.6)$ & $64.6(64.5)$ & $48.9(14.7-97.8)$ & $77.5(73.4)$ & $60.8(27.6-105.1)$ \\
\hline Other treatment & $4.5(20.8)$ & $0.2(0.0-4.9)$ & & & & \\
\hline Other transport & $1.7(3.0)$ & $0.0(0.0-0.0)$ & & & & \\
\hline Visit cost & $1.6(3.2)$ & $0.0(0.0-3.7)$ & & & & \\
\hline Transport cost & $5.1(4.5)$ & $4.4(2.4-4.9)$ & & & & \\
\hline Vietnam total & $10.9(7.6)$ & $7.9(7.9-11.9)$ & $22.0(15.7)$ & $18.0(12.0-30.0)$ & $32.9(18.7)$ & $28.5(19.9-41.2)$ \\
\hline Other treatment & $2.1(6.1)$ & $0.0(0.0-0.0)$ & & & & \\
\hline Other transport & $0.7(1.7)$ & $0.0(0.0-2.4)$ & & & & \\
\hline Visit cost & $0.0(0.0)$ & $0.0(0.0-0.0)$ & & & & \\
\hline Transport cost & $8.0(2.9)$ & $7.9(5.3-10.6)$ & & & & \\
\hline Overall total & $11.0(17.0)$ & $8.1(5.3-13.9)$ & $37.5(52.7)$ & $14.7(6.2-48.9)$ & $48.6(59.0)$ & $25.9(14.4-61.7)$ \\
\hline
\end{tabular}


Table 5.10: Median costs (IQR) for each country compared by age and gender using the Mann-Whitney test (2016 I\$) from Table 5.4.

\begin{tabular}{clllllr}
\hline & \multicolumn{3}{c}{ Gender } & & \multicolumn{2}{c}{ Age } \\
\cline { 2 - 6 } & Male & Female & p value & $15+$ years & $<15$ years & p value \\
\hline Afghanistan - N (\%) & $239(68 \%)$ & $112(32 \%)$ & & $143(41 \%)$ & $208(59 \%)$ & \\
Direct costs & $10.2(6.1-13.9)$ & $13.9(7.4-13.9)$ & $\mathbf{0 . 0 0 5}$ & $10.2(6.1-13.9)$ & $10.2(6.1-13.9)$ & 0.844 \\
Indirect costs & $4.6(3.1-6.2)$ & $6.2(3.1-7.7)$ & $\mathbf{0 . 0 0 7}$ & $4.6(3.1-6.2)$ & $6.2(3.1-6.2)$ & $\mathbf{0 . 0 1 9}$ \\
Total costs & $13.3(9.7-20.1)$ & $19.7(11.2-21.6)$ & $\mathbf{0 . 0 0 2}$ & $14.3(9.7-20.3)$ & $15.9(10.2-20.1)$ & 0.407 \\
Ethiopia - N (\%) & $212(58 \%)$ & $151(42 \%)$ & & $229(63 \%)$ & $134(37 \%)$ & \\
Direct costs & $5.5(4.4-7.4)$ & $5.9(4.5-7.6)$ & 0.616 & $5.9(4.5-7.3)$ & $5.6(4.4-7.7)$ & 0.981 \\
Indirect costs & $7.8(3.9-13.6)$ & $9.7(5.8-15.5)$ & 0.056 & $7.8(3.9-13.6)$ & $10.2(5.8-16.5)$ & $<\mathbf{0 . 0 0 1}$ \\
Total costs & $13.7(10.1-20.9)$ & $15.3(10.1-22.0)$ & 0.199 & $13.6(9.3-20.0)$ & $15.8(11.2-22.9)$ & $\mathbf{0 . 0 0 6}$ \\
Indonesia - N $(\%)$ & $529(55 \%)$ & $435(45 \%)$ & & $515(53 \%)$ & $449(47 \%)$ & \\
Direct costs & $8.7(4.9-15.9)$ & $8.6(1.5-4.8)$ & 0.754 & $9.0(4.9-16.6)$ & $8.3(4.9-14.7)$ & 0.256 \\
Indirect costs & $44.0(9.8-97.8)$ & $58.7(4.9-15.9)$ & 0.064 & $34.2(9.8-78.2)$ & $58.7(19.6-97.8)$ & $<\mathbf{0 . 0 0 1}$ \\
Total costs & $55.5(25.7-102.9)$ & $62.6(30.1-111.2)$ & 0.136 & $47.6(23.5-92.9)$ & $68.9(35.4-112.9)$ & $<\mathbf{0 . 0 0 1}$ \\
Vietnam - N $(\%)$ & $283(86 \%)$ & $47(14 \%)$ & & $285(86 \%)$ & $45(14 \%)$ & \\
Direct costs & $7.9(5.3-10.6)$ & $10.6(7.9-15.9)$ & 0.073 & $7.9(6.6-10.6)$ & $7.9(7.9-13.2)$ & 0.226 \\
Indirect costs & $18.0(12.0-27.0)$ & $24.0(12.0-35.9)$ & 0.104 & $18.0(12.0-30.0)$ & $18.0(12.0-30.0)$ & 0.600 \\
Total costs & $28.5(19.9-40.5)$ & $31.9(22.9-52.9)$ & 0.066 & $23.2(19.9-41.2)$ & $31.2(23.3-43.6)$ & 0.202 \\
\hline
\end{tabular}

Table 5.11: Cost of usual care for P. vivax infections (2016 I\$) from Table 5.5.

\begin{tabular}{|c|c|c|c|c|c|c|}
\hline Country & Staff & Consumables & Equipment & Overheads & Total & Notes \\
\hline Ethiopia & 2.0 & 13.2 & 1.2 & 12.8 & 29.3 & $\begin{array}{l}\text { Includes finger prick blood draw } \\
\text { and microscopy. }\end{array}$ \\
\hline Indonesia & 4.9 & 15.0 & 1.4 & 13.3 & 34.6 & $\begin{array}{l}\text { Includes finger prick blood draw, } \\
\text { malaria RDT and primaquine } \\
(0.25 \mathrm{mg} / \mathrm{kg}) \text {. }\end{array}$ \\
\hline Vietnam & 3.0 & 5.7 & 6.9 & 5.5 & 21.1 & $\begin{array}{l}\text { Includes venous blood draw, } \\
\text { malaria RDT, microscopy and } \\
\text { primaquine }(0.25 \mathrm{mg} / \mathrm{kg}) \text {. }\end{array}$ \\
\hline
\end{tabular}


Table 5.12: Breakdown of reported monthly patient attendance and overhead costs (2016 I\$) from Table 5.6.

\begin{tabular}{lrrr}
\hline Overhead & Ethiopia & Indonesia & Vietnam \\
\hline Number of patients per month & 600 & 250 & 1200 \\
Costs & & & \\
$\quad$ Cleaning and laundry & 1993.5 & 378.7 & 259.2 \\
$\quad$ Electricity & 34.2 & 189.4 & 323.9 \\
Fuel for transformer & - & 1490.4 & - \\
$\quad$ Internet & 402.8 & 232.1 & 28.5 \\
Maintenance & 113.9 & - & 518.3 \\
$\quad$ Building cost or rent & 4941.9 & 509.0 & 5459.0 \\
$\quad$ Security & - & 537.5 & - \\
Telephone & 170.9 & - & 24.6 \\
$\quad$ Water & 45.6 & - & - \\
\hline Total & $\mathbf{7 7 0 2 . 7}$ & $\mathbf{3 3 3 7 . 1}$ & $\mathbf{6 6 1 3 . 5}$ \\
\hline
\end{tabular}

Table 5.13: Cost per FST and G6PD RDT administered (2016 I\$) from Table 5.8.

\begin{tabular}{lrrrr}
\hline Test by country & Staff & Consumables & Equipment & Total \\
\hline FST & & & & \\
Ethiopia & 4.62 & 9.27 & 13.99 & 27.88 \\
Indonesia & 8.34 & 39.36 & 3.50 & 51.20 \\
Vietnam & 5.55 & 39.44 & 0.99 & 45.97 \\
G6PD RDT & & & & \\
Ethiopia & 0.90 & 1.59 & 0.00 & 2.49 \\
Indonesia & 2.25 & 42.15 & 0.00 & 44.39 \\
Vietnam & 0.95 & 0.98 & 0.00 & 1.93 \\
\hline
\end{tabular}


Table 5.14: Additional cost per patient and time per test required for laboratory tests (2016 I\$) from Table 5.7.

\begin{tabular}{|c|c|c|c|c|c|c|}
\hline \multirow{2}{*}{$\begin{array}{l}\text { Test by } \\
\text { country }\end{array}$} & \multirow{2}{*}{$\begin{array}{l}\text { Time in } \\
\text { minutes }\end{array}$} & \multicolumn{4}{|c|}{ Cost per patient } & \multirow[t]{2}{*}{ Notes } \\
\hline & & Staff & Consumables & Equipment & Total & \\
\hline \multicolumn{7}{|c|}{ Malaria RDT } \\
\hline Ethiopia & - & - & - & - & - & \\
\hline Indonesia & 15.8 & 1.87 & 0.49 & 0.00 & 2.36 & Included in visit cost. \\
\hline Vietnam & 12.0 & 0.95 & 0.98 & 0.00 & 1.93 & Included in visit cost. \\
\hline \multicolumn{7}{|c|}{ Microscopy } \\
\hline Ethiopia & 10.2 & 0.67 & 1.23 & 0.21 & 2.11 & Included in visit cost. \\
\hline Indonesia & - & - & - & - & - & \\
\hline Vietnam & 10.2 & 0.81 & 0.25 & 2.50 & 3.56 & Included in visit cost. \\
\hline FST & & & & & & Time is average from Indonesia and Vietnam. \\
\hline Ethiopia & 70.2 & 4.62 & 9.27 & 13.99 & 27.88 & \\
\hline Indonesia & 70.2 & 10.01 & 47.24 & 3.50 & 60.74 & Assumed 1.2 tests needed per patient. \\
\hline Vietnam & 70.2 & 6.10 & 43.38 & 0.99 & 50.47 & Assumed 1.1 tests needed per patient. \\
\hline \multicolumn{7}{|c|}{ G6PD RDT } \\
\hline Ethiopia & 15.0 & 1.17 & 2.07 & 0.00 & 3.23 & \\
\hline Indonesia & 15.8 & 2.25 & 42.15 & 0.00 & 44.39 & Assumed 1.2 tests needed per patient. \\
\hline Vietnam & 12.0 & 1.04 & 1.08 & 0.00 & 2.12 & Assumed 1.1 tests needed per patient. \\
\hline \multicolumn{7}{|c|}{ Haemocue $^{\mathrm{TM}}$} \\
\hline Ethiopia & 2.3 & 0.15 & 0.44 & 0.00 & 0.59 & \\
\hline Indonesia & 0.6 & 0.07 & 3.44 & 1.47 & 4.98 & \\
\hline Vietnam & 0.6 & 0.05 & 2.25 & 0.00 & 2.30 & \\
\hline
\end{tabular}




\subsection{Declarations}

\subsubsection{Acknowledgments}

I am grateful to Ayodhia P. Pasaribu, Pham Nguyen Huong Thu, Nguyen Thanh Thuy Nhien, Ngo Viet Thanh, Tran Tinh Hien, Tedla Teferi and Asrat Hailu for assistance with collecting the healthcare provider cost data.

\subsubsection{Ethics approval}

Ethical approval for the original protocol and included amendments (Version 5) is obtained from the following review boards: Oxford Tropical Research Ethics Committee OxTREC (Ref number 101413) and the Human Research Ethics Committee of the Northern Territory Department of Health, Australia HREC (Ref Number 131991). In addition local approvals were obtained from the National Bioethics Committee (NBC) in Pakistan (this site was dropped before start of enrolment), the Institutional Review Board, Ministry of Public Health, Afghanistan, the Health Research Ethics Committee, Faculty of Medicine University of Indonesia, Cipto Mangunkusumo Hospital, Jakarta, Indonesia, the Ministry of Health Evaluation Committee on Ethics in Biomedical Research Vietnam, the Institutional Scientific \& Ethical Review Committee of the Ethiopian Public Health Institute, the National Research Ethics Review Committee, Ethiopia and the Institutional Review Board of the Columbia University Medical Centre, US. 


\section{References for Chapter 5}

[1] Battle KE, Weiss DJ, Lucas T, Nguyen M, Bhatt S, Cameron E, et al. The global burden of Plasmodium vivax malaria: 2000-2016. (In preparation);

[2] World Health Organization. Control and elimination of plasmodium vivax malaria: a technical brief; 2015 .

[3] World Health Organisation. Guidelines for the Treatment of Malaria. 2015;

[4] Bruxvoort K, Goodman C, Kachur SP, Schellenberg D. How patients take malaria treatment: a systematic review of the literature on adherence to antimalarial drugs. PLoS One. 2014;9(1):e84555. doi:10.1371/journal.pone.0084555.

[5] Douglas NM, Poespoprodjo JR, Patriani D, Malloy MJ, Kenangalem E, Sugiarto $\mathrm{P}$, et al. Unsupervised primaquine for the treatment of Plasmodium vivax malaria relapses in southern Papua: A hospital-based cohort study. PLoS Med. 2017;14(8):e1002379. doi:10.1371/journal.pmed.1002379.

[6] White MT, Yeung S, Patouillard E, Cibulskis R. Costs and Cost-Effectiveness of Plasmodium vivax Control. Am J Trop Med Hyg. 2016;95(6 Suppl):52-61. doi:10.4269/ajtmh.16-0182.

[7] Mulligan JA, Fox-Rushby JA, Adam T, Johns B, Mills A. Unit Costs of Health Care Inputs in Low and Middle Income Regions; 2005.

[8] Lubell Y, Reyburn H, Mbakilwa H, Mwangi R, Chonya K, Whitty CJ, et al. The cost-effectiveness of parasitologic diagnosis for malaria-suspected patients in an era of combination therapy. Am J Trop Med Hyg. 2007;77(6 Suppl):128-32.

[9] World Health Organisation. WHO-CHOICE unit cost estimates for service delivery. 2011;Available from: http://www. who.int/choice/ cost-effectiveness/inputs/health_service/en/(Accessed: 02 February 2016). 
[10] Kyaw SS, Drake T, Ruangveerayuth R, Chierakul W, White NJ, Newton PN, et al. Cost of treating inpatient falciparum malaria on the Thai-Myanmar border. Malar J. 2014;13:416. doi:10.1186/1475-2875-13-416.

[11] Ley B, Luter N, Espino FE, Devine A, Kalnoky M, Lubell Y, et al. The challenges of introducing routine G6PD testing into radical cure: a workshop report. Malaria Journal. 2015;14(1):377.

[12] MSH (Management Sciences for Health). International Drug Price Indicator Guide, 2014 Edition. 2015;

[13] Kyaw SS, Drake T, Thi A, Kyaw MP, Hlaing T, Smithuis FM, et al. Malaria community health workers in Myanmar: a cost analysis. Malar J. 2016;15(1):41. doi:10.1186/s12936-016-1102-3.

[14] Lemma H, San Sebastian M, Lofgren C, Barnabas G. Cost-effectiveness of three malaria treatment strategies in rural Tigray, Ethiopia where both Plasmodium falciparum and Plasmodium vivax co-dominate. Cost Eff Resour Alloc. 2011;9:2. doi:10.1186/1478-7547-9-2.

[15] de Oliveira MR, Giozza SP, Peixoto HM, Romero GA. Cost-effectiveness of diagnostic for malaria in Extra-Amazon Region, Brazil. Malar J. 2012;11:390. doi:10.1186/1475-2875-11-390.

[16] Hansen KS, Grieve E, Mikhail A, Mayan I, Mohammed N, Anwar M, et al. Cost-effectiveness of malaria diagnosis using rapid diagnostic tests compared to microscopy or clinical symptoms alone in Afghanistan. Malar J. 2015;14:217. doi:10.1186/s12936-015-0696-1.

[17] Peixoto HM, Brito MA, Romero GA, Monteiro WM, de Lacerda MV, de Oliveira MR. Cost-effectiveness analysis of rapid diagnostic tests for G6PD deficiency in patients with Plasmodium vivax malaria in the Brazilian Amazon. Malar J. 2016;15(1):82. doi:10.1186/s12936-016-1140-x. 
[18] IMPROV Study Group. Improving the radical cure of vivax malaria (IMPROV): a study protocol for a multicentre randomised, placebo-controlled comparison of short and long course primaquine regimens. BMC Infect Dis. 2015;15:558. doi:10.1186/s12879-015-1276-2.

[19] The World Bank. GDP per capita (current US\$). 2016;Available from: http://data. worldbank.org/indicator/NY.GDP.PCAP.CD(Accessed: 23 August 2017).

[20] World Health Organization. WHO Guide to cost-effectiveness analysis. World Health Organisation; 2003.

[21] The World Bank. Inflation, GDP deflator (annual \%). 2016;Available from: wWw . data. worldbank .org/indicator/NY . GDP.DEFL. KD. ZG(Accessed: 08 September 2017).

[22] XE. Current and Historical Rate Tables. 2016;Available from: http: //www . xe . com/currencytables/?from=USD\&date=2016-07-01 (Accessed: 8 September 2017).

[23] United States Department of Labor. CPI Inflation Calculator. Accessed: 8 September 2017;Available from: https://data.bls.gov/cgi-bin/cpicalc.pl? cost $1=1.00 \&$ year $1=201707 \&$ year $2=201607$.

[24] The World Bank. PPP conversion factor, GDP (LCU per international \$). 2016; Available from: https://data.worldbank.org/indicator/PA.NUS.PPP(Accessed: 02 April 2018).

[25] StataCorp. Stata Statistical Software: Release 14. 2015;.

[26] Hailu A, Lindtjorn B, Deressa W, Gari T, Loha E, Robberstad B. Economic burden of malaria and predictors of cost variability to rural households in south-central Ethiopia. PLoS One. 2017;12(10):e0185315. doi:10.1371/journal.pone.0185315. 
[27] Wiseman V, Kim M, Mutabingwa TK, Whitty CJ. Cost-effectiveness study of three antimalarial drug combinations in Tanzania. PLoS Med. 2006;3(10):e373. doi:10.1371/journal.pmed.0030373.

[28] Vitor-Silva S, Reyes-Lecca RC, Pinheiro TR, Lacerda MV. Malaria is associated with poor school performance in an endemic area of the Brazilian Amazon. Malar J. 2009;8:230. doi:10.1186/1475-2875-8-230.

[29] Nankabirwa J, Wandera B, Kiwanuka N, Staedke SG, Kamya MR, Brooker SJ. Asymptomatic Plasmodium infection and cognition among primary schoolchildren in a high malaria transmission setting in Uganda. Am J Trop Med Hyg. 2013;88(6):1102-8. doi:10.4269/ajtmh.12-0633.

[30] Morel CM, Thang ND, Xa NX, Hung le X, Thuan le K, Van Ky P, et al. The economic burden of malaria on the household in south-central Vietnam. Malar J. 2008;7:166. doi:10.1186/1475-2875-7-166.

[31] Deressa W, Hailemariam D, Ali A. Economic costs of epidemic malaria to households in rural Ethiopia. Trop Med Int Health. 2007;12(10):1148-56. doi:10.1111/j.1365-3156.2007.01901.x.

[32] Douglas NM, Anstey NM, Buffet PA, Poespoprodjo JR, Yeo TW, White NJ, et al. The anaemia of Plasmodium vivax malaria. Malar J. 2012;11(1):135. doi:1475-2875-11-135 [pii] 10.1186/1475-2875-11-135.

[33] Douglas NM, Lampah DA, Kenangalem E, Simpson JA, Poespoprodjo JR, Sugiarto P, et al. Major burden of severe anemia from non-falciparum malaria species in Southern Papua: a hospital-based surveillance study. PLoS Med. 2013;10(12):e1001575; discussion e1001575. doi:10.1371/journal.pmed.1001575. 


\section{Chapter 6}

\section{Global economic costs due to vivax}

malaria treatment and the potential cost-benefit of radical cure

\subsection{Introduction}

Although $P$. vivax was once regarded as a benign infection, it is increasingly recognised that its ability to relapse results in a cumulative risk of anaemia and associated direct and indirect attributable mortality $[1,2]$. Women and young children are particularly at risk since $P$. vivax in pregnancy can cause premature delivery and low birth weight, which both contribute to perinatal and infant mortality $[2,3,4]$. Access to early diagnosis and highly effective antimalarial treatment, and intensive vector control measures have reduced the global burden of P. falciparum malaria by $40 \%$ since 2000 [5]; however, in areas where such control interventions have driven down falciparum malaria, there has been a relative rise in vivax malaria $[6,7]$.

P. vivax is more difficult to cure than P. falciparum, as the parasite is able to form dormant liver stages (hypnozoites) that reactivate periodically, causing recurrent infections (relapses) and further ongoing transmission [8], Relapses require a hypno- 
zonticidal drug for radical cure in addition to the schizonticidal drug for the blood stage infection. Primaquine is the only available drug for radical cure at present, but it causes haemolysis in those with G6PD deficiency. This haemolysis can range from mild to life-threatening, depending on the type of G6PD deficiency. Many countries do not use primaquine regularly due to concerns about triggering haemolysis. Recently, point-of-care RDTs for G6PD deficiency have become available. These tests have the potential to enable remote settings with limited laboratory equipment to test before prescribing primaquine, excluding those with G6PD deficiency. In addition these tests are less expensive than the fluorescent spot test (FST) (Chapter 5). Accordingly, widespread radical cure following G6PD screening represents a new opportunity for the control of vivax malaria.

The economic impact of malaria is huge, both to the individual and society. Previous work by Gallup and Sachs indicated that countries with a high percentage of the population at risk of $P$. falciparum infection were likely to experience reductions in growth as great as 2\% GDP per capita [9]. However Gallup and Sachs did not elucidate other economic impacts that are more challenging to ascertain, such as the demographic consequences of high childhood mortality rates, the ability to develop human and physical capital, decreases in tourism and foreign investment, and co-infections with other diseases [10]. These estimates were derived more than 15 years ago and focused on $P$. falciparum in Africa. The global economic impact of $P$. vivax has not been ascertained.

The economic impact of vivax malaria is more challenging to estimate for a number of reasons. Whilst the risk of acute mortality is considerably lower following $P$. vivax infections compared to $P$. falciparum, when patients with uncomplicated malaria are treated with an ineffective antimalarial regimen, each episode of uncomplicated malaria is associated with a similar household cost, with an episode of vivax malaria costing around $9 \%$ of a households monthly income for the $60 \%$ of families making less than US\$500 per month (Chapter 2). Furthermore, an infectious bite from a mosquito can produce hypnozoites, which in high relapsing areas can result in eight or more 
recurrences per year in the absence of effective radical cure [11, 12]. Hence relapses have the potential to disrupt occupational and educational activities multiple times, with both short and long term consequences. In Brazil where nearly $70 \%$ of infections are due to $P$. vivax, it was demonstrated that the occurrence of one or more episodes of malaria impaired school performance [13]. Lastly, episodes of vivax malaria often happen in remote settings with poor healthcare services, rendering it difficult to access care.

The aim of this chapter is to collate information on the direct and indirect costs of illness due to $P$. vivax from a variety of endemic settings and match these with incidence estimates in order to provide the best possible estimate of the current global economic cost of illness to both healthcare providers and the households of patients. These estimates for the global costs due to vivax malaria are then used to conduct a thought experiment on the potential cost-benefit of implementing radical cure following a normal G6PD test result globally.

\subsection{Methods}

\subsubsection{Summary}

Global cost estimates were calculated for a base case, which reflects current practices for the diagnosis and treatment for $P$. vivax clinical cases in countries where it is endemic, and two alternative scenarios. These explore the potential cost-benefit of a global policy change to radical cure with 14 day primaquine in those who test G6PD normal. The first scenario assumes that radical cure is very effective and that a high investment is required to achieve adherence through supervised therapy. The second scenario does not include the costs of ensuring adherence and therefore assumes that the effectiveness is low. Key assumptions for the base case estimate are found in Table 6.1. 
Table 6.1: Key assumptions used in the base case global cost estimates.

\begin{tabular}{|c|c|c|}
\hline Parameter & Details & Source \\
\hline \multicolumn{3}{|l|}{ "COUNTRY-LEVEL } \\
\hline $\begin{array}{l}P . \text { vivax episode } \\
\text { estimates }\end{array}$ & $\begin{array}{l}\text { Generated by MAP for } 47 \text { countries. Listed in } \\
\text { Table } 6.2 \text { by whether or not they sought treatment } \\
\text { for their illness episode. }\end{array}$ & {$[14]$} \\
\hline $\begin{array}{l}\text { Percent of cases } \\
\text { receiving a malaria test }\end{array}$ & $\begin{array}{l}\text { Taken from the World Malaria Report. Listed in } \\
\text { Table 6.2. }\end{array}$ & {$[15]$} \\
\hline $\begin{array}{l}\text { Percent of cases } \\
\text { diagnosed by RDT }\end{array}$ & $\begin{array}{l}\text { Taken from the World Malaria Report. Listed in } \\
\text { Table 6.2. }\end{array}$ & {$[15]$} \\
\hline $\begin{array}{l}\text { Treatment of blood } \\
\text { stage infections }\end{array}$ & $\begin{array}{l}\text { Taken from the World Malaria Report. For } \\
\text { countries where } P \text {. vivax treatment was not listed, } \\
\text { the treatment for uncomplicated } P \text {. falciparum } \\
\text { was used. Listed in Table } 6.2 \text {. }\end{array}$ & {$[15]$} \\
\hline $\begin{array}{l}\text { Implementation of } \\
\text { G6PD screening }\end{array}$ & $\begin{array}{l}\text { Taken from MMV country prioritisation for } \\
\text { tafenoquine. Listed in Table } 6.2 \text {. }\end{array}$ & MMV \\
\hline $\begin{array}{l}\text { Use of radical cure with } \\
\text { primaquine }\end{array}$ & $\begin{array}{l}\text { Taken from MMV country prioritisation for } \\
\text { tafenoquine. Listed in Table } 6.2 \text {. }\end{array}$ & MMV \\
\hline $\begin{array}{l}\text { Percent ineligible } \\
\text { for primaquine }\end{array}$ & $\begin{array}{l}\text { Those younger than six months, pregnant or } \\
\text { lactating. Listed in Table } 6.2 \text {. }\end{array}$ & {$[16]$} \\
\hline $\begin{array}{l}\text { Percent with G6PD } \\
\text { deficiency }\end{array}$ & $\begin{array}{l}\text { G6PD deficiency for males and females with } \\
<30 \% \text { enzyme activity in general population that } \\
\text { is eligible for primaquine. These estimates do not } \\
\text { adjust for the protective effect of } P \text {. vivax and so } \\
\text { may be overestimates. Listed in Table } 6.2 \text {. }\end{array}$ & {$[17]$} \\
\hline
\end{tabular}

\section{REGIONAL}

Mean number of productive days lost

\section{Unit costs}

\section{GLOBAL}

Primaqune use

Primaqune use
Percent cases that are
severe in those seeking
treatment

The cost of a primaquine prescription was included for $90 \%$ of patients in all countries listed as prescribing primaquine.

Productivity losses to patients and their carers. These were extrapolated out by WHO region. For Southeast Asia and Western Pacific, the mean of Indonesia and Vietnam was used. Regional extrapolations from available data. See Table 6.3.
[18] for South

America and

Chapter 5 for all others

[19, 20, 21, 22, 23] and Chapter 5

Assumption

A systematic review of 140 studies with 53,164
$P$. vivax patients found a pooled prevalence of
severe anaemia of $2 \%$ (95\% CI: $1.3-2.8)$.

$\mathrm{MMV}=$ Medicines for Malaria Venture 


\subsubsection{Cases of vivax malaria}

The number of annual episodes of $P$. vivax were derived from those generated by the Malaria Atlas Project (MAP), which adjust country-level reports of clinical cases according to treatment-seeking rates and reporting completeness [14]. These estimates do not distinguish between new infections and relapses. Case estimates were available for all countries that reported P. vivax cases in 2015 [15] plus six additional countries that did not report cases (Democratic Republic of the Congo, Gabon, Madagascar, Somalia, Sudan and Tajikistan). Cases were adjusted for treatment-seeking rates at public facilities to adjust for those cases that would be missed by the national reporting system (i.e. those who did not seek treatment or sought treatment through private providers). Estimates of treatment seeking at any facility were also used to inform the lower bound of clinical cases; these assumed that any fevers that sought treatment were caused by malaria [25]. Here, treatment-seeking behaviour was categorized according to whether patients sought treatment with any provider (including public or private healthcare providers, pharmacies or shops) or did not seek treatment outside of their own home.

\subsubsection{Usual care for vivax malaria}

For those seeking treatment at healthcare providers, the proportion of malaria cases diagnosed by RDT and microscopy and drugs prescribed for the treatment of P. vivax blood stage infections were taken from the 2016 World Malaria Report [15]. For countries in Africa that did not report vivax-specific drug treatments, it was assumed that these countries did not distinguish between species and that $P$. vivax patients were treated with the drugs listed for uncomplicated confirmed P. falciparum. The use of G6PD screening and prescription of radical cure at the country level was taken from the Medicines for Malaria Venture (MMV) country prioritisation, which are listed in Table 6.2. Malaysia was the only country that regularly tests for G6PD deficiency before treatment, and the cost of a FST was added for each case. The cost of G6PD testing 
was not included in the base case scenario for any other country. For the 36 countries that prescribed primaquine, the cost of a prescription was included for $90 \%$ of the population in order to exclude those who are under the age of six months, lactating or presumed pregnant. It is also assumed that primaquine effectiveness is only $15 \%$ when prescribed without supervision [26]. Assumptions about diagnostic tests, treatment, days lost to illness and case estimates by country are shown in Table 6.2.

\subsubsection{Cost data}

Costs were collected in local currencies when possible, inflated to 2016 using GDP deflators [27] and then converted to US\$ using the exchange rate for mid-2016 [28]. When GDP deflators were missing for one or more years, the GDP deflator from the US was used instead. Costs to patients and healthcare providers were taken from the IMPROV study (Chapter 5) and three other locations reviewed by White et al. [29]: two in Brazil [20, 30] and one in Afghanistan [21]. These three studies provided data on diagnostic tests. The study in Afghanistan presented cost data for both low and high transmission areas: the mean of these were used for Eastern Mediterranean \& Europe regions [21]. A literature review with the aim of updating White and colleagues [29] with more recent studies found no further relevant cost data sources (Chapter

1). Transportation costs for Brazil were taken from a clinical trial on tafenoquine [18]. When country-specific data were not available, costs were applied by WHO region; Table 6.3 summarizes these costs.

Overhead treatment costs for P. vivax were taken from WHO-CHOICE [31] and supplemented with drug costs for a $60 \mathrm{~kg}$ adult from the International Medical Products Price Guide [23]. The cost of a primary care health centre without beds was used for the cost of outpatient visits and the cost per bed day in a primary-level hospital was used for inpatient visits [31]. Visit costs were not available for French Guiana, North Korea or Somalia, and in these cases the costs from neighbouring countries were used instead (Suriname, Cambodia and Ethiopia, respectively). It was assumed that $2 \%$ of patients who sought treatment had severe malaria [24] and therefore required three 
Table 6.2: Case estimates, mean number of productive days lost by patients and their carers, usual care for vivax malaria, ineligibility for primaquine, and G6PD deficiency $(<30 \%$ activity $)$ in those who are eligible for primaquine.

\begin{tabular}{|c|c|c|c|c|c|c|c|c|c|c|}
\hline \multirow[t]{2}{*}{ Country } & \multicolumn{2}{|c|}{ Number of cases [14] } & \multirow{2}{*}{$\begin{array}{l}\text { Productive } \\
\text { days lost } \\
{[19] \text { \& }} \\
\text { Ch. } 5\end{array}$} & \multicolumn{7}{|c|}{ Diagnosis \& Treatment } \\
\hline & $\begin{array}{l}\text { Did not } \\
\text { seek } \\
\text { treatment }\end{array}$ & $\begin{array}{l}\text { Sought } \\
\text { any } \\
\text { treatment }\end{array}$ & & $\begin{array}{l}\% \text { received } \\
\text { malaria } \\
\text { test }[15]\end{array}$ & $\begin{array}{l}\text { \% diag- } \\
\text { nosed by } \\
\text { RDT }[15]\end{array}$ & $\begin{array}{l}\text { Blood } \\
\text { stage } \\
\text { drug [15] }\end{array}$ & $\begin{array}{l}\text { PQ } \\
\text { used? }{ }^{\dagger}\end{array}$ & $\begin{array}{l}\text { Routine } \\
\text { G6PD } \\
\text { testing? }^{\dagger}\end{array}$ & $\begin{array}{l}\% \text { not } \\
\text { eligible for } \\
\text { PQ }[17]\end{array}$ & $\begin{array}{l}\% \text { G6PD } \\
\text { deficient } \\
{[16]^{*}}\end{array}$ \\
\hline Afghanistan & 125,934 & 213,180 & 3.5 & $30 \%$ & $16 \%$ & CQ & Yes & No & $4.9 \%$ & $13 \%$ \\
\hline Bangladesh & 787 & 931 & 5.9 & $100 \%$ & $76 \%$ & CQ & Yes & No & $3.0 \%$ & $6 \%$ \\
\hline Belize & 3 & 10 & 1.1 & $100 \%$ & $0 \%$ & CQ & Yes & No & $3.7 \%$ & $3 \%$ \\
\hline Bhutan & 9 & 26 & 5.9 & $100 \%$ & $19 \%$ & CQ & Yes & No & $3.4 \%$ & $10 \%$ \\
\hline Bolivia & 5,035 & 7,020 & 1.1 & $100 \%$ & $0 \%$ & CQ & Yes & No & $4.1 \%$ & $0 \%$ \\
\hline Brazil & 231,335 & 158,795 & 1.1 & $100 \%$ & $2 \%$ & CQ & Yes & No & $3.5 \%$ & $8 \%$ \\
\hline Cambodia & 3,315 & 23,414 & 5.9 & $100 \%$ & $78 \%$ & DHP & No & No & $4.0 \%$ & $25 \%$ \\
\hline China & 15 & 59 & 5.9 & $99 \%$ & $0 \%$ & CQ & Yes & No & $2.3 \%$ & $8 \%$ \\
\hline Colombia & 13,264 & 18,833 & 1.1 & $92 \%$ & $7 \%$ & CQ & Yes & No & $2.5 \%$ & $8 \%$ \\
\hline $\begin{array}{l}\text { Democratic Republic } \\
\text { of the Congo }\end{array}$ & 116,405 & 143,942 & 5.9 & $100 \%$ & $84 \%$ & ASAQ & No & No & $5.1 \%$ & $8 \%$ \\
\hline East Timor & 8 & 24 & 1.1 & $100 \%$ & $0 \%$ & CQ & Yes & No & $3.6 \%$ & $7 \%$ \\
\hline Ecuador & 200 & 620 & 1.1 & $100 \%$ & $0 \%$ & CQ & Yes & No & $2.9 \%$ & $5 \%$ \\
\hline El Salvador & 1 & 3 & 5.7 & $100 \%$ & $0 \%$ & CQ & No & No & $5.0 \%$ & $7 \%$ \\
\hline Eritrea & 6,142 & 6,743 & 5.7 & $80 \%$ & $57 \%$ & ASAQ & No & No & $5.2 \%$ & $2 \%$ \\
\hline Ethiopia & $2,193,480$ & 802,980 & 1.1 & $86 \%$ & $0 \%$ & CQ & Yes & No & $3.2 \%$ & $1 \%$ \\
\hline French Guiana & 140 & 314 & 5.7 & $100 \%$ & $37 \%$ & CQ & No & No & $4.3 \%$ & $22 \%$ \\
\hline Gabon & 1,635 & 3,571 & 1.1 & $11 \%$ & $15 \%$ & ASAQ & Yes & No & $4.2 \%$ & $4 \%$ \\
\hline Guatemala & 5,381 & 10,182 & 1.1 & $100 \%$ & $19 \%$ & CQ & Yes & No & $2.7 \%$ & $5 \%$ \\
\hline Guyana & 5,873 & 11,840 & 1.1 & $100 \%$ & $0 \%$ & CQ & Yes & No & $3.5 \%$ & $5 \%$ \\
\hline Honduras & 1,613 & 2,722 & 5.9 & $100 \%$ & $1 \%$ & CQ & Yes & No & $6.5 \%$ & $14 \%$ \\
\hline India & 980,869 & $3,045,940$ & 6.6 & $100 \%$ & $0 \%$ & CQ & Yes & No & $4.7 \%$ & $12 \%$ \\
\hline Indonesia & 78,859 & 402,964 & 3.5 & $100 \%$ & $0 \%$ & DHP & No & No & $3.1 \%$ & $21 \%$ \\
\hline Iran & 190 & 706 & 5.9 & $100 \%$ & $42 \%$ & $\mathrm{CQ}$ & No & No & $4.4 \%$ & $29 \%$ \\
\hline Laos & 19,156 & 39,701 & 5.7 & $100 \%$ & $88 \%$ & CQ & No & No & $5.4 \%$ & $37 \%$ \\
\hline Madagascar & 36,550 & 38,045 & 5.9 & $99 \%$ & $99 \%$ & ASAQ & Yes & No & $2.6 \%$ & $13 \%$ \\
\hline Malaysia & 35 & 97 & 1.1 & $100 \%$ & $0 \%$ & CQ & Yes & FST & $4.1 \%$ & $2 \%$ \\
\hline Mexico & 175 & 635 & 5.9 & $100 \%$ & $0 \%$ & CQ & Yes & No & $2.8 \%$ & $10 \%$ \\
\hline Myanmar & 22,722 & 39,352 & 5.9 & $100 \%$ & $92 \%$ & CQ & Yes & No & $3.4 \%$ & $9 \%$ \\
\hline Nepal & 2,755 & 6,432 & 1.1 & $9 \%$ & $39 \%$ & CQ & Yes & No & $3.1 \%$ & $2 \%$ \\
\hline Nicaragua & 1,017 & 2,086 & 5.9 & $100 \%$ & $0 \%$ & CQ & Yes & No & $3.3 \%$ & $0 \%$ \\
\hline North Korea & 3,426 & 8,378 & 5.7 & $95 \%$ & $0 \%$ & CQ & No & No & $6.1 \%$ & $37 \%$ \\
\hline Pakistan & 635,613 & $2,720,491$ & 3.5 & $5 \%$ & $32 \%$ & CQ & Yes & No & $3.9 \%$ & $27 \%$ \\
\hline Panama & 197 & 599 & 1.1 & $100 \%$ & $0 \%$ & CQ & Yes & No & $2.9 \%$ & $1 \%$ \\
\hline Papua New Guinea & 49,118 & 104,130 & 5.9 & $54 \%$ & $78 \%$ & $\mathrm{AL}$ & Yes & No & $4.7 \%$ & $13 \%$ \\
\hline Peru & 36,892 & 78,698 & 1.1 & $100 \%$ & $0 \%$ & CQ & Yes & No & $3.4 \%$ & $0 \%$ \\
\hline Philippines & 869 & 915 & 5.9 & $100 \%$ & $3 \%$ & CQ & Yes & No & $5.2 \%$ & $43 \%$ \\
\hline Solomon Islands & 7,415 & 16,253 & 3.5 & $47 \%$ & $38 \%$ & $\mathrm{AL}$ & Yes & No & $5.8 \%$ & $5 \%$ \\
\hline Somalia & 496 & 649 & 5.9 & $53 \%$ & $100 \%$ & $\mathrm{AL}$ & Yes & No & $1.4 \%$ & $0 \%$ \\
\hline South Korea & 172 & 634 & 5.9 & $100 \%$ & $0 \%$ & CQ & Yes & No & $3.6 \%$ & $4 \%$ \\
\hline Sudan & 56,780 & 84,751 & 3.5 & $53 \%$ & $0 \%$ & $\mathrm{AL}$ & Yes & No & $3.7 \%$ & $28 \%$ \\
\hline Suriname & 47 & 111 & 1.1 & $100 \%$ & $8 \%$ & CQ & Yes & No & $2.6 \%$ & $1 \%$ \\
\hline Tajikistan & 0 & 1 & 3.5 & $100 \%$ & $0 \%$ & CQ & No & No & $4.5 \%$ & $1 \%$ \\
\hline Thailand & 1,062 & 2,328 & 5.9 & $96 \%$ & $0 \%$ & CQ & Yes & No & $1.5 \%$ & $24 \%$ \\
\hline Vanuatu & 139 & 312 & 5.9 & $61 \%$ & $96 \%$ & $\mathrm{AL}$ & Yes & No & $4.9 \%$ & $13 \%$ \\
\hline Venezuela & 78,382 & 214,873 & 1.1 & $100 \%$ & $0 \%$ & CQ & Yes & No & $2.7 \%$ & $15 \%$ \\
\hline Vietnam & 4,281 & 6,102 & 3.7 & $48 \%$ & $0 \%$ & CQ & Yes & No & $2.6 \%$ & $15 \%$ \\
\hline Yemen & 1,046 & 1,775 & 3.5 & $72 \%$ & $45 \%$ & $\mathrm{CQ}$ & No & No & $4.6 \%$ & $8 \%$ \\
\hline
\end{tabular}

$\mathrm{PQ}=$ primaquine; $\mathrm{CQ}=$ chloroquine; $\mathrm{DHP}=$ dihydroartemisinin-piperaquine; ASAQ $=$ artesunate-amodiaquine; $\mathrm{AL}=$ artemether-lumefantrine; $\mathrm{FST}=$ fluorescent spot test. $\dagger$ Taken from MMV country prioritisation for tafenoquine

* These are calculated for the population that is not pregnant, lactating or under the age of six months. 
Table 6.3: Unit costs and assumptions by WHO region (2016 US\$).

\begin{tabular}{|c|c|c|c|c|c|}
\hline Region & Africa & Americas & Asia $*$ & $\begin{array}{l}\text { Eastern } \\
\text { Mediterranean } \\
\& \text { Europe }\end{array}$ & Assumptions \\
\hline FST & - & - & 20.76 & - & Chapter 5 \\
\hline G6PD RDT & 1.18 & 3.48 & 1.43 & 2.03 & $\begin{array}{l}\text { Americas from }[22] \text {, Chapter } 5 \text { for Asia } \\
\text { (Vietnam) and for Africa, Eastern } \\
\text { Mediterranean \& Europe is average of } \\
\text { other regions }\end{array}$ \\
\hline Chloroquine & 0.30 & 0.30 & 0.30 & 0.30 & [23] Only available buyer price \\
\hline Dihydroartemisinin-piperaquine & 2.80 & 2.80 & 2.80 & 2.80 & [23] Median supplier price \\
\hline Artesunate-amodiaquine & 0.35 & 0.35 & 0.35 & 0.35 & [23] Only available supplier price \\
\hline Artesunate-lumefantrine & 0.79 & 0.79 & 0.79 & 0.79 & [23] Only available buyer price \\
\hline Primaquine & 0.85 & 1.42 & 1.13 & 1.13 & $\begin{array}{l}{[23] \text { Africa and Americas taken from }} \\
\text { regional prices then averaged } \\
\text { for other regions. }\end{array}$ \\
\hline Treatment seeking & 0.10 & 0.65 & 1.45 & 0.40 & $\begin{array}{l}\text { Chapter } 5 \text { (Americas is average from } \\
\text { other regions). }\end{array}$ \\
\hline
\end{tabular}

* Includes both South-East Asia and Western Pacific regions.

days of inpatient care [32]. Provider costs were obtained from the public sector; these were assumed to be representative of the true costs of illness for private providers (i.e. excluding profits). The cost of deaths due to $P$. vivax were excluded.

Table 6.4 describes the assumptions for individuals seeking treatment as compared to those who did not seek treatment for their $P$. vivax episode. For those seeking treatment, direct costs to the patient included the cost of transport to the healthcare facility in addition to payments for treatment and transport for any other treatment seeking (Chapter 5).

\subsubsection{Costs of productivity losses}

Indirect costs included the cost of the days when patients were unable to attend to usual activities due to illness and also the days when their caregiver was required to stop doing usual activities to look after the patient (Chapter 5). The time lost by 
Table 6.4: How household cost categories were applied by treatment-seeking behaviour.

\begin{tabular}{|c|c|c|}
\hline Cost category & $\begin{array}{l}\text { Seeking treatment at any } \\
\text { provider }\end{array}$ & Not seeking treatment \\
\hline $\begin{array}{l}\text { Cost of diagnosis and } \\
\text { treatment }\end{array}$ & $\begin{array}{l}\text { Costs for diagnosis and treatment } \\
\text { by country policy. }\end{array}$ & Not included. \\
\hline Transport costs & $\begin{array}{l}\text { Cost for the patient to get to and } \\
\text { from the healthcare provider. }\end{array}$ & Not included. \\
\hline $\begin{array}{l}\text { Cost of previous treatment } \\
\text { seeking }\end{array}$ & $\begin{array}{l}\text { Costs to the household for } \\
\text { treatment and transport for any } \\
\text { treatment taking before diagnosis. }\end{array}$ & Not included. \\
\hline $\begin{array}{l}\text { Indirect cost of time off } \\
\text { usual activities }\end{array}$ & $\begin{array}{l}\text { Cost per day using GDP per capita } \\
\text { per day. Patient and carer days } \\
\text { taken from country-level data when } \\
\text { possible. When not possible, taken } \\
\text { from average of studies from WHO } \\
\text { region. }\end{array}$ & $\begin{array}{l}\text { Cost per day using GDP per capita } \\
\text { per day. Patient and carer days } \\
\text { taken from country-level data when } \\
\text { possible. When not possible, taken } \\
\text { from average of studies from WHO } \\
\text { region. }\end{array}$ \\
\hline
\end{tabular}

patients and caregivers were valued equally, and indirect costs were included for all cases, regardless of age or employment status. When days of illness data were not available for a country, these were applied by WHO region (Table 6.2). For Asia, this was the weighted average of Indonesia and Vietnam by number of patients in each country. The days of illness were multiplied by the GDP per capita per day to calculate the indirect costs per episode. GDP per capita estimates were taken from The World Bank for all but seven countries [33]. For six countries (East Timor, Eritrea, Iran, Papua New Guinea and Vietnam), the GDP per capita estimate was taken from the International Monetary Fund [34]; and the GDP per capita was estimated to be US $\$ 1,300$ for North Korea [35].

\subsubsection{Low and high cost bounds}

Low and high cost bounds were calculated for the base case estimate. The lower and upper case bounds were provided by MAP [14] utilising estimation methods from Cibulskis and colleagues [25]. The 95\% CIs for treatment seeking were also provided by MAP [14], and the 95\% CIs for hospitalisations due to severe disease were taken from a systematic review [24]. The low cost bound combined the lower case estimate, lower $95 \%$ CI on treatment seeking and low $95 \%$ CI for hospitalisations due to severe 
disease. Likewise, the high cost bound used the higher case estimate, higher 95\% CI on treatment seeking and high 95\% CI for hospitalisations.

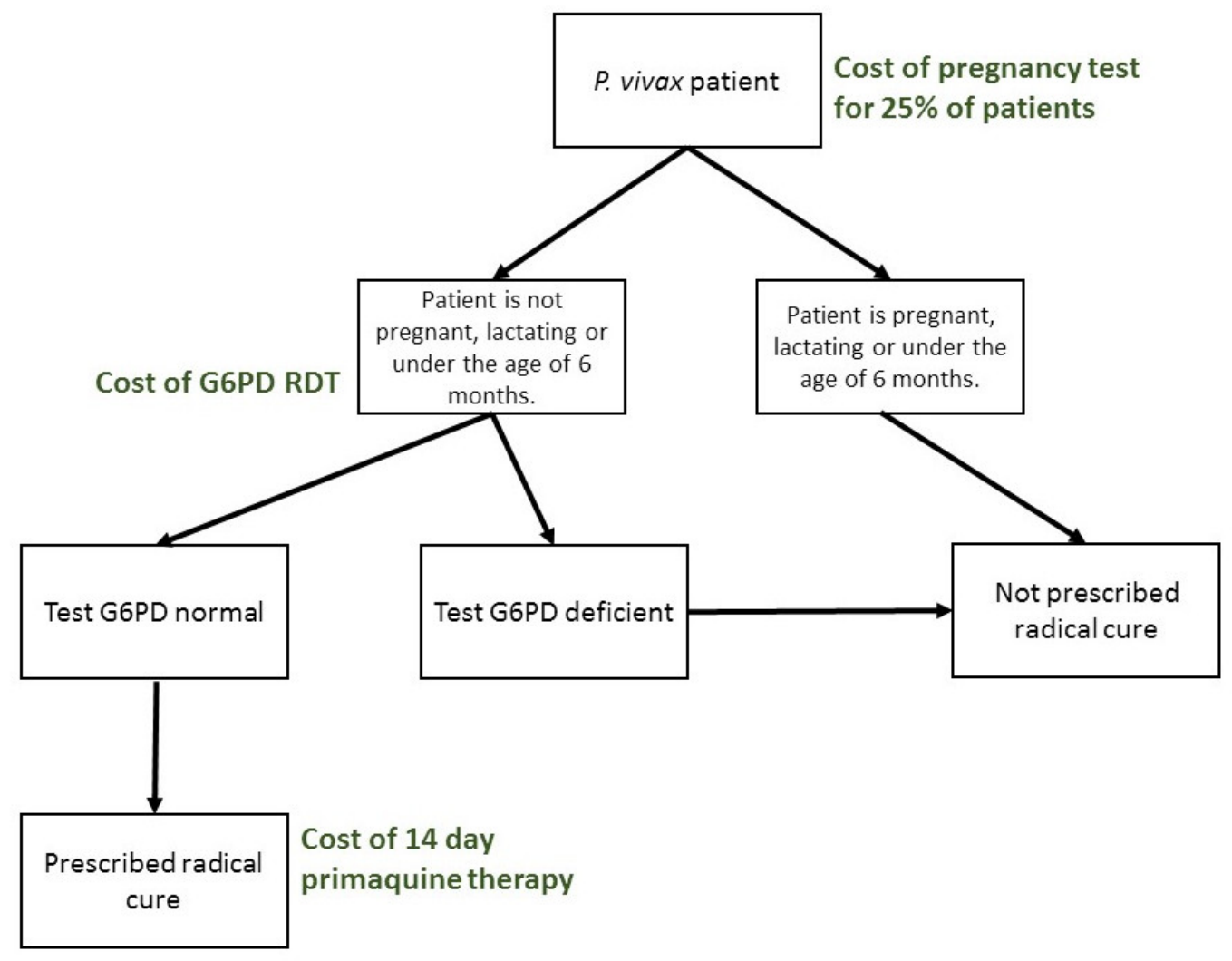

Figure 6.1: Flow diagram of $P$. vivax patient case management in the scenario analyses.

\subsubsection{Global cost-benefit of implementing G6PD testing with highly effective radical cure}

A scenario analysis explored the potential impact of implementing G6PD screening in those who sought treatment followed by supervised primaquine therapy over 14 days, following a G6PD normal test result. A cost-benefit analysis was then carried out for this Highly effective radical cure scenario as compared to the base case estimates, including the health benefits in terms of increased productivity and direct costs averted due to lower relapse episodes. Figure 6.1 shows the flow of $P$. vivax patients and how costs were applied. A proportion of the population are ineligible for primaquine due to them being under the age of six months, pregnant or lactating; these proportions are listed by country in Table 6.2 [16]. To ensure that pregnant women did not receive primaquine, 
the cost of providing a pregnancy test (Chapter 4) for $25 \%$ of the population was included. After excluding those who were ineligible for primaquine, the cost of a G6PD RDT was applied to the rest of the population. Individuals who are confirmed to be G6PD normal would receive 14 day primaquine under supervision to ensure full adherence, and those identified as deficient would not receive radical cure due to their risk of primaquine-induced haemolysis. These costs replaced any pre-existing costs of screening and primaquine in the base case analysis. The percent of the population with G6PD deficiency ( $<30 \%$ activity) in those eligible for primaquine (i.e. not pregnant, lactating or under six months old) is also listed in Table 6.2 [17].

The following assumptions were made for those who were prescribed primaquine:

1. All patients were adherent to their regimens. The cost of supervised treatment in order to achieve this was estimated to be 3.25 healthcare worker days (1/4th day for 13 days) with time valuated using the GDP per capita per day. This represents the additional investment necessary to achieve complete adherence. (Other lower cost options, such as text message reminders are available, but adherence is also likely be lower.)

2. Primaquine was assumed to be effective at preventing relapses in $85 \%$ of the individuals who completed treatment $[12,36]$.

3. For the $85 \%$ of patients who received an effective dose of primaquine, a reduction in relapses resulted in cost savings due to relapse episodes averted. Table 6.5 lists the clinical incidence per person year observed for countries grouped into nine zoogeographical zones from Battle et al., which was assumed to be the relapse incidence following primary infections [37]. For each infection treated with an effective dose of primaquine, the relapse incidence in Table 6.5 was multiplied by the mean cost per treatment-seeking episode using the base case scenario to calculate the cost savings per relapse prevented.

4. Since countries currently prescribing primaquine were assumed to have an effectiveness of $15 \%$ through unsupervised therapy [26], it was assumed that the base 
Table 6.5: Countries and relapse incidence among cases per person year observed by zoogeographical zone from Battle and colleagues [37].

\begin{tabular}{llc}
\hline $\begin{array}{l}\text { Zoogeographical } \\
\text { zones }\end{array}$ & Countries & $\begin{array}{c}\text { Relapse incidence per } \\
\text { person year observed }\end{array}$ \\
\hline North America & Mexico & 1.7 \\
Central America & $\begin{array}{l}\text { Belize, Guatemala, Honduras, Nicaragua, Panama, } \\
\text { El Salvador } \\
\text { Bolivia, Brazil, Columbia, Ecuador, French Guiana, Guyana, }\end{array}$ & 0.9 \\
South America & $\begin{array}{l}\text { Peru, Suriname, Venezuela } \\
\text { Democratic Republic of the Congo, Eritrea, Ethiopia, Gabon, }\end{array}$ & 4.0 \\
Sub-Saharan Africa & Madagascar, Somalia, Sudan, Yemen & 0.8 \\
Monsoon Asia & India, Iran, Pakistan & 0.2 \\
South East Asia & Bangladesh, Bhutan, Indonesia, Cambodia, Laos, Myanmar, \\
Northern Europe \& Asia & Malaysia, Nepal, Philippines, Thailand, East Timor, Vietnam & 3.1 \\
Melanesia & Papua New Guinea, Solomon Islands, Vanuatu & 0.5 \\
\hline
\end{tabular}

case estimates inherently reflected those prevented relapses. Accordingly, the implementation of supervised primaquine would reduce cases in only $70 \%$ of those who received the prescription instead of $85 \%$.

\subsubsection{Global cost-benefit of radical cure with low adherence}

The Low adherence to radical cure scenario investigated what would happen if primaquine was administered unsupervised with a lower level of adherence assumed. This scenario differs from the Highly effective radical cure scenario in the following ways:

1. The costs of supervised primaquine therapy were excluded.

2. It was assumed that for countries that currently do not prescribe primaquine, introduction of an unsupervised regimen would only reduce relapses in $15 \%$ of those prescribed primaquine [26]. Again, this was equivalent to the relapse incidence found in Table 6.5. In countries where primaquine is already used, no additional benefits were seen in terms of cases averted. 


\subsection{Results}

\subsubsection{Global cost burden estimates}

The total global cost of vivax malaria in 47 countries was estimated to be US $\$ 330$ million per year (Table 6.6). Figure 6.2 displays a global map of the costs. The lower bound was US $\$ 205$ million while the upper bound was US\$459 million. Of the base case estimates, $80 \%$ (US $\$ 263$ million) was attributable to indirect patient costs, $12 \%$ (US $\$ 41$ million) to healthcare provider costs and $8 \%$ (US\$26 million) to direct costs to the patient. India carried the highest cost burden with US $\$ 137$ million, accounting for $42 \%$ of the total global cost, of which $80 \%$ (US $\$ 111$ million) were due to indirect costs. Other high cost countries were Pakistan (US\$64 million), Indonesia (US\$37 million), Ethiopia (US\$36 million), Brazil (US\$12 million) and Venezuela (US\$12 million). Tajikistan had the lowest country cost at US\$18 for its one case (Table 6.6).

\subsubsection{Global cost-benefit of implementing effective G6PD testing and radical cure}

In the Highly effective radical cure scenario, the additional provider costs of G6PD screening in conjunction with administration of a supervised 14 day primaquine regimen were calculated to be US $\$ 113$ million, which would be offset by a cost savings of US $\$ 34$ million due to relapses prevented (Table 6.7 and Figure 6.4B). In total, the net cost to providers was US $\$ 121$ million, a nearly three-fold increase as compared with their base case costs (Figure 6.3). Despite these global increases in healthcare provider costs, four countries (Bolivia, Cambodia, Columbia and Solomon Islands) had net cost savings to the provider due to implementation of G6PD screening followed by radical cure (Table 6.7). Most of the cost savings were seen at the patient level, where total costs dropped by US\$124 million (47\%) to US\$165 million. Overall, the Highly effective radical cure scenario resulted in a reduction in costs due to vivax malaria treatment of US\$45 million (14\%) to US\$285 million (Figure 6.3). 
Table 6.6: Base case global cost estimates by country (2016 US\$).

\begin{tabular}{|c|c|c|c|c|c|c|}
\hline \multirow{2}{*}{ Country } & \multirow{2}{*}{$\begin{array}{l}\text { Provider } \\
\text { costs }\end{array}$} & \multicolumn{2}{|c|}{ Patient costs } & \multirow{2}{*}{ Total costs } & \multirow{2}{*}{$\begin{array}{l}\text { Low } \\
\text { bound }\end{array}$} & \multirow{2}{*}{$\begin{array}{l}\text { High } \\
\text { bound }\end{array}$} \\
\hline & & Direct & Indirect & & & \\
\hline Afghanistan & 950,631 & 682,176 & $1,826,776$ & $3,459,583$ & $1,954,160$ & $4,652,628$ \\
\hline Bangladesh & 3,235 & 3,353 & 37,526 & 44,114 & 20,851 & 68,661 \\
\hline Belize & 157 & 18 & 190 & 365 & 257 & 488 \\
\hline Bhutan & 169 & 92 & 1,568 & 1,830 & 1,340 & 2,337 \\
\hline Bolivia & 77,164 & 12,648 & 112,803 & 202,616 & 126,329 & 285,707 \\
\hline Brazil & $1,440,678$ & 286,124 & $10,170,028$ & $11,896,829$ & $5,041,109$ & $19,088,287$ \\
\hline Cambodia & 146,552 & 84,291 & 545,447 & 776,290 & 626,294 & 934,013 \\
\hline China & 681 & 211 & 7,668 & 8,561 & 8,515 & 8,613 \\
\hline Colombia & 392,272 & 33,935 & 561,583 & 987,790 & 641,448 & $1,363,177$ \\
\hline $\begin{array}{l}\text { Democratic Republic } \\
\text { Of the Congo }\end{array}$ & 289,615 & 273,489 & $1,807,218$ & $2,370,322$ & $1,503,599$ & $3,260,443$ \\
\hline East Timor & 106 & 86 & 1,149 & 1,341 & 1,054 & 1,631 \\
\hline Ecuador & 9,949 & 1,117 & 14,737 & 25,803 & 17,665 & 35,035 \\
\hline El Salvador & 24 & 6 & 56 & 86 & 63 & 110 \\
\hline Eritrea & 20,756 & 12,812 & 165,628 & 199,196 & 94,363 & 310,318 \\
\hline Ethiopia & $1,550,348$ & $1,525,662$ & $33,072,053$ & $36,148,063$ & $10,712,909$ & $62,238,153$ \\
\hline French Guiana & 4,482 & 566 & 50,426 & 55,473 & 29,688 & 82,593 \\
\hline Gabon & 46,848 & 6,785 & 583,686 & 637,320 & 450,102 & 831,136 \\
\hline Guatemala & 133,165 & 18,347 & 194,492 & 346,003 & 210,707 & 499,494 \\
\hline Guyana & 120,718 & 21,334 & 237,891 & 379,942 & 244,746 & 527,286 \\
\hline Honduras & 30,017 & 4,905 & 30,851 & 65,773 & 46,112 & 86,917 \\
\hline India & $15,855,861$ & $10,965,385$ & $110,611,817$ & $137,433,063$ & $80,381,482$ & $195,581,742$ \\
\hline Indonesia & $4,426,936$ & $1,450,669$ & $31,105,873$ & $36,983,479$ & $25,112,593$ & $49,563,239$ \\
\hline Iran & 8,737 & 2,258 & 34,008 & 45,004 & 44,419 & 45,671 \\
\hline Laos & 181,807 & 142,922 & $2,225,591$ & $2,550,321$ & $1,502,555$ & $3,645,747$ \\
\hline Madagascar & 96,550 & 72,286 & 467,503 & 636,339 & 352,326 & 935,028 \\
\hline Malaysia & 3,756 & 349 & 20,195 & 24,301 & 17,782 & 31,099 \\
\hline Mexico & 14,129 & 1,145 & 20,023 & 35,297 & 25,384 & 46,663 \\
\hline Myanmar & 132,338 & 141,669 & $1,271,820$ & $1,545,827$ & $1,015,362$ & $2,094,345$ \\
\hline Nepal & 10,841 & 23,155 & 107,703 & 141,698 & 86,063 & 201,295 \\
\hline Nicaragua & 18,341 & 3,759 & 20,121 & 42,222 & 29,601 & 55,816 \\
\hline North Korea & 39,785 & 30,161 & 246,595 & 316,541 & 183,469 & 460,003 \\
\hline Pakistan & $8,038,548$ & $8,705,572$ & $47,249,127$ & $63,993,247$ & $55,417,789$ & $72,911,019$ \\
\hline Panama & 14,528 & 1,079 & 32,798 & 48,405 & 35,249 & 62,985 \\
\hline Papua New Guinea & 454,528 & 374,868 & $6,375,537$ & $7,204,933$ & $4,734,435$ & $9,752,043$ \\
\hline Peru & $1,272,823$ & 141,801 & $2,106,023$ & $3,520,647$ & $2,329,525$ & $4,852,976$ \\
\hline Philippines & 6,614 & 3,295 & 84,627 & 94,537 & 41,776 & 149,787 \\
\hline Solomon Islands & 96,004 & 58,512 & 762,754 & 917,270 & 602,677 & $1,247,188$ \\
\hline Somalia & 1,734 & 2,076 & 4,766 & 8,575 & 2,894 & 15,583 \\
\hline South Korea & 27,640 & 2,282 & 280,565 & 310,488 & 307,197 & 314,249 \\
\hline Sudan & 689,194 & 271,204 & $3,277,569$ & $4,237,968$ & $2,697,750$ & $5,851,658$ \\
\hline Suriname & 1,735 & 200 & 3,089 & 5,024 & 3,525 & 6,675 \\
\hline Tajikistan & 7 & 3 & 7 & 18 & 18 & 18 \\
\hline Thailand & 27,086 & 8,382 & 321,838 & 357,306 & 236,057 & 483,015 \\
\hline Vanuatu & 2,322 & 1,121 & 20,713 & 24,157 & 16,021 & 32,670 \\
\hline Venezuela & $4,554,376$ & 387,168 & $6,734,572$ & $11,676,116$ & $7,896,058$ & $16,011,003$ \\
\hline Vietnam & 23,095 & 21,966 & 230,045 & 275,105 & 164,461 & 390,291 \\
\hline Yemen & 13,478 & 5,682 & 26,794 & 45,953 & 32,709 & 59,948 \\
\hline Total & $41,230,793$ & $25,786,923$ & $263,047,634$ & $330,065,351$ & $204,991,498$ & $459,056,632$ \\
\hline
\end{tabular}



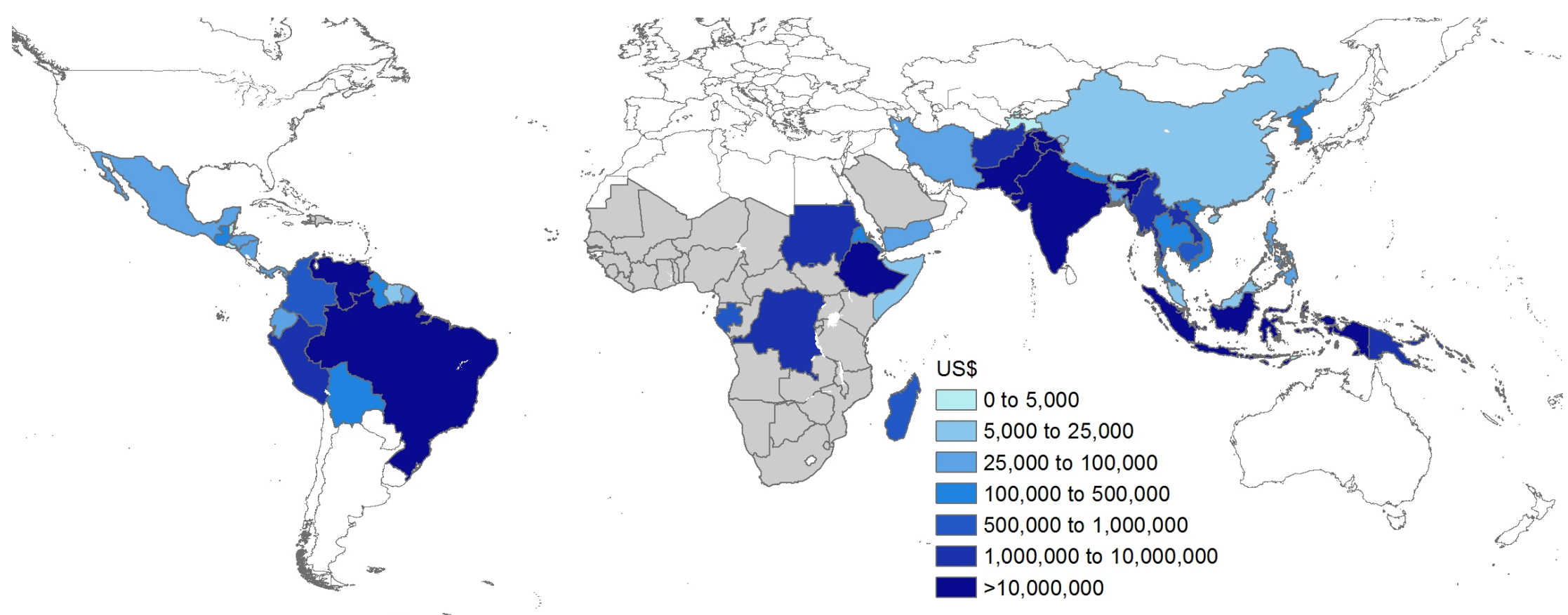

Figure 6.2: Global map of $P$. vivax economic cost burden in the base case (2016 US\$). Countries in grey are thought to be $P$. vivax endemic not enough information is available to generate estimates. 


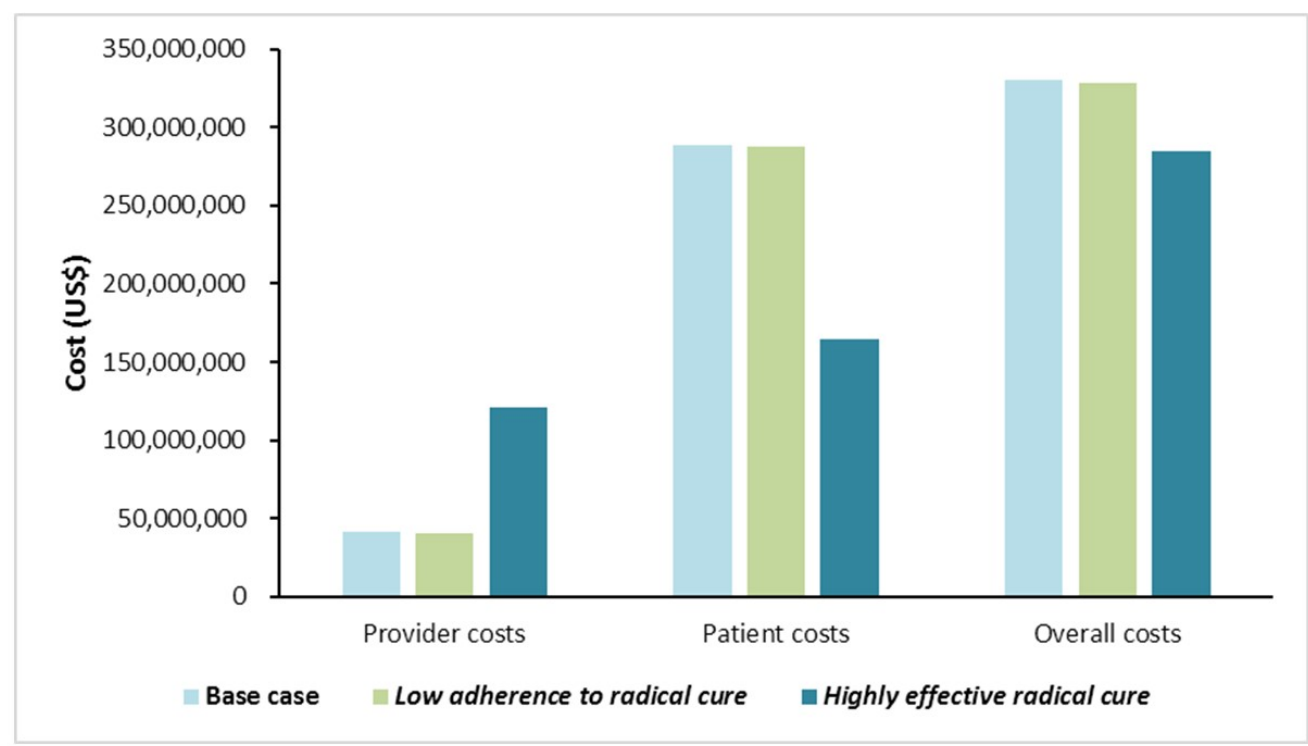

Figure 6.3: Provider, patient and overall cost comparison between the global base case estimate and the Low adherence to radical cure and Highly effective radical cure scenarios (2016 US\$).

\subsubsection{Global cost-benefit of radical cure with low adherence}

The Low adherence to radical cure scenario had a much smaller impact with total costs similar to the base case estimates at US\$328 million (Figures $6.3 \& 6.4 \mathrm{~A}$ ). Since an additional investment to improve adherence through observed therapy was not included, the overall costs to the healthcare provider were reduced by US\$0.3 million (1\%) to US $\$ 41$ million (Table 6.8). Around $81 \%$ (US\$0.3 million) of these cost savings were due to the reduction in cases; the additional savings were due to the prevention of primaquine prescriptions in G6PD deficient patients. Thirteen countries had reductions in healthcare provider costs due in this scenario as compared to the base case scenario. At the patient level, the Low adherence to radical cure scenario resulted in modest cost savings of US\$1 million (6\%) to US\$287 million.

\subsection{Discussion}

The cost figures presented in the base case scenario bring together country-level epidemiological data with recently available disease-specific cost data to present the best available evidence on the current global economic burden of $P$. vivax malaria. The 
Table 6.7: Results of the Highly effective radical cure scenario as compared to the base case estimates (2016 US\$). The net benefit to the healthcare providers is the sum of the additional screening and treatment costs and the reduction in provider costs due to relapses prevented.

\begin{tabular}{|c|c|c|c|c|c|c|}
\hline Country & $\begin{array}{l}\text { Base case } \\
\text { total costs }\end{array}$ & $\begin{array}{l}\text { Additional costs } \\
\text { for screening \& } \\
\text { treatment }\end{array}$ & $\begin{array}{l}\text { Provider cost } \\
\text { savings due to } \\
\text { averted cases }\end{array}$ & $\begin{array}{l}\text { Overall change } \\
\text { in provider } \\
\text { costs }\end{array}$ & $\begin{array}{l}\text { Household cost } \\
\text { savings due to } \\
\text { averted cases }\end{array}$ & Net benefit* \\
\hline Afghanistan & $3,459,583$ & 874,463 & 270,384 & 604,079 & 520,659 & $-83,420$ \\
\hline Bangladesh & 44,114 & 10,304 & 6,295 & 4,009 & 46,104 & 42,095 \\
\hline Belize & 365 & 397 & 97 & 301 & 99 & -202 \\
\hline Bhutan & 1,830 & 561 & 316 & 245 & 2,329 & 2,084 \\
\hline Bolivia & 202,616 & 186,240 & 205,977 & $-19,736$ & 209,101 & 228,837 \\
\hline Brazil & $11,896,829$ & $10,861,059$ & $3,572,391$ & $7,288,668$ & $10,974,097$ & $3,685,429$ \\
\hline Cambodia & 776,290 & 209,144 & 272,119 & $-62,975$ & $1,043,713$ & $1,106,688$ \\
\hline China & 8,561 & 3,828 & 210 & 3,618 & 2,429 & $-1,189$ \\
\hline Colombia & 987,790 & 873,929 & 982,896 & $-108,967$ & 910,682 & $1,019,649$ \\
\hline $\begin{array}{l}\text { Democratic Republic } \\
\text { of the Congo }\end{array}$ & $2,370,322$ & 410,776 & 112,579 & 298,197 & 494,712 & 196,515 \\
\hline East Timor & 1,341 & 438 & 239 & 198 & 2,123 & 1,925 \\
\hline Ecuador & 25,803 & 29,608 & 24,963 & 4,645 & 30,771 & 26,125 \\
\hline El Salvador & 86 & 120 & 15 & 106 & 30 & -76 \\
\hline Eritrea & 199,196 & 49,177 & 12,187 & 36,990 & 58,412 & 21,422 \\
\hline Ethiopia & $36,148,063$ & $5,372,359$ & 955,889 & $4,416,470$ & $6,404,985$ & $1,988,515$ \\
\hline French Guiana & 55,473 & 98,749 & 11,992 & 86,756 & 94,815 & 8,059 \\
\hline Gabon & 637,320 & 173,285 & 23,178 & 150,107 & 201,452 & 51,345 \\
\hline Guatemala & 346,003 & 345,419 & 80,842 & 264,577 & 88,389 & $-176,187$ \\
\hline Guyana & 379,942 & 436,174 & 312,434 & 123,740 & 466,775 & 343,035 \\
\hline Honduras & 65,773 & 52,819 & 18,280 & 34,538 & 14,784 & $-19,755$ \\
\hline India & $137,433,063$ & $37,033,390$ & $2,020,176$ & $35,013,214$ & $12,057,185$ & $-22,956,029$ \\
\hline Indonesia & $36,983,479$ & $10,721,289$ & $7,927,212$ & $2,794,077$ & $49,181,818$ & $46,387,742$ \\
\hline Iran & 45,004 & 24,850 & 1,289 & 23,561 & 5,350 & $-18,212$ \\
\hline Laos & $2,550,321$ & 600,587 & 265,535 & 335,052 & $2,401,336$ & $2,066,284$ \\
\hline Madagascar & 636,339 & 100,692 & 37,806 & 62,886 & 121,667 & 58,781 \\
\hline Malaysia & 24,301 & 6,897 & 6,772 & 125 & 27,304 & 27,179 \\
\hline Mexico & 35,297 & 43,839 & 15,502 & 28,337 & 18,482 & $-9,855$ \\
\hline Myanmar & $1,545,827$ & 390,452 & 247,145 & 143,307 & $1,770,325$ & $1,627,017$ \\
\hline Nepal & 141,698 & 36,916 & 20,440 & 16,476 & 185,824 & 169,348 \\
\hline Nicaragua & 42,222 & 38,022 & 11,489 & 26,533 & 10,827 & $-15,705$ \\
\hline North Korea & 316,541 & 94,514 & 13,157 & 81,357 & 67,855 & $-13,502$ \\
\hline Pakistan & $63,993,247$ & $24,236,502$ & 887,763 & $23,348,739$ & $5,191,279$ & $-18,157,461$ \\
\hline Panama & 48,405 & 69,867 & 9,201 & 60,666 & 16,317 & $-44,349$ \\
\hline Papua New Guinea & $7,204,933$ & $1,994,263$ & $1,184,088$ & 810,176 & $12,262,021$ & $11,451,846$ \\
\hline Peru & $3,520,647$ & $4,085,640$ & $3,420,526$ & 665,114 & $4,234,350$ & $3,569,236$ \\
\hline Philippines & 94,537 & 22,360 & 13,104 & 9,256 & 92,517 & 83,261 \\
\hline Solomon Islands & 917,270 & 149,633 & 161,288 & $-11,654$ & 978,284 & 989,938 \\
\hline Somalia & 8,575 & 2,256 & 843 & 1,412 & 2,324 & 912 \\
\hline South Korea & 310,488 & 152,863 & 9,300 & 143,562 & 95,171 & $-48,391$ \\
\hline Sudan & $4,237,968$ & $1,242,687$ & 259,434 & 983,253 & 840,899 & $-142,354$ \\
\hline Suriname & 5,024 & 6,187 & 4,667 & 1,520 & 6,370 & 4,850 \\
\hline Tajikistan & 18 & 10 & 3 & 7 & 4 & -3 \\
\hline Thailand & 357,306 & 91,625 & 43,437 & 48,188 & 367,930 & 319,743 \\
\hline Vanuatu & 24,157 & 6,515 & 5,979 & 536 & 39,757 & 39,222 \\
\hline Venezuela & $11,676,116$ & $12,100,628$ & $10,569,922$ & $1,530,707$ & $12,350,755$ & $10,820,049$ \\
\hline Vietnam & 275,105 & 97,946 & 40,833 & 57,113 & 277,858 & 220,745 \\
\hline Yemen & 45,953 & 15,631 & 7,855 & 7,776 & 13,138 & 5,362 \\
\hline Total & $330,081,139$ & $113,354,909$ & $34,048,047$ & $79,306,862$ & $124,183,409$ & $44,876,547$ \\
\hline
\end{tabular}

* Positive numbers indicate cost savings as compared to the base case estimate. 

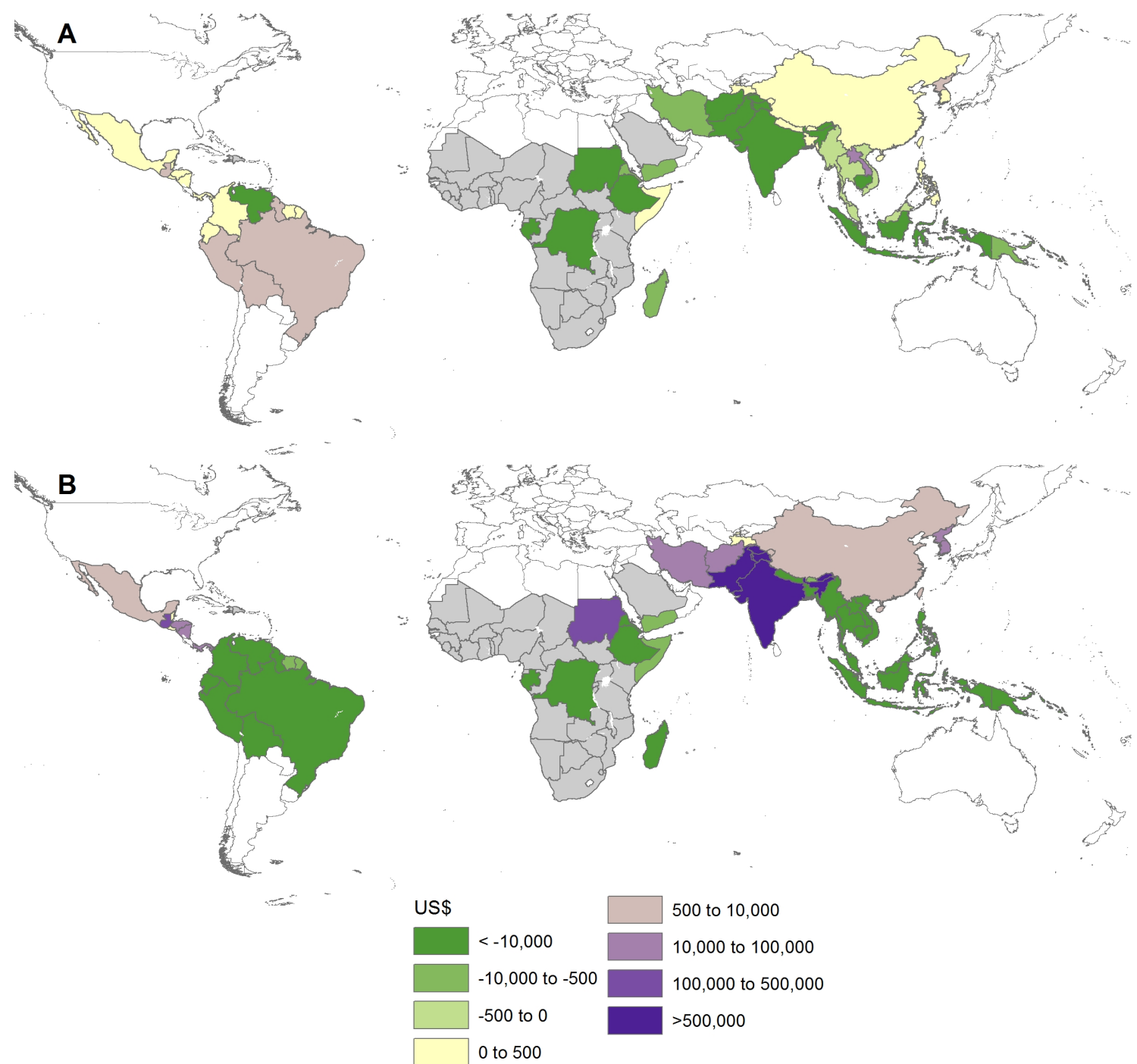

Figure 6.4: Global map of changes in the P. vivax cost burden in the scenario analyses as compared to the base case: A) Low adherence to radical cure and B) Highly effective radical cure scenario. Green signifies net benefits (i.e. cost savings) and purple indicates cost increases. Costs are in 2016 US $\$$. Countries in grey are thought to be $P$. vivax endemic, but not enough information is available to generate estimates. 
Table 6.8: Results of the Low adherence to radical cure scenario as compared to the base case estimates (2016 US\$). The net benefit to the healthcare providers is the sum of the additional screening and treatment costs and the reduction in provider costs due to relapses prevented.

\begin{tabular}{|c|c|c|c|c|c|c|}
\hline Country & $\begin{array}{l}\text { Base case } \\
\text { total costs }\end{array}$ & $\begin{array}{l}\text { Additional costs } \\
\text { for screening \& } \\
\text { treatment }\end{array}$ & $\begin{array}{l}\text { Provider cost } \\
\text { savings due to } \\
\text { averted cases }\end{array}$ & $\begin{array}{l}\text { Overall change } \\
\text { in provider } \\
\text { costs }\end{array}$ & $\begin{array}{l}\text { Household cost } \\
\text { savings due to } \\
\text { averted cases }\end{array}$ & Net benefit* \\
\hline Afghanistan & $3,459,583$ & $-11,419$ & - & $-11,419$ & - & 11,419 \\
\hline Bangladesh & 44,114 & 36 & - & 36 & - & -36 \\
\hline Belize & 365 & 4 & - & 4 & - & -4 \\
\hline Bhutan & 1,830 & 1 & - & 1 & - & -1 \\
\hline Bolivia & 202,616 & 735 & - & 735 & - & -735 \\
\hline Brazil & $11,896,829$ & 1,256 & - & 1,256 & - & $-1,256$ \\
\hline Cambodia & 776,290 & 19,607 & 48,021 & $-28,414$ & 184,185 & 212,598 \\
\hline China & 8,561 & 3 & - & 3 & - & -3 \\
\hline Colombia & 987,790 & 401 & - & 401 & - & -401 \\
\hline $\begin{array}{l}\text { Democratic Republic } \\
\text { of the Congo }\end{array}$ & $2,370,322$ & 75,658 & 19,867 & 55,791 & 87,302 & 31,511 \\
\hline East Timor & 1,341 & 26 & 42 & -17 & 375 & 391 \\
\hline Ecuador & 25,803 & 18 & - & 18 & - & -18 \\
\hline El Salvador & 86 & 4 & - & 4 & - & -4 \\
\hline Eritrea & 199,196 & 5,268 & 2,151 & 3,118 & 10,308 & 7,190 \\
\hline Ethiopia & $36,148,063$ & 657,672 & 168,686 & 488,985 & $1,130,292$ & 641,306 \\
\hline French Guiana & 55,473 & 37 & - & 37 & - & -37 \\
\hline Gabon & 637,320 & 2,366 & 4,090 & $-1,724$ & 35,550 & 37,275 \\
\hline Guatemala & 346,003 & 509 & - & 509 & - & -509 \\
\hline Guyana & 379,942 & 749 & - & 749 & - & -749 \\
\hline Honduras & 65,773 & 150 & - & 150 & - & -150 \\
\hline India & $137,433,063$ & $-254,628$ & - & $-254,628$ & - & 254,628 \\
\hline Indonesia & $36,983,479$ & $-18,119$ & - & $-18,119$ & - & 18,119 \\
\hline Iran & 45,004 & 634 & 227 & 406 & 944 & 538 \\
\hline Laos & $2,550,321$ & 31,798 & - & 31,798 & - & $-31,798$ \\
\hline Madagascar & 636,339 & 20,137 & 6,672 & 13,466 & 21,471 & 8,005 \\
\hline Malaysia & 24,301 & -23 & - & -23 & - & 23 \\
\hline Mexico & 35,297 & 59 & - & 59 & - & -59 \\
\hline Myanmar & $1,545,827$ & -162 & - & -162 & - & 162 \\
\hline Nepal & 141,698 & 36 & - & 36 & - & -36 \\
\hline Nicaragua & 42,222 & 193 & - & 193 & - & -193 \\
\hline North Korea & 316,541 & 838 & - & 838 & - & -838 \\
\hline Pakistan & $63,993,247$ & $-558,136$ & - & $-558,136$ & - & 558,136 \\
\hline Panama & 48,405 & 67 & - & 67 & - & -67 \\
\hline Papua New Guinea & $7,204,933$ & $-5,311$ & - & $-5,311$ & - & 5,311 \\
\hline Peru & $3,520,647$ & 8,926 & - & 8,926 & - & $-8,926$ \\
\hline Philippines & 94,537 & 53 & - & 53 & - & -53 \\
\hline Solomon Islands & 917,270 & $-6,283$ & - & $-6,283$ & - & 6,283 \\
\hline Somalia & 8,575 & 14 & - & 14 & - & -14 \\
\hline South Korea & 310,488 & 77 & - & 77 & - & -77 \\
\hline Sudan & $4,237,968$ & $-17,912$ & - & $-17,912$ & - & 17,912 \\
\hline Suriname & 5,024 & 16 & - & 16 & - & -16 \\
\hline Tajikistan & 18 & 3 & 0.1 & 3 & 1 & -2 \\
\hline Thailand & 357,306 & -335 & - & -335 & - & 335 \\
\hline Vanuatu & 24,157 & -18 & - & -18 & - & 18 \\
\hline Venezuela & $11,676,116$ & $-15,637$ & - & $-15,637$ & - & 15,637 \\
\hline Vietnam & 275,105 & -346 & - & -346 & - & 346 \\
\hline Yemen & 45,953 & 1,823 & 1,386 & 437 & 2,318 & 1,881 \\
\hline Total & $330,081,139$ & $-59,156$ & 251,143 & $-310,299$ & $1,472,745$ & $1,783,044$ \\
\hline
\end{tabular}

* Positive numbers indicate cost savings as compared to the base case estimate. 
alternative scenarios provide insights into how costs might be impacted in a world that embraces G6PD screening and radical cure prescription.

While it was possible to gather data about country-level policies, such as the percent receiving a malaria test (Table 6.1), it was more challenging to find country-specific cost data (Table 6.3). As a result, the WHO-CHOICE costs were used for the visit costs alongside regional extrapolations of vivax-specific costs. The $P$. vivax case estimates (Table 6.2) also have uncertainty linked to the quality of reporting in each country. The cases reported here, prior to adjusting for treatment seeking and reporting, are from the World Malaria Report [15]. For the majority of countries, these are the nationally reported estimates, and so are the best available evidence. The case estimates from the three countries with the highest numbers (Ethiopia, India and Pakistan) have been inflated from the nationally reported data to reflect reporting completeness. These inflations may have been too extreme in either direction, which would then impact the global costs.

The estimates in this chapter highlight the need to gather further data on the epidemiology and costs of $P$. vivax and can be updated as this data becomes available. While the various data issues design a great deal of uncertainty about the economic costs related to vivax malaria treatment, this is the first global estimate of the costs of vivax malaria illness that attempts to incorporate country-level estimates. The only previous estimate of $\$ 1.4-4.0$ billion was by Price et al., which combined global case estimates for clinical cases with regional costs from WHO-CHOICE and assumed a daily wage of $\$ 1.5$ for all patients [38]. These higher costs were the result of using case estimates that were ten-fold higher than those used here (106-313 million as compared to 13 million). Future work should include a PSA to investigate the uncertainty further, though choosing ranges and distributions necessary will be a challenging task. It is possible that if the global cost burden is reported without a nuanced discussion of the data limitations, then this information might be used to advocate for more or less investment in $P$. vivax treatment and control. Such actions would be misguided as the base case estimate provides information on the cost burden but does not indicate how 
resources should be allocated (i.e. cost-effectiveness).

These base case estimates were also a valuable opportunity to look at the potential cost-benefit of implementing G6PD screening before radical cure. Both alternative scenarios resulted in cost savings overall when both patient and healthcare provider costs were included. A surprising finding was that the majority of the cost savings in both scenarios were at the patient level due to the prevention of clinical relapses. This is due to the assumptions around productivity losses including the losses to individuals who do not seek treatment. Since the investment in G6PD screening and radical cure will likely be made by the healthcare providers, this finding could present a challenge in terms of financing. The Low adherence to radical cure scenario provides insight into what would happen without supervised treatment for radical cure: fewer cases were averted but modest decreases were seen in the healthcare provider costs due to primaquine prescriptions being limited to only those who could take it safely.

The Highly effective radical cure scenario told a different story with provider costs increasing three-fold in order to screen for pregnancy and G6PD deficiency and prescribe 14 day supervised primaquine therapy. This increase of US\$80 million in healthcare provider expenses in the Highly effective radical cure scenario would represent only $3 \%$ of the US\$2.7 billion spent on malaria control and elimination in 2016 [39]. It should be noted that while India and Pakistan have high cost and case estimates, they have a very low relapse incidence at 0.2 per year, which had a large impact on the overall cost-benefit results. If these two countries were excluded, the cost savings in this scenario would be more considerable due to the exclusion of the high costs of supervision and the low benefits of radical cure in these settings. This illustrates how it will be more challenging for G6PD deficiency followed by radical cure to be cost-effective in long latency settings. For countries with long latency relapse patterns, a transmission model with a longer time horizon would be more appropriate to capture the long-term benefits of relapses prevented; however, this would introduce uncertainty since little data are available on relapses that occur beyond the first year. Furthermore, the alternative scenarios relied on relapse incidence data that divided countries into zoographical zones. Within 
countries like India a variety of relapse patterns can occur, so the cost-effectiveness of radical cure might also vary within the country. The results presented here do not reflect the aforementioned nuances.

Given that little operational research has been done on how to provide supervised primaquine therapy or what costs would be associated with this, the reality of moving toward global radical cure is likely to lie somewhere between these two scenarios until a safer drug or one with a much shorter regimen is available. Furthermore, it is likely that interventions to increase adherence will need to be tailored to individual settings. Supervision could be via mobile phone, education programs, or less frequent visits than the daily supervision that was costed in the Highly effective radical cure scenario. While the introduction of single dose tafenoquine will alleviate the issue with adherence relative to a 14 day primaquine regimen, many individuals will not be prescribed this drug, including those with G6PD enzyme activity less than $70 \%$, which will exclude more people than the current $30 \%$ cut off currently recommended for primaquine. These individuals will need to take 14 day primaquine or the regimen of eight weekly doses in order to achieve radical cure. In more remote settings where healthcare staff are unable to interpret a quantitative test for G6PD deficiency, primaquine is likely to continue to be the standard of care. Until a drug that is safe for individuals with G6PD deficiency is available, the design and implementation of interventions to improve adherence to primaquine regimens will continue to be critical to malaria elimination efforts.

An issue that arose in generating the global cost burden was the valuation of productivity losses, or costs associated with inability to work or participate in leisure activities due to illness or death. While no attempt was made to capture the cost of death, an attempt was made to valuate the time lost to illness, which was possible. While it is accepted that time is a limited resource with opportunity costs, economic evaluation methods do not agree on whether it should be included or how to do so. Chapter 5 reported days that patients and any carers were unable to participate in usual activities, but it also reported that the majority of individuals were not in paid employment. Vivax malaria disproportionately affects the poor, with the majority of 
individuals in Chapter 5 reporting that they missed school or another unpaid activity, such as housework or subsistence farming. In an attempt to capture those losses, the use of one GDP per capita per day was used for each day missed. This is equivent to $1 / 365$ th of a DALY if a willingness to pay threshold of one GDP per capita was used. The $P$. vivax case estimates did not indicate cases by age (i.e. children vs. adults), so productivity losses were equivalent for both children and adults. If childrens' time had been valuated at a lower value than adults, then the overall costs would have decreased; however, this helps to ameliorate their educational losses that would otherwise be unaccounted for. In order for educational losses to be included properly in these estimates, further studies need to be designed to measure these losses. These would need to be followed by a consensus on best practice for valuating the loss.

As demonstrated in Chapters 2 \& 5, vivax malaria often disproportionately affects the poor, which has implications in terms of productivity losses and equity. If the reported wages from those who had vivax had been available and used here, the global costs would have been lower. If these figures were used to generate ICERs which were then compared with other diseases, it could exacerbate inequity between those who are rich and poor. This further supports the use of GDP per capita per day as a shadow price for productivity losses. When considering the scenario analyses, the use of G6PD screening before radical cure could further exacerbate inequity if a safe option is not available for remote settings with higher concentrations of poor households where community health workers have limited training and are not able to deploy G6PD tests. This would likely result in a drop in $P$. vivax episodes in richer areas while the disease burden in poorer areas remained stagnate.

The scenario analyses use a cost model that calculates the benefits of radical cure without accounting for its potential to prevent onward transmission of $P$. vivax infections; consequently the results underestimate the benefits of radical cure and are less robust than what would be possible with a dynamic transmission model. In terms of the impact on cost estimates, it is important to note that the increased investment in radical cure was calculated using the number of episodes in the base case estimate; 
however, the benefit of radical cure would result in fewer cases requiring this increased investment, meaning that the necessary investment has likely been overestimated. While the costs were calculated over one year, the benefits would stretch out further into the future (i.e. a patient treated on day 365 of that year would have benefits in terms of decreased relapses over the following year). Furthermore, the time to first relapse from a primary infection can take longer than one year, but our relapse estimates use only the mean number of relapses in one year [37]. Data on longer-term outcomes are sparse. Decreasing the transmission potential is one of the only means currently available to protect those who cannot safely receive primaquine treatment.

The costs of the scenario analyses may have been underestimated due to the exclusion of the cost of primaquine-induced haemolysis and the cost of any Haemocue ${ }^{\mathrm{TM}}$ tests required. Primaquine-induced haemolysis was not included in any of the estimates due to uncertainty around its frequency and severity [40]. In countries that are prescribing primaquine without first testing for G6PD deficiency, it is possible that haemolysis already occurs. In settings where primaquine is not being prescribed, the alternative scenarios might increase the incidence of haemolysis further where imperfect diagnostic accuracy and human error could lead to some individuals with G6PD deficiency incorrectly being prescribed primaquine therapy; though it is hoped that some of this would be prevented with supervised therapy. Other limitations are related to the availability of data. While the vivax-specific cost data were extrapolated by region, it is likely that these costs will vary considerably from country to country. Public provider costs were applied to all individuals seeking treatment. While this reflects the true cost of treatment, patient costs would likely be higher when seeking treatment at private providers due to treatment fees.

In addition to the aforementioned valuation of productivity losses, the costs of the scenario analyses may have been overestimated due to a number of factors. Vivax malaria cases were not stratified by age and so all treatment costs are for adults. It was assumed that a change in policy would change both the private and the public sectors, potentially overestimating both the costs and the benefits. While public policy 
does not mandate its adoption by private providers, Chapter 3 showed that private providers may also shift their behaviour, particularly if patients know where to get efficacious treatment. It is also possible that screening would not be cost-effective in areas where G6PD deficiency has a low prevalence or mild severity in the general population, meaning that only the costs of primaquine therapy should be included in the Highly effective radical cure scenario.

In conclusion, the economic costs due to vivax malaria treatment and productivity losses are substantial. The scenario analyses indicate that utilising G6PD screening to safely prescribe radical cure in those who are G6PD normal would be cost-beneficial globally, but may not be economically justifiable in low-relapse settings. 


\subsection{Declarations}

\subsubsection{Acknowledgments}

I am grateful to Katherine Battle and Rosalind Howes for helpful discussions and providing epidemiological data. I also thank Katherine Battle for producing Figures 6.2 $\& 6.4$. 


\section{References for Chapter 6}

[1] Anstey NM, Russell B, Yeo TW, Price RN. The pathophysiology of vivax malaria. Trends Parasitol. 2009;25(5):220-7.

[2] Douglas NM, Pontororing GJ, Lampah DA, Yeo TW, Kenangalem E, Poespoprodjo JR, et al. Mortality attributable to Plasmodium vivax malaria: a clinical audit from Papua, Indonesia. BMC Med. 2014;12:217. doi:10.1186/s12916-014-0217-z.

[3] Poespoprodjo JR, Fobia W, Kenangalem E, Lampah DA, Hasanuddin A, Warikar N, et al. Vivax Malaria: A Major Cause of Morbidity in Early Infancy. Clin Infect Dis. 2009;48(12):1704-1712.

[4] Moore KA, Simpson JA, Wiladphaingern J, Min AM, Pimanpanarak M, Paw MK, et al. Influence of the number and timing of malaria episodes during pregnancy on prematurity and small-for-gestational-age in an area of low transmission. BMC Med. 2017;15(1):117. doi:10.1186/s12916-017-0877-6.

[5] Bhatt S, Weiss DJ, Cameron E, Bisanzio D, Mappin B, Dalrymple U, et al. The effect of malaria control on Plasmodium falciparum in Africa between 2000 and 2015. Nature. 2015;526(7572):207-211. doi:10.1038/nature15535.

[6] Thriemer K, Ley B, Bobogare A, Dysoley L, Alam MS, Pasaribu AP, et al. Challenges for achieving safe and effective radical cure of Plasmodium vivax: a round table discussion of the APMEN Vivax Working Group. Malar J. 2017;16(1):141. doi:10.1186/s12936-017-1784-1.

[7] World Health Organization. World malaria report 2015.; 2015.

[8] Douglas NM, Simpson JA, Phyo AP, Siswantoro H, Hasugian AR, Kenangalem E, et al. Gametocyte dynamics and the role of drugs in reducing the transmission potential of Plasmodium vivax. J Infect Dis. 2013;208(5):801-12. doi:10.1093/infdis/jit261. 
[9] Gallup JL, Sachs JD. The economic burden of malaria. Am J Trop Med Hyg. 2001;64(1-2 Suppl):85-96.

[10] Sachs J, Malaney P. The economic and social burden of malaria. Nature. 2002;415(6872):680-5. doi:10.1038/415680a.

[11] Chu CS. Factors affecting Plasmodium vivax recurrence. PhD Thesis, The Open University. 2015;.

[12] Abreha T, Hwang J, Thriemer K, Tadesse Y, Girma S, Melaku Z, et al. Comparison of artemether-lumefantrine and chloroquine with and without primaquine for the treatment of Plasmodium vivax infection in Ethiopia: A randomized controlled trial. PLoS Med. 2017;14(5):e1002299. doi:10.1371/journal.pmed.1002299.

[13] Vitor-Silva S, Reyes-Lecca RC, Pinheiro TR, Lacerda MV. Malaria is associated with poor school performance in an endemic area of the Brazilian Amazon. Malar J. 2009;8:230. doi:10.1186/1475-2875-8-230.

[14] Battle KE, Weiss DJ, Lucas T, Nguyen M, Bhatt S, Cameron E, et al. The global burden of Plasmodium vivax malaria: 2000-2016. (In preparation);.

[15] World Health Organization. World malaria report 2016.; 2016.

[16] Baird JK, Battle KE, Howes RE. Primaquine ineligibility in anti-relapse therapy of Plasmodium vivax malaria: the problem of G6PD deficiency and cytochrome P-450 2D6 polymorphisms. Malar J. 2018;17(1):42. doi:10.1186/s12936-018-2190-z.

[17] Howes RE, Piel FB, Patil AP, Nyangiri OA, Gething PW, Dewi M, et al. G6PD deficiency prevalence and estimates of affected populations in malaria endemic countries: a geostatistical model-based map. PLoS Med. 2012;9(11):e1001339. doi:10.1371/journal.pmed.1001339.

[18] GlaxoSmithKline. Ph 2B/3 Tafenoquine (TFQ) Study in Prevention of Vivax Relapse. National Library of Medicine (US). 2011;Available from: 
https://clinicaltrials.gov/ct2/show/NCT01376167 NLM Identifier: NCT 01376167(Accessed: 2017 August 17).

[19] GlaxoSmithKline. Study to Assess the Incidence of Hemolysis, Safety, and Efficacy of Tafenoquine (SB-252263, WR238605) Versus Primaquine in Subjects With Plasmodium Vivax Malaria. National Library of Medicine (US). 2014;Available from: https://clinicaltrials.gov/ct2/show/NCT02216123 NLM Identifier: NCT02216123(Accessed: 2017 August 17).

[20] Peixoto HM, Brito MA, Romero GA, Monteiro WM, de Lacerda MV, de Oliveira MR. Cost-effectiveness analysis of rapid diagnostic tests for G6PD deficiency in patients with Plasmodium vivax malaria in the Brazilian Amazon. Malar J. 2016;15(1):82. doi:10.1186/s12936-016-1140-x.

[21] Hansen KS, Grieve E, Mikhail A, Mayan I, Mohammed N, Anwar M, et al. Cost-effectiveness of malaria diagnosis using rapid diagnostic tests compared to microscopy or clinical symptoms alone in Afghanistan. Malar J. 2015;14:217. doi:10.1186/s12936-015-0696-1.

[22] Brito MA, Peixoto HM, Almeida AC, Oliveira MR, Romero GA, Moura-Neto JP, et al. Validation of the rapid test Carestart ${ }^{\mathrm{TM}}$ G6PD among malaria vivax-infected subjects in the Brazilian Amazon. Rev Soc Bras Med Trop. 2016;49(4):446-55. doi:10.1590/0037-8682-0134-2016.

[23] MSH (Management Sciences for Health). International Medical Products Price Guide, 2015 Edition; 2017. Available from: http://mshpriceguide.org/en/home/.

[24] Rahimi BA, Thakkinstian A, White NJ, Sirivichayakul C, Dondorp AM, Chokejindachai W. Severe vivax malaria: a systematic review and meta-analysis of clinical studies since 1900. Malar J. 2014;13:481. doi:10.1186/1475-2875-13-481. 
[25] Cibulskis RE, Aregawi M, Williams R, Otten M, Dye C. Worldwide incidence of malaria in 2009: estimates, time trends, and a critique of methods. PLoS Med. 2011;8(12):e1001142. doi:10.1371/journal.pmed.1001142.

[26] Douglas NM, Poespoprodjo JR, Patriani D, Malloy MJ, Kenangalem E, Sugiarto $\mathrm{P}$, et al. Unsupervised primaquine for the treatment of Plasmodium vivax malaria relapses in southern Papua: A hospital-based cohort study. PLoS Med. 2017;14(8):e1002379. doi:10.1371/journal.pmed.1002379.

[27] The World Bank. Inflation, GDP deflator (annual \%). 2016;Available from: www . data.worldbank.org/indicator/NY.GDP.DEFL.KD.ZG(Accessed: 08 September 2017).

[28] XE. Current and Historical Rate Tables. 2016;Available from: http: //www . xe.com/currencytables/?from=USD\&date=2016-07-01 (Accessed: 8 September 2017).

[29] White MT, Yeung S, Patouillard E, Cibulskis R. Costs and Cost-Effectiveness of Plasmodium vivax Control. Am J Trop Med Hyg. 2016;95(6 Suppl):52-61. doi:10.4269/ajtmh.16-0182.

[30] de Oliveira MR, Giozza SP, Peixoto HM, Romero GA. Cost-effectiveness of diagnostic for malaria in Extra-Amazon Region, Brazil. Malar J. 2012;11:390. doi:10.1186/1475-2875-11-390.

[31] World Health Organisation. WHO-CHOICE unit cost estimates for service delivery. 2011;Available from: http://www.who.int/choice/ cost-effectiveness/inputs/health_service/en/(Accessed: 02 February 2016).

[32] Botto-Menezes C, Bardaji A, Dos Santos Campos G, Fernandes S, Hanson K, Martinez-Espinosa FE, et al. Costs Associated with Malaria in Pregnancy in the Brazilian Amazon, a Low Endemic Area Where Plasmodium vivax Predominates. PLoS Negl Trop Dis. 2016;10(3):e0004494. doi:10.1371/journal.pntd.0004494. 
[33] The World Bank. GDP per capita (current US\$). 2016;Available from: http://data.worldbank.org/indicator/NY.GDP.PCAP.CD(Accessed: 23 August 2017).

[34] International Monetary Fund. World Economic Outlook Database, October 2017. Available from: http:

//wwwimforg/external/pubs/ft/weo/2017/02/weodata/indexaspx;(Accessed: 24 October 2017).

[35] Lee J. North Korea's Economy Is Growing at Its Fastest Pace Since 1999. Bloomberg; Available from https://www . bloomberg.com/news/articles/2017-07-21/ north-korea-s-economy-rebounds-from-drought-amid-missile-focus.

[36] Nelwan EJ, Ekawati LL, Tjahjono B, Setiabudy R, Sutanto I, Chand K, et al. Randomized trial of primaquine hypnozoitocidal efficacy when administered with artemisinin-combined blood schizontocides for radical cure of Plasmodium vivax in Indonesia. BMC Med. 2015;13:294. doi:10.1186/s12916-015-0535-9.

[37] Battle KE, Karhunen MS, Bhatt S, Gething PW, Howes RE, Golding N, et al. Geographical variation in Plasmodium vivax relapse. Malar J. 2014;13:144. doi:10.1186/1475-2875-13-144.

[38] Price RN, Tjitra E, Guerra CA, Yeung S, White NJ, Anstey NM. Vivax malaria: neglected and not benign. Am J Trop Med Hyg. 2007;77(6 Suppl):79-87.

[39] World Health Organization. World malaria report 2017.; 2017.

[40] Ashley EA, Recht J, White NJ. Primaquine: the risks and the benefits. Malar J. 2014;13:418. doi:10.1186/1475-2875-13-418. 


\section{Chapter 7}

\section{Discussion}

\subsection{Summary}

While $P$. vivax was once considered a neglected disease [1], recent gains in understanding its long-term impact on health coupled with the rise of $P$. vivax in countries targeting $P$. falciparum elimination have brought it to the fore [2]. This thesis further demonstrates that the burden of vivax malaria is high through a range of studies in a variety of settings, beginning with evidence that the cost to households was similar for both vivax and falciparum malaria (Chapter 2). This burden can be ameliorated via improvements in the care provided in formal sector, as shown by the large beneficial impact on health and treatment-seeking behaviour in Chapter 3. The biggest obstacle to radical cure is the risk of haemolysis in those who have G6PD deficiency. The results of the decision model in Chapter 4 demonstrated that treatment strategies using G6PD RDTs and radical cure were cost-effective. Accordingly, this strategy should also be rolled out, and Chapter $\mathbf{6}$ showed how this could be cost-beneficial from a global perspective drawing on cost data specific to $P$. vivax from Chapter 5. Novel treatment options for radical cure will become available in the near future alongside the newly developed point-of-care G6PD tests necessary to enable their use. This is a promising time for vivax malaria research, and a comprehensive understanding of the economic consequences of alternative options for the management of vivax malaria is 
critical for optimising malaria control strategies in Asia, South and Mesoamerica and the Horn of Africa. An ongoing theme was the paucity of data available relating to the costs and cost-effectiveness of $P$. vivax. Accordingly, the aim of this thesis was to describe the economic burden of $P$. vivax malaria and the costs and cost-effectiveness of screening and treatment strategies for its optimal management. While each of the previous chapters has its own focus, they come together to provide a comprehensive view of the economics of $P$. vivax treatment.

\subsection{Empirical findings}

The first objective was to describe the costs due to vivax malaria episodes from the societal and healthcare provider perspectives. The first important finding on costs came from the healthcare facility exit surveys in Chapter 2, which found that costs incurred during $P$. vivax or P. falciparum malaria episodes were similar when patients were managed in public facilities. Additional information on household costs due to $P$. vivax episodes are presented in Chapter 5, including the costs of illness incurred up to 13 days following diagnosis. The cost estimates for vivax malaria are also expanded in Chapter 5 to include the provider costs per episode and the additional costs required to provide G6PD screening before prescribing high-dose primaquine. Importantly, the cost data in Chapter 5 only included vivax malaria patients (i.e. no P. falciparum patients); this is the first study to report these for healthcare provider costs [3]. Another key finding was that screening for G6PD deficiency via RDTs consistently cost less than the fluorescent spot test (FST). These data expand what is known about the cost of $P$. vivax treatment considerably. As demonstrated in Chapters 1 \& $\mathbf{6}$, cost data is still unavailable for many countries.

The second objective was to describe the treatment-seeking behaviour and provider compliance following a change in antimalarial policy requiring blood test confirmation of malaria and treatment with DHP for malaria due to any species in Timika, Indonesia, where a large proportion of cases are due to vivax malaria. Chapters $2 \& 3$ highlighted how changes in policy have the potential to lead to meaningful improvements in both 
provider and patient behaviour. Firstly, household surveys showed a ten-fold decrease in the proportion of individuals reporting fever in the preceding month (from $42 \%$ to $4 \%$ ); and those individuals with fever were more likely to seek treatment at a public provider as compared to seeking treatment at private providers only. Both public and private providers were more likely to adhere to local antimalarial guidelines with an increase in the percentage of patients receiving blood tests for malaria and prescriptions of the correct antimalarial drug regimen. The improvement in providers adhering to the guidelines was most apparent in the private sector. Furthermore, a significant increase in treatment with 14 day primaquine was observed in those who reported being diagnosed with $P$. vivax (from $9 \%$ to $63 \%$ ).

Importantly, the proportion of household members with symptomatic malaria dropped significantly, from $32 \%$ to $6 \%$, indicating that the changes in patient behaviour were likely a result of patients receiving more effective treatment and patients being aware of the benefits of the new treatments. The universal policy of ACT for all species of malaria also led to a significant decrease in repeat visits to healthcare providers and thus a significant fall in the indirect costs per fever episode. While these findings include both $P$. vivax and P. falciparum and the species may not have been reported correctly due to recall bias, a large percentage of cases in this area are due to $P$. vivax. Accordingly, these chapters demonstrate that it is important to ensure effective $P$. vivax treatment and that policy decisions have an impact on the behaviour of $P$. vivax patients and healthcare providers.

The next objective was to evaluate the cost-effectiveness of screening for G6PD deficiency before treatment with supervised primaquine therapy. Chapter 4 demonstrates that on the Thailand-Myanmar border, screening for G6PD deficiency prior to administration of a supervised 14 day primaquine regimen resulted in substantial health benefits for the patients. The costs were similar when compared to a strategy of no radical cure, and cost savings were seen in the comparison with a strategy where primaquine is given without screening. While our findings should be relevant for settings that are similar to the Thailand-Myanmar border, the epidemiology of P. vivax, G6PD 
deficiency and costs vary greatly across endemic areas. In order to address these other settings, an online tool was created where the model parameters can be adjusted to reflect other settings, and the cost-effectiveness of radical cure following G6PD screening is reported for those parameter values.

The final objective was to describe what is currently known about the global economic cost burden due to P. vivax malaria, the potential costs associated with a worldwide policy of screening before giving radical cure, and the potential cost-benefit associated with a reduction in vivax episodes. In Chapter 6, the provider and household costs presented in Chapter $\mathbf{5}$ were extrapolated and combined with data available from other cost studies to estimate the global economic cost burden due to $P$. vivax malaria. The annual global burden of disease was estimated to be US\$330 million, with a range between US $\$ 205$ and 459 million. This is the first detailed estimate of global costs due to vivax malaria and highlights the lack of cost data available to construct these estimates. Since regional extrapolations were required for the costs in many vivax-endemic areas, these estimates need to be interpreted with caution as the costs in countries where data are not available could be substantially different from the countries where data are available.

Chapter 6 also presents the results of two cost-benefit analyses of alternative global scenarios for case management. In the Highly effective radical cure scenario, G6PD screening was implemented and individuals who were G6PD normal were prescribed a supervised course of primaquine radical cure. Whilst implementing such a policy would increase the healthcare provider costs by nearly three-fold (from US $\$ 41$ million to US\$121 million) due to the assumed costs of supervised treatment, the potential benefits would result in a reduction in the overall costs by US $\$ 45$ million. Adherence to primaquine regimens remains a major concern as highlighted in Chapters 1 \& 4, so the Low adherence to radical cure scenario excluded the cost of supervising radical cure and decreased its effectiveness to $15 \%$. In this scenario fewer benefits were accrued due to averted cases, but overall modest cost savings were demonstrated at the healthcare provider level due to a lower investment in strategies to improve adherence. These 
scenarios are likely to be conservative, since effective radical cure will not only reduce relapses in individuals but also prevent the onward parasite transmission associated with those infections, and thus the subsequent case-load. This potential reduction in transmission was not included in the current analysis. Although widespread use of a supervised 14 day regimen of primaquine may not be feasible in many locations, the potential long-term benefits of radical cure may be far greater than the short-term costs. While an US\$80 million increase in healthcare provider expenditure in the Highly effective radical cure scenario is a large sum of money, it represents just $3 \%$ of the US\$2.7 billion spent on malaria control and elimination in 2016 [4].

\subsection{Methodological approaches}

Whilst the majority of the findings presented were empirical, some methodological contributions were also provided throughout. Chapters $2 \& 3$ use a Discriminant Analysis of Principal Components to split households into meaningful SES categories through the maximisation of between group variability while minimising the within group variability. This enabled the analysis to capture the heterogeneity in ownership that would not have been seen using the standard principal component analysis. Re-settled Papuans had been provided with house and land by the local government resulting in many of the poorer households having high levels of house and land ownership but little other disposable income. Unlike the principal components analysis, the results from the Discriminant Analysis of Principal Components were able to explain almost all of the differences between groups using only two discriminant functions.

Due to the heterogeneity in the epidemiology of vivax malaria and G6PD deficiency, Chapter 5 included the development of a customisable online model, which can be found at https://malaria.shinyapps.io/g6pd_screening/. For some model parameters with limited evidence, world experts in vivax malaria have a wide range of opinions that are difficult to reconcile into the single value needed for a decision model. While the sensitivity analysis addresses this to a certain extent, the online model provided a practical way to illustrate how their differing views would drive changes in 
the cost-effectiveness outcomes. It is hoped that this transparent analytical decision support tool will contribute to more rational and well-informed debates about how to treat vivax malaria.

Chapter 5 presented the results from standardised costing tools applied to collect household cost data across seven study sites in four countries and provider cost data in three countries. This is the first time this has been done for malaria, regardless of species. These costs are later expanded in Chapter 6 to present a first attempt to look at the global costs of vivax malaria treatment as well as the potential cost benefit of radical cure on the global cost burden. While this is rudimentary work, which should be seen as a thought experiment rather than a definitive costing study, Chapter 6 provides a framework for future research in this area by identifying knowledge gaps.

\subsection{Policy implications}

The generation of policy-relevant research has been a priority for this thesis. Meetings with the Asia Pacific Malaria Elimination Network (APMEN), PATH, MMV and GlaxoSmithKline have provided forums for ongoing interaction with policymakers and other stakeholders. During these meetings, results were presented, ideas for future work were generated and refined, and advice was exchanged on operational barriers to the roll out of G6PD tests and tafenoquine and future research needs. As a result, much of the information presented in this thesis relates to the costs and cost-effectiveness of radical cure, a pressing need in the field of vivax malaria. This thesis has demonstrated the potential cost-effectiveness of giving primaquine to patients after screening for G6PD deficiency to ensure safe radical cure. Chapter 4 included the cost of 14 days of primaquine therapy supervised by a healthcare worker in an attempt to match the effectiveness data from the clinical trial. While this is almost certainly an overestimate of the investment needed to ensure adherence, it was still cost-effective to screen for G6PD deficiency before radical cure. It is hoped that this will encourage both country policymakers and healthcare providers to pursue programs that give radical cure after G6PD screening. 
In order to prevent the adverse consequences of subsequent relapsing infections, novel public health strategies will be needed to educate both providers and populations about the benefits of taking a full course of primaquine. Considering the results in Chapter 4 \& 6, it is likely that these new strategies will be cost-effective, but it is difficult to do a cost-effectiveness analysis for an intervention that has not been designed. Interventions currently under consideration range from a few minutes of education for the patients receiving the medication to text message reminders to observed therapy by family members. These interventions will need to be adapted to the needs of the population where they are implemented. Regardless of method chosen, ensuring adherence to radical cure is important. This is highlighted by other studies in Timika, Indonesia: whilst Chapters $2 \& 3$ indicated that more patients were prescribed 14 days of primaquine after the policy change, the effectiveness of $P$. vivax radical cure remained suboptimal as evidenced by the observed degree of multiple recurrences following treatment with an unsupervised regimen [5]. A policy priority should be developing the tools needed to achieve effective radical cure: this will be an important milestone in the journey towards malaria elimination.

\subsection{Limitations}

The work presented in Chapters 2-6 presented a range of challenges in terms of data and methodology. The immediate challenge was to acquire cost data to use in cost-effectiveness models. As indicated in Chapter $\mathbf{1}$ and the review by White et al., limited previous studies have investigated the costs associated with $P$. vivax treatment. While the cost data presented in Chapter $\mathbf{2}$ is dated and the malaria epidemiology in that setting may limit its generalisability, it is valuable in that few other cost data sources exist, and it enabled the comparison of household costs for patients with $P$. vivax to those with $P$. falciparum. Chapter 5 presented new cost data from a range of countries. Together, these chapters greatly expand what is known about the costs of vivax malaria.

The methodology used to obtain costs, however, has not been consistent throughout 
the thesis in terms of inflation factors and conversion sources. In Chapters 2-3, the average exchange rate over one year was taken from www. OANDA.com [6], but in 2016 the website ceased providing this service for free. No other sources of annual conversion rates could be identified. As a result, Chapter $\mathbf{5}$ uses point estimates from mid-year (1 July) from www.XE.com [7]. While this methodology was applied consistently across all countries included in the analysis, these estimates from a single time point could bias the results if the date chosen saw a short-term spike or dip in the rate, which could be avoided if an average annual rate had been available.

Another alternative would have been to use purchasing power parity (PPP) rates to exchange local currencies into international dollars (I\$), which enables the comparison of currencies in terms of their ability to buy the same amount of goods and services. These are calculated using a large basket of goods and services, enabling a more reliable method of international economic comparisons than exchange rates. PPPs, however, have their own limitations. Generating PPPs for such a large basket of goods and services requires a huge amount of data, which means that calculations happen at infrequent intervals. The most recent survey was conducted in 2011, and PPPs since then have been based on estimates, potentially introducing measurement inaccuracies. Furthermore, the surveys do not cover all countries, which means that PPPs for missing countries must be imputed. Finally, while I\$ would enable a comparison of purchasing power across countries, the use of market exchange rates enables the aggregation of actual expenditures on vivax malaria across countries. Accordingly, Chapters 2-4 report US $\$$ since each uses costs from a single country, but $\mathrm{I} \$$ are reported in addition to US\$ in Chapter 5 to aid comparisons across countries. Since the aim of Chapter 6 was to estimate worldwide expenditures on vivax malaria treatment, costs are reported in US\$.

In Chapters 2 \& 3, the local currency was inflated to 2014 US\$ using the consumer price index (CPI) for Indonesia [8]. Chapter 4 also used CPIs to inflate costs. In Chapters 5 \& 6 the costs are inflated using GDP deflators [9]. While CPIs reflect changes in price level in a basket of goods and services for the average consumer, GDP 
deflators reflect price changes in the economy as a whole (i.e. a measurement of changes in the price level of GDP relative to real output). Since the GDP encompasses all aspects of the economy instead of those in a static basket of goods, it was deemed to be more appropriate for eliminating inflation over time. Considering the rapid rates of inflation over that time period, it is possible that the choice of CPIs in Chapters 2 \& 3 may have impacted the results. For example, a significant change in total costs over the entire fever period had been anticipated due to the availability of effective treatment. While it is not ideal to have presented such a range of methodologies and cost years in one document, these changes reflect a growth in understanding.

Another inconsistency related to the costs was the valuation of productivity losses. The survey described in Chapters $2 \& 3$ asked individuals to indicate their daily wage. The mean daily wage for all who reported one was calculated and multiplied by the number of days missed by the individual taking treatment in addition to any companions, caretakers and substitute labourers. For adults who work at home, this could mean an overestimate of the costs; however, it enabled the valuation of their time loss, which could be devastating to the households that depend on them. Children were not asked the number of days missed, which meant that their productivity costs were not included. This methodology was not possible in Chapter 5 since the trial surveys did not ask about wages. In order to account for productivity losses, GDP per capita per day was used to valuate each day lost to illness for both children and adults. Since vivax malaria disproportionately affects the poor and children, it is possible that this is an overestimate of the productivity losses. Given that the largest cost component in Chapter 5 was due to productivity losses, this assumption potentially had a large impact on the results in Chapters 5 \& 6 .

Chapters 2 \& 3 also presented challenges in terms of data management and interpretation. The data files were separated by survey sections and required merging and reshaping to prepare the data for analysis. In addition to not having a data dictionary, the variable and data labels were not always complete, so some data cleaning was required alongside careful study of the surveys. The time interval since collecting 
the survey data in 2005 meant that some of the individuals who had been involved were no longer contactable. Those individuals who were still available were relied upon to reconstruct some of the details of the study that were needed to understand the data and write those chapters.

The cost-effectiveness analysis in Chapter 4 also presented challenges in terms of data selection and presentation. Routine care could either be the chloroquine strategy or the primaquine strategy, which are two extremes of the spectrum as compared to the screening strategy. Separating these comparisons made the results more relevant to policy, but this made it difficult to choose what values would be the most conservative for the screening strategy. Since pregnant and lactating women are ineligible for primaquine and G6PD deficiency varies by gender, the results had to be further separated into males and females. This necessitated the presentation of four comparisons per setting. In order to explore the results in other settings without further increasing the number of comparisons presented in Chapter 4, an online tool was developed (https://malaria.shinyapps.io/g6pd_screening/). This model is freely available online to help policymakers and providers explore the potential cost-effectiveness of screening for G6PD deficiency before giving primaquine. The interactive model is also useful for investigating the impact of model parameters that have limited data available and radical differences in expert opinion (i.e. mortality due to primaquine-induced haemolyis if blood transfusion not given).

Due to logistical issues, the healthcare facility data presented in Chapter $\mathbf{5}$ were collected remotely. To address this challenge, tools were developed to enable local staff to collect cost data and applied consistently across sites. Cost data collected remotely could be more vulnerable to error and therefore less comparable across sites; however, every effort was made to standardise the data collection.

Lastly, many of the other limitations in Chapters 2-5 were related to the understanding of vivax malaria, which are mostly related to data availability as highlighted in Chapter 1. Particular issues include (1) the inability to distinguish a relapse from a new infection, (2) variations in G6PD severity and distribution, (3) variations in $P$. 
vivax relapse patterns and (4) the poorly understood relationship between primaquine adherence and effectiveness. What is known about these issues are brought together with the cost data and case estimates in Chapter 6. This chapter represents the first time that case estimates from the Malaria Atlas Project have been used to generate global cost burden estimates. The global cost burden estimates presented in Chapter 6 use the most comprehensive costs due to P. vivax to data, highlighting the areas where more research is needed.

\subsection{Future research directions}

In response to the research findings, policy implications and limitations, the following research priorities are proposed to address the knowledge gaps.

\subsubsection{Utilising the cost-effectiveness model}

Further data collection using economic questionnaires are currently being gathered as part of a large multi-centred primaquine clinical trial (IMPROV) that will be completed in March 2018 [10]. The cost-effectiveness model developed and presented in Chapter 4 will be parameterised to these additional data and will provide important information on patient characteristics and efficacy that will complement the unit cost data presented in Chapter 5. The efficacy data from this trial includes a one year follow up period for patients receiving seven day and 14 day primaquine treatment as well as patients who did not receive radical cure. This will enable exploration of the cost-effectiveness of further options for vivax malaria case management and how these vary by setting from a healthcare provider and societal perspective.

The cost-effectiveness model will also be adapted to incorporate data from recent clinical trials investigating the efficacy of tafenoquine, a new treatment that can achieve radical cure with administration of a single dose $[11,12,13]$. Tafenoquine is a long-acting drug, and may increase the risk of haemolysis. For this reason the manufacturer will recommend that it should only be prescribed once the patient has been confirmed to have 
$>70 \%$ of population mean G6PD activity. Novel and more stringent quantitative G6PD tests will be required to exclude individuals with intermediate deficiency, particularly in risk-averse environments where primaquine has not been widely used. Although this approach would exclude some patients who could probably receive primaquine safely, the increased confidence in the low risk of haemolysis is likely to improve provider compliance. Furthermore, tafenoquine's single dose regimen will circumvent the challenge of poor patient adherence to primaquine. Further analyses will be carried out to verify and quantify those potential benefits.

\subsubsection{Targeting vulnerable risk groups}

Vulnerable risk groups are highlighted throughout the thesis: from Chapter $\mathbf{2}$ where poor individuals were more likely to report fever in the past month to Chapters 4 \& 6 , which had to exclude pregnant and breastfeeding women from receiving primaquine in the analyses due to contraindications. Although pregnant women are typically given at least one test for malaria during antenatal care in endemic areas, research on alternative treatments for malaria in pregnancy have been largely neglected, despite increasing evidence of the negative impact of malaria episodes during pregnancy and on the subsequent development of the child $[14,15,16]$. Post-partum women are more likely to get $P$. vivax malaria and less likely to have $P$. falciparum episodes when compared to non-pregnant, non-post-partum women [17]. Hence, prevention and control of P. vivax could have significant health benefits in the most vulnerable populations and should be prioritised in the future. In the setting of the Thailand-Myanmar border described in Chapter 4, approximately $15 \%$ of patients have sub-microscopic infections [18]. Recent evidence indicates that primaquine is not excreted in the breast milk and so can be given to women who are breastfeeding [19], creating an opportunity for providing radical cure to women soon after delivery and before the next pregnancy. Further research is needed to investigate the cost-effectiveness of options for vulnerable risk groups. Some examples include using weekly prophylaxis or the postpartum delivery of primaquine in women diagnosed with vivax malaria during pregnancy. 


\subsubsection{Unified drug treatment}

Chloroquine-resistant P. vivax is on the rise [20], and in some areas where high-grade resistance is observed policymakers are switching to a universal policy of ACT for both P. falciparum and P. vivax malaria [21]. A unified drug treatment would be a pragmatic alternative to species-specific treatment, making diagnosis and treatment easier for the healthcare provider. The experience of such a strategy in Indonesia is described in Chapter 3; however, the risk of recurrent infection following ACT in some locations may be high. A study form Thailand found that $51 \%$ of individuals with P. falciparum treated with a short-lasting ACT and $20 \%$ of individuals treated with a long-lasting ACT came back with P. vivax within 63 days [22], which cannot be explained entirely by reinfection. It is hypothesised that these recurrent parasitaemias are due to the acute febrile illness of $P$. falciparum reactivating the dormant and hidden $P$. vivax hypnozoites. Indeed, the risk of returning with $P$. vivax infection following P. falciparum infection in a high relapse setting is higher than the risk of returning with $P$. vivax infection following blood stage treatment for $P$. vivax in settings with low frequency relapse [23].

In the context of malaria elimination, where the percentage of malaria cases due to P. vivax is increasing as efforts focus on P. falciparum, a unified treatment for malaria using ACT plus radical cure for all malaria cases could be a cost-effective alternative to treatment by species. Such a policy would ensure high efficacy against the asexual stages of the parasite even in areas where chloroquine resistance is emerging, as well as the potential benefits of preventing $P$. vivax relapses in patients with either acute falciparum or vivax malaria.

Microscopy and RDTs for both P. vivax and P. falciparum are known to misdiagnose the species of malaria, and this can result in the administration of inappropriate treatment. For example, a study in Ethiopia found that approximately $9 \%$ of $P$. falciparum microscopy confirmed cases tested RDT positive for $P$. vivax, whereas $1 \%$ of P. vivax microscopy cases tested RDT positive for P. falciparum only. Furthermore, $72 \%$ of $P$. falciparum mono-infected individuals and $11 \%$ of $P$. vivax mono-infected 
individuals according to microscopy tested as mixed infection with the RDT, suggesting that the most appropriate treatment would have been an ACT plus primaquine to treat both infections [24]. When microscopy is used, it can be difficult to differentiate between species of malaria at low parasitaemias. Under a unified drug policy, all cases will get drugs that are efficacious for both diseases, regardless of whether the RDT correctly identified the type of malaria the individual has, resulting in a decrease in the number of days individuals are infectious.

In Chapter 4, the increased costs due to drugs and G6PD testing was offset by the cost savings due to prevention of future episodes resulting from hypnozoites and an increase in the number of individuals receiving radical cure. Some settings may not have a high enough proportion of patients with falciparum malaria who have vivax hypnozoites to justify the increased cost of a unified treatment for malaria including G6PD screening and radical cure in those who test normal. In these settings, individuals with severe variants of G6PD-deficiency who test G6PD normal would be of particular concern as an incorrect prescription for radical cure for falciparum malaria could result in haemolysis. This risk may not be justifiable in patients who are at low risk of having a $P$. vivax relapse.

A model-based analysis would allow the circumstances under which a unified treatment might be cost-effective to be identified. The correct treatment and prevention of relapses will result in fewer episodes of clinical malaria and the associated decrease in DALYs. Extending this work to include onward transmission would enable the model to capture the additional benefit of primaquine in terms of reduced transmission due to relapses. The use of primaquine in patients with $P$. falciparum will increase the risk of haemolysis in G6PD deficient individuals who are prescribed primaquine, causing further costs and DALYs in those with significant haemolysis. This could be extended to standardising high-dose primaquine after G6PD screening in areas of co-endemicity. As demonstrated in Chapters 4 \& 6, provider and patient adherence influence primaquine effectiveness; however, once tafenoquine is available, the issues with patient adherence would be alleviated for those who are G6PD normal. Such integrated management 
could be key to ensuring that malaria elimination is achievable (Chapter $\mathbf{1}$ ).

\subsection{Conclusion}

This thesis contributes to an understanding of the costs and global economic burden of $P$. vivax and provides novel information on how to optimise $P$. vivax patient management, particularly the implementation of primaquine radical cure. Despite the limitations, this represents the most extensive estimation of the costs of vivax malaria to date, and the cost-effectiveness of its treatment. This information provides the foundation from which policymakers and healthcare providers can explore new drugs and strategies, including single dose tafenoquine and novel G6PD diagnostics, as they become available. Understanding the costs and cost-effectiveness of alternative options for vivax malaria treatment and control will be critical in the journey towards malaria elimination. 


\section{References for Chapter 7}

[1] Price RN, Douglas NM, Anstey NM. New developments in Plasmodium vivax malaria: severe disease and the rise of chloroquine resistance. Curr Opin Infect Dis. 2009;

[2] World Health Organization. Control and elimination of plasmodium vivax malaria: a technical brief; 2015.

[3] White MT, Yeung S, Patouillard E, Cibulskis R. Costs and Cost-Effectiveness of Plasmodium vivax Control. Am J Trop Med Hyg. 2016;95(6 Suppl):52-61. doi:10.4269/ajtmh.16-0182.

[4] World Health Organization. World malaria report 2017.; 2017.

[5] Douglas NM, Poespoprodjo JR, Patriani D, Malloy MJ, Kenangalem E, Sugiarto $\mathrm{P}$, et al. Unsupervised primaquine for the treatment of Plasmodium vivax malaria relapses in southern Papua: A hospital-based cohort study. PLoS Med. 2017;14(8):e1002379. doi:10.1371/journal.pmed.1002379.

[6] OANDA. Currency Converter: Historical Exchance Rates for 2005;Available from: http://www. oanda.com/currency/historical-rates/(Accessed: 3 March 2015).

[7] XE. Current and Historical Rate Tables. 2016;Available from: http: //www . xe.com/currencytables/?from=USD\&date=2016-07-01(Accessed: 8 September 2017).

[8] The World Bank. Inflation, consumer prices (annual \%). 2015;Available from: http://data.worldbank.org/indicator/FP.CPI.TOTL.ZG(Accessed: 4 August 2015).

[9] The World Bank. Inflation, GDP deflator (annual \%). 2016;Available from: www. data.worldbank.org/indicator/NY.GDP.DEFL.KD.ZG(Accessed: 08 September 2017). 
[10] IMPROV Study Group. Improving the radical cure of vivax malaria (IMPROV): a study protocol for a multicentre randomised, placebo-controlled comparison of short and long course primaquine regimens. BMC Infect Dis. 2015;15:558. doi:10.1186/s12879-015-1276-2.

[11] Llanos-Cuentas A, Lacerda MV, Rueangweerayut R, Krudsood S, Gupta SK, Kochar SK, et al. Tafenoquine plus chloroquine for the treatment and relapse prevention of Plasmodium vivax malaria (DETECTIVE): a multicentre, double-blind, randomised, phase $2 \mathrm{~b}$ dose-selection study. Lancet. 2014;383(9922):1049-58. doi:10.1016/s0140-6736(13)62568-4.

[12] GlaxoSmithKline. Ph 2B/3 Tafenoquine (TFQ) Study in Prevention of Vivax Relapse. National Library of Medicine (US). 2011;Available from: https://clinicaltrials.gov/ct2/show/NCT01376167 NLM Identifier: NCT 01376167(Accessed: 2017 August 17).

[13] GlaxoSmithKline. Study to Assess the Incidence of Hemolysis, Safety, and Efficacy of Tafenoquine (SB-252263, WR238605) Versus Primaquine in Subjects With Plasmodium Vivax Malaria. National Library of Medicine (US). 2014;Available from: https://clinicaltrials.gov/ct2/show/NCT02216123 NLM Identifier: NCT02216123(Accessed: 2017 August 17).

[14] Poespoprodjo JR, Fobia W, Kenangalem E, Lampah DA, Warikar N, Seal A, et al. Adverse pregnancy outcomes in an area where multidrug-resistant plasmodium vivax and Plasmodium falciparum infections are endemic. Clin Infect Dis. $2008 ; 46(9): 1374-81$.

[15] Imwong M, Boel ME, Pagornrat W, Pimanpanarak M, McGready R, Day NP, et al. The first Plasmodium vivax relapses of life are usually genetically homologous. J Infect Dis. 2012;205(4):680-3. doi:10.1093/infdis/jir806.

[16] Moore KA, Simpson JA, Wiladphaingern J, Min AM, Pimanpanarak M, Paw MK, et al. Influence of the number and timing of malaria episodes during pregnancy on 
prematurity and small-for-gestational-age in an area of low transmission. BMC Med. 2017;15(1):117. doi:10.1186/s12916-017-0877-6.

[17] Boel ME, Rijken MJ, Leenstra T, Phyo AP, Pimanpanarak M, Keereecharoen NL, et al. Malaria in the post-partum period; a prospective cohort study. PLoS One. 2013;8(3):e57890. doi:10.1371/journal.pone.0057890.

[18] Imwong M, Nguyen TN, Tripura R, Peto TJ, Lee SJ, Lwin KM, et al. The epidemiology of subclinical malaria infections in South-East Asia: findings from cross-sectional surveys in Thailand-Myanmar border areas, Cambodia, and Vietnam. Malar J. 2015;14:381. doi:10.1186/s12936-015-0906-x.

[19] Gilder ME, Hanpithakphong W, Hoglund RM, Tarning J, Win HH, Hilda N, et al. Primaquine Pharmacokinetics in Lactating Women and Breastfed Infant Exposures. Clinical Infectious Diseases. 2018; p. ciy235-ciy235. doi:10.1093/cid/ciy235.

[20] Price RN, von Seidlein L, Valecha N, Nosten F, Baird JK, White NJ. Global extent of chloroquine-resistant Plasmodium vivax: a systematic review and meta-analysis. Lancet Infect Dis. 2014;14(10):982-91. doi:10.1016/s1473-3099(14)70855-2.

[21] Douglas NM, Anstey NM, Angus BJ, Nosten F, Price RN. Artemisinin combination therapy for vivax malaria. Lancet Infect Dis. 2010;10(6):405-16. doi:S1473-3099(10)70079-7 [pii] 10.1016/S1473-3099(10)70079-7.

[22] Douglas NM, Nosten F, Ashley EA, Phaiphun L, van Vugt M, Singhasivanon P, et al. Plasmodium vivax recurrence following falciparum and mixed species malaria: risk factors and effect of antimalarial kinetics. Clin Infect Dis. 2011;52(5):612-20. doi:ciq249 [pii] 10.1093/cid/ciq249.

[23] Battle KE, Karhunen MS, Bhatt S, Gething PW, Howes RE, Golding N, et al. Geographical variation in Plasmodium vivax relapse. Malar J. 2014;13:144. doi:10.1186/1475-2875-13-144. 
[24] Ashton RA, Kefyalew T, Tesfaye G, Counihan H, Yadeta D, Cundill B, et al. Performance of three multi-species rapid diagnostic tests for diagnosis of Plasmodium falciparum and Plasmodium vivax malaria in Oromia Regional State, Ethiopia. Malar J. 2010;9:297. doi:10.1186/1475-2875-9-297. 